\title{
Sandia Combustion Research
}

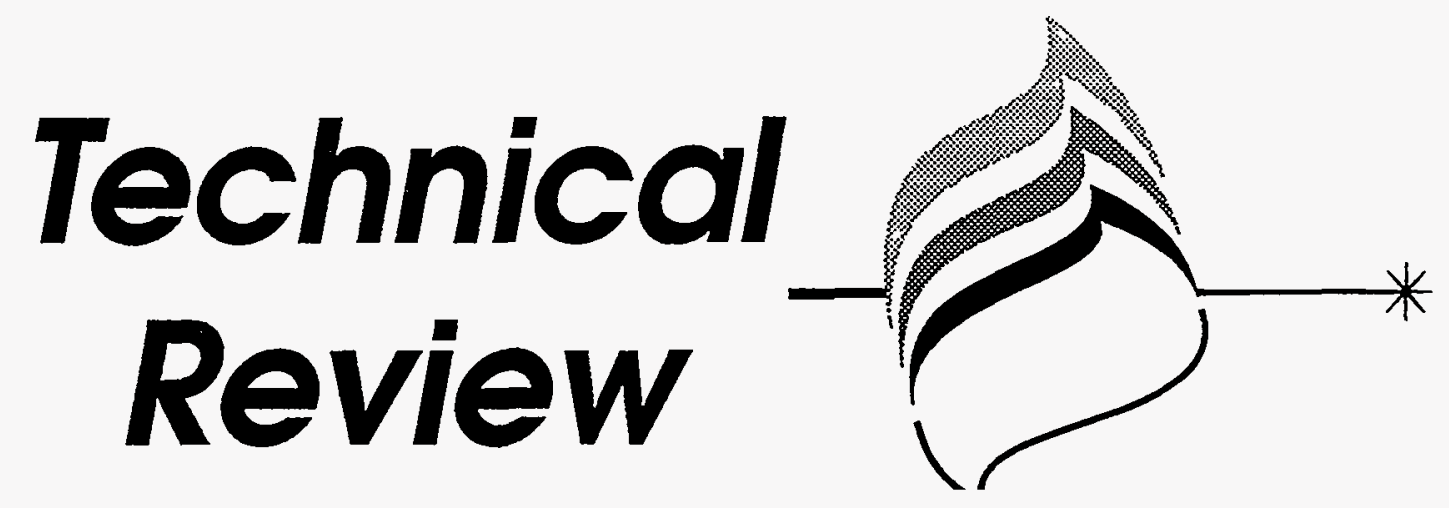

DISCLAIMER

This report was prepared as an account of work sponsored by an agency of the United States Government. Neither the United States Government nor any agency thereof, nor any of their employees, makes any warranty, express or implied, or assumes any legal liability or responsibility for the accuracy, completeness, or usefulness of any information, apparatus, product, or process disclosed, or represents that its use would not infringe privately owned rights. Reference herein to any specific commercial product, process, or service by trade name, trademark, manufacturer, or otherwise does not necessarily constitute or imply its endorsement, recommendation, or favoring by the United States Government or any agency thereof. The views and opinions of authors expressed herein do not necessarily state or reflect those of the United States Government or any agency thereof.

\section{5}




\section{Sandia Combustion Research Program Technical Review 1995}

To give comments or request more information, please contact

Dr. William J. McLean, Director Combustion Research and Technology Center Sandia National Laboratories

Livermore, California 94551-0969

(510) 294-2687 


\section{DISCLAIMER}

Portions of this document may be illegible in electronic image products. Images are produced from the best available original document. 


\section{CONTENTS}

Fifteen Years of Progress

W. J. McLean, Director

Visiting Researcher Program

4

J. C. McMichael

\section{Laser Based Diagnostics}

Introduction

L. A. Rahn

Planar Imaging of Differential Diffusion in Turbulent Flows.

P. H. Paul, K. A. Buch, N. T. Clemens, and G. B. Sartor

Probing Polyatomic Molecules using Infrared Degenerate Four-Wave Mixing

D. J. Rakestraw, R. L. Farrow, and G. J. Germann

Effects of Thermal Gratings and Collisional Quenching in UV Degenerate

Four-Wave Mixing

R. L. Farrow, P. M. Danehy, P. H. Paul, R. P. Lucht, and F. H. Blair

Full Characterization of Ultrashort Laser Pulses

R. Trebino, K. W. DeLong, D. N. Fittinghoff, and C. L. Ladera

Raman Lidar Profiling of Atmospheric Water Vapor

J. E. M. Goldsmith, S. E. Bisson, F. H. Blair, and M. G. Mitchell

The Development of a Laser-Based Imaging System for Long-Range Gas Plume

Visualization

T. J. Kulp and R. Kennedy

\section{Combustion Chemistry}

Introduction

F. P. Tully

Ion Imaging of Unimolecular and Bimolecular Reaction Products

D. W. Chandler, T. Kitsopoulos, R. McKay, A. Heck, A. Schiffman, M. Jaska, and R. N. Zare 
Femtosecond Laser Studies of Ultrafast Photochemical Processes

C. C. Hayden, D. W. Chandler, M. A. Gutzler, and D. R. Cyr

Laser-Induced Gratings in Molecular Spectroscopy and Chemical Dynamics.

30

E. A. Rohlfing, T. J. Butenhoff, J. R. Dunlop, J. D. Tobiason, S. Williams, and E. B. Bochenski

Kinetics of the $\mathrm{NH}_{2}+\mathrm{NO} \rightarrow$ Products Reaction

J. L. Durant, D. L. Yang, M. Wolf, H. S. Thiesemann, and M. Gutzler

Kinetics Investigations of the Reactions of $\mathrm{CH}$ [ $\left.\mathrm{X}^{2} \Pi\right]$ with $\mathrm{CO}$ and $\mathrm{O}_{2}$

C. A. Taatjes and R. T. Jennings

Thermal Decomposition of New Energetic Materials .

R. Behrens, Jr., L. M. Minier, D. M. Puckett, and S. Bulusu

The Oxidation of Allene in Flames.

J. A. Miller, J. V. Volponi, and J.-F. Pauwels

Theoretical Predictions of Fullerene Hydride Chemistry

C. M. Rohlfing, P. A. Cahill, and C. C. Henderson

Studies in Combustion Dynamics

M. L. Koszykowski, R. C. Armstrong, and J.-Y. Chen

\section{Reacting Flows}

Introduction

L. A. Rahn

Measurements of Nitric Oxide Formation in Turbulent Hydrogen Jet Flames

R. S. Barlow, C. D. Carter, and T. L. Prast

Temporal Evolution of Turbulence/Chemistry Interactions Studied in Nonpremixed Flames

R. W. Schefer, M. Namazian, and J. Kelly

Finite-Rate and Transient Effects Studied in Direct Numerical Simulations

of Turbulent Flames.

J. H. Chen, S. Mahalingam, and L. Vervisch 
Low Mach Number Reacting Flow Modeling

H. N. Najm

Premixed Flame-Speed Scaling in Low-Intensity Turbulence

A. R. Kerstein and W. T. Ashurst

Growth of a Turbulent Flame 56

W. T. Ashurst

The Deflagration of Porous Energetic Materials. 58

S. B. Margolis and F. A. Williams

\section{Combustion in Engines and Commercial Burners}

Introduction.

R. W. Carling, C. M. Hartwig, and J. 0. Keller

Quantitative Vapor-Fuel Imaging in a DI Diesel Engine using Planar Laser

Raleigh Scattering

C. Espey, J. E. Dec, and E. L. Porter

A Comparison of Crankcase and Blower Scavenging in a Fired Two-Stroke Cycle Engine

P. C. Miles, R. M. Green, P. O. Witze

Diesel Engine Combustion Modeling using the coherent Flame Model in Kiva-II. 66

B. C. Dillies, K. D. Marx, J. E. Dec, and C. Espey

Effects of Natural Gas Composition on Ignition Delay Under Diesel Conditions 68

J. D. Naber and D. L. Siebers

Characterization of the LDV Fringe Field 70

P. C. Miles and P. O. Witze

Research and Development on Advanced Gas-Fired Burners in the BERL 72

N. Fornaciari, R. Sanford, and L. Claytor

Statistical Structure of the Ideal Spray 74

C. F. Edwards and K. D. Marx 
SPCDE: A User-Friendly Computer Code for the Design of Practical

Pulse Combustion Systems

P. K. Barr, J. O. Keller, and J. A. Kezerle

NOx and CO Emissions from a Pulse Combustor Operating

in a Lean Premixed Mode.

J. O. Keller, T. T. Bramlette, P. K. Barr, and J. R. Alvarez

\section{Coal Combustion}

Introduction.

81

D. R. Hardesty

Heterogeneous Kinetics in the Late Stages of Coal Combustion

R. H. Hurt, K. A. Davis, and J. Ross

Materials Diagnostics Applied to Solid-Fuel Combustion Research

N. Y. C. Yang, T. J. Headley, R. H. Hurt, K. A. Davis, and M. J. Wornat

Combustion of Biomass-Derived Pyrolysis Dils.

K. A. Davis, M. J. Wornat, S. P. Huey, A. J. Salmi, B. G. Porter, J. Ross, G. C. Sclippa,

B. M. Jenkins, F. Winter, and L. L. Baxter

Formation of Ash Deposits in Biomass-Fired Boilers.

L. L. Baxter, G. C. Sclippa, A. J. Salmi, B. M. Jenkins, T. R. Miles,

T. R. Miles, Jr., R. W. Bryers, and L. L. Oden

FTIR Emission Spectroscopy Applied to In Situ, Real-Time, Surface

Chemistry Measurements.

L. L. Baxter, G. C. Sclippa, A. J. Salmi, G. H. Richards, D. K. Ottesen, and J. N. Harb

\section{Industrial Processing}

Introduction.

D. R. Hardesty

Understanding the Gas-Phase Decomposition of Ceramic Precursors

M. D. Allendorf, T. H. Osterheld, S. M. Ferko, and C. F. Melius

Gas-Phase Chemistry Involved in the Deposition of Carbon from Cyclohexane 96

T. H. Osterheld, M. D. Allendorf, and S. M. Ferko 
Modeling of Plasma-Based Semiconductor Manufacturing Equipment

S. R. Vosen, E. Meeks, J. W. Shon, R. S. Larson, and R. J. Kee

Hydrothermal Oxidation Kinetics of Methanol

100

S. F. Rice, R. G. Hanush, and R. R. Steeper

Destruction of Hazardous Munitions by Supercritical Water Oxidation

S. F. Rice, C. A. LaJuenesse, R. G. Hanush, and J. D. Aiken

Optical Monitoring of the Oxidation of Methane Under

Supercritical Water Conditions

R. R. Steeper, S. F. Rice, and J. D. Aiken

Optical Spectroscopy Applied to Process Control in Basic Oxygen Steelmaking

S. W. Allendorf, D. K. Ottesen, M. Bonin, K. Chen, H. A. Johnsen, D. Jung,

F. W. Kent, P. D. Stelts, and J. Wang

Miniature Optical Sensor Developed for Control of Basic Oxygen Steelmaking. 108

R. H. Hurt, M. P. Bonin, E.A.. Fuchs, P. D. Stelts, F. W. Kent, and A. J. Salmi

Publications 


\section{Sandia Combustion Research}

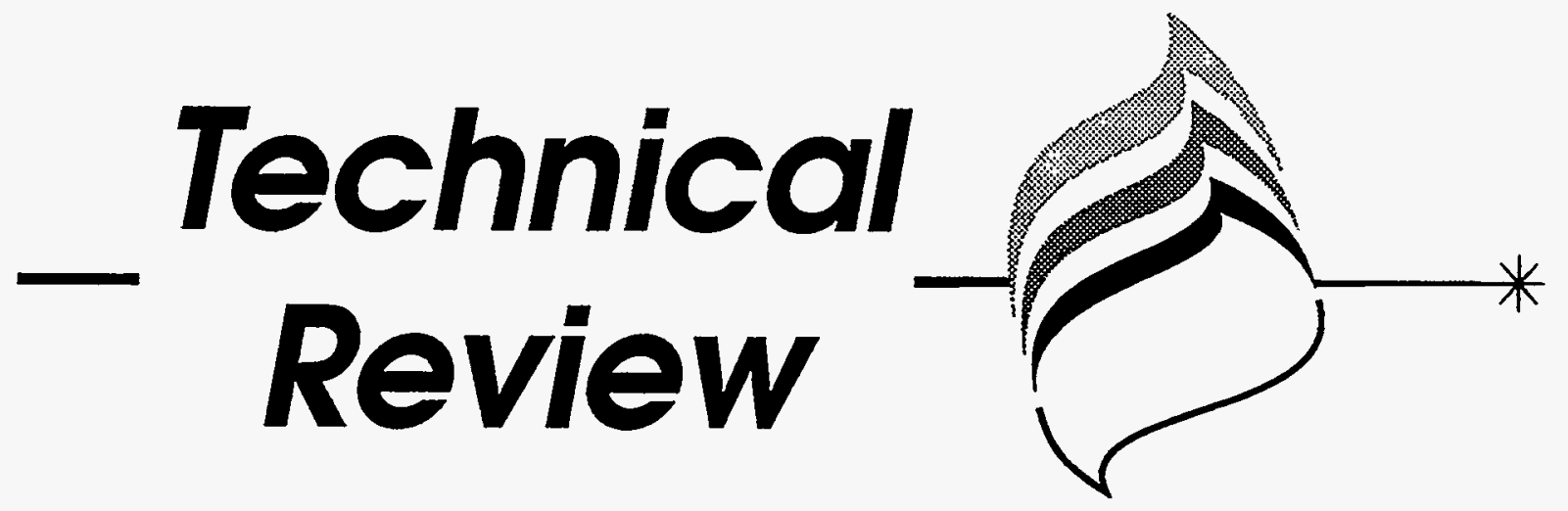

1995 


\section{Introduction}

\section{Fifteen Years of Prog̣ress}

\section{W. J. McLean, Director}

As this volume of the Combustion Research Facility's Technical Review goes to press, Sandia's CRF enters its fifteenth year as a Department of Energy Major User Facility. Combustion science and technology has progressed a great deal over these years, and no greater changes are observed than those related to the tools and approaches to combustion research. For example, it is now possible to observe and understand on a hundred femtosecond $\left(10^{-13} \mathrm{~s}\right)$ time scale the energy transfer processes associated with the molecular state-to-state transitions that, when integrated, constitute an elementary chemical reaction. In contrast, fifteen years ago we welcomed the increased accuracy of the measurements of the overall rates of these elementary reactions made possible then by the evolution of laser diagnostic methods. Similarly, we can now observe and understand spatially and cyclically resolved images of pollution formation inside practical internal combustion engines, whereas fifteen years ago we felt fortunate to observe high speed cine images of flame propagation in low compression ratio model engines bearing little resemblance to practical devices.

While the science and technology of combustion may have changed greatly in 15 years, the principal motivation for ongoing combustion research is still strongly focused on issues associated with energy and environment. The world-wide demand for ever cleaner and more efficient utilization of both fossil and renewable fuels requires that both fundamental and applied combustion processes be observed, interpreted, modeled, and understood at a level of detail not heretofore possible. The exploitation of advanced techniques to provide such details is particularly well illustrated in the CRF activities described in this review. For example, time resolved observations of molecular scale chemical processes and microscopic scale fluid processes are being made using state-of-the-art tunable lasers and planar imaging technologies. Also notable in this collection of articles is the fact that the techniques utilized for combustion studies are more and more frequently being applied to energy and environmentally related problems and processes that are well beyond regimes traditionally associated with combustion science and technology. 
In looking over the Technical Review, a major strength of the CRF's programs is evident: We have the ability to exploit state-of-the-art techniques for developing in-depth understanding of complex scientific issues and we apply such understanding to a broad range of industrially relevant problems. In short, we provide science-based-engineering solutions to energy and environmental problems of national importance.

The vitality of the CRF as a User Facility has reached an important milestone as we enter 1995 . With the Burner Engineering Research Laboratory (BERL) reaching full productivity, with new capabilities for imaging turbulent flames and measuring detailed chemical reaction dynamics coming on line, and with cooperative $R \& D$ agreements with major industries now in place, user demands have reached new highs. A following article provides more information on this trend, but suffice it to say here that demand for access to the CRF's people, programs, and capabilities continues to increase. We anticipate accommodating significantly more users when the additional laboratory capabilities and office space incorporated in our Phase II project are available some three years hence.

Other factors with potential effects on CRF scientific and technical programs are also prominent as we enter 1995. Federal budgets are under extreme pressure and the Department of Energy is carefully reviewing all its programs. The entire perspective of the federal role in research and development is being re-examined, and the utilization of the National Laboratories is being studied. While CRF activities will be appropriately scrutinized in these various evaluations, we feel that our solid record of scientific and technical productivity, service to facility users, and, most especially, our ability to translate the results of basic research programs into value added contributions to solving the nation's energy and environment problems will continue to serve us well during the next 15 years. 


\title{
Visiting Researcher Program
}

\author{
The Combustion Research Facility is designated by DOE as a "User Facility" and Sandia \\ National Laboratory offers the Facility's unique resources to academia and industry through \\ the sponsorship of a vigorous visitor program
}

\section{J. C. McMichael}

The Combustion Research Facility's (CRF) programs have, over the years, been closely aligned with industry. Further, the mission of the CRF includes an active involvement with the academia in studies relating to combustion Thus, Sandia pursues a robust visitor program that enables faculty and students from universities, and researchers from industry and other national laboratories to avail themselves of the unique resources of the facility.

The Cooperative Research and Development Agreements (CRADAs) between Sandia and the academic/industry combustion communities have established working and cost-shared funding arrangements that can result in on-site collaboration to commercialize láboratory technologies. In addition, Sandia directly invites researchers to the CRF to participate in collaborative research that is of benefit to both Sandia and the visitor.

Presently in place with industry is the Refinery Air Toxics CRADA where SNL has teamed with Texaco, Chevron, Amoco, Mobil, Southern Cal Gas and GRI. Also approved by DOE is the USCAR Low Emission Partnership with GM Research Lab and Cummins to develop new approaches to automotive combustion engines for control of NOx emissions. In addition the BERL CRADAs is in being finalized to more closely link industry with the research evolving in the Burner Engineering Research Laboratory

Research support provided to visiting researchers can include technician and engineering staff devoted to a given project, computer time, shop work, office space and housing. Visitors generally provide their own salary, travel and living expenses, augmented by project or Basic Energy Science funds as agreed to by the various project managers. Visitors agree that the results of their research will be made available in the open literature.

The CRF has hosted over 10,000 technical visitors over the last fourteen years. Many of the visits last only one or two days for discussions of specific combustion related problems. However, approximately seventy visitors each year stay for a week or more, often as long as a year or two. These long term assignees may be involved in CRADA activities or other research that involves close interaction with the CRF staff. Figure 1 shows the total number of visitors since 1985, while Figure 2 provides a summary of the number of long term visitors over the last ten years. Foreign visitors represented $15 \%$ of the long term visitors in 1994.

Included in Figure 2 are the Sandia Postdoctoral Program participants at the CRF. Each year the CRF draws upon physicists, chemists, mathematicians and engineers for one to two year appointments as Post Doctoral Associates who wish to work in the field of combustion research. At the completion of their appointments, they are generally move on to industry and universities providing yet another way to transfer CRF technology to the combustion community.

Visiting researchers are generally invited to the CRF by their professional peers within Sandia. Those interested in participating in the Visiting Researcher Program should contact the appropriate manager or staff member. For further information regarding the administration of the program, please contact the author. 


\begin{tabular}{|lll|}
\hline$\square$ University $\quad \square$ Industry & $\square$ Government \\
\hline
\end{tabular}

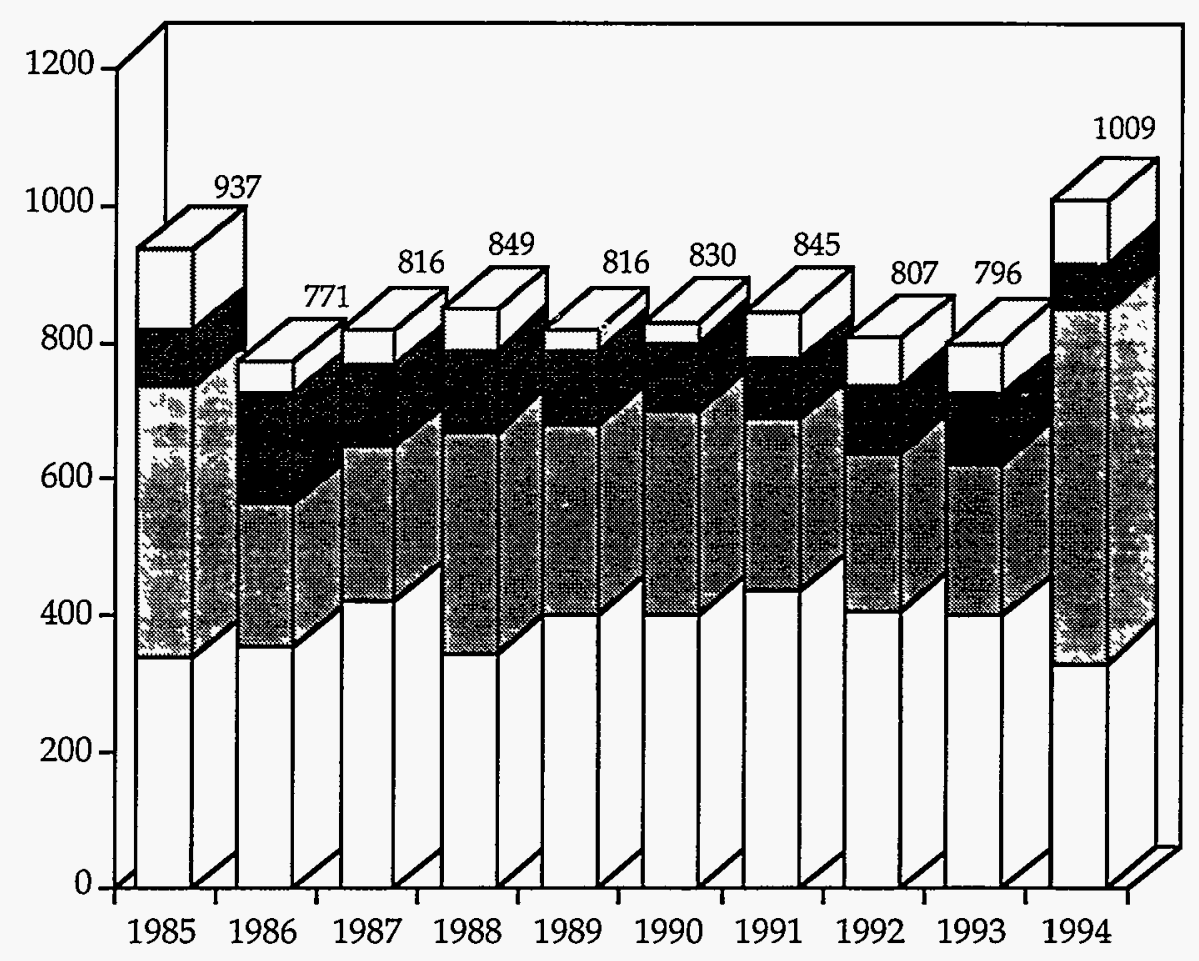

Figure 1. Total visitors to the CRF since 1985

\begin{tabular}{|ll|}
\hline$\square$ Postdoctorals & 固 University \\
$\square$ Government & $\begin{array}{l}\square \text { Industry } \\
\text { Labs } \\
\text { Lational }\end{array}$ \\
\hline
\end{tabular}

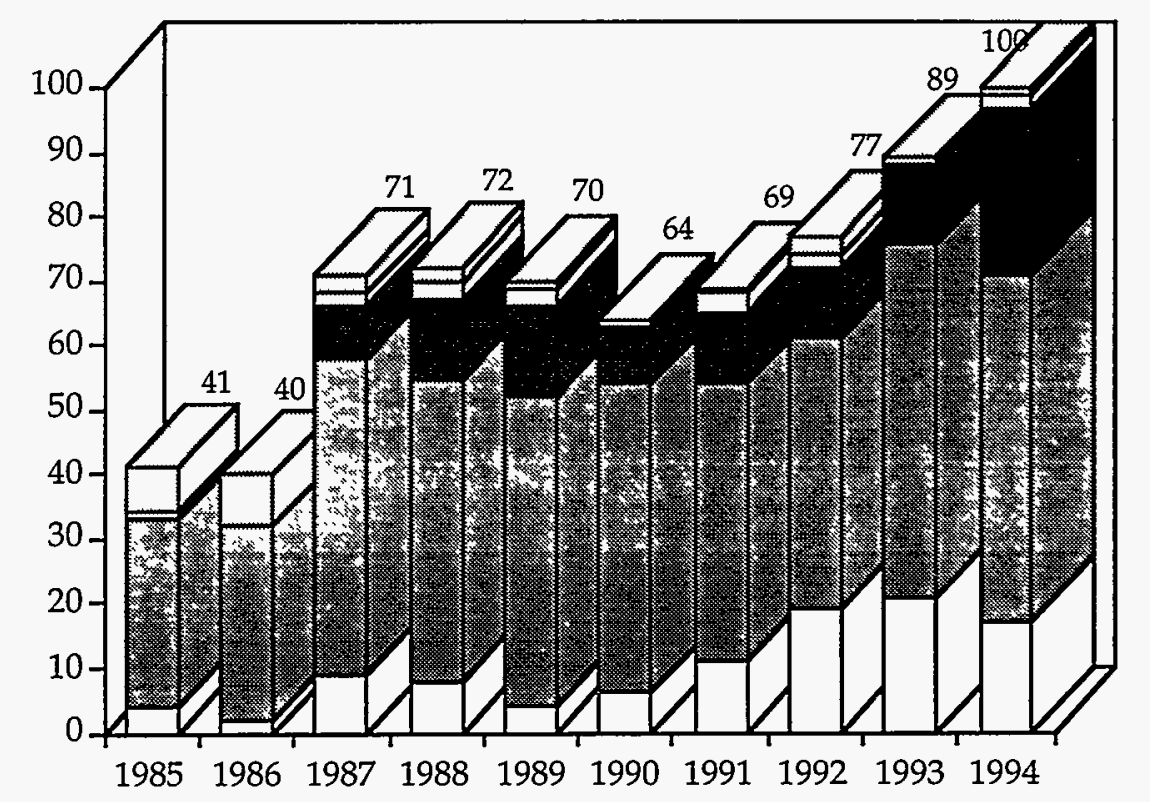

Figure 2. Long Term Visitors to the CRF since 1985 


\section{CRF VISITING RESEARCHERS}

January 1, 1994-December 31, 1994

\begin{tabular}{|c|c|c|c|}
\hline Name & Affiliation & Term of Stay & Collaborative Project \\
\hline \multicolumn{4}{|c|}{ Visiting Researchers from Universities } \\
\hline Adams, Peter & Cornell University & Sept. 1,94-Dec. 31,94 & $\begin{array}{l}\text { Behavior of inorganic } \\
\text { material during combustion }\end{array}$ \\
\hline Anderson, Don & Las Positas College & Mar. 28,94-Apr.15,94 & BERL Tests \\
\hline Bernath, Peter & Univ. of Waterloo & June 30,94 -July 29,94 & $\begin{array}{l}\text { New diagnostics for } \\
\text { inorganic material }\end{array}$ \\
\hline Buch, Ken & Univ. of Michigan & Feb.22,93-March 1,95 & Turbulent reacting flows \\
\hline Bui-Pham, Mary & UC San Diego & Oct. $19,92-S e p t .30,94$ & Flame ignition and extinction \\
\hline Card, John & UC San Diego & Nov. 2, 92-Nov. 1, 94 & Turbulent flame modeling \\
\hline Caton, Jerry & Texas A \& M Univ. & Sept. 1,93-May 30,94 & Diesel sooting studies \\
\hline Cattolica, Bob & UC San Diego & Jan. 1,94-June 1,94 & $\begin{array}{l}\text { Combustion diagnostic } \\
\text { development }\end{array}$ \\
\hline Chen, J.Y. & UC Berkeley & Jan 1,94-Dec. 31,94 & TDF Modeling \\
\hline Clemens, Noel & University of Texas & April 1,94-May 15,94 & TDF Studies \\
\hline Cyr, Douglas & UC Berkeley & $\operatorname{Jan} 3,94-\operatorname{Jan} 2,96$ & Femto 2nd laser experiment \\
\hline Dadoo, Rajeev & Stanford University & Aug. 1,93-July 30,96 & Advanced chemical detection \\
\hline Davis, Kevin & Princeton University & Oct. 1,92-Feb. 3,95 & $\begin{array}{l}\text { Combustion of bio-mass } \\
\text { derived pyrolysis oils }\end{array}$ \\
\hline Davis, Jeffrey & Cornell University & Sept. 1, 94-Dec. 31,94 & $\begin{array}{l}\text { Combustion of bio-mass } \\
\text { derived pyrolysis oils }\end{array}$ \\
\hline De Beers, Esther & UC Berkeley & July 15,93 -July 1,95 & Molecular imaging studies \\
\hline DeLong, Ken & Univ. of Arizona & Sept. 8,92-Aug. 31,94 & Ultra-fast phenomena \\
\hline Dibble, Bob & UC Berkeley & Jan. 1,94-Dec. 31,94 & TDF Studies \\
\hline Dunlop, James & Ohio State Univ. & July $13,92-J u l y ~ 1,94$ & Chemical kinetics research \\
\hline Edgar, James & Kansas State Univ. & Sept. 1,94-May 31,95 & Study silicon carbide \\
\hline Ehrhorn, Tina & Livermore High & May 23,94-Sept. 15,94 & Administrative Assistant \\
\hline Eppink, Andre & Univ of Nijmegen & April 4,94-June 30,94 & $\begin{array}{l}\text { Bio-molecular imaging } \\
\text { experiments }\end{array}$ \\
\hline Espey, Christoph & Penn State Univ. & Oct. 15,90-Dec. 30,94 & $\begin{array}{l}\text { Diesel engine combustion } \\
\text { processes }\end{array}$ \\
\hline Fischer, Ken & Univ. of Michigan & June 1,94-May 30,96 & Remote sensing \\
\hline Fittinghoff, David & UC Davis & Nov. 22,93-Dec. 1,95 & Laser pulse measurements \\
\hline Friedman-Hill, Ernest & MIT & Aug. 3,92-Sept. 1,94 & DFWM studies \\
\hline Garman, John & UC Irvine & Jan. 13, 94-Sept. 30,94 & $\begin{array}{l}\text { Laser diagnostics for } \\
\text { flame chemistry }\end{array}$ \\
\hline Germann, Geoff & UC Irvine & Nov. $1,92-$-Oct. 31,94 & DFWM Processes \\
\hline Goix, Philippe & Univ. San Francisco & June 6,94-June 30,96 & Refinery toxic emissions \\
\hline Gran, Inge & Norwegian TH & Sept. 1,94-Feb. 28,95 & Turbulent flame modeling \\
\hline Heck, Albert & Stanford University & Sept. $30,94-S e p t .30,95$ & Molecular imaging studies \\
\hline Jenkins, Bryan & UC Davis & Oct. 1,93-Sept. 30,94 & Biomass combustion probs \\
\hline Jung, Dan & Cornell University & May 31,94-Aug. 30,94 & Optical sensors \\
\hline Karlegard, Asa & Lund University & Aug. 15,94-Aug. 15,95 & SCWO experiment \\
\hline Kollmann, Wolfgang & UC Davis & Jan. 1,94-Dec. 31,94 & $\begin{array}{l}\text { Modeling turbulent reacting } \\
\text { flows }\end{array}$ \\
\hline Ladera, Luis & Simon Bolivar Univ. & Jan. 12,94-Dec. 31,94 & Laser pulse measurements \\
\hline Leinau, Jeff & UC Davis & Jan. 17,94-Aug. 5,94 & Reacting flow simulation \\
\hline Lucht, Bob & Univ. of Illinois & Dec. 15,92 -Dec. 31,94 & Reacting flow studies \\
\hline
\end{tabular}




\begin{tabular}{|c|c|c|c|}
\hline Name & Affiliation & Term of Stay & Collaborative Project \\
\hline \multicolumn{4}{|c|}{ Universities (continued) } \\
\hline Magdaleno, Alfonso & Phoenix High School & June $15,94-S e p t .15,94$ & ES \& $\mathrm{H}$ assistance \\
\hline Magnussen, Pia & Normegian TH & July 1,94-Sept. 30,94 & Turbulent flame modeling \\
\hline McKay, Ruth & Stanford University & June 1,92-Sept.30,94 & Bio-molecular imaging \\
\hline Miles, Paul & Cornell University & Sept. $15,92-$ Sept. 15,94 & Engine diagnostics \\
\hline Naber, Jeff & Univ. of Wisconsin & July 6,92-July 30,94 & Diesel engine processes \\
\hline Nandula, Sastri & Vanderbilt Univ. & May 23,94 -June 17,94 & TDF Studies \\
\hline Nguyen, Quang & UC Berkeley & Feb. 1,94-Dec. 31,94 & No formation in methane \\
\hline Nunes, Jon & San Diego State Univ. & Feb. 1,94-Dec. 31,94 & Optical isomers \\
\hline Osterheld, Tom & Stanford University & Aug. 31,92-Aug.30,94 & Materials synthesis \\
\hline Pitz, Bob & Vanderbilt Univ. & May 23,94 -June 17,94 & TDF Studies \\
\hline Ram, Ram & Univ. of Arizona & Mar. 1,94-May 31,94 & $\begin{array}{l}\text { FTIR characterization of } \\
\text { inorganic vapor }\end{array}$ \\
\hline Riedel, Uwe & Univ. of Stuttgart & Mar. 1,94-Feb. 28,95 & Chemical vapor disposition \\
\hline Roglingen, Judy & Las Positas & Dec. 10,93 -Sept. 30,94 & Advanced chemical detection \\
\hline Rosa, Daniel & Arrowhead H.S. & Aug. 8,94-Aug. 19,94 & $\begin{array}{l}\text { Modify software for flame } \\
\text { experiment }\end{array}$ \\
\hline Saari, Peeter & Estonian Academy & Oct. $11,94-$ Oct. 24,94 & Ultrashort pulse diagnostics \\
\hline Schiffman, Aram & Univ. of Colorado & Sept. 23,93-Aug. 30,95 & Reaction product imaging \\
\hline Sick, Voelker & Univ. of Heidelberg & July 30,94-July 30,95 & DFWM Experiments \\
\hline Sinquefield, Scott & Oregon State & Aug. 8,94-July 31,95 & $\begin{array}{l}\text { Low grade fuels, behavior } \\
\text { of ash }\end{array}$ \\
\hline Steuerwald, Stefan & Univ of Kaiserslauter & Oct. $15,94-N o v .12,94$ & Ion imaging experiments \\
\hline Thiesemann, Holger & Max Plank Institute & Oct. 1,94 -Oct. 31,95 & Gas phase chemical kinetics \\
\hline Tobiason, Joe & Univ. of Wisconsin & Sept. $13,93-$ Sept. 15,95 & Laser-induced spectroscopy \\
\hline Tong, Charles & Hong Kong University & July 11,94-Aug. 5,94 & Parallel equation solver \\
\hline Wang, Jason & Cornell University & Sept. 1,94-Dec. 31,94 & \\
\hline Warnatz, Jurgen & Univ. of Stuttgart & July 15,94 -Oct. 15,94 & $\begin{array}{l}\text { Combustion chemistry } \\
\text { calculations }\end{array}$ \\
\hline Williams, Skip & Stanford University & April 1,93-Feb. 1,94 & DFWM studies \\
\hline Wilson, Steve & Univ. of Bristol & Aug. 20,94-Sept. 30,94 & $\begin{array}{l}\text { Biomolecular imaging } \\
\text { experiment }\end{array}$ \\
\hline Wilson, Andrew & UC Berkeley & May 24,93-Sept. 30,94 & $\begin{array}{l}\text { Particulate heat transfer } \\
\text { modeling }\end{array}$ \\
\hline Yan, Chao & Stanford University & Jan. 3,94-Dec. 31,95 & Advanced chemical detection \\
\hline Yang, David & Emory University & May 17,93-June 1,95 & Gas phase chemical kinetics \\
\hline Zhao, Hui & Stanford University & June 13,94 -June 30,96 & Advanced chemical detection \\
\hline
\end{tabular}




\begin{tabular}{|c|c|c|c|}
\hline Name & Affiliation & Term of Stay & Collaborative Project \\
\hline \multicolumn{4}{|c|}{ Visiting Researchers from Industry } \\
\hline Benson, Charles & A D Little & Nov. 1,93-Mar. 1,94 & BERL tests \\
\hline Boulianne, Robert & Canada EER & Sept. 12,94-Dec. 31,96 & $\begin{array}{l}\text { Conducting emission } \\
\text { measurements }\end{array}$ \\
\hline Campbell, Robert & EER Corp. & Sept. $12,94-$ Dec. 31,96 & $\begin{array}{l}\text { Conducting emission } \\
\text { measurements }\end{array}$ \\
\hline Carpenter, Richard & Hauck Mfg. Co. & Nov. 1,93-Mar. 1,94 & BERL tests \\
\hline Chan, Michael Acurex & & May 9,94-Aug. 31,94 & Burner evaluation \\
\hline Chu, Edward & Acurex & May 9,94-Aug. 31, 94 & Burner evaluation \\
\hline Claytor, Lloyd & EER Corp. & July 1,92-Dec. 30,94 & BERL support \\
\hline Cole, Gerald & EER Corp. & July 1,92-Dec. 31,94 & BERL support \\
\hline Elashi, Walied & EER Corp. & Sept. $11,94-$-Dec. 31,96 & Petroleum envir. research \\
\hline England, Glenn & EER Corp. & Sept. $11,94-$-Dec. 31,96 & Petroleum envir. research \\
\hline Fukuda, Mike & EER Corp. & Sept. 11,94 -Dec. 31,96 & Petroleum envir. research \\
\hline Fullerton, William & Canada EER & Sept. $11,94-$ Dec. 31,96 & $\begin{array}{l}\text { Conducting emission } \\
\text { measurements }\end{array}$ \\
\hline Glauser, Bill & Autodesk, Inc. & Mar. 16,92-Mar. 30,94 & $\begin{array}{l}\text { Theoretical chemistry, } \\
\text { molecular dynamics }\end{array}$ \\
\hline Huey, Sidney & Acurex & June 6,94-June 30,96 & Biomass pyrolysis \\
\hline Ladd, Eric & EER Corp. & Sept. $11,94-$-Dec. 31,96 & Petroleum envir. research \\
\hline Manke, Jeff & EER Corp. & July 1,92-Dec. 30,94 & BERL support \\
\hline McAllum, Peter & EER Corp. & July 18,94 -July 18,95 & BERL engineer \\
\hline McGrath, Thomas & EER Corp. & Sept. 11,94-Dec.31,96 & Petroleum envir. research \\
\hline Payne, Roy & EER Corp. & Apr. $20,92-A p r .20,94$ & BERL support \\
\hline Perry, Bob & Technor & Apr. $30,94-A p r .30,95$ & N20 reduction modeling \\
\hline Sanford, Robert & EER Corp. & July 1,92-Dec. 30,94 & BERL support \\
\hline Steurewald, Stefan & Kurt Schumacher & Oct. $17,94-$ Nov. 10,94 & Ion imaging experiments \\
\hline Thijssen, Johanne & AD Little & Nov. 1,93-Mar. 1,94 & BERL tests \\
\hline Voelkel, Dirk & MPI/Gottingen & Apr. 5,94-Mar. 31,95 & DFWM experiments \\
\hline Wolf, Matthias & MPI/Gottingen & Apr. 1,93-Mar. 25,94 & Gas phase chemical kinetics \\
\hline Wong, Derek & Acurex & May 9,94-Aug. 31,94 & Burner evaluation \\
\hline Zimperman, Robert & EER Corp. & Sept. 11,94-Dec. 31,96 & Petroleum envir. research \\
\hline
\end{tabular}

Visiting Researchers from Government and National Laboratories

\begin{tabular}{|c|c|c|c|}
\hline Bench, Graham & LLNL & July $19,93-J u l y ~ 18,95$ & Ion beam analysis \\
\hline Bulusu, Surya & DOA/Picatinny & Feb. 28,94-Mar. 4,94 & $\begin{array}{l}\text { Energetic materials } \\
\text { thermal decomposition }\end{array}$ \\
\hline Carter, Cam & WPAFB & Apr. 11,94-May 1,94 & Turbulent flame exper. \\
\hline Land, Terry & LLNL & Sept. 28,92-Sept.30,95 & $\begin{array}{l}\text { Energetic materials } \\
\text { thermal decomposition }\end{array}$ \\
\hline
\end{tabular}




\section{Laser-Based Diagnostics}

\section{Introduction}

An important thrust at the Combustion Research Facility (CRF) is the research and development of laser-based diagnostic methods. These methods enable nonintrusive quantitative measurements of species concentration, temperature, velocity flow fields, and particulates with high spatial and temporal resolution in combustion and environmental applications. Laser-based optical diagnostics have revolutionized combustion research over the past two decades, and expertise in their development and application is one of the cornerstones on which the Combustion Research Facility is founded.

Diagnostics research at the CRF includes three important components: the development of innovative approaches and techniques for optical diagnostics, research to understand limitations and provide a quantitative interpretation of these methods, and the development and transfer of measurement technology to applications. This work has benefited and often led the development of new techniques such as coherent anti-Stokes Raman spectroscopy, optogalvanic and multiphoton spectroscopies, resonant degenerate four-wave mixing, and thermal grating spectroscopy. Studies in collisional energy transfer, fluorescence quenching, high laser intensity effects, highresolution spectral lineshapes, and nonlinear optics have contributed significantly to our understanding of the limitations and quantitative interpretation of optical diagnostics. Advances in laser diagnostics require continued development in laser, optical and detector technologies as well. Our work has lead to new technology for injection seeding, optical isolation, ultra fast pulse characterization, and has spawned a new effort at the CRF in laser remote sensing. Many of the recent achievements in the areas mentioned above are represented in the following articles.

Sandia's efforts to develop advanced laser-based diagnostics are interdisciplinary and broadly based. The fundamental research in this area forms the core of the effort and is funded by the Chemical Sciences Division of the Office of Basic Energy Sciences, United States Department of Energy Work in this program is complemented by work in other basic and applied programs where diagnostic techniques are developed and applied to specific applications. 


\title{
Planar Imaging of Differential Diffusion in Turbulent Flows
}

\author{
Advanced planar imaging techniques have been used to study differential diffusion \\ in turbulent flows.
}

\section{P. H. Paul, K. A. Buch, N. T. Clemens, ${ }^{1}$ and G. B. Sartor}

Planar imaging techniques provide the experimentalist with a powerful means to study complex turbulent flows. However, the measured signals most often have a complex dependence on temperature, the molecular constituents, pressure, and even velocity. Quantitative measurements can be made within a single image when flow conditions can be arranged in a way that there is an isolated dependence on a single scalar variable. In more complex flows ratiometric processing of fluorescence images can be used to isolate a dependence on a single flow variable. We describe a multiple camera technique which allows for the imaging of two scalar fields which are characterized by different rates of molecular diffusion.

In the present study we employ a jet of propane issuing into a coflow of helium seeded with approximately $300 \mathrm{ppm}$ of NO. To first order this flow can be taken as two independent binary diffusion systems, NOpropane and He-propane, where the diffusivity of helium into propane is approximately 3.2 times that of $\mathrm{NO}$ into propane. Simultaneous planar images of the NO and propane fields allow for the determination of the scalar dissipation of each component as well as a quantitative measure of differential diffusion.

The propane field is imaged via planar Rayleigh scattering using a doubled YAG laser. The Rayleigh scattering cross-section for propane is nominally 850 times that for helium. Planar laser-induced fluorescence is employed to image the NO by exciting the $Q_{1}(10)$ transition in the NO $\gamma$-band with an excimer-pumped dye laser doubled in BBO.

${ }^{1}$ Dept. of Aerospace and Engineering Mechanics, University of Texas at Austin, Austin TX 78712
Propane and helium do not collisionally quench NO providing excellent signal levels due to a near unit fluorescence efficiency. Spectral filtering and temporal gating are used to discriminate the two images which are acquired on separate intensified CCD array cameras. The experiment was arranged with counter-propagating laser sheets and the laser pulses were separated by a delay of approximately one microsecond.

Multiple camera measurements are quite sensitive to the corrections required for laser sheet intensity variations and to the relative geometric match between the images. The data required to geometrically match the images was obtained by simultaneously recording with both cameras single-shot images of the particles normally in room air. These data were then used to develop a third-order image warping function to provide for final geometric matching during image post processing.

The image field-of-view was selected to include both free-streams of the axisymmetric mixing layer. Since the laser sheets were directed perpendicular to the mixing layer, the sheet intensity distributions could be taken directly from the free-stream (i.e. undisturbed fluid) signal profiles. These data were then used to provide the laser sheet profile corrections on a shot-by-shot basis. The free stream data was also used to normalize the images to provide images of mixture fraction, $\xi$. Since NO is present only in trace amounts, the mixture fraction of helium is given by $\xi_{\mathrm{He}}=1-\xi_{\text {propane }}$

Fig. 1 shows a PLIF image of NO and the corresponding image of the helium as derived from the propane image taken in an axisymmetric shear layer. These data are 

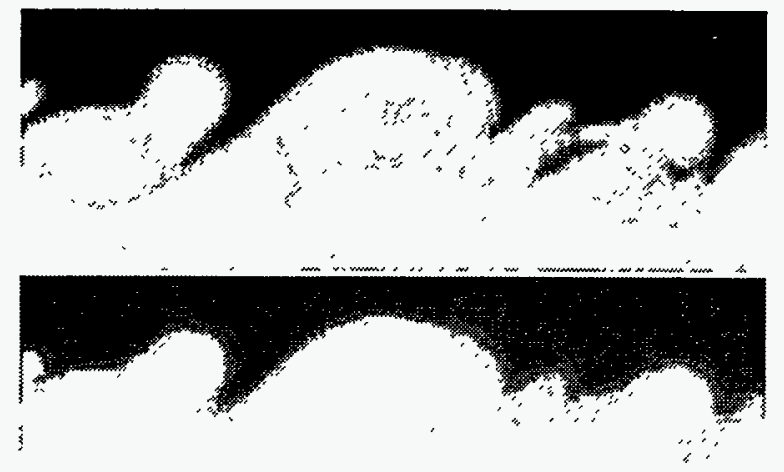

Figure 1. Mixture fraction of NO (upper) and He (lower) in an axisymmetric mixing layer. High speed fluid is on the top of the panels and flow is from left to right. The Reynolds number based on scalar thickness at midimage is 29,000 .

displayed as mixture fractions. The NO image shows much sharper gradients than the helium image to the extent that much of the structure in the vortex cores is gone in the helium image. In the absence of differential diffusion these two images would be identical.

The quantitative measure of differential diffusion that has been adopted is $z \equiv \xi_{\mathrm{NO}}-$ $\xi_{\mathrm{He}}$. Positive values of $z$ correspond to regions which are rich in NO whereas negative values indicate regions in which the helium has diffused into the propane ahead of the NO. In the absence of differential diffusion the entire $z$ field would be uniformly zero. In a shear layer the fluid is well stirred in the vortex cores creating a significant amount of interface and is thus highly subject to differential diffusion. In contrast the braid regions (between successive vortices) are under strong compressive strain and thus display a small but measurable effect.

Of wider interest than the $z$ variable is a measure of the differential diffusion as conditioned on the scalar dissipation which can be used to test turbulence and mixing models. The scalar dissipation of the differential diffusion, $\chi_{z} \equiv \nabla z \bullet \nabla z$, as derived from the data of Fig. 1, is given in Fig. 2. The data indicate that the production (or destruction) of the differential diffusion is largely localized to the layer interface and that it also closely follows the dissipation in the NO field.

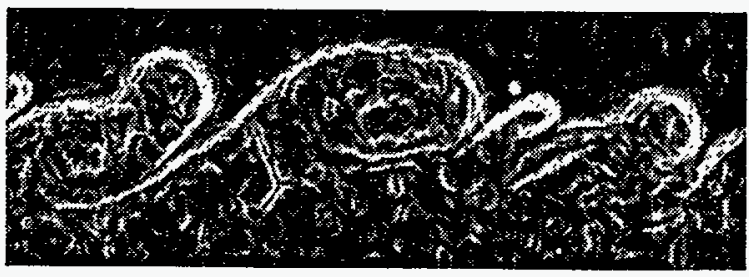

Figure 2. Scalar dissipation of the differential diffusion derived from the data of Fig. 1. The color table is continuous and logarithmic.

We have applied combined planar Rayleigh scattering and planar laser-induced fluorescence of NO to study mixing in a nonreacting axisymmetric shear layer. In order to use these data to derive higher order properties of the flow it was essential to apply both geometric image warp and laser sheet intensity corrections to the images. It must be expected that relative geometric errors between the cameras are the rule rather than the exception and that these errors will vary as a function of position across the image. We have described a technique that provides corresponding images of differential diffusion and scalar dissipation rate in a turbulent flow taken with flow stopping temporal resolution. These data can be used to develop statistical information on differential diffusion conditioned on scalar dissipation and are thus directly useful for testing general models of the effects of turbulence on molecular mixing.

This work was supported by the Division of Chemical Sciences, Office of Basic Energy Sciences, U.S. Department of Energy. 


\section{Probing Polyatomic Molecules using Infrared Degenerate Four-Wave Mixing}

The detection of polyatomic molecules presents a difficult but necessary task required for understanding dynamic chemical systems. Infrared degenerate four-wave mixing is expected to become a powerful tool for the accomplishment of this goal.

\section{J. Rakestraw, R. L. Farrow, and G. J. Germann}

In order to characterize and monitor a dynamic chemical system, such as a flame or reacting gas mixture, the chemical composition of the system must be determined in detail. Spectroscopic techniques employing laser light have proven to be extremely useful for the nonintrusive analysis of both transient and stable chemical species. Laser-induced fluorescence (LIF) is a technique widely used for detecting trace atomic and molecular species. The availability of commercial lasers operating in the visible and ultraviolet spectral regions has facilitated the wide spread application of LIF in this spectral region, but despite it's tremendous success, LIF is limited in it's application to a small set of polyatomic molecules that have low lying electronic states. The infrared spectral region, which probes rotational-vibrational transitions, is rich with information concerning almost all polyatomic molecular species; however the long upperstate lifetimes make infrared LIF impractical.

To date, the detection of molecules via rotational-vibrational transitions has been dominated by infrared absorption spectroscopy. Absorption spectroscopy allows the detection of a vast array of chemical species but it's applications are limited to cases where path integrated techniques are acceptable. In addition, absorption methods generally have reduced sensitivity compared to LIF since absorption is a differential measurement rather than a null background technique. Linear and non-linear Raman techniques can also be used to probe rotational-vibrational transitions and can provide high temporal and good spatial resolution. However, Raman techniques have relatively poor detection sensitivities and are restricted to the measurement of species whose concentrations are generally greater than $0.1 \%$.

We are currently investigating the use of degenerate four-wave mixing (DFWM) for the detection of polyatomic molecules via infrared active transitions. Through the use of a completely resonant process this nonlinear optical technique provides sensitive null background detection and permits high temporal and spatial resolution. We believe that this development will dramatically advance detection capabilities for a large range of polyatomic molecules, without introducing significant temporal and spatial averaging.

The experimental configuration used for the infrared DFWM experiments is similar to that reported previously for electronically enhanced DFWM [1]. The most significant difference is that the infrared laser light is generated by pumping an optical parametric oscillator with a single-longitudinal-mode Nd:YAG laser. A detailed description of the infrared experimental configuration can be found elsewhere [2].

We have investigated the spectroscopic application of DFWM for the measurements of methane, acetylene and nitrogen dioxide. A portion of the $Q$ branch of the methane $v_{3}$ band is shown in Fig. 1. This spectrum, which was acquired using low laser intensities in order to reduce saturation effects, demonstrates the high resolution and sensitivity achievable with infrared DFWM.

The nonlinear dependence of the DFWM interaction on laser intensity requires that we properly normalize the signals by the input powers. Under unsaturated conditions 


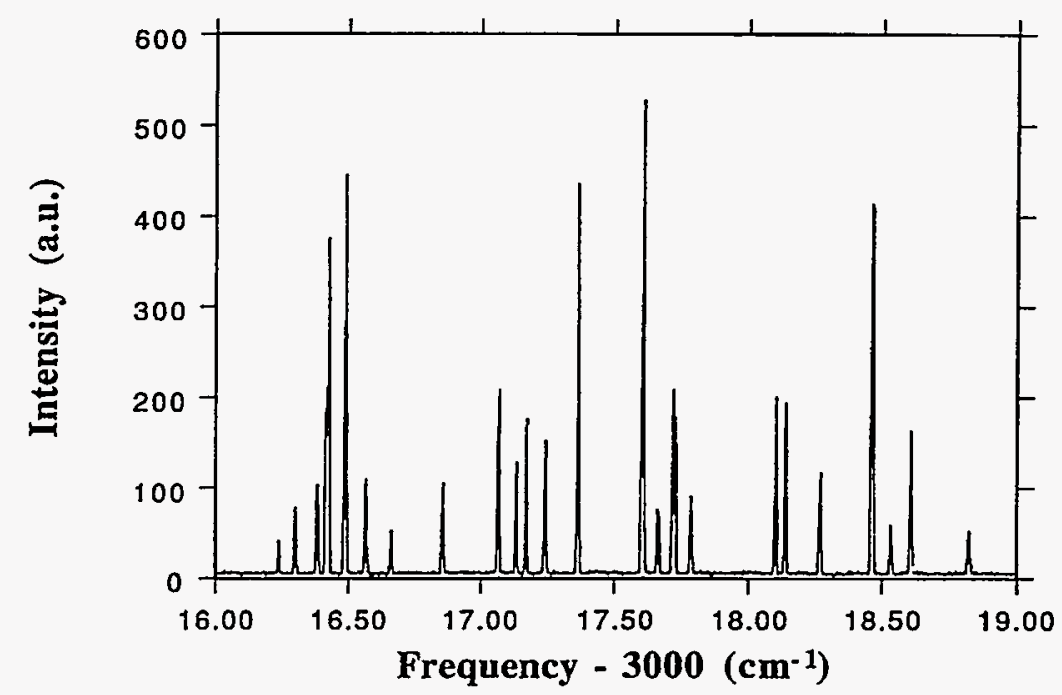

Figure 1. Infrared DFWM spectra of $\mathrm{CH}_{4}$ at $10 \mathrm{mTorr}$ total pressure. The observed transitions are the $Q(1)-Q(7)$ multiplets of the $v_{3}$ band. Pump and probe laser powers of $\sim 5 \mu \mathrm{J}$ were used which resulted in a small amount of saturation broadening.

normalization is satisfactorily accomplished by using $\mathrm{I}^{3}$. More generally the signal intensity can described by the stationary-absorber model developed by Abrams et al. [3] which includes saturation effects. Fig. 2 illustrates the good agreement between this model and the observed intensity dependence of the DFWM signal. In this case, the measured laser intensity range was well below the saturation intensity which resulted in a nearly $\mathrm{I}^{3}$ dependence.

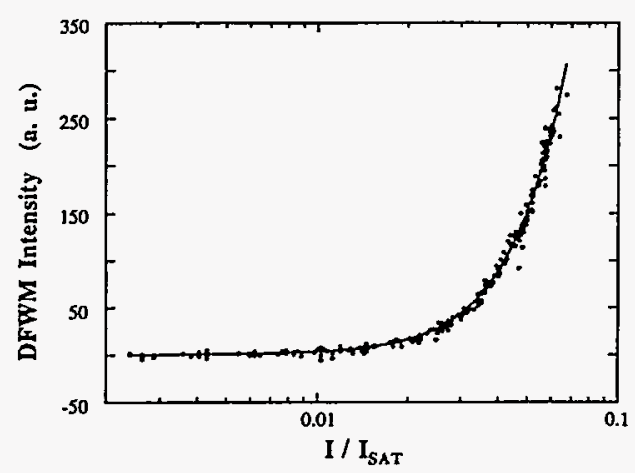

Figure 2. The observed power dependence of the infrared DFWM signal. The filled circles represent measurements from individual laser pulses and the solid line is the fit. The measurement was conducted on the $R(0)$ transition of the methane $v_{3}$ band with 800 Torr of nitrogen buffer gas.
Future infrared DFWM experiments will continue to focus on the detection of polyatomic molecules. We will begin to examine the ability of DFWM to probe complex gas mixtures exhibiting substantial spectral congestion in the mid-infrared. A major part of our current eifort is aimed at testing the ability of current theoretical models to predict experimental spectra by using DFWM simulation and fitting codes. In addition, we plan to exploit the use of infrared DFWM to make measurements of species profiles in low pressure flames.

This work was supported by the Division of Chemical Sciences, Office of Basic Energy Sciences, U.S. Department of Energy.

\section{References}

[1] R. L. Farrow and D. J. Rakestraw, Science, 257, 1894 (1992).

[2] G. J. Germann, A. McIlroy, T. Dreier, R. L. Farrow, and D. J. Rakestraw, Ber. Bunsenges. Phys. Chem., 97, 1630 (1993).

[3] R. L. Abrams, J. F. Lam, R. C. Lind, D. G. Steel, and P. F. Liao, "Phase Conjugation and High-Resolution Spectroscopy by Resonant Degenerate Four-Wave Mixing," in Optical Phase Conjugation, R. A. Fisher, ed., (Academic Press, New York, 1983), p. 211. 


\title{
Effects of Thermal Gratings and Collisional Quenching in UV Degenerate Four-Wave Mixing
}

\author{
The sensitivity of degenerate four-wave mixing to collisional quenching has been \\ characterized for UV excitation of nitric oxide. The thermal-grating scattering \\ mechanism has been isolated and studied.
}

\section{R. L. Farrow, P. M. Danehy1, P. H. Paul, R. P. Lucht², and F. H. Blair}

Resonant degenerate four-wave mixing (DFWM) has demonstrated considerable promise as sensitive diagnostic tool for atomic and molecular species[1]. DFWM generates a coherent, spectrally bright signal beam, providing excellent applicability in harsh combustion environments. The sensitivity of DFWM can approach that of laser-induced fluorescence (LIF), but, as an absorption rather then emission-based method, DFWM can also be used to detect non-fluorescing species. While LIF is a powerful and easily implemented technique, the dependence of LIF signal strengths on collisional electronic quenching rates requires knowledge of the chemical composition of buffer gases and relevant quenching cross sections. Early investigations suggested that DFWM spectra were inherently less sensitive to quench rate variations than were LIF spectra[2].

To further investigate this possibility, we used single-frequency laser radiation to perform DFWM intensity measurements on an isolated line in the $A-X$ transition of $\mathrm{NO}$ near $226 \mathrm{~nm}$. By using mixtures of $\mathrm{N}_{2}$ and $\mathrm{CO}_{2}$ buffer gases, we varied the collisional quenching rate over several orders of magnitude while maintaining a fixed total collisional dephasing rate. The varying quenching rate also had the effect of varying the conversion of excited-state population gratings into heat, resulting in the generation of thermal gratings. At total buffer-gas pressures near 100 Torr, the populationgrating mechanism dominated, while at higher pressures thermal-grating diffraction

\footnotetext{
${ }^{1}$ High Temperature Gasdynamics Laboratory, Stanford University, Stanford, CA 94305-303

'University of Illinois at Urbana/Champaign, 1206

W. Green St., MC-22, Urbana, IL 61801
}

became important, permitting detailed comparisons of both mechanisms.

The experimental setup used a phaseconjugate geometry and was similar to that described in Ref. 1. Measurements were performed on $\sim 30$ mTorr of NO in mixtures of $\mathrm{N}_{2}$ and $\mathrm{CO}_{2}$ contained in a static cell. DFWM and LIF signal intensities were simultaneously detected using photomultiplier tubes. In all measurements, we probed a well-isolated satellite line, $O_{12}(2)$, of the $A^{2} \Sigma^{+} \quad\left(v^{\prime}=0\right) \leftarrow$ $X^{2} \Pi\left(v^{\prime \prime}=0\right)$ band. LIF was detected through 10-nm bandpass interference filter at $250 \mathrm{~nm}$, corresponding to transitions in the $A^{2} \Sigma^{+}$ $\left(v^{\prime}=0\right) \leftarrow X^{2} \Pi\left(v^{\prime \prime}=2\right)$ band.

Shown in Fig. 1 are the results of DFWM intensity measurements (filled symbols) for various mixtures and for two different pumpbeam intensi:ies, corresponding to $I / I_{\text {sat }}=0.02$ (upper panel) and $I / I_{\text {sat }}=0.5$ (lower panel). (Saturation values are given for the 1:1 mixture, since $I_{\text {sat }}$ increases by approximately $50 \%$ from left to right in the figure.) The measurements at the left side of the plot correspond to 100 Torr of pure $\mathrm{N}_{2}$ while those at the right side correspond to 96.6 Torr of pure $\mathrm{CO}_{2}$. For both values of $I / I_{\text {sat }}$ the signal intensity is observed to fall with increasing $\mathrm{CO}_{2}$ mole fraction, i.e., with increasing quenching. For the upper panel, the intensity decreases by only about $50 \%$ even though the quench rate increases by more than $10^{3}$. The signal decrease is even smaller for the more strongly saturated case, approximately $15 \%$. This result demonstrates that DFWM signals are indeed relatively insensitive to quench rate[2] in the population-grating regime; similarly measured LIF intensities (not shown) decreased by a factor of 7 to 350, depending on the degree of saturation. 


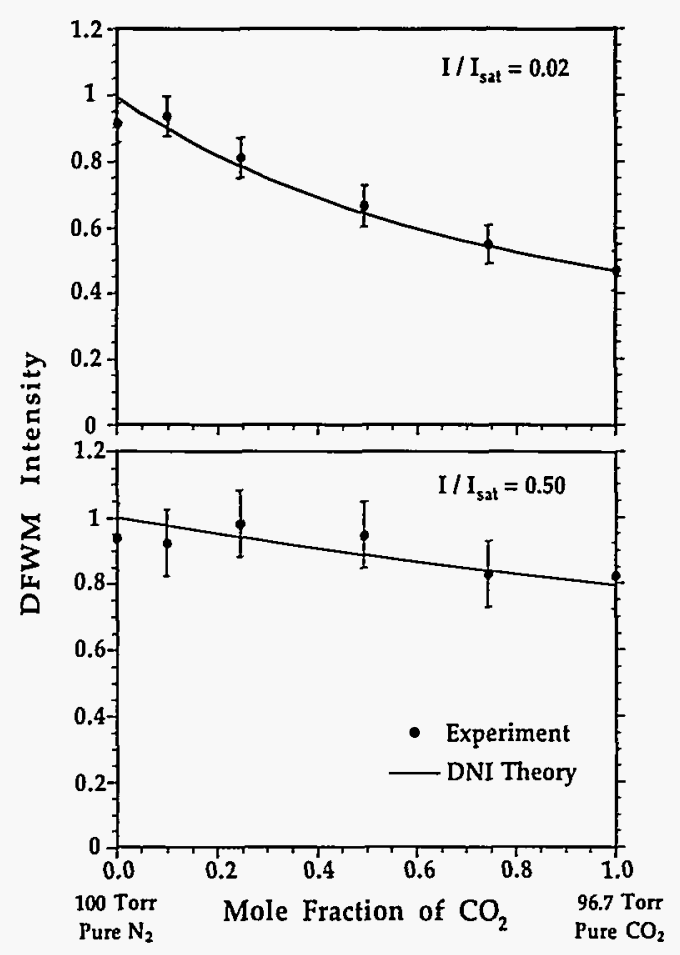

Figure 1. Experimental and theoretical DFWM intensities of a fixed density of $\mathrm{NO}$ plotted against mole fraction of $\mathrm{CO}_{2}$ in an $\mathrm{N}_{2} / \mathrm{CO}_{2}$ mixture. The NO quench rate increases by more than $10^{3}$ from left to right, while the collisional broadening $\gamma_{12}$ is held fixed at 0.0385 $\mathrm{cm}^{-1}$ (possible because the broadening coefficients for $\mathrm{NO}$ in $\mathrm{CO}_{2}$ and $\mathrm{N}_{2}$ are similar). DNI refers to direct numerical integration of the velocity-dependent density matrix equations. ${ }^{3}$

At higher pressures in a quenching buffer gas, molecular collisions convert the NO Astate population gratings into thermal gratings (TG). The sudden addition of heat results in bulk pressure and density perturbations, both of which cause perturbations in the index of refraction. The index-of-refraction gratings result in diffraction of signal beams that cannot be easily distinguished from population-grating contributions in ordinary DFWM experiments.

Using a second laser to probe the grating at varying times after its excitation, a distinctive, long-lived temporal signature characteristic of TG scattering can be observed. Shown in Fig. 2 is a backwardpump time-delay scan performed with 40 ppm of $\mathrm{NO}$ in 1000 Torr of $\mathrm{CO}_{2}$, exhibiting

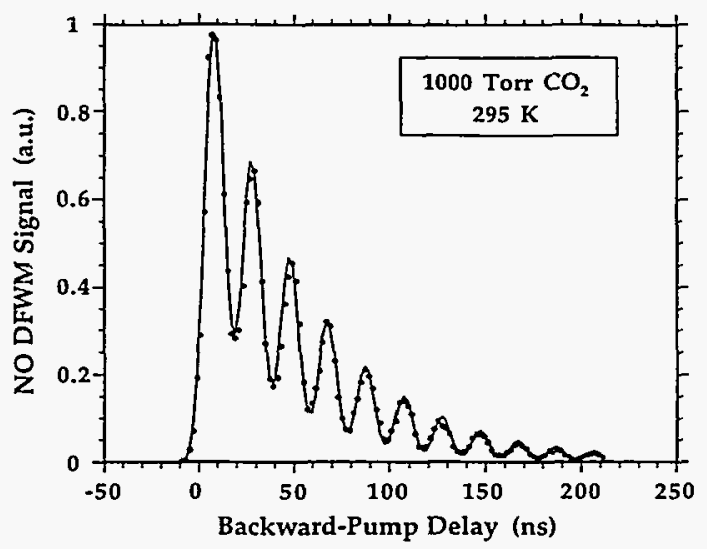

Figure 2. Temporal behavior of thermalgrating scattering in DFWM. Data points are intensity measurements from $40 \mathrm{mTorr}$ of $\mathrm{NO}$ in 1000 Torr of $\mathrm{CO}_{2}$, as a function of time delay between the grating-forming laser pulses and the probing pulse. The solid line is a hydrodynamics calculation.

pronounced oscillations superimposed on a slow decay. The beats result from the excitation of standing acoustic waves by the previously mentioned pressure perturbations; as the waves counter propagate through a fixed point in space the pressure fluctuations modulate the DFWM scattering efficiency. The solid curve in Fig. 2 is a calculation based on a one-dimensional solution of the linearized hydrodynamics equations for describing the induced density, pressure, and temperature fluctuations. A single-pulse measurement of this type could serve as a useful diagnostic of gas-dynamic properties such as speed-ofsound, viscosity, and thermal diffusion rates.

This work was supported by the Division of Chemical Sciences, Office of Basic Energy Sciences, U. S. Department of Energy, and the Office of Industrial Technologies, Division of Advanced Industrial Concepts. Partial funding for PMD was provided by the Air Force Office of Scientific Research, Aerospace Sciences Directorate.

\section{References}

[1] Farrow, R. L. and Rakestraw, D. J. Science 1894 (1992).

[2] Dreier, T. and Rakestraw, D. J. Opt. Lett. 15, 72 (1990).

[3] Lucht, R. P., Farrow, R. L. and Rakestraw, D. J. Opt. Soc. Am. B. 10, 1508 (1993). 


\title{
Full Characterization of Ultrashort Laser Pulses
}

\author{
We have developed a technique for measuring the time-dependent intensity and \\ phase of a single ultrashort laser pulse. A spectrogram of the pulse is measured \\ followed by extraction of the pulse characteristics using a two-dimensional-phase- \\ retrieval computer algorithm.
}

\section{R. P. Trebino, K. W. DeLong, D. N. Fittinghoff, and C. L. Ladera ${ }^{1}$}

Ultrashort laser pulses are the shortest technological events ever created by man. Less than one millionth of a millionth of a second long, they provide high temporal resolution for measuring the many fundamental events that occur on ultrafast time scales. Specifically, intramolecular motion on such short time scales plays an important role in combustion chemistry. In addition, very fast semiconductors, biological media, and a wide variety of physical processes contain phenomena that occur on such tiny time scales. Ultrashort laser pulses make these measurements possible, provided that the pulse is shorter than the event to be measured.

In all of these fields, the difficult problem of measuring the time-dependent intensity and phase of these pulses is critical to the success of any experiment. Unfortunately, it would appear that such a measurement would require an even shorter pulse. This would be a problem because ultrashort laser pulses are already the shortest events ever created. Many years ago, it was realized that the best one could hope to do was to use the pulse to measure itself. This has not been sufficient, and such measurements have yielded only the pulse intensity autocorrelation, a smeared out version of the pulse intensity. In addition, the only phase information available for ultrashort pulses was that in the pulse spectrum. These two measures do not uniquely determine the pulse; indeed, they allow infinitely many possible pulse shapes.

We have solved this problem with a technique that we call Frequency Resolved
Optical Gating[1], or FROG, for short. FROG yields the time-dependent intensity and phase using a simple apparatus. It can measure ultrashort laser pulses in the visible, the infrared, and the ultraviolet, allowing both very complicated, as well as simple, pulses. It can even measure a single ultrashort pulse; it doesn't require averaging over many laser shots, as most laser techniques do. And it works for even the shortest pulses; recently it was used to measure pulses as short as $9 \times 10$ ${ }^{15}$ seconds long.

Like previous methods, FROG uses the pulse to measure, or "gate," itself, but it also incorporates the concept of a "spectrogram" of the optical pulse, analogous to a musical score or graphic-equalizer display of a sound wave. If $E(t)$ is the complex electric field of the pulse vs. time and $g(t-\tau)$ is a variable-delay gate function, the "FROG trace" is given by:

$I_{F R O G}(\omega, \tau) \propto\left|\int_{-\infty}^{\infty} E(t) g(t-\tau) \exp [-i \omega t] d t\right|^{2}$

The FROG trace displays the optical spectrum of temporal slices gated out of the pulse vs. time. In FROG, the gate is typically proportional to the pulse intensity, $|E(t-\tau)|^{2}$, or the pulse field itself, $\mathrm{E}(\mathrm{t}-\tau)$.

FROG traces are easily measured using the apparatus shown in Fig. 1. Central to this apparatus is a nonlinear-optical medium in which the beams cross. A signal pulse is created by the spatial and temporal overlap of

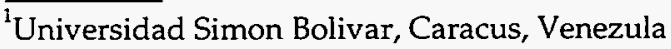




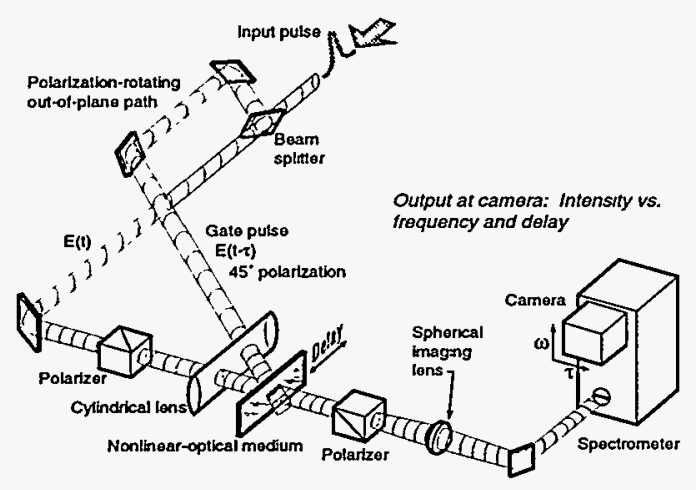

Figure 1. Experimental FROG apparatus for the measurement of a single ultrashort laser pulse.
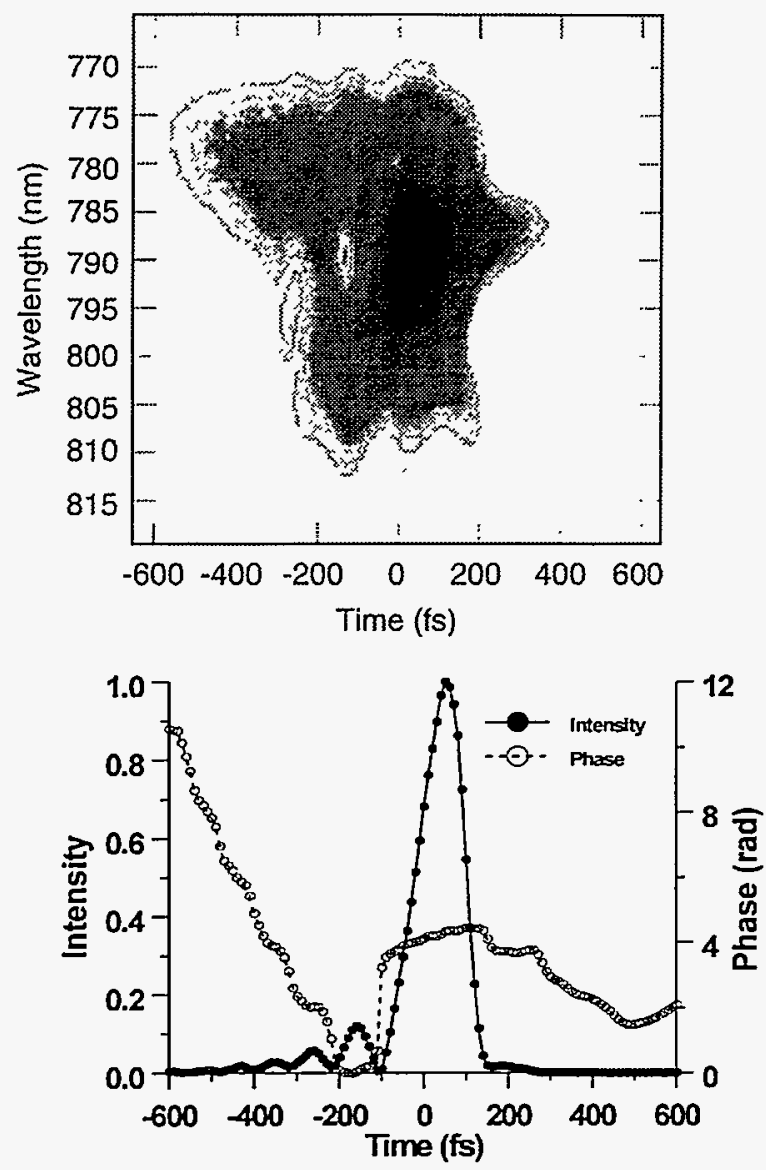

Figure 2. FROG trace of a complex pulse with phase distortions (top). A smooth pulse, on the other hand, would have a trace with elliptical contours of equal intensity. The intensity and phase of the same pulse (bottom). Note the complex time dependence of the intensity and phase. beams in this medium. The relative delay between the two replicas of the pulse is mapped onto position in the nonlinear-optical medium. This is accomplished by crossing the beams at a large angle and focussing with a cylindrical lens instead of a spherical lens.

The FROG trace (Fig. 2) is a very intuitive measure of the pulse. It is best displayed as a density plot of intensity, vs. frequency, $\omega$, and delay, $\tau$. As in a musical score, the FROG trace provides a visual display of the pulse frequency vs. time. In addition, the widths of the trace in the delay and frequency directions roughly indicate the pulse width in time and the pulse spectrum in frequency, respectively. The problem of extracting the pulse from its FROG trace can be shown to be mathematically equivalent to "twodimensional phase retrieval," a solved problem in the field of image science. We have modified two-dimensional phase-retrieval algorithms from image science and applied them to FROG, where they work exceedingly well.

FROG has already been applied to the measurement of shaped laser pulses used by chemists who are researching ways to control chemical reactions[2]. FROG has also been applied to the alignment of laser-pulse compressors, the characterization of $\mathrm{XeCl}$ amplifiers, and diagnostics of laser-induced plasmas. In view of this interest, Sandia has transferred this technology to industry, where FROG is now a commercial product of ClarkMXX.

This work supported by the Division of Chemical Sciences, Office of Basic Energy Sciences, U.S. Department of Energy.

\section{References}

[1] D.J. Kane and R. Trebino, "Single-Shot Measurement of the Intensity and Phase of an Arbitrary Ultrashort Pulse by Using FrequencyResolved Optical Gating," Optics Letters, vol. 18, no. 10, pp. 823-5, 1993.

[2] B.Kohler, V.V. Yakovlev, K.R. Wilson, J.Squier, K.W. DeLong, and R.Trebino, "Phase and Intensity Characterization of Femtosecond Pulses from a Chirped Pulse Amplifier by Frequency-Resolved Optical Gating," Optics Letters, in press. 


\title{
Raman Lidar Profiling of Atmospheric Water Vapor
}

\author{
Raman lidar is a powerful technique for profiling atmospheric water vapor and aerosols. \\ We are developing two Raman lidar systems, one for use in our own research at Sandia, \\ and one that will reside permanently at the DOE climate study site in Oklahoma.
}

\section{J. E. M. Goldsmith, S. E. Bisson, F. H. Blair, and M. G. Mitchell}

Detailed measurements of the distribution of water vapor in the atmosphere are needed for a variety of scientific inquiries, including global climate change and related issues in radiative processes (water vapor is the major greenhouse gas in the atmosphere), and studies of a variety of atmospheric processes such as cloud formation and atmospheric circulation. The Raman lidar is a leading candidate for an instrument capable of the detailed, time- and space-resolved measurements required by these and other studies [1].

Raman lidar operates by sending out a laser pulse and recording the atmospherically backscattered return signal as a function of time to provide range information. The return signal consists of elastically scattered laser light which is useful for profiling cloud heights, aerosols, and the planetary boundary layer, and inelastically scattered light that provides chemically specific concentration profiles for species such as water vapor. The inelastic scattering utilized here is the result of the rotational-vibrational Raman effect that shifts the incident wavelength by a frequency characteristic of the molecule $\left(3652 \mathrm{~cm}^{-1}\right.$ for water vapor and $2331 \mathrm{~cm}^{-1}$ for molecular nitrogen). Simultaneous measurement and subsequent ratioing of the water vapor and nitrogen Raman signals provides a quantitative measurement of the water vapor mixing ratio (grams of water vapor per kilogram of dry air). Ratioing of the elastic backscatter and nitrogen Raman signals provides a quantitative measurement of the aerosol scattering ratio. The capabilities of Raman lidar systems have steadily improved by taking advantage of technological advances, particularly those in laser systems and narrowband, high transmission interference filters.
While Raman lidar is used currently to perform meteorologically important, sustained, reliable nighttime profiling of water vapor, daytime measurements present added challenges because of the difficulties inherent in detecting weak Raman signals against solar backgrounds. In our studies of two approaches for obtaining enhanced daytime operation, namely solar-blind operation and narrowband, narrow-field-of-view operation, computer models indicated that comparable daytime performance can be anticipated using the two approaches. Because the latter approach does not degrade the nighttime performance of the lidar system, we have chosen to base systems on a narrowband, narrow-field-of-view design. In order to provide both improved short-range measurements and extended dynamic range, the systems also incorporate a channel with a wider field of view, and hence the description as dual-field-of-view systems.

We are currently developing two Raman lidar systems. One, which has been under development for several years, is used for our own studies at Sandia. The Sandia lidar system is housed in two mobile semitrailers, one trailer serving as a mobile laboratory and the other as a support vehicle providing a data acquisition/analysis area. The lidar uses an injection-seeded excimer laser to provide a beam with reduced divergence and spectral bandwidth, operated at $308 \mathrm{~nm}$ during both nighttime and daytime. Enhanced dynamic range, for daytime operation in particular, is provided by using photon counting in the narrow field-of-view channel, and analog to digital conversion in the wide field-of-view channel. This system has been operational since the summer of 1992, and (as of the writing of this article) has participated in two major field campaigns. 


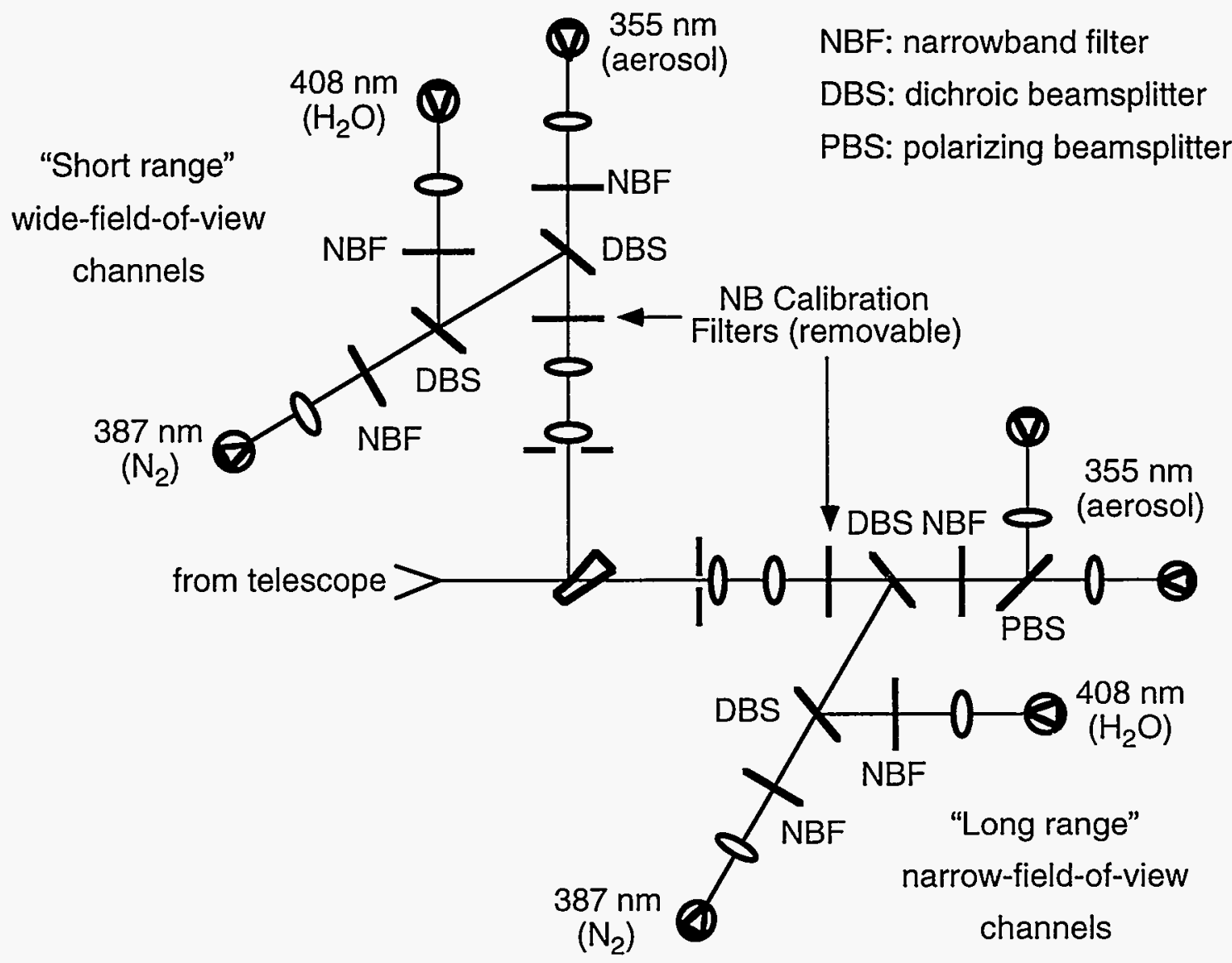

Figure 1. Schematic diagram of the detection system in the CART Raman lidar system.

The second Raman lidar system under development will have a permanent residence at the Department of Energy's ARM (Atmospheric Radiation Measurement program) CART (Cloud and Radiation Testbed) site near Lamont, Oklahoma [2]. This system is based on a high-power 355-nm laser beam produced by a frequency-tripled Nd:YAG laser. Fig. 1 displays a schematic diagram of the detection system that is located after the receiving telescope. (The detection system in the Sandia lidar is very similar, but does not have a polarization-sensitive aerosol channel, and operation with the $308 \mathrm{~nm}$ excimer laser produces aerosol, nitrogen, and water vapor signals at $308 \mathrm{~nm}, 332 \mathrm{~nm}$, and $347 \mathrm{~nm}$, respectively.) The CART system is housed in an environmentally hardened $8^{\prime} \times 8^{\prime} \times 20^{\prime}$ shipping container. Because it will be operated routinely in a "field" environment, substantial efforts have been devoted to making its operation as turn-key as possible, including computer-automated laser operation, alignment of the transmitted laser beam, system calibration, operation of all electronics, and data handling and processing. We anticipate that this system will provide key measurements for a wide variety of climate studies.

This work supported by the U.S. Department of Energy, Atmospheric Radiation Measurement (ARM) Program.

\section{References}

[1] J. E. M. Goldsmith, Scott E. Bisson, Richard A. Ferrare, Keith D. Evans, David N. Whiteman, and S. H. Melfi, "Raman Lidar Profiling of Atmospheric Water Vapor: Simultaneous Measurements with Two Collocated Systems," Bull. Amer. Meteor. Soc. 75, 975 (1994).

[2] J. E. M. Goldsmith, S. E. Bisson, and F. H. Blair, "Implementation of Raman Lidar for Profiling of Atmospheric Water Vapor and Aerosols at the SGP CART Site," in Proceedings of the Fifth Atmospheric Radiation Measurement (ARM) Science Team Meeting (in press). 


\title{
The Development of a Laser-Based Imaging System For Long-Range Gas Plume Visualization
}

\author{
Backscatter Absorption Gas Imaging (BAGI) is a laser remote sensing technique that allows \\ real-time video visualizations of gas plumes. A new BAGI imager has been developed that \\ allows imaging at ranges over a factor of two greater than past devices.
}

\section{T. J. Kulp and R. Kennedy}

The Backscatter Absorption Gas Imaging (BAGI) technique is a video gas plume visualization method that is accomplished using laser active imaging. The application of plume imaging to the detection of fugitive emissions is a new approach that has many distinct advantages over traditional point or line-of-sight sensing methods. Imaging allows (1) rapid searching of large areas for leak sources, (2) ease of identification of both the presence and location of a gas leak by an untrained operator, and (3) less sensitivity to system constraints (such as laser power stability and wavelength stability) that commonly affect instruments based on quantitative signals. Prior applications of imaging to gas detection have been carried out using passive detection in the infrared (IR). While successful, passive IR imaging is limited in sensitivity because it requires a temperature difference between the scene and the plume to be detected. BAGI overcomes this limitation by imaging a scene as it is illuminated by laser radiation of a wavelength coincident with an absorption band of the gas to be visualized. Gas plumes present in the scene become visible in the real-time video image when they attenuate a portion of the back-scattered radiation (see Fig. 1).

We describe here the design and preliminary performance evaluation of a new BAGI imager that is intended for long-range operation at target ranges of up to $300 \mathrm{~m}$. Such a system would allow airborne gas imaging from a low-flying airplane or helicopter. Like previous BAGI instruments, this device operates in the IR and images gases via absorption by their fundamental vibrational bands. It accomplishes this using a $\mathrm{CO}_{2}$ laser, which emits wavelengths in the range between 9 and $11 \mu \mathrm{m}$, allowing over 80 different organic species to be detected. Prior imagers have also used $\mathrm{CO}_{2}$ lasers or have used a helium neon laser operating at $3 \mu \mathrm{m}$, and have been limited to ranges of 6 to $110 \mathrm{~m}$. The devices that have been used to accomplish BAGI consist of a raster-scanning infrared imaging video camera that is coupled to a continuous wave (CW) IR laser. Raster scanned imaging is achieved using a pair of synchronized galvanometer-driven scan mirrors. The concerted motion of the mirrors sweeps the instantaneous field-of-view (IFOV) of an IR detector and the beam of the laser source across the target in a raster pattern. The laser backscatter signal that is received is formatted to generate a real-time RS- 170 video image.

In considering the design of a long-range mid-IR imager, one must select between heterodyne and direct modes of detection. The former offers the possibility of shot-noiselimited signal-to-noise ratios, whereas the latter is background limited. Thus, for the same laser power, much greater range can be achieved with heterodyne imaging. Heterodyne sensing is a coherent technique that requires the detection of backscatter having a uniform phase front. This limits the size of the receiver aperture that can be used and increases laser speckle in the image. It also places severe alignment constraints on the system. These practical limitations, and the fact that only a modest range extension was required led us to choose direct detection for the long-range imaging system.

Improvements to the imager range have been formulated using a standard lidar signal model in conjunction with data from past field tests. The range performance of the past BAGI imagers was limited by the small size $(1.2 \mathrm{~cm})$ 
of its receiver aperture. The long-range imager was designed to operate with $5 x$-magnification telescopic optics to provide a corresponding expansion of the receiver and transmitter diameters. To introduce these optics into the system without generating unwanted scatter crosstalk between the transmitting and receiving paths, a separated-path scanner design was formulated.

The optical design that was constructed is shown in Fig. 2. Refractive optics are used in the transmit and return telescopes. To separate the paths, the laser is scanned using a polished surface on the rear of the horizontal scan mirror whereupon it is directed down to the vertical mirror. The IFOV is scanned, as in the past, from the front surface of the horizontal scan mirror and directly downward to the vertical mirror. The vertical scan mirror was enlarged to accommodate the footprints of the laser and IFOV in two distinctly separated regions. In addition, baffles (not shown in the diagram) are used to optically isolate the laser transmit and return paths from each other. Upon leaving the vertical mirror, the laser and IFOV paths enter their respective telescopes. Overlap of the laser and IFOV at the target is accomplished by translating the IFOV objective lens to aim the IFOV at the appropriate region on the target plane. The laser used in the prototype imager is a beam-expanded 20$\mathrm{W}$ tunable waveguide $\mathrm{CO}_{2}$ system.

The long-range BAGI imager was first tested as a breadboard prototype during the fall of 1992 at Lawrence Livermore National Laboratory. During the time period of 1993-4, a packaged prototype imager was fabricated and testing of that system is currently underway at Sandia. As in earlier field tests, images were made of a variety of sulfur hexafluoride releases against target panels having known IR reflectivities. Video images were collected on an ordinary VHS videocassette recorder. During the tests, gas imaging was performed at ranges up to $300 \mathrm{~m}$ $(1000 \mathrm{ft})$. Preliminary results indicate that an acceptable signal-to-noise ratio is achievable at ranges up to about $227 \mathrm{~m}(750 \mathrm{ft})$. Beyond that point, a notable deterioration in image quality is encountered, resulting primarily from image intensity variations caused by the atmospheric turbulence modulation of laser speckle. We are presently continuing the field experiments and will be considering methods of reducing the effects of turbulence and speckle. The development of the long-range imager has been carried out for the US Navy and is expected to be complete in mid- 1995. Work is also beginning on a new imaging system that will use a pulsed 3- $\mu \mathrm{m}$ laser in conjunction with a focal-plane-array detector to image natural gas leaks.

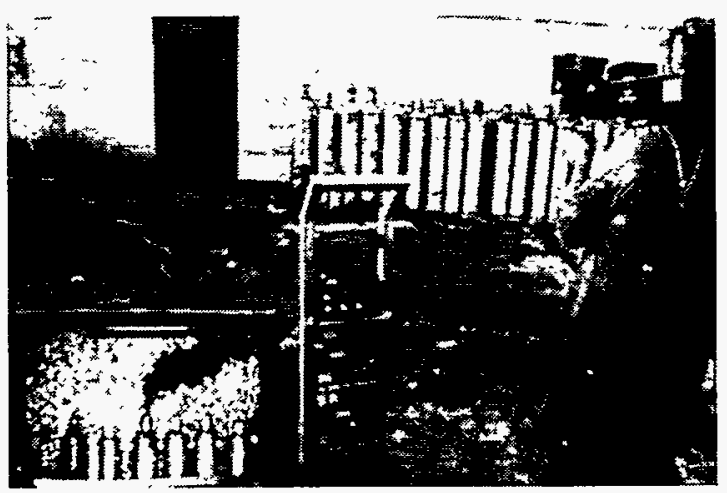

Figure 1. Photograph illustrating the concept of the BAGI technique. The operator is demonstrating the first BAGI imager (developed at Lawrence Livermore National Laboratory), having an imaging range of 30 $\mathrm{m}$, as it is imaging a freon leak.

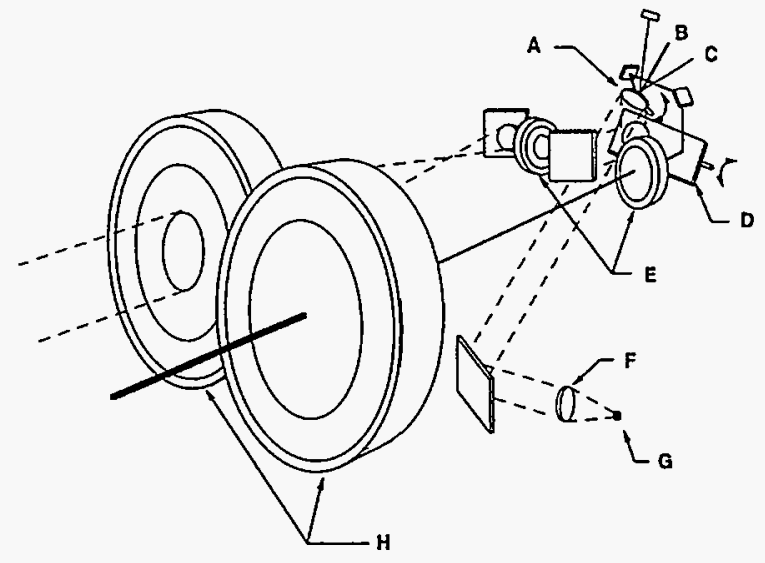

Figure 2. Diagram of the optical layout of the long-range BAGI imager. Components are: (A) horizontal scan mirror; (B) laser beam input; (C) position sensor input; (D) vertical scan mirror; (E) telescope eyepiece elements; (F) lens; (G) $\mathrm{HgCdTe}$ detector; and (H) telescope objective lenses.

This work supported by the U. S. Navy, Office of Salvage and Diving, Naval Sea Systems Command. 



\section{Combustion Chemistry}

\section{Introduction}

Under the principal sponsorship of the Department of Energy's Office of Basic Energy Sciences, Sandia National Laboratories undertakes fundamental experimental and computational research in combustion chemistry. This work provides information needed to monitor and understand the complex chemical processes that occur when fuels burn. The program emphasizes determination of the rates and mechanisms of chemical reactions, characterization of molecular structure and energetics, and measurement and modeling of idealized combustion devices. The research utilizes state-of-the-art experimental and computational techniques and stresses close coupling among experiment, theory, and modeling.

Staff and visitors investigate processes important in fuel combustion, pollutant formation, and energetic-materials decomposition. Unimolecular, bimolecular, and termolecular chemical reactions drive these phenomena. Experimentally, laser-based pump/probe techniques interrogate chemical reactivity and structure at several levels. Ionimaging techniques permit the simultaneous measurement of velocity and internal-state distributions of reaction products formed in single-collision encounters. Femtoseconddomain experiments probe directly the conversion of energy from one form to another within a molecule. Advanced, non-linear optical techniques facilitate spectroscopic characterization of critical combustion intermediates. Photolysis/fluorescence and dischargeflow/mass spectrometry methods determine the thermal rate coefficients and product distributions for reactions that constitute the individual steps in the combustion chain process. Thermogravimetric/mass-spectrometric experiments characterize thermal decomposition pathways of energetic materials. Lowpressure flame measurements elucidate the dominant reaction pathways in combusting systems. Current computational efforts include $a b$ initio calculations of molecular energetics and computer modeling of the complete kinetics of combustion processes in both laminar and turbulent environments. 


\title{
Ion Imaging of Unimolecular and Bimolecular Reaction Products
}

\author{
The Ion Imaging technique enables the study of many types of unimolecular and bimolecular \\ chemical processes. Information about the velocity, state distribution, alignment and \\ timescale of processes is derived from analysis of the ion images.
}

\section{W. Chandler, T. Kitsopoulos, R. McKay1, A. Heck1, A. Schiffman, M. Jaska and R. N. Zare1}

In recent years the development of new techniques to study gas phase reaction dynamics of 'full' and 'half' collisions has been tremendous. The ultimate goal in this field of research is to achieve control of chemical reactions but first we must study them in detail. Levine and Bernstein ${ }^{(1)}$ have outlined 'desiderata' for the study of molecular reaction dynamics stating that "in the ultimate experiment one would determine the cross section and angular distribution of the products for a completely specified collision... total reaction cross section at specified collision energies, angular distribution of products, internal state analysis of the products, internal state selection of reactants, selection of orientation or of alignment of reactants and analysis of the alignment of the products". So far no experiment has simultaneously implemented all of these 'desiderata' .

The majority of chemical dynamics studies focus on the asymptotic properties of the reaction, measuring the quantum-state distributions or velocity distributions of the products. Ion imaging( 2 combines resonance enhanced multiphoton ionization (REMPI) techniques with time-of-flight (TOF) techniques in a way that provides for stateselective detection and determination of the velocity and angular distributions of the products.

The ion-imaging apparatus is conceptually straight forward and is shown in Figure 1. A photolyte is expanded supersonically through a pulsed valve and introduced into the first

${ }^{1}$ Stanford University, Stanford, California stage of a differentially pumped vacuum chamber. The ensuing molecular beam is collimated by a single skimmer before entering the reaction and detection region, part of the second stage of the vacuum chamber. A few centimeters downistream the molecular beam passes through a hole in the repeller plate. Approximately halfway between the repeller and extractor plate the skimmed molecular beam is intersected by one or more linearly polarized laser beams, with propagation direction perpendicular to the molecular-beam propagation axis. The polarization direction of the photolysis-laser beam defines an axis of cylindrical symmetry for the recoil of the photofragments. Fragments generated in the photodissociation are quantum-state selectively ionized by the ionization laser. Upon ionization the ions are accelerated along the molecular-beam propagation axis by the static field between the repeller and extractor plate toward the time-of-flight (TOF) field-free region. At the end of the TOF region the ions impinge upon the position-sensitive detector. In order to detect the ions of interest mass selectively the front plate of a microchannel plate is pulsed to a negative voltage upon arrival of the ions of interest thereby ensuring maximum gain of the detector for the ions of interest only. Mounted behind the microchannel plates assembly is a fast phosphor screen. Ion images appearing on the phosphor screen are recorded by imaging the output of the fiberoptic bundle onto a cooled charge-couple device (CCD) camera using a standard camera lens. Signal averaging is accomplished by keeping the camera shutter open for a long period and averaging the signal on the CCD. 


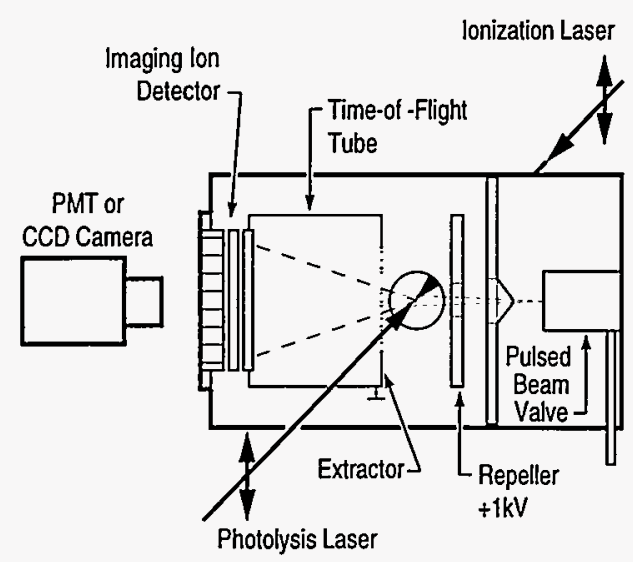

Figure 1. Schematic of ion imaging apparatus used to study unimolecular dissociation reactions.

The recorded "Ion Images" are twodimensional projections, along an axis perpendicular to the plane of the detector, of the three-dimensional distribution of ionized fragments. If the initial three-dimensional distribution has cylindrical symmetry, a unique transformation, the inverse Abel transform, can be used to reconstruct from the two-dimensional images intensity cross sections through the center of the initial three dimensional distribution. There is a unique relationship between the cylindrically symmetric two-dimensional functions and their line integrals which enables us to reconstruct from the data angular distributions for both photofragmentation $\left(\mathrm{CH}_{3} \mathrm{I}, \mathrm{C}_{2} \mathrm{H}_{2}, \mathrm{CH}_{3} \mathrm{Br}, \mathrm{HI}, \mathrm{H}_{2}\right.$ and $\left.\mathrm{C}_{6} \mathrm{H}_{8}\right)$ and bimolecular reaction $\left(\mathrm{H}+\mathrm{D}_{2}\right.$ and $\left.\mathrm{H}+\mathrm{HI}\right)$ scattering processes.

A large amount of information is available from analysis of a single reconstructed image. Every point in the reconstructed image is associated with three parameters: radius (distance from the center-of-mass of the reaction), angle (relative to the symmetry axis) and intensity. The radial position is a direct measure of the translational energy of the species, which can be coupled to the known energetics of the system to elucidate the reaction channels, or conversely, to derive system energetics. Image intensities give a direct measure of the population, or number density, for each product energy and angle. The spatial distributions measured by imaging techniques make it possible to distinguish different reaction channels contributing to the same product, and relative branching ratios between these different channels can be measured. The resonant nature of laser-based detection allows quantum-state resolution in the product detection. Comparison of ion images corresponding to different product quantum states, allows the measurement of state-resolved relative reaction cross-sections.

An example of a simple unimolecular dissociation process is the fragmentation of the $\mathrm{KrAr}$ dimer during a $2+1$ REMPI process. Two-photon resonances of the $\mathrm{KrAr}$ dimers are observed near $212 \mathrm{~nm}$. In the TOF both $\mathrm{KrAr}^{+}$ions and $\mathrm{Ar}^{+}$ions are observed. The image seen when the $\mathrm{Ar}^{+}$ions arrive at the detector is shown in Figure 2. From inspection of the image we learn that there are two $\mathrm{Ar}^{+}$ production mechanisms, one giving very little kinetic energy, correlating with absorption of two photons producing electronically excited $\mathrm{Ar}$, and one producing very fast $\mathrm{Ar}^{+}$ correlating with absorption of a third photon prior to dissociation.

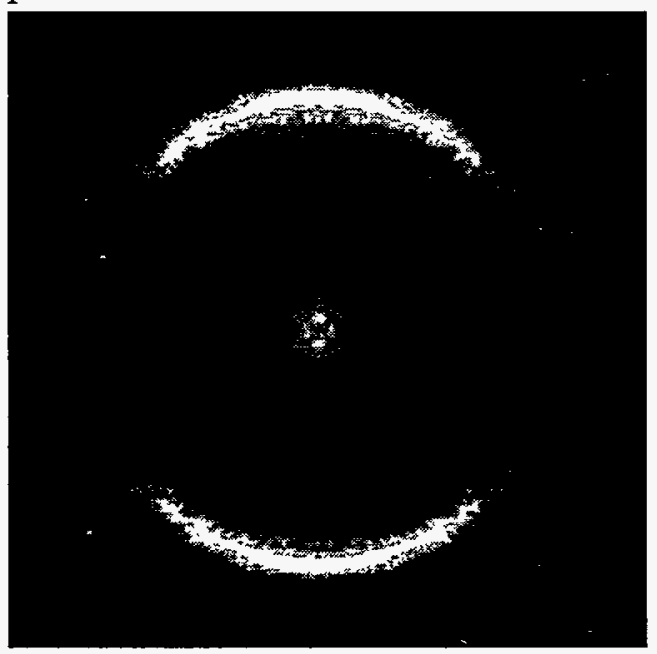

Figure 2 . Image of $\mathrm{Ar}^{+}$ions resulting from the two-photon dissociation of KrAr dimers.

This work was supported by the Division of Chemical Sciences, Office of Basic Energy Sciences, U.S. Department of Energy.

\section{References}

[1]] Levine, R.D., Bernstein, R.B., Molecular Reaction Dynamics. 1987, Oxford: Oxford University Press.

[2] Chandler, D.W., Houston, P. L. 1987. J. Chem. Phys. 87: 1445-1447, Kitsopoulos, T.N., Buntine, M. A., Baldwin, D. P., Zare, R. N., Chandler, D. W. 1993. Science 260: 1605-1610 


\title{
Femtosecond Laser Studies of Ultrafast Photochemical Processes
}

\author{
Femtosecond laser studies using photoionization mass spectrometry reveal details of ultrafast \\ nonradiative processes in highly excited molecules
}

\section{C. Hayden, D. W. Chandler, M. A. Gutzler and D. R. Cyr}

The conversion of energy from one form to another within a molecule provides the driving force for many chemical processes. These energy transfer processes can occur on a time scale of $10^{-13}$ sec. (100 femtoseconds), only a few periods of the vibrations in a molecule. Only with the development of femtosecond lasers has it become possible to observe these fast processes directly. A particularly important process of this type, known as internal conversion, is the conversion of electronic excitation, produced by optical absorption, to vibrational excitation within molecules. Ultrafast internal conversion produces highly vibrationally excited molecules that are similar to intermediates in radical-radical reactions occurring during combustion. The molecules of interest in these studies undergo initial steps of internal conversion in less than 100 fsec. By initiating ultrafast internal conversion with femtosecond laser pulses we can efficiently generate highly vibrationally excited molecules on a $100 \mathrm{fsec}$ time scale and, using femtosecond probe pulses, study their intermolecular dynamics at energies that are well above the energies required for isomerization and dissociation. A long term goal of these studies is to measure the time scale for redistribution of vibrational energy throughout molecules, because it is this energy redistribution that ultimately determines the pathways of many chemical reactions.

Rapid internal conversion is also a fundamentally important process in organic photochemistry. The higher electronic states of most organic molecules do not fluoresce because fast internal conversion converts the electronic energy into vibrational energy. These vibrationally hot products undergo subsequent chemical reactions that form the basis for much of organic photochemistry. Two notable examples where ultrafast internal conversion plays an important role are the chemical processes that follow light absorption in vision and photosynthesis. In fact, the 1,3,5-hexatriene system that is the subject of our current studies has served as a model for understanding initial steps in the photochemistry involved in vision and photosynthesis. 1

A multiple wavelength femtosecond laser system is the key experimental component in these studies. The laser system is designed to be tunable over a very wide wavelength range. For the experiments described here the laser system is configured to produce precisely synchronized pulses at $250 \mathrm{~nm}$ and $350 \mathrm{~nm}$ with pulse lengths of less than 200 femtoseconds. Femtosecond pulses are initially produced at $620 \mathrm{~nm}$ with a collidingpulse mode-locked dye laser. The output of this laser is amplified and used to generate continuum in two separate quartz plates. The continuum outputs around $750 \mathrm{~nm}$ and 700 $\mathrm{nm}$ are further amplified and pulse compressed. An excitation pulse at $250 \mathrm{~nm}$ is produced by generating the third harmonic of the $750 \mathrm{~nm}$ pulse, while the $350 \mathrm{~nm}$ probe pulse is the second harmonic of the $700 \mathrm{~nm}$ pulse, as shown in Figure 1.

Experiments on ultrafast internal conversion of 1,3,5-hexatriene are performed in the ionization region of a time-of-flight mass spectrometer. The internal conversion process is probed using femtosecond multiphoton ionization. The $250 \mathrm{~nm}$ pulse excites the $\mathrm{S}_{2}$ state in 1,3,5-hexatriene, and the ion yield produced by the $350 \mathrm{~nm}$ ionization pulse is measured as a function of time delay between the laser pulses. With the mass spectrometer, the fragmentation pattern of internal conversion products can be measured 


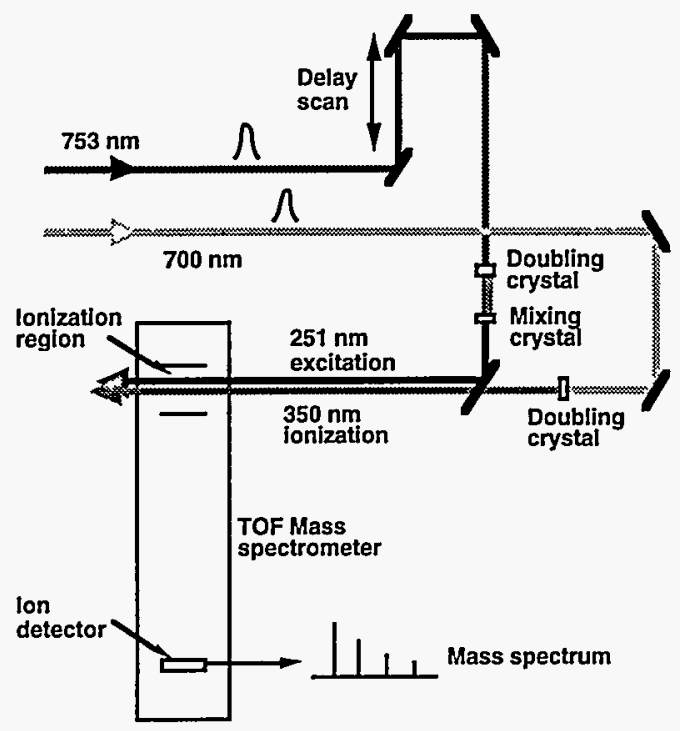

Figure 1 Schematic of the experimental setup for femtosecond time resolved photoionization studies.

as a function of delay after excitation on a femtosecond time scale. In experiments with low intensity $350 \mathrm{~nm}$ ionization pulses only the population of the electronic state initially excited at $250 \mathrm{~nm}$ is observed. The rapid rise and fall of the signal, seen as the solid line in Figure 2, demonstrates that the initial internal conversion process is extremely fast, in fact, faster than the laser pulses.

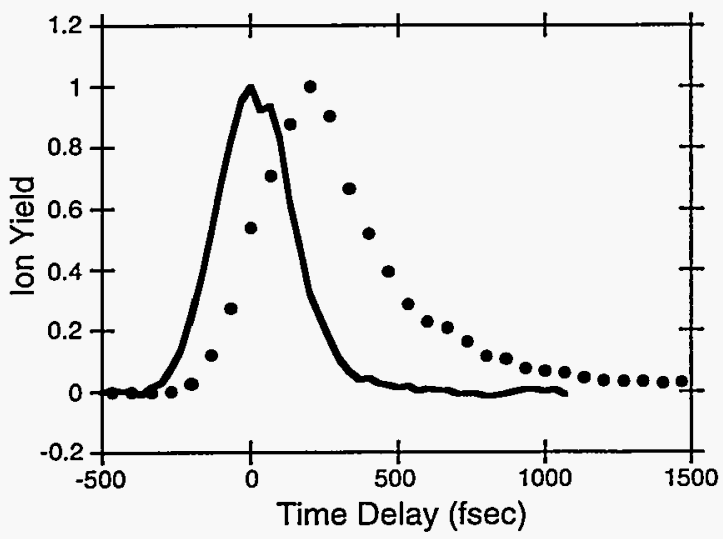

Figure 2. Plot of ion yield vs. delay between excitation and ionization lasers for 1,3,5hexatriene. The solid line shows result of probing the initially excited state; solid points show fragment mass yield, probing a short-lived intermediate state.

When much higher intensity ionization pulses are used multiphoton ionization of the excited molecules can occur, leading to extensive fragmentation. Our experiments show that multiphoton ionization and fragmentation from the initially exited state of the molecule does not occur efficiently. However, the excited molecule evolves through an intermediate state that does produce fragments from multiphoton ionization. The points in Figure 2 show the yield of one of these fragments as a function of time delay. The delayed rise and slower decay is due to the build up and decay of a previously unknown intermediate species in the internal conversion. This is evidence that another electronic state is involved in the process. Model calculations give a $65 \mathrm{fsec}$ rise time constant for the population of the intermediate, with a 250 fsec decay time constant. $^{2}$ The total decay time of the signal demonstrates that redistribution of energy within the molecule continues for more than one picosecond even though the initial step occurs in less than $100 \mathrm{fsec}$.

These results are a start at understanding the dynamics of ultrafast internal conversion. They demonstrate that femtosecond photoionization techniques can follow the energy redistribution in time, far beyond the decay of the initially excited electronic state. We have recently begun to use femtosecond time resolved photoelectron spectroscopy to further characterize intermediates observed in the photoionization experiments. Future experiments will probe the time evolution in more detail, particularly to determine the molecular geometry changes that drive these chemical processes.

\section{References}

[1] B. S. Hudson, B. E. Kohler, and K. Schulten, in Excited States, edited by E. C. Lim, (Academic Press, New York, 1982), Vol. 6.

[2] C. C. Hayden and D. W. Chandler, J. Phys. Chem., submitted. 


\section{Laser-Induced Gratings in Molecular Spectroscopy and Chemical Dynamics}

We are developing two-color resonant four-wave mixing and transient grating techniques for use in molecular spectroscopy and chemical dynamics. Recent applications include spectroscopic studies of $\mathrm{HCO}$ and photofragment transient grating experiments on $\mathrm{NO}_{2}$.

\section{E. A. Rohlfing, T. J. Butenhoff, J. R. Dunlop, J. D. Tobiason, S. Williams, and E. B. Bochenski}

Resonant four-wave mixing (RFWM) is a nonlinear optical technique that has been demonstrated as a spectroscopic and remote sensing technique in a wide range of gas phase environments. In RFWM three laser fields drive a nonlinear polarization in the sample that produces a coherent signal beam. We are particularly interested in two-color RFWM processes, two of which are shown in Fig. 1. RFWM can be qualitatively described with an induced grating picture. Two laser beams of identical frequency $\left(\omega_{1}=\omega_{2}\right)$ are crossed at a small angle and produce a spatially modulated intensity pattern. When the laser frequency is resonant with a molecular transition the intensity modulation is mapped onto a population modulation, or grating, in the ground and excited states. A probe laser beam $\left(\omega_{3}\right)$, tuned to a transition from either of the states involved in the grating-forming step, diffracts off this population grating to produce

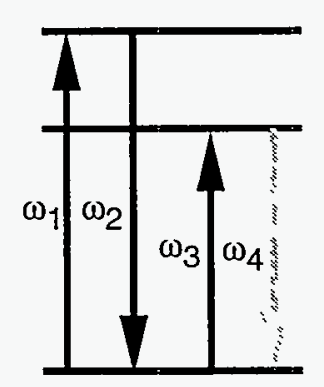

Ground-state grating

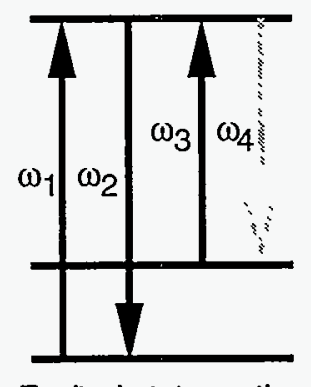

Excited-state grating
Figure 1 Two-color RFWM processes. The left side illustrates diffraction from a groundstate population grating; the right side shows diffraction from an excited-state grating via a downward transition as in RFWM-SEP. the signal beam.

Two-color RFWM is a powerful tool because it enables one to perform backgroundfree double resonance spectroscopy. One example is stimulated emission pumping (SEP) spectroscopy, which is used to characterize ground-state potential energy surfaces. In conventional SEP one laser beam excites the molecule while a second is tuned through downward transitions that terminate on highly excited vibrational levels. The SEP resonances are detected as depletions in the spontaneous fluorescence from the excited state. In RFWM-SEP the probe beam diffracts from the excited-state population grating via the downward transition (Fig. 1). Since the signal beam is generated only when the probe laser is in resonance, RFWM-SEP is a background-free technique.

In Fig. 2 we display examples of RFWMSEP spectra obtained for an important combustion radical, $\mathrm{HCO}$, that has been formed and cooled in a free-jet expansion. These spectra probe a level with four quanta of $\mathrm{C}-\mathrm{O}$ stretching motion that lies above the anomalously low threshold for dissociation into $\mathrm{H}+\mathrm{CO}$. The narrow widths of the rotational lines reveal that this dissociative resonance is long lived and illustrate that the $\mathrm{C}-\mathrm{O}$ stretching motion is not effectively coupled to the $\mathrm{C}-\mathrm{H}$ bond dissociation. The relative intensities of the rotational lines in Fig. 1 change dramatically with changes in the linear polarization states of the four beams involved in the RFWM process. Such effects can be quantitatively accounted for using nonlinear optical perturbation theory and a spherical tensor analysis [1]. 


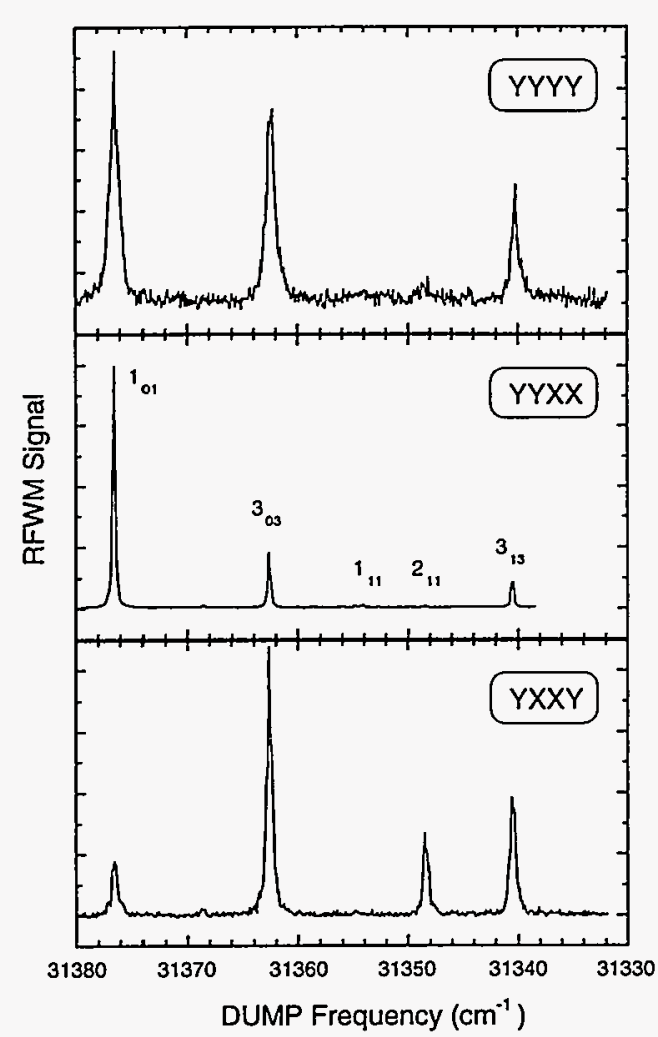

Figure 2. Two-color RFWM-SEP spectra of jetcooled HCO taken in three different linear polarization configurations. These spectra probe a vibrational level in the ground state with four quanta of C-O stretch; each line corresponds to an individual rotational level.

Probing the temporal evolution of the induced grating is referred to as a transient grating experiment. We use a new photofragment transient grating technique to study the nearthreshold photodissociation dynamics of jetcooled $\mathrm{NO}_{2}$ [2]. In these experiments the excited-state grating is converted by dissociation into population gratings in the $\mathrm{NO}$ and $\mathrm{O}$ fragments. Figure 3 displays transient grating signals obtained by probing NO fragments in specific rotational states. Photodissociation produces translationally excited (hot) fragments, some of which move from grating peaks to nulls which leads to a decrease in the diffraction efficiency. As more time passes, the diffraction efficiency may increase as the fragment motion reforms the grating. Thus, the decays in Fig. 3 exhibit oscillatory behavior with the fastest moving fragment, $J "=2.5$, showing the highest oscillation frequency.

In a more quantitative sense, the photofragment grating decay reveals the velocity distribution of the recoiling fragment.

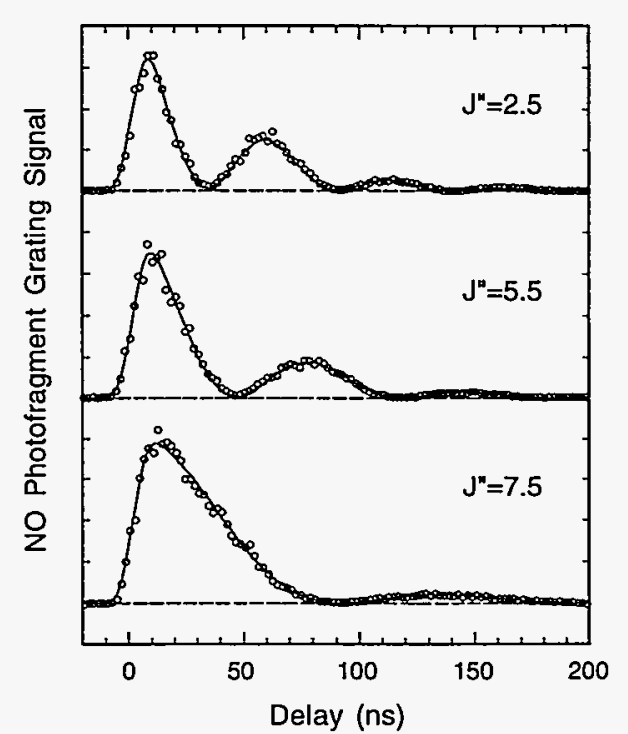

Figure 3. Photofragment transient grating signals obtained for the NO fragments in $J^{\prime \prime}=2.5,5.5$, and 7.5 produced by the photolysis of jet-cooled $\mathrm{NO}_{2}$ at an excess energy of $126 \mathrm{~cm}^{-1}$. The open circles are the data and the solid lines are fits to the photofragment decay that yield the fragment velocity distributions.

Each NO fragment in a particular rotational state has a single speed and the velocity distribution perpendicular to the grating fringes is determined by the angular distribution of the fragment. The data in Fig. 2 is fit to a model that incorporates the fragment velocity distribution into a Fourier transform relationship to obtain the temporal decay of the photofragment grating [2]. The fragment speeds determined from the fits are in excellent agreement with the known speeds. In addition, the fits give the angular distribution of the fragments. The photofragment technique is ideally suited to very slow photofragments, for example, the $\mathrm{J}^{\prime \prime}=7.5 \mathrm{frag}$ ment shown in Fig. 3 has only $7.3 \mathrm{~cm}^{-1}$ of translational energy.

\section{References}

[1] Williams, S.; Tobiason, J. D.; Dunlop, J. R.; and Rohlfing, E. A., "Stimulated Emission Pumping via Two-Color Resonant FourWave Mixing," J. Chem. Phys., submitted, 1994.

[2] Butenhoff, T.J. and Rohlfing, E. A., J. Chem. Phys. 98, 5469 (1993). 


\title{
Kinetics of the $\mathrm{NH}_{2}+\mathrm{NO} \rightarrow$ Products Reaction
}

\author{
We combine experimental studies with theoretical calculations to understand the critical \\ combustion reaction $\mathrm{NH}_{2}+\mathrm{NO} \rightarrow$ Products .
}

\section{J. L. Durant, D. L. Yang, M. Wolf, ${ }^{1}$ H. S. Thiesemann, ${ }^{1}$ and M. Gutzler}

In a 1979 paper, Hack et al noted: "It was a great success when Haber and Bosch first broke the $\mathrm{N}-\mathrm{N}$ bond to produce $\mathrm{NH}_{3}$ from $\mathrm{N}_{2}$ molecules. Nowadays the destruction of $\mathrm{N}_{2}$ and the resulting production of NOx leads to severe problems since the production of energy by fuel-air combustion has increased so rapidly in the last twenty years." 1

The chemistry of $\mathrm{NHx}$ species is intimately tied to that of NOx. In combustion, NHx species are intermediates in the conversion of alkyl fuel bound nitrogen to NOx and $\mathrm{N}_{2}$ products. Ammonia has been used as a flue gas additive to remove NOx in the thermal DeNOx process. Central to this process is the reaction of $\mathrm{NH}_{2}$ with $\mathrm{NO}$, whose major product channel is formation of $\mathrm{N}_{2}$ and $\mathrm{H}_{2} \mathrm{O}$. The minor product channel, $\mathrm{N}_{2} \mathrm{H}+\mathrm{OH}$ formation, acts as a chain branching step, allowing the DeNOx process to continue.

Some appreciation for the complexity of this "simple" bimolecular reaction can be gained by considering the primary product channel, which produces $\mathrm{N}_{2}$ and $\mathrm{H}_{2} \mathrm{O}$ from $\mathrm{NH}_{2}$ and $\mathrm{NO}$ reactants. Association reactions make one bond, abstraction reactions make one bond while breaking one and "complex" concerted fission reactions make two bonds while breaking two bonds. However, the reaction $\mathrm{NH}_{2}+\mathrm{NO} \rightarrow \mathrm{N}_{2}+\mathrm{H}_{2} \mathrm{O}$ breaks all three bonds in the reactants and forms three new bonds in the products!

We are presently focusing our efforts on achieving a better understanding of this complex reaction. Towards this end, we are undertaking parallel theoretical and experi-

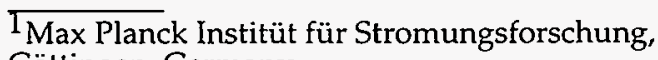
Göttingen, Germany.

${ }^{2}$ Hack, W.; Schacke, H.; Schröter, M.; Wagner, H. G. Symp. (Int.) Combust. [Proc.] 1979, 17, 505. mental studies of this important reaction.

We have used the recently developed Gaussian 2 method to characterize the stationary points on the $\mathrm{H}_{2} \mathrm{NNO}$ potential energy surface. Experimentally, we have used the laser-photolysis / cw LIF technique to measure the reaction-rate coefficient as a function of pressure, and temperature. We have also used the discharge-flow technique to characterize the reaction product branching fraction, as a function of temperature. In addition, we have investigated the effects of substituting $\mathrm{ND}_{2}$ for $\mathrm{NH}_{2}$ on both reaction rate coefficients and product branching fractions.

The integration of the results from these various experimental and theoretical studies is essential to gaining a full understanding of this complex bimolecular reaction. Energies of the stationary points on the potential energy surface for the reaction are shown in Figure 1. As expected, there are numerous "stable" intermediates separating reactants from products. Initial formation of an $\mathrm{H}_{2} \mathrm{NNO}$ adduct is followed by a 1-3 $\mathrm{H}$ shift leading to a $\mathrm{HNNOH}$ intermediate. This intermediate can undergo a cis-trans isomerization about the NO bond, a trans-cis isomerization about the $\mathrm{NN}$ bond and a four-center elimination to produce $\mathrm{N}_{2}+\mathrm{H}_{2} \mathrm{O}$ products. The $\mathrm{HNNOH}$ intermediates can also undergo bond fission to yield $\mathrm{N}_{2} \mathrm{H}+\mathrm{OH}$ products.

Experimentally, the reaction-rate coefficient is found to decrease with increasing temperature. This confirms the theoretical finding of a barrierless association of $\mathrm{NH}_{2}$ with NO. Association reactions typically exhibit pressure dependencies, but our calculated potential energy surface allows us to explain the lack of any experimentally observed pressure dependence; such a 


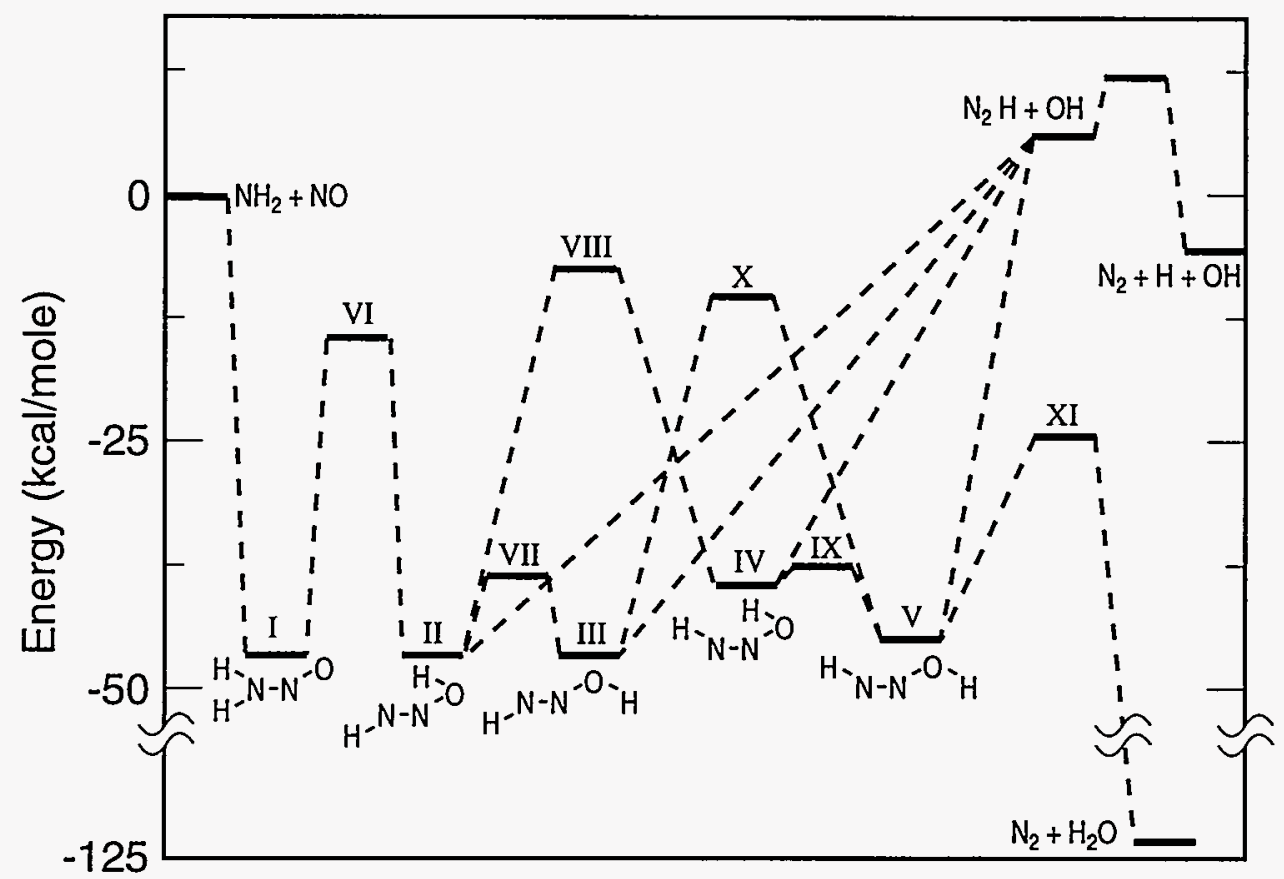

Figure 1: $\mathrm{H}_{2}$ NNO Potential Energy Surface.

pressure dependence arises from redissociation of the initially formed adduct, but in this case the initially formed $\mathrm{H}_{2} \mathrm{NNO}$ adduct has more than enough energy to rearrange to $\mathrm{HNNOH}$, and will not re-dissociate to $\mathrm{NH}_{2}+$ NO reactants.

The picture of a barrierless association reaction is also consistent with the effect of deuteration on the reaction-rate coefficient. Experimentally, we observe a temperature independent slowing of the reaction of $\mathrm{ND}_{2}$, relative to the reaction of $\mathrm{NH}_{2}$, by approximately $5 \%$. This is almost exactly the ratio of collision frequencies for $\mathrm{ND}_{2}+\mathrm{NO}$ and $\mathrm{NH}_{2}+\mathrm{NO}$.

Our studies of the product branching fraction in the $\mathrm{NH}_{2}+\mathrm{NO}$ reaction confirm that $\mathrm{N}_{2}+\mathrm{H}_{2} \mathrm{O}$ is the major product of the reaction. This is qualitatively what we would expect from an examination of the potential energy surface in Figure $1 . \mathrm{NH}_{2}+\mathrm{NO}$ reactants have sufficient energy to overcome all of the barriers separating them from $\mathrm{N}_{2}+$ $\mathrm{H}_{2} \mathrm{O}$ products. The $\mathrm{N}_{2} \mathrm{H}+\mathrm{OH}$ products are energetically above the reactants, and we expect their formation to be less favorable, especially at low temperatures.

We find that the $\mathrm{N}_{2} \mathrm{H}+\mathrm{OH}$ product channel is quite sensitive to deuterium substitution; at room temperature deuteration reduces it from $9 \%$ to $4.5 \%$ of the reactive flux. Preliminary calculations suggest that this is due to changes in the density of states at one of the transition states linking $\mathrm{HNNOH}$ intermediates. This observation highlights the importance of measuring isotope effects: these measurements give us additional insight into the detailed mechanism transforming reactants into products.

We are continuing our study of the reaction of $\mathrm{NH}_{2}$ with $\mathrm{NO}$. Areas of emphasis now include extension of our product branching fraction measurements to higher temperatures, and carrying out detailed calculations of the product branching fraction, reaction-rate coefficient and isotope effects. These calculations will be tested against our extensive experimental results. They then form the basis for a more accurate extrapolation of the behavior of this important reaction to conditions of temperature and pressure which are difficult to access experimentally.

This work was supported by the Division of Chemical Sciences, Office of Basic Energy Sciences, U.S. Department of Energy. 


\title{
Kinetic Investigations of the Reactions of $\mathrm{CH}$ [Х 2 with $\mathrm{CO}_{\text {and }} \mathrm{O}_{2}$
}

\author{
Precise measurements of reaction rates can be used to gain insight into the mechanisms of \\ critical combustion reactions. Using laser pump-probe experiments, we are investigating \\ reactions of many important radical intermediates, concentrating most recently on reactions \\ of the methylidyne $(\mathrm{CH})$ radical.
}

\section{A. Taatjes and R. T. Jennings}

The reactions of the methylidyne radical $(\mathrm{CH})$ are of fundamental interest in the understanding of combustion processes. Ground state $\mathrm{CH}\left(X^{2} \Pi\right)$ is extremely reactive, and because of its reactivity it is present in relatively low concentrations in flames. Nevertheless it is used extensively in flame diagnostics such as LIF imaging. It plays a role in a number of reactions important in hydrocarbon combustion. The reaction $\mathrm{CH}+$ $\mathrm{N}_{2} \rightarrow \mathrm{HCN}+\mathrm{N}$ is important in $\mathrm{NO}_{\mathrm{x}}$ production in combustion, and the $\mathrm{CH}+\mathrm{O}_{2}$ reaction is responsible for the $\mathrm{OH}\left(\mathrm{A}^{2} \Sigma\right)$ emission in flames. In addition, many $\mathrm{CH}$ radical reactions are becoming amenable to high-level calculations, making them attractive targets for detailed kinetics measurements.

The study of these reactions in our lab is carried out using the Laser Photolysis / cw Laser-Induced Fluorescence method (LP/cwLIF). The $\mathrm{CH}$ radicals are produced by multiphoton dissociation of $\mathrm{CHBr}_{3}$ by a $\mathrm{KrF}$ excimer laser, and the time evolution of the $\mathrm{CH}$ concentration is followed by LIF on the $A \leftarrow X$ transition at around $430 \mathrm{~nm}$. The probe laser is a linear dye laser with a linewidth of approximately $10 \mathrm{GHz}$, which is sufficient to resolve ${ }^{12} \mathrm{CH}$ from ${ }^{13} \mathrm{CH}$. In the $\mathrm{CH}+\mathrm{O}_{2}$ reaction we can also simply monitor the luminescence from the A-state $\mathrm{OH}$ product. The lasers are crossed in a heated stainlesssteel or quartz cell, and the interaction region is imaged onto a photomultiplier tube. Pressures between 10 and 760 Torr and temperatures from 250 to $1000 \mathrm{~K}$ are attainable. The present experiments were all carried out in a He buffer.
Our current studies on $\mathrm{CH}$ radical chemistry focus on the reactions

$$
\begin{aligned}
\mathrm{CH}+\mathrm{CO}(+\mathrm{M}) & \leftrightarrow \mathrm{HCCO}(+\mathrm{M}) \\
\mathrm{CH}+\mathrm{O}_{2}(+\mathrm{M}) & \rightarrow \mathrm{OH}+\mathrm{CO}(+\mathrm{M}) \\
& \rightarrow \mathrm{CO}_{2}+\mathrm{H}(+\mathrm{M}) \\
& \rightarrow \mathrm{HCO}+\mathrm{O}(+\mathrm{M})
\end{aligned}
$$

In the first reaction, $\mathrm{CH}+\mathrm{CO}$, the reactants constitute the lowest-energy bimolecular asymptote. Therefore the only reaction possible is association and stabilization to form HCCO. The ketenyl radical ( $\mathrm{HCCO}$ ) is itself an extremely important combustion species, produced in the reaction of acetylene with atomic oxygen. The $\mathrm{HOCO}$ and $\mathrm{HCOO}$ radicals are stable molecular configurations accessible in the $\mathrm{CH}+\mathrm{O}_{2}$ reaction. One intriguing aspect of the kinetic measurements is the possible relationship of these stable radicals to the reactive transition state.

In addition, it has been suggested that the $\mathrm{CH}+\mathrm{CO}$ reaction may access a symmetric oxiryl-like configuration, with the oxygen bound to both carbon atoms. A similar intermediate has been proposed for the ${ }^{1} \mathrm{CH}_{2}$ $+\mathrm{O}_{2}$ reaction. Evidence for a symmetric intermediate is the relative facility of carbon atom exchange, which we investigate using carbon-13 labeled reactants. The rates for the unlabeled reaction of $\mathrm{CH}+\mathrm{CO}$ are in reasonable agreement with previous determinations by other groups. The reaction exhibits a negative temperature dependence consistent with an association reaction, with a negative activation energy of $1.7 \mathrm{kcal} \mathrm{mol}^{-1}$. The pressure dependence of the reaction in $\mathrm{He}$ appears similar to the dependence observed 
by other groups in Ar buffer, although the reaction is slightly slower in $\mathrm{He}$.

The disappearance rate of ${ }^{12} \mathrm{CH}$ for the labeled reaction, ${ }^{12} \mathrm{CH}+{ }^{13} \mathrm{CO}$, is the sum of the association and the exchange component. Probing of the ${ }^{13} \mathrm{CH}$ produced in the reaction has been used as confirmation of the measured rates. The comparison of the labeled and unlabeled reactions reflects the competition between collisional stabilization and conversion to the presumed symmetric intermediate. While the exchange reaction is relatively fast, the rate is less than the highpressure limit for the reaction, i.e. the rate is not association limited. The question therefore arises whether the exchange reaction proceeds through the same initial collisional intermediate as the association reaction. A previous determination of the exchange reaction at low pressure was interpreted in connection with a common intermediate for association and exchange. The present results appear consistent with this interpretation, but additional measurements of the pressure and

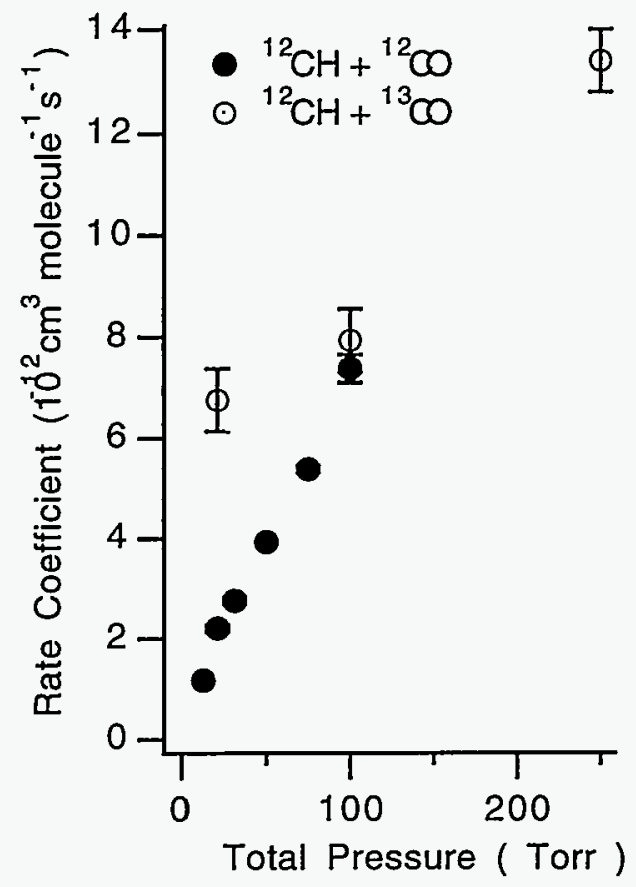

Figure 1. Reaction rates for $\mathrm{CH}+\mathrm{CO}$ as a function of total pressure. temperature dependence will provide a more detailed data for transition-state calculations.

The reaction of $\mathrm{CH}$ with $\mathrm{O}_{2}$ is in one sense

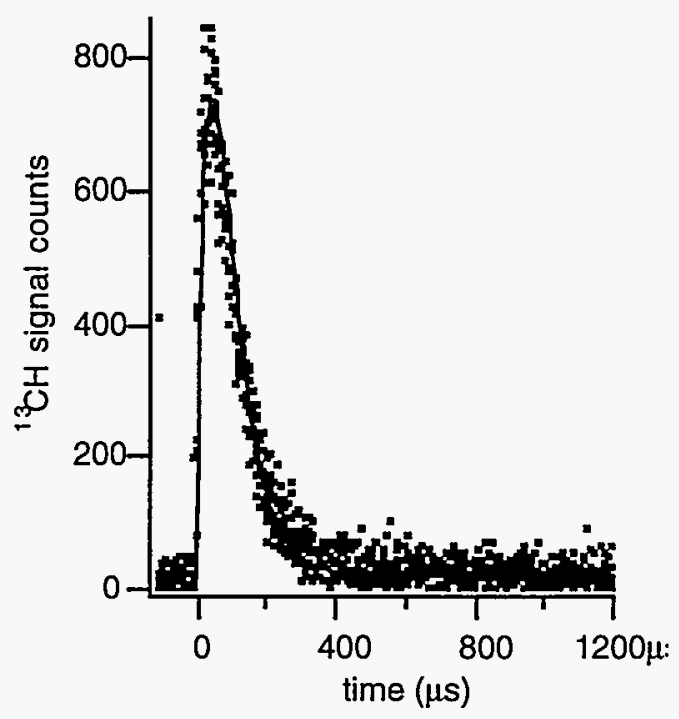

Figure $2 .{ }^{13} \mathrm{CH}$ signal from the reaction of ${ }^{12} \mathrm{CH}$ with ${ }^{13} \mathrm{CO}$. The solid line is a fit constructed from the measured ${ }^{12} \mathrm{CH}$ disappearance rates.

an opposite case to the $\mathrm{CH}+\mathrm{CO}$ reaction, since here we enter in the most, rather than the least, energetic bimolecular configuration. Extensive theoretical and experimental attention has been given the HOCO molecule, motivated by the important combustion reaction $\mathrm{OH}+\mathrm{CO} \rightarrow \mathrm{H}+\mathrm{CO}_{2}$. From the vantage point of $\mathrm{CH}+\mathrm{O}_{2}$, other configurations may become energetically accessible. For example, the $\mathrm{HCOO}$ radical is also a stable species which could play a role in the reaction. Our measured rate of $4.6 \pm 0.2 \times$ $10^{-11} \mathrm{~cm}^{3}$ molecule $\mathrm{e}^{-1} \mathrm{~s}^{-1}$ at room temperature in 100 Torr of He agrees well with previous measurements in an Ar buffer. However, preliminary results indicate a weak negative temperature dependence and a small positive pressure dependence at room temperature, with the rate at 250 torr $\mathrm{He}$ approximately 15 percent faster than at 100 Torr.

Further studies involving deuterium substitution, as well as detailed comparison to transition-state theory calculations, will offer a clearer picture of these two important combustion reactions. 


\title{
Thermal Decomposition of New Energetic Materials
}

\author{
TNAZ, 24DNI and NTO are being studied with the simultaneous
}

thermogravimetric modulated beam mass spectrometer (STMBMS) to determine

the reaction mechanisms that control their thermal decomposition. This data

will aid in developing models to predict the response of these materials to

external stimuli such as heat, impact and electrostatic discharge.

\section{R. Behrens, Jr., L. M. Minier, D. M. Puckett, and S. Bulusu 1}

Energetic materials (EM) are used in propellants, for systems such as rocket motors, guns and airbags, and in explosives for use in mining and munitions.

Although the desired properties and behavior of EMs are to some extent dependent on their applications, in general, it is desired that they possess a high energy content, high density, low sensitivity to heat, impact, shock and electrostatic discharge (ESD), and long term stability. Underlying an EMs sensitivity to heat, impact and ESD are the reaction mechanisms that control its thermal decomposition.

To design new energetic materials, it would be advantageous to know what role molecular structure, molecular functionality, crystal structure and physical properties play in the mechanisms that control the thermal decomposition process and hence, the sensitivity of these materials.

We study the thermal decomposition of EM using a simultaneous thermogravimetric modulated beam mass spectrometer (STMBMS) that has been described in detail.[1] Briefly, a $0.25 \mathrm{~cm}^{3}$ reaction cell containing $\sim 10 \mathrm{mg}$ of EM is heated under high vacuum. The gaseous thermal decomposition products evolve from the cell through a small orifice and the force change due to mass loss and thrust is measured with a microbalance and the gases are identified with a modulated beam mass spectrometer. Analysis of the resulting data provide the identities and rates of gas formation of the thermal decomposition products as a function of time.

${ }^{1}$ U.S. Army, ARDEC
From these results reaction mechanisms are derived as illustrated by previous work on HMX[2] and RDX.[3]

Several new EM are currently under investigation. These include 24DNI and NTO, which are thermally stable and relatively insensitive to impact, and TNAZ, which is a high performance material. Highlights of the results on 24DNI are presented to illustrate our work on these materials.<smiles>O=[N+]([O-])C1CC([N+](=O)[O-])([N+](=O)[O-])C1</smiles>

TNAZ<smiles>O=C1NNC([N+](=O)[O-])N1</smiles>

NTO<smiles>O=[N+]([O-])c1c[nH]c([N+](=O)[O-])n1</smiles>

24DNI

The thermal decomposition process in 24DNI occurs in both the solid and liquid phases. As can be seen in Figure 1, the gas formation rates are lower below the melting point $\left(272^{\circ} \mathrm{C}\right)$ and then increase rapidly as the sample melts. The ion signals shown in Fig. 1 are associated with the thermal decomposition products of 24DNI. These signals may be divided into three groups with different temporal behaviors that characterize the decomposition process. The first group includes $\mathrm{NO}_{2}$, and characterizes the first step in the decomposition process. The second group includes $\mathrm{NO}, \mathrm{H}_{2} \mathrm{O}, \mathrm{CO} / \mathrm{N}_{2}$, $\mathrm{NO}, \mathrm{HNCO}$, and $\mathrm{CO}_{2}$, the gaseous products 


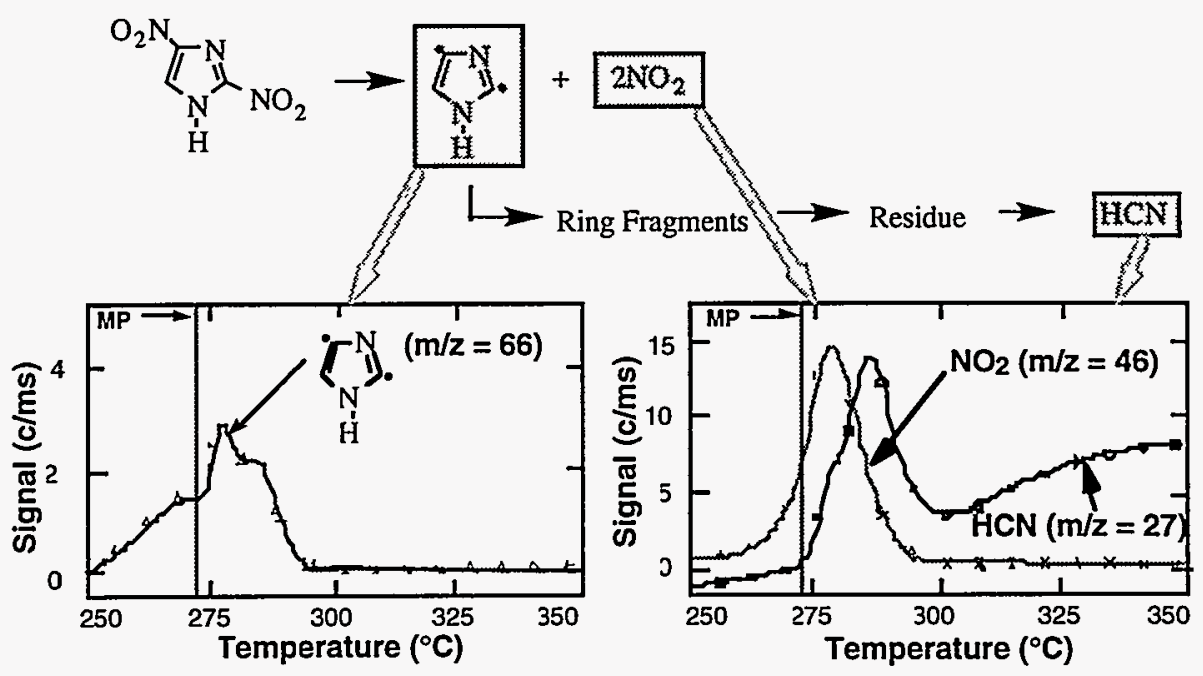

Figure 1. Sequenced of product release from the decomposition of 24DNI.

evolved from the reaction. The third group includes $\mathrm{HCN}$ and $\mathrm{C}_{2} \mathrm{~N}_{2}$, which come from the decomposition of a polymeric material that was formed as the gases associated with the second group were released.

One of the reaction pathways that control this process is summarized in Figure 1. First, the 24DNI undergoes $\mathrm{C}-\mathrm{NO}_{2}$ bond breaking. Next, the $\mathrm{NO}_{2}$ group leaves and
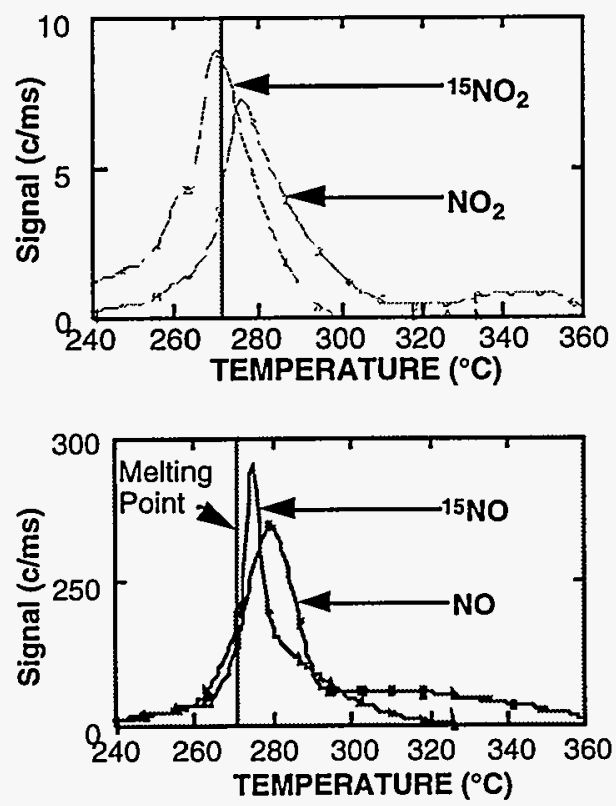

Figure 2. Evolution of $\mathrm{NO}_{2}$ and $\mathrm{NO}$ from 24DNI labeled with ${ }^{15} \mathrm{NO}_{2}$ at the 2-position. the resulting radical undergoes polymerization. Finally, the polymer decomposes producing $\mathrm{HCN}$ and $\mathrm{C}_{2} \mathrm{~N}_{2}$ products.

The decomposition process is examined in further detail using 24DNI with ${ }^{15} \mathrm{NO}_{2}$ in the 2-position. As can be seen from the results in Figure 2, the $\mathrm{NO}_{2}$ and $\mathrm{NO}$ evolved first from the 2-position in the molecule forming a reaction intermediate that then undergoes slower reaction releasing $\mathrm{NO}_{2}$ and $\mathrm{NO}$ from the 4-position.

STMBMS experiments on new energetic materials are providing new insight and extensive details on the reaction pathways that control their thermal decomposition processes. These mechanisms will be used in new models to predict the behavior of these materials in abnormal environments.

\section{References}

[1] a) R. Behrens, Rev. Sci. Instrum., 58, 451461 (1987); R. Behrens, b) Int. J. Chem, Kinetics, 22, 135-157,(1990); c) R. Behrens, Int. J. Chem. Kinetics, 22, 159173,(1990).

[2] a) R. Behrens, J. Phys. Chem., 94, 6706 6718 (1990); b) R. Behrens and S. Bulusu, J. Phys. Chem., 95, 5838 - 5845, (1991).

[3] a) R. Behrens and S. Bulusu, J. Phys. Chem, 96, 8877-8891, (1992); b) R. Behrens and S. Bulusu, J. Phys. Chem, 96, 8891-8897, (1992). 


\title{
The Oxidation of Allene in Flames
}

\author{
In order to understand better the chemistry accompanying benzene formation in flames of \\ aliphatic fuels, we have investigated a series of flames in which allene is oxidized. \\ Considerable insight has been obtained into the combustion chemistry of $\mathrm{C}_{3} \mathrm{H}_{3}$ and $\mathrm{C}_{3} \mathrm{H}_{2}$ \\ free radicals. Our results are summarized below.
}

\section{J. A. Miller, J. V. Volponi, and J.-F. Pauwels 1}

Work in several laboratories has recently focused attention on the possibility that the propargyl radical, $\mathrm{C}_{3} \mathrm{H}_{3}$, is instrumental in forming the "first ring" in flames of aliphatic fuels. This first ring ultimately leads to the formation of polycyclic aromatic hydrocarbons (PAH) and soot. Work in our laboratory [1] has shown that reactions of $\mathrm{C}_{4} \mathrm{H}_{3}$ and $\mathrm{C}_{4} \mathrm{H}_{5}$ isomers with acetylene are inadequate to account for the quantities of benzene $\left(\mathrm{C}_{6} \mathrm{H}_{6}\right)$ observed in lightly sooting acetylene flames. However, propargyl, which is a resonantly stabilized free radical, can build up to fairly high concentrations in flames and react with itself (or perhaps with $\mathrm{C}_{3} \mathrm{H}_{2}$ ) to form $\mathrm{C}_{6} \mathrm{H}_{5}$ (phenyl) $+\mathrm{H}$ (or $\mathrm{C}_{6} \mathrm{H}_{6}$ ), thus producing the first six-membered ring. Electronic-structure calculations [1,2] show several paths with no intrinsic energy barriers that lead from two propargyl radicals to $\mathrm{C}_{6} \mathrm{H}_{5}$ $+\mathrm{H}$ or $\mathrm{C}_{6} \mathrm{H}_{6}$.

In pursuing these other investigations, we came to realize that relatively little is known about how propargyl and related species (allene, methylacetylene and various $\mathrm{C}_{3} \mathrm{H}_{2}$ isomers) react in flames. In order to understand this chemistry better we initiated three separate flame studies. In the first investigation, we added a small amount of allene $\left(\mathrm{C}_{3} \mathrm{H}_{4}\right)$ to a well-characterized rich $(\varnothing=$ 1.5) $\mathrm{H}_{2} / \mathrm{O}_{2} /$ Ar flame. This situation allowed us to study the oxidation of allene under conditions that are similar to those encountered in rich hydrocarbon flames, but without having to account for complications due to reactions between hydrocarbon species. In the second investigation we studied a rich $(\varnothing=1.67) \mathrm{C}_{2} \mathrm{H}_{2} / \mathrm{O}_{2} / \mathrm{Ar}$ flame with and without allene addition, and in work ongoing

$\overline{1}$ Universite' des Sciences et Techniques de Lille, France in our laboratory we are studying two flames, one rich and one lean, in which allene is the only fuel.

In all the flames studied, stable gas species were extracted by a quartz microprobe and measured quantitatively with a mass spectrometer. Radical gas species $(\mathrm{CH}$ and $\mathrm{OH})$ and temperatures were determined by laser-induced fluorescence from $\mathrm{OH}$ using an excimer-pumped dye laser system. The flames were stabilized on a water-cooled flat-flame burner fabricated from sintered stainless steel. The burner is housed in a vacuum chamber maintained at 25 Torr. Species profiles were obtained by vertically translating the burner relative to the probe or laser beam (position accuracy $\pm 0.02 \mathrm{~cm}$ ).

In addition to the $\mathrm{OH}$ rotational temperature measurements (obtained from LIF), we found it necessary to make temperature measurements using thermocouples. Platinum/platinum - $13 \%$ rhodium thermocouples, coated to reduce catalytic effects, were used to measure temperatures both with and without the sampling probe in place. Thermocouple temperatures and LIF temperatures without the probe present were generally in good agreement. Temperatures obtained with the probe in the flame were somewhat lower. Particularly large differences were found for the $\mathrm{C}_{2} \mathrm{H}_{2} / \mathrm{O}_{2} / \mathrm{Ar}-\mathrm{C}_{3} \mathrm{H}_{4}$ flame. Consequently, in modeling the flames we used temperature profiles obtained with the probe present to make predictions of stablespecies concentrations and temperature profiles obtained with the probe removed to make predictions of $\mathrm{OH}$ and $\mathrm{CH}$ concentrations. The differences in predictions were really significant only for the acetyleneallene flame. 
In the $\mathrm{H}_{2} / \mathrm{O}_{2} / \mathrm{Ar}-\mathrm{C}_{3} \mathrm{H}_{4}$ flame (one percent allene) we somewhat unexpectedly found significant quantities of methane and acetylene early in the flame. However, the analysis (flame modeling) showed that these species were formed as a consequence of recombination of propargyl with hydrogen atoms; i.e.,

$$
\begin{aligned}
& \mathrm{C}_{3} \mathrm{H}_{4}+\mathrm{H} \leftrightarrow \mathrm{C}_{3} \mathrm{H}_{3}+\mathrm{H}_{2} \\
& \mathrm{C}_{3} \mathrm{H}_{3}+\mathrm{H}(+\mathrm{M}) \leftrightarrow \mathrm{C}_{3} \mathrm{H}_{4} \mathrm{P}(+\mathrm{M}) \\
& \mathrm{C}_{3} \mathrm{H}_{4} \mathrm{P}+\mathrm{H} \leftrightarrow \mathrm{C}_{2} \mathrm{H}_{2}+\mathrm{CH}_{3} \\
& \mathrm{CH}_{3}+\mathrm{H}(+\mathrm{M}) \leftrightarrow \mathrm{CH}_{4}(+\mathrm{M}),
\end{aligned}
$$

where $\mathrm{C}_{3} \mathrm{H}_{4} \mathrm{P}$ is methylacetylene (propyne). Because $\mathrm{C}_{3} \mathrm{H}_{3}$ is so stable (resonance stabilization) it does not react very rapidly with molecular oxygen. Consequently, propargyl reacts primarily with hydrogen atoms. The principal path is

$$
\mathrm{C}_{3} \mathrm{H}_{3}+\mathrm{H} \leftrightarrow \mathrm{C}_{3} \mathrm{H}_{2}+\mathrm{H}_{2}
$$

with reaction (2) and its counterpart to form allene representing important minor paths. Allene oxidation occurs primarily through the step

$$
\mathrm{C}_{3} \mathrm{H}_{2}+\mathrm{O}_{2} \leftrightarrow \mathrm{HCCO}+\mathrm{CO}+\mathrm{H} .
$$

Results in both the hydrogen-allene and acetylene-allene flames are sensitive to the rate coefficient and products of reaction (6). The products shown are analogous to those of the similar reaction between triplet methylene and $\mathrm{O}_{2}$,

$$
{ }^{3} \mathrm{CH}_{2}+\mathrm{O}_{2} \leftrightarrow \mathrm{CO}+\mathrm{OH}+\mathrm{H} .
$$

In all our modeling $\mathrm{C}_{3} \mathrm{H}_{2}$ was assumed to be triplet progargylene. However, there are several other isomers of $\mathrm{C}_{3} \mathrm{H}_{2}$ that could participate in the chemistry.

The most important result of our investigation of the effects of adding allene to the $\mathrm{C}_{2} \mathrm{H}_{2} / \mathrm{O}_{2} /$ Ar flame was the appearance of benzene in the flame when allene was added. This observation supports the contention that the reaction,

$$
\mathrm{C}_{3} \mathrm{H}_{3}+\mathrm{C}_{3} \mathrm{H}_{3} \leftrightarrow \mathrm{C}_{6} \mathrm{H}_{5}+\mathrm{H},
$$

is a primary cyclization step in flames. In addition to the rate coefficient for reaction (7) the predicted benzene profile was also sensitive to the rate coefficients for the reactions,

$$
\begin{aligned}
& \mathrm{C}_{6} \mathrm{H}_{5}+\mathrm{H} \leftrightarrow \mathrm{C}_{6} \mathrm{H}_{6} \\
& \mathrm{C}_{6} \mathrm{H}_{5}+\mathrm{O}_{2} \leftrightarrow \mathrm{C}_{6} \mathrm{H}_{5} \mathrm{O}+\mathrm{O} .
\end{aligned}
$$

which compete with each other for phenyl. The recent determination of the rate coefficient for reaction (9) by Frank and co-workers [3] made quantitative prediction of our benzene profiles possible.

\section{References}

[1] J. A. Miller and C. F. Melius, Combust. Flame 91, 21 (1992)

[2] C. F. Melius, J. A. Miller, and E. Y. Evleth, Twenty-Fourth Symposium (International) on Combustion, The Combustion Institute, Pittsburgh, PA, p. 621 (1992)

(3) P. Frank, J. Herzler, Th. Just, and C. Wahl, Twenty-Fifth Symposium (International) on Combustion, The Combustion Institute, Pittsburgh, PA (1995), in press 


\section{Theoretical Predictions of Fullerene Hydride Chemistry}

Simple hydrocarbon derivatives of the most abundant fullerenes, $C_{60}$ and $C_{70}$, are characterized by their geometry and energetics using ab initio quantum chemical theory. Subsequent experimental syntheses confirm the accuracy of the theoretical predictions.

\section{M. Rohlfing, P. A. Cahill, and C. C. Henderson}

Macroscopic quantities of the spheroidal fullerenes $\mathrm{C}_{60}$ and $\mathrm{C}_{70}$ were first produced in 1990 by graphite vaporization with resistive heating in an inert gas. Subsequently these fullerenes and their simple derivatives have been detected in sooting flames, meteor fragments, and interstellar dust. The development of new fullerene-based materials and compounds is now an intensive research area.

Chemical reactions involving $\mathrm{C}_{60}$ and $\mathrm{C}_{70}$ often produce complex mixtures of products. Even after separation, standard identification methods such as ${ }^{13} \mathrm{C}$ NMR cannot always distinguish between the various isolated isomers. Rigorous theoretical analysis of the geometries and energetics of the possible products using ab initio quantum chemical methods provides the missing information which is key to the final structure determinations.

The simple hydrocarbon fullerene derivatives $\mathrm{C}_{60} \mathrm{H}_{2}$ and $\mathrm{C}_{70} \mathrm{H}_{2}$ typify the regiochemistry of $X_{2}$ addition to fullerenes. For $\mathrm{C}_{60} \mathrm{H}_{2}$, there are 23 structurally distinct isomers. The lowest energy form predicted by both semiempirical and ab initio quantum chemical calculations is $1,2-\mathrm{C}_{60} \mathrm{H}_{2}$, which corresponds to 1,2-addition to a 6:6-ring fusion [1]. This is not an unexpected result since the short (double) bond at the 6:6-ring fusion in $\mathrm{C}_{60}$ should be more stable upon reduction than the long (single) bond at the 5:6-ring fusion. The only isomer observed experimentally [2] is the same as the calculated lowest energy structure, and it appears to be both the kinetic and thermodynamic product of the hydrogenation reaction.
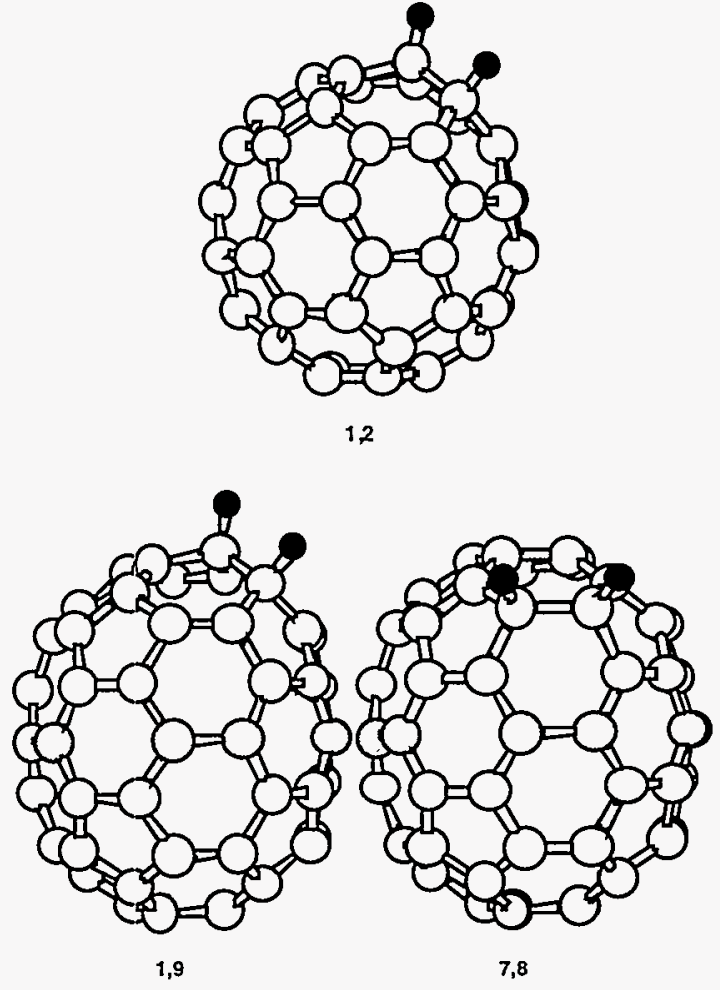

Figure 1. Lowest energy isomers of $\mathrm{C}_{60} \mathrm{H}_{2}$ and $\mathrm{C}_{70} \mathrm{H}_{2}$.

For $\mathrm{C}_{70} \mathrm{H}_{2}$, there are 143 structurally distinct isomers. Semiempirical methods were employed first to screen out high-energy structures, and then complete geometry optimizations of low-energy isomers were performed at the Hartree-Fock (HF) level using the $6-31 \mathrm{G}^{*}$ basis set [1]. Hydrogen addition follows the general results seen for $\mathrm{C}_{60}$, in that it is most favored to occur 1,2 to 6:6-ring fusions. 
At the ab initio level, $1,9-\mathrm{C}_{70} \mathrm{H}_{2}$ (which corresponds to the lowest energy $1,2-\mathrm{C}_{60} \mathrm{H}_{2}$ isomer) is slightly favored by $1.3 \mathrm{kcal} / \mathrm{mol}$ over $7,8-\mathrm{C}_{70} \mathrm{H}_{2}$ (a structure not possible for $\mathrm{C}_{60} \mathrm{H}_{2}$ ). Semiempirical calculations reverse this ordering. However, only the predictions at the ab initio level are consistent with experiment -.. the 1,9 isomer is the thermodynamically most stable isomer in the synthesis of $\mathrm{C}_{70} \mathrm{H}_{2}$ [3], with one other minor product being the 7,8 isomer. Equilibration measurements put $7,8-\mathrm{C}_{70} \mathrm{H}_{2}$ at $1.4 \mathrm{kcal} / \mathrm{mol}$ above $1,9-\mathrm{C}_{70} \mathrm{H}_{2}$, in excellent agreement with the $a b$ initio results.

The regiochemistry of multiple $X_{2}$ addition to $\mathrm{C}_{60}$ was investigated for the case of $\mathrm{C}_{60} \mathrm{H}_{4}$ [4]. Eight isomers can result from 1,2 addition to 6:6-ring fusions. At the ab initio level, isomer 1 (in which the four hydrogens have added to adjacent 6:6-ring fusions) is favored by $4.0 \mathrm{kcal} / \mathrm{mol}$ over isomer 4 . In contrast, semiempirical calculations predict 1 to be a high energy structure. Once again, only the findings at the $a b$ initio level are consistent with the subsequent experiment. Synthesis of $\mathrm{C}_{60} \mathrm{H}_{4}$ [4] produces multiple isomers, which upon isolation, characterization and equilibration yield isomer 1 as the major thermodynamic and kinetic product of multiple hydrogenation.

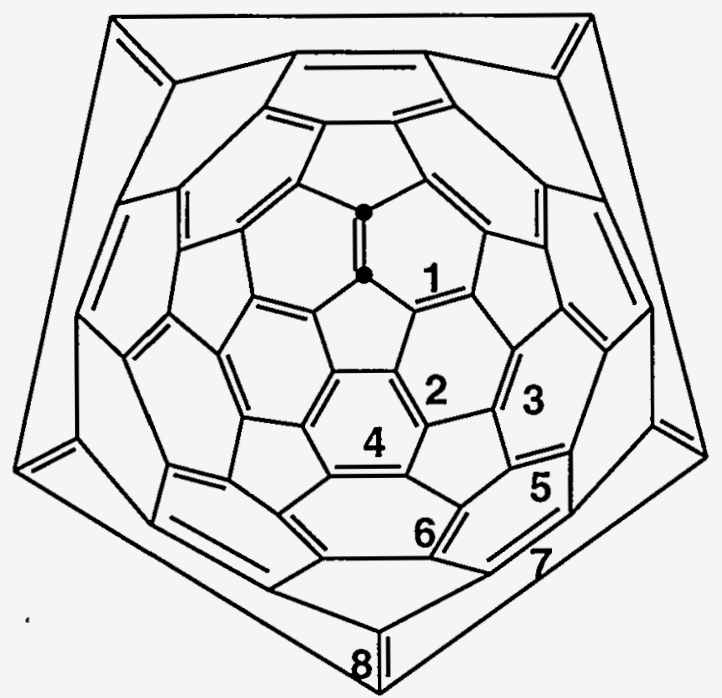

Figure 2. Nomenclature for eight isomers of $\mathrm{C}_{60} \mathrm{H}_{4}$.
This work was supported by Sandia 's Laboratory Directed Research and Development Program.

\section{References}

[1] Henderson, C. C., Rohlfing, C. M., Cahill, P. A. Chemical Physics Letters 213:383, 1993.

[2] Henderson, C. C., Cahill, P. A. Science 259:1885, 1993.

[3] Henderson, C. C., Rohlfing, C. M., Gillen, K. T., Cahill, P. A. Science 264:397, 1994.

[4] Henderson, C. C., Rohlfing, C. M., Assink, R. A., Cahill, P. A. Angewandte Chemie 33:786, 1994. 


\section{Studies in Combustion Dynamics}

The goal of this program is to develop a fundamental understanding and a quantitative predictive capability in combustion modeling. Modeling involves the integration of methods from chemistry, fluid mechanics and computational science. Recently we developed new

tools for parallel processing that enable modeling of a turbulent diffusion flame model including a "full" chemical mechanism.

\section{L. Koszykowski, R. C. Armstrong and J.-Y. Chen}

Presently, one of the most important problems in turbulent combustion modeling is correctly approximating the coupling between reactive and diffusive processes on the smallest scales. Future progress in combustor design is very promising if modeling capabilities are further extended so that detail at a finer level of structure can be predicted. To be sufficiently accurate to be used as design tools, these models and their corresponding computational codes must include both chemistry, fluid mechanics, and their interactions over a broad range of time and length scales.

While fluid-mechanical turbulence models and detailed-chemistry flame models in simple flows are solvable on standard vector supercomputers, the combination of turbulent flow and detailed chemistry in the same model requires the next generation supercomputer: the massively parallel machine. We have investigated a probability density function (PDF) code for a jet flame diffusion problem. The PDF algorithm involves mostly Monte Carlo calculations and is highly amenable to an efficient parallel implementation. A toolkit approach is used to partition the algorithmic portions of the code (e.g. equation solver, Monte Carlo simulation) from the application specific code.

Existing tools for parallel software development generally fall into two categories: 1) high-level tools and compilers that hide the parallelization details, making them easy to use but also hiding the pitfalls that lead to bottlenecks; or, 2) low-level tools for message passing that create scalable code, but require detailed knowledge of algorithms and software that are difficult for the non-systems programmer to use. The purpose of our approach, that of a toolkit, is to allow a physical scientist to create an integrated and scaiable application code that transparently accesses parallel computing resources and avoids the traditional pitfalls associated with parallel computing.

We have investigated a turbulent diffusion flame that consists of a cylindrical jet which injects a fuel into a coflowing air stream and is ignited. The model is composed of three parts: the turbulent motion model, the chemical reaction model and the coupling between chemical reactions and turbulence. The downstream exhaust is computed using an advancing grid beginning from the inlet and advancing downstream until chemical activity is complete. Symmetry allows this problem to be modeled using a one dimensional grid that effectively represents a radial slice of the cylindrical nozzle.

The algorithm is a piecewise Monte Carlo method in which the Monte-Carlo aspect of the problem comes from the transport (or mixing) of particles to and from nearest-neighbor cells. This mixing is a result of fluid-mechanical turbulence and Fickian diffusion. The chemistry that accounts for most of the computation, is local to each cell in which particles from one node cell are required to communicate with only the right and left neighbor cells. 
The entire one-dimensional grid of cells plus boundary conditions for the two cells on left and right ends of the grid compose the Monte Carlo portion of the problem and accounts for $99.3 \%$ of the computer time, even with minimal chemistry. At the appropriate time, statistics computed from the particles distributed over the cells contribute to an update of the flow field, either as an iteration converging on a steady state or as a time step in a nonsteady problem. The addition of realistic chemistry increases the computation required by 10 to 100 fold.

Through our toolkit, the power necessary to compute PDF chemically reacting flow problems with realistic chemistry on massively parallel and distributed systems has been provided. Moreover, by encapsulating the PMC algorithm in an autonomous object and thus insulating the specific physics of a PDF problem from the algorithm, we produce reusable code that can be used on other chemically reacting flow problems.

Concentration and temperature profiles for three flames has been computed with a full chemical mechanism as well as a reduced mechanism In all cases the hydrogen fuel jet was composed of $40 \% \mathrm{He}$. The input conditions were chosen to simulate the experiments. All calculations were completed using two different mechanisms. Mechanism one was reduced from the detailed mechanism. The second mechanism used was a comprehensive mechanism. The two chemical mechanisms yield temperature profiles that are very similar, although the full mechanism yields contours that are $5 \%$ wider. Examination of the actual numerical values of temperature at the various grid points reveals that peak temperatures are equal to within 10 degrees. Calculated NO profiles generated from comprehensive and reduced chemistry show that the detailed mechanism predicts substantially more NO formation. The maximum NO concentration is approximately a factor of two larger with the comprehensive mechanism. To further explore the reason for the difference, it is important to examine the $\mathrm{O}$ atom profile. The $\mathrm{O}$ atom concentration is substantially larger and persists over a larger distance when the comprehensive mechanism is used, showing the assumption of partial equilibrium among radical species, implicit in the reduced mechanism, is not valid.

The NOx emission index was calculated for each chemical mechanism as a function of Reynolds number. As the Reynolds number increases NO decreases because the residence time decreases. The detailed mechanism predicts an emission index that is in excellent agreement with experiment, while the reduced mechanism significantly under predicts the NO formation as shown in Figure 1.

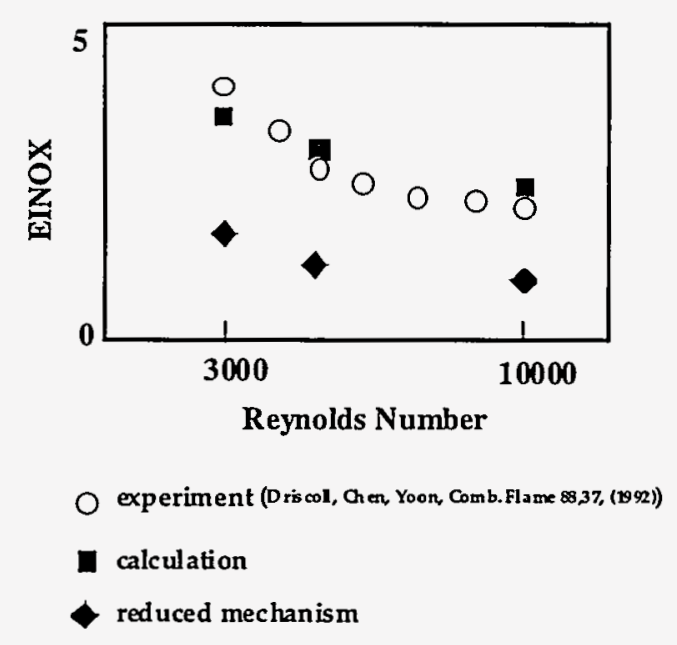

Figure 1: Emission index using full chemistry shows significantly improved results.

Current research is focused on extending the fluid mechanical model used to a time dependent LES model. We have also begun calculations of methane flames using the present model.

This work was supported by the Division of Chemical Sciences, Office of Basic Energy Sciences, U.S. Department of Energy. 



\title{
Reacting Flows
}

\author{
Introduction
}

Research on fundamental aspects of turbulent reacting flows is central to the mission of the Combustion Research Facility (CRF). These projects include experimental, numerical, and analytical approaches by an interdisciplinary team of researchers. Maximal impact on this difficult and complex field is sought by taking advantage of advanced laser-based diagnostics, unique experimental capabilities, state-of-the-art computing, and strong collaborations with the international combustion community. An important theme of this work has been to further our understanding of the coupling between turbulent fluid dynamics and combustion chemistry.

Experimental reacting flow research employs laser diagnostics for both singlepoint multi-species measurements and twodimensional spatial imaging of one to three molecular species. Raman, Rayleigh, Mie, and laser-induced fluorescence techniques allow non-intrusive measurements of combustion radicals, major species, temperature, and velocity profiles in specialized combustion environments. Comparisons of the data with model predictions are providing researchers at the $\mathrm{CRF}$ and elsewhere new insights into turbulent combustion phenomena and improved combustion technology.

Numerical and analytical projects focus on regimes where there is consequential coupling of fluid mechanical and chemical effects. Pollutant formation, flame stability, and turbulent flame speeds are examples of important issues that are addressed by several complementary approaches. Direct numerical simulations allow investigations that include chemical effects with few assumptions concerning the fluid dynamics, but are still limited to small volumes by computer capacity. Statistical and analytical methods allow consideration of issues important at larger scales and often benefit from insight gained through numerical simulations as well as experimental measurements.

Reacting flow research at the CRF benefits from frequent long-term visitors from around the world who come to use unique facilities and work with the resident principal investigators. The major portion of this work as well as user facility operations and the visitor program are funded by the Chemical Sciences Division of the Office of Basic Energy Sciences, United States Department of Energy. 


\title{
Measurements of Nitric Oxide Formation in Turbulent Hydrogen Jet Flames
}

\author{
The relationships among nitric oxide concentration, temperature, and mixture fraction have \\ been investigated experimentally. These relationships are strongly influenced by the \\ streamwise evolution of turbulent reaction-zone structure in the jet flame.
}

\section{R. S. Barlow, C. D. Carter 1 , and T. L. Prast}

One of the critical objectives of current research and development efforts in combustion is to attain the capability to predict and minimize $\mathrm{NO}_{\mathrm{x}}$ formation in practical combustion devices. A detailed knowledge of NO formation mechanisms in simple turbulent jet flames is an important step toward this goal. Previous experimental studies of $\mathrm{NO}_{\mathbf{X}}$ formation in jet flames have relied primarily on gas-sampling probe techniques, which yield only timeaveraged data. The present experiments were conducted to determine the instantaneous relationships between NO concentration and other scalars and to illuminate the role of turbulence-chemistry interactions in $\mathrm{NO}_{\mathrm{X}}$ formation.

Spontaneous Raman scattering, Rayleigh scattering, and laser-induced fluorescence were combined to obtain simultaneous point measurements of $\mathrm{NO}$, the major species, $\mathrm{OH}$, and temperature in turbulent hydrogen jet flames [1-3]. To provide a detailed basis for evaluation of combustion models, extensive measurements were made at several streamwise locations in an undiluted hydrogen jet flame with a jet Reynolds number of 10,000 and in two helium diluted hydrogen flames. Dilution with helium reduces the radiative fraction to low levels, allowing for independent evaluation of turbulence-chemistry submodels and radiation submodels.

Scatter plots (Fig. 1) reveal the instantaneous relationship between NO and mixture fraction in the undiluted $\mathrm{H}_{2}$ jet flame. The highest NO concentrations occur near the stoichiometric condition, where temperatures are also highest. There is a significant increase in NO levels between the two streamwise sta-

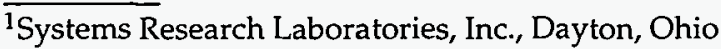
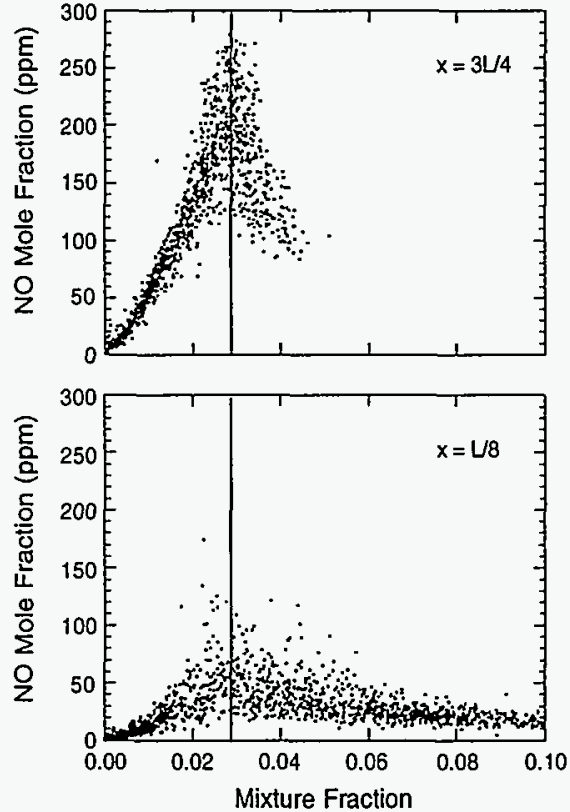

Figure 1. Scatter plots of NO mole fraction at two streamwise locations relative to the visible flame length, $L$. The mixture fraction is zero in air and unity in pure fuel. The stoichiometric value of 0.0285 for undiluted $\mathrm{H}_{2}$ fuel is indicated by the vertical line.

tions included in Fig. 1. However, the relative fluctuations near the stoichiometric mixture fraction decrease from 50 percent of the local mean at $x=L / 8$ to 20 percent at $x=3 \mathrm{~L} / 4$. This can be attributed to a streamwise evolution of the reaction zone structure.

Near the nozzle, NO is formed in thin reaction zones that have some of the characteristics of strained laminar flames. NO production rates in these thin zones are sensitive to local turbulent strain, due to the effects of strain on temperature depression and O-atom superequilibrium. NO concentrations are 


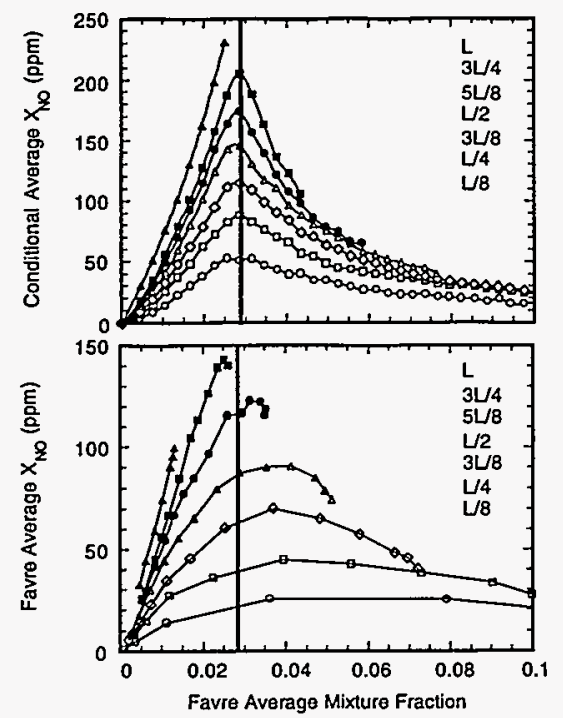

Figure 2. Conditionally averaged and Favre averaged results for $N O$ mole fraction $X_{N O}$ vs. mixture fraction at seven streamwise locations (indicated in order by inset) in an undiluted $\mathrm{H} 2$ jet flame.

doubly sensitive to local scalar dissipation rates because of the combined effects of nonequilibrium chemistry and residence time. High scalar dissipation means low temperature (relative to equilibrium) and short residence time at the NO forming conditions. Farther downstream $(x=3 \mathrm{~L} / 4)$ scalar dissipation decreases, radical concentrations approach equilibrium, the high-temperature zones become broad, and NO concentrations at a given mixture fraction become more uniform.

Fig. 2 examines the averaged relationship between NO and mixture fraction and shows that conditional averaging and Favre (density weighted) averaging give very different results. With the former, NO concentrations are averaged conditionally on the value of the simultaneously measured mixture fraction. All data from a full radial profile are used to generate the conditional average curve for a given streamwise station. These curves show a gradual streamwise increase in NO, with the peak levels consistently occurring near the stoichiometric condition. The Favre averages are analogous to gas-sampling probe results, with each point on the graph corresponding to a single spatial location in the flame. The Favre averaged curves show a bias or "rich shift" in the relationship between $X_{\mathrm{NO}}$ and mixture

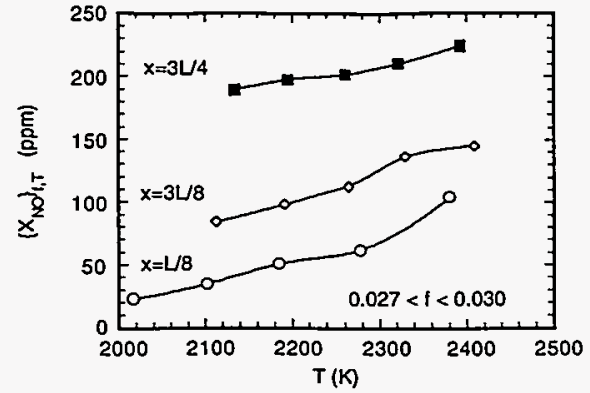

Figure 3. Doubly conditional averages of NO mole fraction at three loacations in the $\mathrm{H}_{2}$ jet flame.

fraction. The largest bias occurs at $\mathrm{x}=\mathrm{L} / 8$, where density fluctuations are greatest. This bias is inherent to the Favre averaging process and should be considered whenever measurements are made within turbulent flames.

The relationship between NO concentration and temperature can be quantified by conditioning on both mixture fraction and temperature (Fig. 3). Here only near-stoichiometric samples are considered, and data are divided into temperature bins before averaging. At $\mathrm{x}=\mathrm{L} / 8$ the doubly conditional $\mathrm{NO}$ mole fraction, $\left\{X_{N O}\right\}_{f}, T$, increases by a factor of three over the temperature range from $2100 \mathrm{~K}$ to $2400 \mathrm{~K}$. At $\mathrm{x}=3 \mathrm{~L} / 4$ there is only a 20 percent increase in NO over the same temperature range.

Accurate prediction of these experimental results is providing a significant test for turbulent combustion models.

This work supported by the Division of Chemical Sciences, Office of Basic Energy Sciences, U.S. Department of Energy.

\section{References}

[1] C.D. Carter and R.S. Barlow, Optics Letters, 19, 229-301, (1994).

[2] R.S. Barlow and C.D. Carter., "Relation ships Among Nitric Oxcide, Temperature and Mixture Fraction in Hydrogen Jet Flames." submitted for publication in Combustion and Flame.(1994). 


\title{
Temporal Evolution of Turbulence/Chemistry Interactions Studied in Nonpremixed Flames
}

\author{
A double-pulse, planar imaging technique was used to image the instantaneous $\mathrm{CH}$ and $\mathrm{CH}_{4}$ \\ distributions in turbulent, lifted-jet flames. The double pulses provide unique insights into \\ the time development of interactions between turbulence and flame chemistry that lead to \\ local ignition, extinction, and reignition in turbulent flames.
}

\section{R. W. Schefer, M. Namazian, ${ }^{1}$ and J. Kelly 1}

Flame stabilization is an issue of fundamental importance to turbulent combustor design. This is especially true inthe design of low $\mathrm{NO}_{x}$ burners where flame stability is often adversely affected by $\mathrm{NO}_{x}$ reduction strategies. Flame stabilization theories generally recognize the importance of local ignition, flame propagation, extinction and reignition phenomena as factors controlling flame stability[1]. These theories are largely based on experimental observations in turbulent, lifted-jet flames where many of these phenomena are present. An understanding of the stabilization mechanisms for lifted flames is thus key to a better understanding of flame stabilization in general.

Since flame ignition, extinction and propagation are inherently time-dependent phenomena, time-resolved measurements are required to understand their role in flame stabilization. Recognizing the importance of such measurements, we have obtained twopulse images of the $\mathrm{CH}$ and $\mathrm{CH}_{4}$ distributions in a turbulent, lifted $\mathrm{CH}_{4}$-jet flame. The experiment was designed to provide two laser pulses with a predefined, variable time delay between pulses so that a range of transient events having different time scales can be studied. The two-camera imaging system used here is similar to that used in previous singleshot $\mathrm{CH} / \mathrm{CH}_{4}$ studies[2]. Two temporallyseparated pulses were provided by the Combustion Research Facility's flashlamppumped dye laser, which was reconfigured into two separate lasers consisting of one oscillator and amplifier stage each. The orthogonally-polarized beams were combined using a polarizing beamsplitter and formed

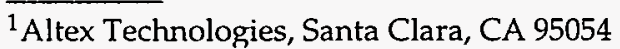

into an overlapping sheet in a multipass cell. The beams were tuned to a wavelength of $431.5-\mathrm{nm}$, which was used to excite the $(0,0)$ band of the $A^{2} \Delta \leftarrow X^{2} I I$ system of $C H$, and fluorescence from the $(0,1)$ transition was observed at $489 \mathrm{~nm}$. Simultaneously, spontaneous Raman scattering from $\mathrm{CH}_{4}$ also occurred near $489 \mathrm{~nm}$ with a $431.5-\mathrm{nm}$ excitation. The combined $\mathrm{CH}$ fluorescence and $\mathrm{CH}_{4}$ Raman-scattering signals were collected at a right angle to the laser sheet by $\mathrm{f} / 1.2$ camera lenses and detected at $489 \mathrm{~nm}$ using two intensified CCD cameras located on opposite sides of the laser sheet. Timing pulse generators were used to fire both lasers with a variable time delay and to gate both camera intensifiers so that the first laser shot was recorded on one camera and the second shot on the other camera.

The burner consisted of a 5.4- $\mathrm{mm}$ diameter fuel jet located in the center of a plate. Methane was injected through a central fuel tube into surrounding still air at velocities of $21 \mathrm{~m} / \mathrm{s}$, corresponding to a fuel-jet Reynolds number of 7,000 . At this flow condition, the visible flame was stabilized at an axial position approximately $25 \mathrm{~mm}$ downstream of the burner face. water vapor are therefore essential for understanding atmospheric moistening and radiative processes.

An example of the observed interactions between large-scale turbulence structure and the flame is seen from the combined $\mathrm{CH} / \mathrm{CH}_{4}$ images in Fig. 1. The image to the left was taken during the first laser shot, $2 \mathrm{msec}$ before the image to the right. The high $\mathrm{CH}$ region, which defines the flame zone, is located in a narrow envelope located between $5 \mathrm{~mm}$ and 
$10 \mathrm{~mm}$ from the centerline in the mixing region along the periphery of the central jet where the fuel and air are mixed to near stoichiometric conditions[3]. The central fuel jet can be seen as the broader, lighter colored region located between the two flame zones. The exact radial location of the flame varies with both axial location and from shot to shot as the flame zone responds to variations in the fuel jet. Note that the high $\mathrm{CH}$ regions begin several millimeters downstream of the image bottom. These points correspond to the upstream location where the flame is stabilized, or the flame lift-off height. The flame lift-off height varies from shot to shot and is typically not the same on both sides of the jet.

Along the left edge of the jet in Fig. 1a the flame zone is relatively straight, with a small outward bulge near the center of the image at $x=40 \mathrm{~mm}$ where a vortical structure has begun to form. At this location the flame curves outward around the structure. Figure $1 \mathrm{~b}$, taken 2 msec later, shows further development of the structure and some downstream movement. In this case, no $\mathrm{CH}$ exists upstream of the structure. This behavior is consistent with the Broadwell model[4] in which extinction occurs along the upstream edge of vortical structures due to rapid entrainment of air. In this case, insufficient residence time is available for mixing between the entrained air and unburned fuel from the central jet and extinction of the flame occurs.

Another common interaction between the flame and a vortical structure is seen along the right side of the jet. Again the development of a vortical structure near the center of the image is apparent during the 2-msec delay between the two images. In this case the flame is not extinguished at the structure leading edge. Taken again in the context of the Broadwell model, it can be speculated that the reason for the difference between the two interactions is that, in the later case, mixing rates at the upstream edge of the structure are lower and the residence time sufficient to maintain combustion.

The above results clearly illustrate the interactions that occur between large-scale vortical structures along the periphery of the jet and the flame. Further analysis of the images shows that flame stabilization is largely determined by a balance between flame quenching due to turbulence interactions and upstream premixed flame propagation. These observations clearly establish the importance of unsteady, largescale turbulence interactions to flame stability and suggest that computational models for turbulent combustion must adequately account for such interactions when ignition and extinction dynamics are important.

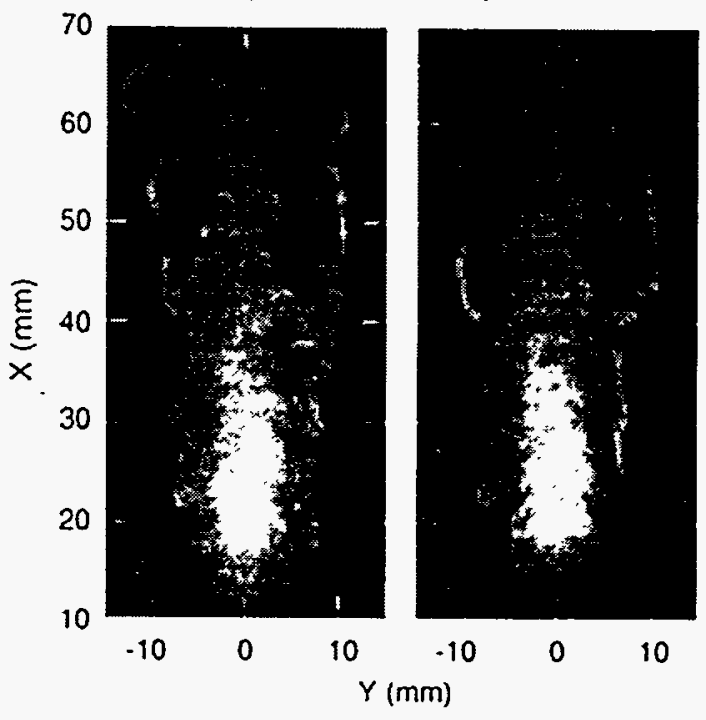

Figure 1. Double-pulse images of the instantaneous $\mathrm{CH} / \mathrm{CH}_{4}$ distribution in a turbulent, lifted $\mathrm{CH}_{4}$-jet flame. The grey scale is linear with highest concentrations indicated by lighter shades. The flow is from bottom to top with the fuel jet exit located 10 $\mathrm{mm}$ below the bottom of the images.

This work was supported by the Division of Chemical Sciences, Office of Basic Energy Sciences, U.S. Department of Energy, and by the Physical Sciences Department of the Gas Research Institute through Altex Technologies Corporation.

\section{References}

[1] W.M. Pitts, Twenty-Second Symposium Int'l) on Combustion (The Combustion Inst., Pittsburgh, PA, 1988), p. 809

[2] M. Namazian, R.W. Schefer, and J.Kelly, Twenty-Second Symposium Int'l) on Combustion (The Combustion Inst., Pittsburgh, PA, 1988), p. 627.

[3] R.W. Schefer, M. Namazian, and J. Kelly, "Stabilization of Lifted, Turbulent-Jet Flames", Comb.Flame, to appear, 1993.

[4] J.E. Broadwell, W.J.A. Dahm, and M.G. Mungal, Twentieth Symposium Int'l) on Combustion (The Combustion Inst., Pittsburgh, PA, 1985), p. 303. 


\title{
Finite-Rate and Transient Effects Studied in Direct Numerical Simulations of Turbulent Flames
}

\author{
Three-dimensional direct numerical simulations of turbulent non-premixed flames including \\ finite-rate chemistry and heat release effects have been performed. The results indicate that \\ unsteady strain rates experienced by the turbulent flame may be responsible for the observed \\ high concentrations of reaction intermediates.
}

\section{J. H. Chen, S. Mahalingam, ${ }^{1}$ and L. Vervisch ${ }^{2}$}

Turbulent flames are intrinsically difficult to model due to the strong coupling that exists between the chemical heat release and the fine-scale turbulent motion. Non-premixed flames are characterized by their internal structure and by the transport of fuel and oxidizer to the reaction zone where products of combustion are formed. If the chemical time scales are fast relative to the fluid mechanical time scales then the turbulent flame can be viewed as a random distribution of local chemical equilibrium problems. However, important physical effects such as extinction and pollutant formation can be successfully modeled only if nonequilibrium chemical effects are taken into consideration.

A significant role of direct numerical simulations (DNS) of turbulent combustion is to study in detail turbulence-chemistry interactions in completely defined situations. In DNS, all of the relevant flow and reactive scales are resolved and, therefore, no closure model is needed for the small scale mixing and chemical reaction. Both scalar and vector fields of interest to combustion modelers are attainable from post-processing the numerical databases. From these databases the effects of unsteadiness, differential diffusion, heat release, finite-rate chemical kinetics and curvature on the flame topology can be examined. In the present study of we have used DNS to study nonequilibrium and unsteadiness effects in turbulent nonpremixed flames. The present DNS account for finite-rate chemical kinetics and heat release effects. This study began as part of a summer research program held at the Center

1U. Colorado, Boulder, $\mathrm{CO}$.

2CORIA, INSA-Rouen, France. for Turbulence Research at Stanford University and NASA Ames Research Center in 1992.

To quantify finite-rate effects and to analyze the dynamics of extinction, the interaction between a non-premixed plane laminar flame and free-decaying turbulence was studied using DNS. The chemical kinetics were modeled with simplified one-and twostep Arrhenius kinetics. The two-step model was chosen to study the effect of a fastdiffusing radical-like species. The global Damkohler number was of order unity and the Taylor Reynolds number was fifty. The effect of the turbulence on the flame after twoeddy turnover times is shown in Fig. 1 . The initially plane laminar flame becomes stretched and distorted by the turbulence, and furthermore, pockets of extinction form in regions where the flame is unable to sustain the locally high tangential strain rates. Conversely, the effect of the flame on the turbulence is to create a zone of large viscous dissipation around the flame which has the effect of damping the turbulence.

DNS data suggest that the reaction zone structure of turbulent diffusion flames differs significantly from steady strained laminar flames. To facilitate comparison between laminar strained flame calculations using the one- and two-step chemical kinetics mechanisms and turbulent DNS results, the reaction rate at the stoichiometric surface was computed from the DNS database and plotted with respect to the inverse scalar dissipation rate, $\chi^{-1}$, shown in Fig. 2. As expected, the reaction rate increases with increasing scalar dissipation rate until a critical value is reached at which extinction occurs. Also shown in the 
figure is the response curve for a strained laminar flame. Significant deviations from the steady laminar flame boundary exist and are a result of the unsteadiness of the strain rate caused by the turbulence and the turbulence enhanced mixing which convects more reactants to the reaction zone than in a strained laminar flame. Furthermore, a substantial number of flamelets for dissipation rates larger than the laminar critical value exist, supporting the notion that a flamelet in a turbulent field can sustain excursions of the scalar dissipation rate above the laminar critical value.

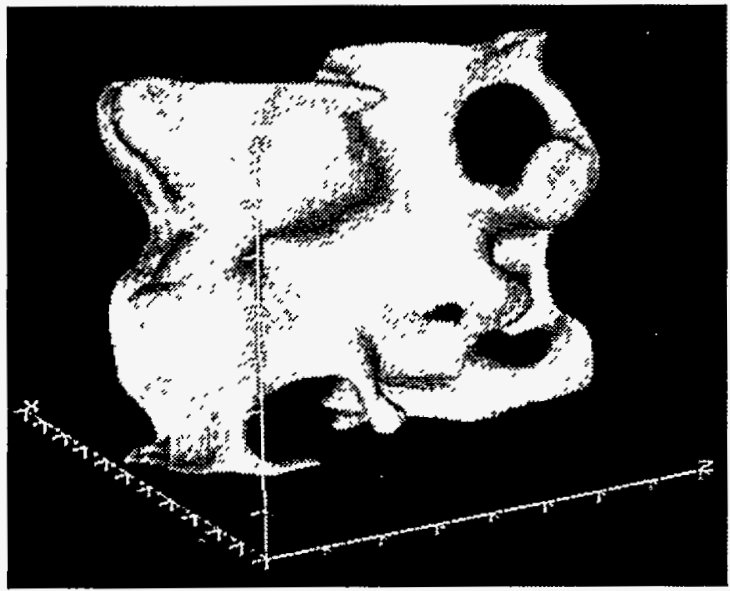

Figure 1. An isosurface of the instantaneous reaction rate for the two-step kinetics model. Holes correspond to local extinction

From a simplified two-step finite-rate chemical kinetics model[1], the concentration of an intermediate radical-like species $Y_{I}$ is determined in mixture fraction coordinates $Z$ for the turbulent DNS simulation and compared against steady strained laminar flame predictions. The comparison is shown in Fig. 3 and in that figure the peak concentration for the turbulent case is approximately twice that predicted by the strained laminar flame model. The abundance of intermediate species for the turbulent case is due to turbulence enhanced mixing which brings a wider range of concentrations of reactants to the reaction zone. Therefore, the results of the DNS suggest that the interaction of the finite-rate chemistry and turbulence can lead to a strong modification of the local response of the species concentrations and reaction rates compared with a strained laminar flame.

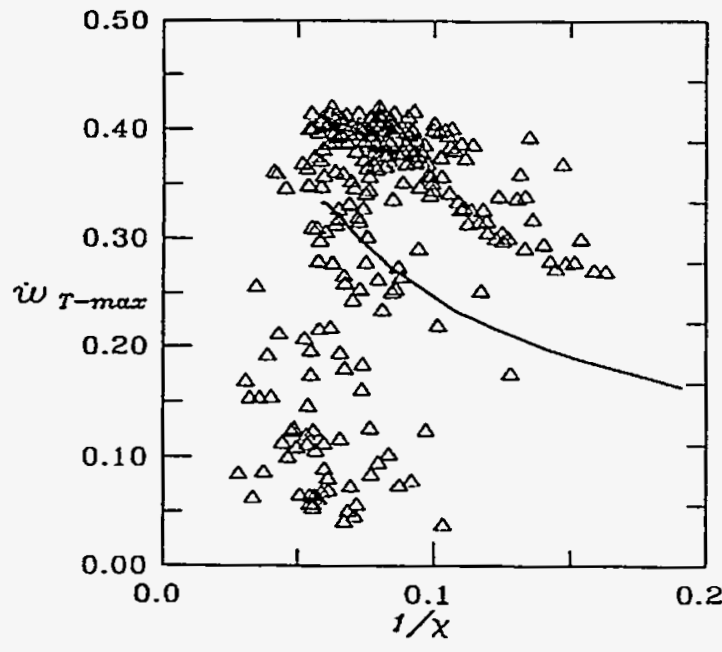

Figure 2. Distribution of maximum reaction rate (along the flame normal) with respect to inverse scalar dissipation rate. Triangles denote laminar strained flame predictions, crosses denote turbulence data.

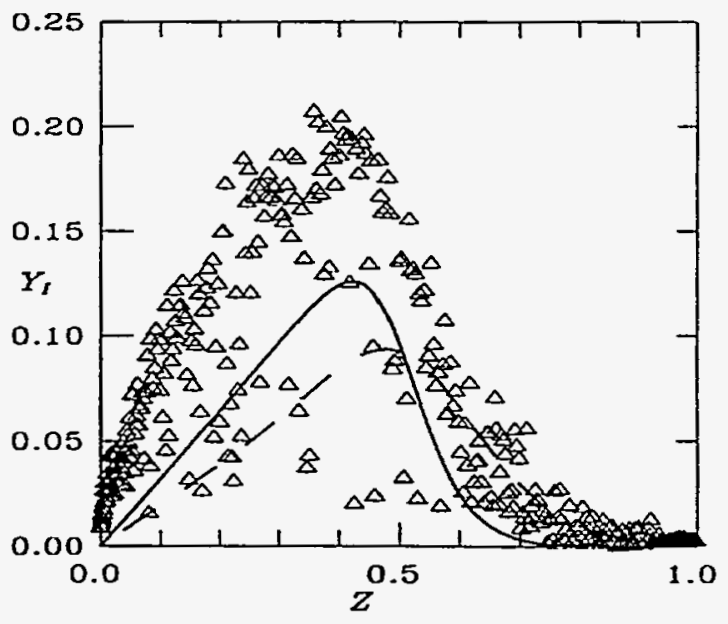

Figure 3. Intermediate species mass fraction versus mixture fraction. Steady strained laminar flame predic-tions for low (solid line) and high (broken line) strain rates. Triangles denote DNS of turbulent flame.

This work was supported by the Division of Chemical Sciences, Office of Basic Energy Sciences, U.S. Department of Energy.

\section{References}

[1] S. Mahalingham, J.H. Chen, and L. Vervisch, 1994. "Finite-Rate Chemistry and Transient Effects in Direct Numerical Simulations of Turbulent Non-premixed Flames," Combustion and Flame, (in press). 


\title{
Low Mach Number Reacting Flow Modeling
}

\author{
A numerical scheme for modeling low Mach-number reacting flow is developed using a \\ predictor-corrector projection method. Unsteady two-dimensional flame-flow interactions are \\ studied, revealing local finite rate effects on flame structure and reaction rate..
}

\section{H. N. Najm}

The detailed interaction of chemical reaction and fluid flow is key to the operating efficiency and emissions of most combustion systems. In particular, rates of generation of pollutants, e.g. nitrogen oxides and carbon monoxide, from premixed combustion systems such as automotive IC engines and utility gas turbines, are largely determined by the unsteady interaction between turbulent flow and flame dynamics.

It has been recently established that the essential spatial features of homogeneous turbulent flow involve quasi-two-dimensional (2D) tube-like structures of intense vorticity in three-dimensional (3D) space [1]. Therefore, it is evident that the understanding of the flameflow interaction involving unsteady 2D vortical structures and laminar flames can shed significant light on the more complicated turbulent flow-flame interaction in 3D. Furthermore, given that flame propagation velocities and flow speeds in many combustion applications are considerably less that the speed of sound, it is clear that acoustic dynamics can be eliminated from the reacting flow model used to study these systems, without impacting the physical processes of interest.

These are the key elements of the present approach: to develop low Mach-number reacting flow models, and utilize them for the study of unsteady $2 \mathrm{D}$ flame-flow interactions. This involves the formulation of the governing low Mach-number unsteady reacting flow equations using detailed chemical kinetics and viscous flow with variable transport properties. These equations are solved using an explicit finite difference predictor-corrector projection scheme. This scheme uses two fastPoisson solutions for the pressure field in each time step, and, given the predictor-corrector combination, is stable for typical temperature ratios found in reacting flow systems. Spatial and temporal discretization is second order using central and Adams-Bashforth differencing for the spatial and temporal derivatives respectively.

The second order convergence of the scheme has been demonstrated for the temporal evolution of a non-isothermal jet profile. The convergence data is presented in Fig. 1. Similar second order convergence is observed for a non-isothermal jet at high Reynolds number, in comparison with the jet growth from inviscid linear stability theory.

Single-step premixed reacting flow results with Arrhenius rate chemistry, are shown in the time sequence in Fig. 2. These plots illustrate the time development of the flame and flow structures in a doubly periodic domain, with pressure rise due to combustion heat release. The flow is initialized with a counter-rotating vortex pair situated in the cold reactants between two advancing premixed flame fronts. The contortion and elongation of the flame surface due to the vorticity field, as well as the generation of vorticity at the flame front due to baroclinic and dilatational effects are observed. As a result of the enhanced Flame front area, the integrated reaction rate in the domain is increased, leading to enhanced rate of pressure rise and heat release. Flame statistics reveal significant effects due to flow strain rate, stagnation pressure rise rate, and flame curvature on local flame thickness and burning rate. In agreement with other analytical and numerical results, strain is observed to decrease the local consumption rate of reactants at the flame front by reducing the flame thickness. Because of the pressure rise due to combustion, time history effects are 


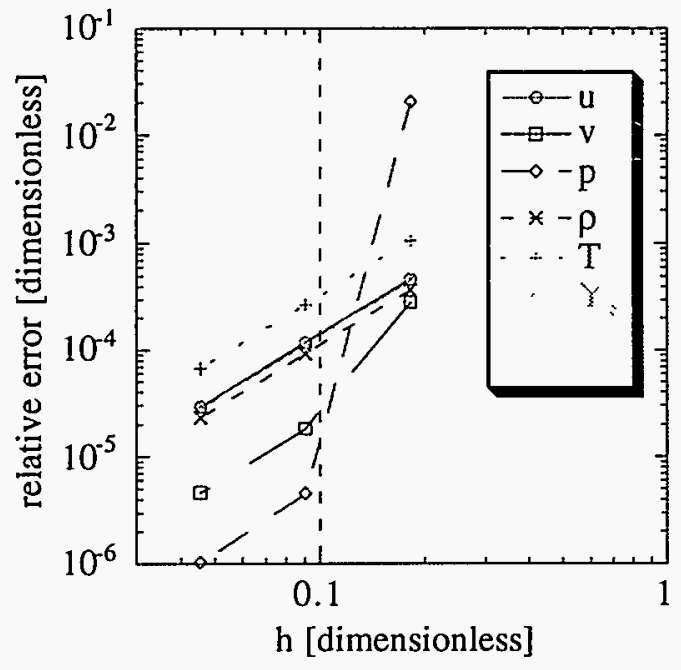

Figure 1. Second order convergence of the velocity $(U, V)$, pressure $(P)$, density $(\rho)$, temperature $(T)$, reactants mass fraction $\left(Y_{R}\right)$, and vorticity fields $(\omega)$, with spatial cell size, h.

significant, leading to a superadiabatic condition in the products.

Work is in progress for studying flames with full detailed chemical kinetics and variable transport properties in this and other geometries. comparison of detailed versus reduced kinetics will be used to study the validity of specific reduced chemical mechanisms in unsteady 2D vortical flow.

This work was supported by the Division of Chemical Sciences, Office of Basic Energy Sciences, U. S. Department of Energy.

\section{References}

[1] M.M. Rogers and P. Moin, (1987), "The Structure of the Vorticity Field in Homogeneous Turbulent Flows." J. Fluid Mech. 176, 33.
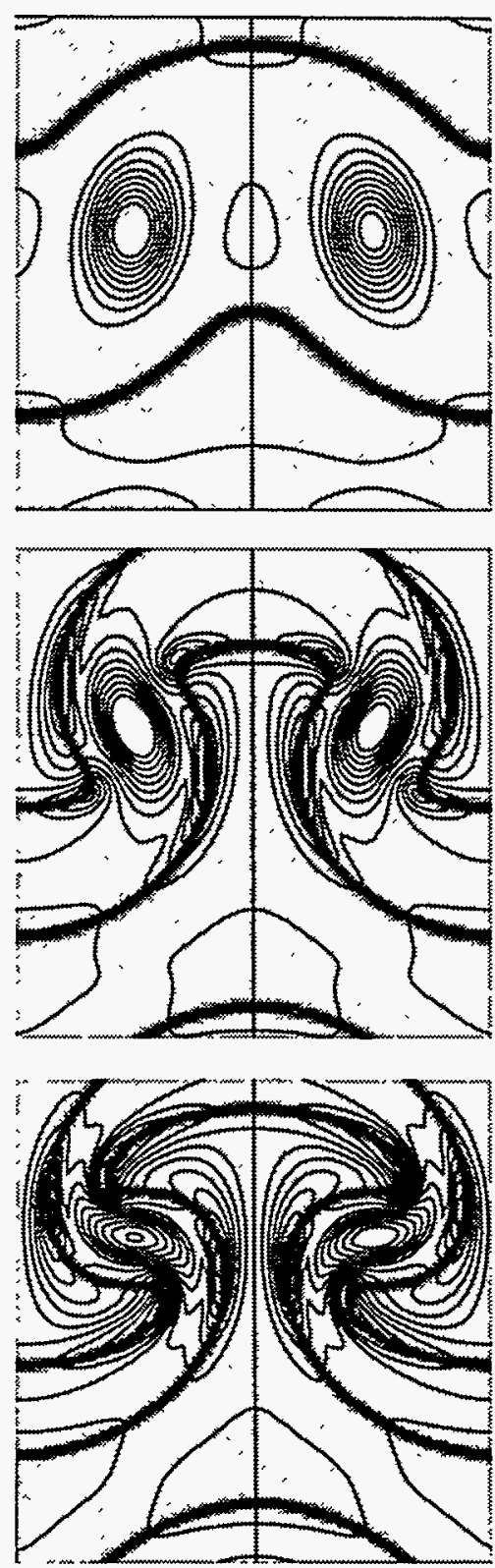

Figure 2. A time sequence showing the evolution of a counter rotating vortex pair and two premixed flame fronts. Contours delineate the vorticity field, while the reaction zone is shown shaded. The reaction fronts are burning inwards, consuming the cold reactants in between them. 


\title{
Premixed Flame-Speed Scaling in Low-Intensity Turbulence
}

\author{
A newly identified physical mechanism governing flame propagation through low-intensity \\ turbulence implies a modification of a widely accepted flame-speed scaling law, with \\ implications for high-intensity combustion and for sound-wave propagation through \\ turbulence.
}

\section{A. R. Kerstein and W. T. Ashurst}

The mechanism determining the premixed flame speed in a turbulent medium is generally considered to be best understood in the limit of small $v^{\prime} / \lambda$, where $v^{\prime}$ is the rootmean-square velocity fluctuation (a measure of turbulence intensity) and $\lambda$ is the burning velocity of the flame surface into the reactant. Neglecting heat release, local variations of $\lambda$, and related complications, the amplitude of a flame-surface undulation caused by a turbulent eddy is $v^{\prime} / \lambda$ times the wavelength of the undulation. The resulting increase of surface area can be estimated from trigonometry. The area increases by a factor of order $1+V^{\prime 2}$, where $V^{\prime}=v^{\prime} / \lambda$. It is well known that $\mathrm{u}_{\mathrm{T}} / \lambda$ is proportional to the area increase, where $u_{T}$ is the turbulent flame speed. The foregoing estimate therefore implies a flamespeed enhancement factor $\left(u_{T^{-}}-\lambda\right) / \lambda$ of order $\mathrm{V}^{2}$.

This widely accepted result omits consideration of the balance between processes tending to wrinkle the flame and those tending to smooth the flame. Smoothing is caused by the reduction of surface curvature during laminar burning. Our analysis of this competition indicates that wrinkling and smoothing processes are not in balance after the passage of an initially planar flame through a single turbulent eddy. Rather, the wrinkling process dominates until a balance is achieved at a larger value of $\left(u_{\mathrm{T}}-\lambda\right) / \lambda$. We find that the number of eddy passages required to achieve overall balance diverges as $\mathrm{V}^{\prime}$ approaches zero. The flame-speed enhancement factor at balance is predicted to be of order $\mathrm{V}^{4 / 3}$. The new scaling is confirmed by numerical simulations involving eddies arranged on periodic $2 \mathrm{D}$ and $3 \mathrm{D}$ lattices, but with randomly chosen sense of rotation to break the periodicity.

Nevertheless, the quadratic scaling is valid for the particular case of a spatially periodic flow field. In this case, undulations are selfcanceling over the course of one eddy passage, so there is no cumulative wrinkling effect. Prior tests of the scaling all involved numerical simulation of flame propagation through periodic flows, so the limited validity of the quadratic scaling was not noticed.

A model incorporating both geometrical aspects of flame surface evolution and statistical aspects of turbulent fluid motion has been formulated in order to predict the dependence of $u_{T}$ on aspects of flow structure other than $V^{\prime}$. The model consists of a pair of randomly forced differential equations representing the motion of two points on the flame surface separated by one eddy diameter. The model reproduces the $\mathrm{V}^{4 / 3}$ scaling and predicts additional dependencies.

The additional predictions are illustrated by another class of flows that have been simulated numerically. This class consists of flows that are periodic (in particular, sinusoidal) at any given instant, with velocity aligned parallel to a selected Cartesian axis. The velocity fluctuation amplitude varies sinusoidally with time. Whenever the amplitude becomes zero, the velocity orientation axis and the axis corresponding to the direction of flow variation are randomly reassigned, and a random spatial displacement is applied.

This class of flows is parametrized by a normalized "phase velocity" $\mathrm{P}=\mathrm{W} /\left(\mathrm{Tv}^{\prime}\right)$, where $W$ is the period of the spatial variation, 
$\mathrm{T}$ is the period of the time variation, and $\mathrm{v}^{\prime}$ is the time-average velocity fluctuation amplitude. For this class of flows, the model predicts $\left(u_{\mathrm{T}^{-}}-\lambda\right) / \lambda=\mathrm{cP}^{-2 / 3} \mathrm{~V}^{4 / 3}$, where $\mathrm{c}$ is an unknown proportionality constant.

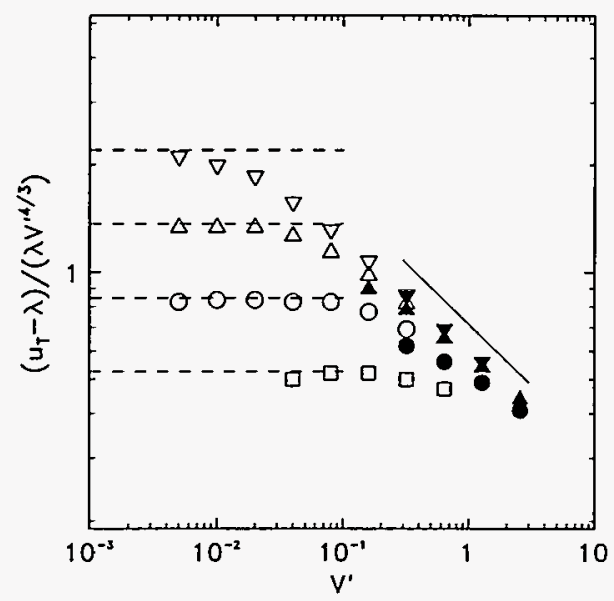

Figure 1. Scaled flame-speed enhancement factor versus scaled turbulence intensity $V^{\prime}$ for simulated flows parametrized by the normalized phase velocity $P$. Profiles, distinguished by plot symbols, correspond (top to bottom) to $\mathrm{P}=1 / 4,1 / 2,1$, and 2. Dashed lines: model results in the low-V' limit, based on a one-parameter fit. Solid line corresponds to a linear dependence of flame-speed enhancement on 'V'.

Fig. 1 shows results of 2D numerical simulations involving this class of flows for $\mathrm{P}$ values ranging over a factor of 8 . The horizontal dashed lines are predictions of the small-V' behavior with $c$ chosen to give the overall best fit. On the scaled plot, the approach to a plateau at low $\mathrm{V}^{\prime}$ is indicative of the $\mathrm{V}^{4 / 3}$ scaling. The correspondence of simulation and model plateau levels is indicative of the $\mathrm{P}^{-2 / 3}$ scaling. 3D simulations were also performed and likewise confirm the scalings.

The simulations extend to $\mathrm{V}^{\prime}$ values large enough to capture the linear dependence of $\mathrm{u}_{\mathrm{T}}$ on $\mathrm{V}^{\prime}$ that is known to govern the highturbulence-intensity limit. No single computational method is reliable and efficient over the whole $V^{\prime}$ range, so two methods were used. One (open symbols) is valid at small $\mathrm{V}^{\prime}$ and the other (filled symbols) is valid at large $V^{\prime}$. The numerical agreement of the two methods at intermediate $V^{\prime}$ suggests that a reasonably accurate representation of the complete $V^{\prime}$ dependence has been achieved. This computation is the first to demonstrate the complete $\mathrm{V}^{\prime}$ dependence for a given flow structure. As such, it provides a benchmark for future model development.

The low-turbulence-intensity analysis has implications for modeling the more important, and more intractable, high-intensity regime. The renormalization-group analysis subdivides high-intensity flame propagation into a hierarchy of low-intensity subprocesses, each governed by the low-intensity scaling. The new result suggests a modification of a previous renormalization-group analysis that was based on the quadratic low-intensity scaling. However, it is likely that neither the previous nor the modified formulation is valid because the method does not appear to reflect the large length scale over which the wrinkling-smoothing balance is established.

The low-intensity scaling is applicable to the passage rate of acoustic impulses through the ocean, owing to the slowness of currents compared to the sound speed. Possible relevance to ongoing ocean acoustic tomography studies is under investigation.

This work supported Division of Engineering and Geosciences, and Division of Chemical Sciences, Office of Basic Energy Sciences, U. S. Department of Energy. 


\title{
Reacting Flows
}

\section{Growth of a Turbulent Flame}

\author{
Flame propagation within an engine occurs during a fixed interval of engine rotation, \\ over all operating speeds. A simple numerical model is presented that duplicates this \\ essential engine feature.
}

\section{W. T. Ashurst}

After the spark, what happens in a car engine? The fuel and air have been mixed to the desired ratio but how does the flame move across the chamber in the time period before the piston motion creates an expanding volume? Without the pressure rise due to combustion there would be no push on the piston and no power from the engine. The fact that flame motion increases with engine speed has been known since the early development of internal combustion engines, but just how this occurs and how to modify the process to affect pollution formation is not well known. Turbulence in the unburnt gas mixture causes the increased flame speed, as suggested by Damkohler fifty years ago, but further progress in our detailed understanding has been slow. It appears that the answer may be that turbulence distorts the shape of the flame and this distortion, combined with volume expansion, results in an exponential growth rate.

Consider first a premixed flame burning into a mixture with no turbulence and no chamber walls. In this idealized case, a spherical flame would move our from a point ignition with a constant velocity -- the velocity given by how much volume expansion occurs for each volume of gas that is consumed by the flame. Outside, and away from this flame, there is a flow created within the unburnt gas, this flow moves the unburnt gas away from the flame in order to accommodate the newly created volume of burnt gas. The flow speeds are so small in comparison to the sound speed that motion away from the flame is almost incompressible flow, and thus, conservation of volume flux in the unburnt gas determines the spatial nature of this gas motion. For a spherical flame, the velocity at distance $R$ is $V(R)=\tau S_{L} R_{S}^{2} / R^{2}$ where $R_{S}$ the spherical flame radius and $\tau$ is the volume created per unit volume consumed by a flame moving at speed $S_{L}$ into the mixture in front of the flame. It is important to notice that at the flame location, the velocity created by volume expansion does not depend upon the size of the flame, that is, for $R=R_{S}, V(R)=\tau S_{L}$. Thus, the idealized spherical flame moves at a constant speed, and if this occurred in an engine, then less and less of the mixture would be reacted as the engine speed increased.

We do know from flame pictures within an engine that the flame is not a smooth spherical surface, but appears more like the edge of a cloud; these distortions are caused by the turbulent motion of the unburnt gas. Computed flame motion in two dimensions are shown in Fig. 1. Now, if the turbulent flame surface is very distorted, what is the effect upon the velocity field created by volume expansion?

We assume that the flame surface is very distorted, and so, between some small radius $R_{1}$ and the largest flame radius $R_{2}$, there will be a distribution of flame surface. Thus, over a spherical surface $R_{S}$, which lies between $R_{1}$ and $R_{2}$, there will be some average value of volume creation. This distributed flame can be characterized by specifying the flame area per unit volume, or the flame surface density. Our first guess for a turbulent flame ball is to use the functional form of the velocity field created by a thin spherical flame with radius $R_{S}$ but reduce the magnitude to a level associated with the amount of flame area that occurs within the volume between $R_{S}$ and $R_{S}+d R_{S}$. The total flow velocity induced by this 
distorted flame surface is given by summing over spherical shells as

$$
V(R)=\tau S_{L} \int_{R_{1}}^{R_{2}} \Sigma\left(R_{s}\right)\left(R_{S}^{2} / R^{2}\right) d R_{S}
$$

Where $\Sigma\left(R_{S}\right)$ is the flame surface density and has dimensions of inverse length. From other work, we set $\Sigma\left(R_{S}\right)=(1 / \lambda) \sqrt{u^{\prime} / S_{L}}$ where $\lambda$ is the Taylor length scale of the unburnt gas turbulence and $u^{\prime}$ is its root mean square velocity. The Taylor length scale can be defined by the mechanical energy dissipation caused by the turbulent motion as $\varepsilon=15 v u^{\prime 2} / \lambda^{2}$ where $v$ is the kinematisity and $\varepsilon$ is the kinetic energy decay per unit mass. With this assumption for $\Sigma\left(R_{S}\right)$, the velocity created by volume expansion at the largest flame radius $R_{2}$ is

$$
V\left(R_{2}\right)=\left(\tau S_{L} R_{2} / 3 \lambda\right) \sqrt{\left(u^{\prime} / S_{L}\right)}\left(1-\left(R_{1} / R_{2}\right)^{3}\right)
$$

Now if $R_{1}$ is small compared to $R_{2}$, then the last term is a constant of order unity and we see that distributed volume expansion creates a velocity which is proportional to the largest flame radius. We can also express the turbulence length scale in terms of the integral scale $L$, and using the approximation that $\varepsilon \approx u^{\prime 3} / L$, yields $\lambda / L$ as $15 v u^{\prime} / \lambda$. Also, the flame burning velocity and thermal diffusivity determine the flame thickness $\delta_{f}$ and using the kinematic viscosity in place of the thermal diffusivity, gives $v \approx \delta_{f} S_{L}$. These two approximations when used in the above relation, and ignoring numerical constants, gives a flame velocity of $V(R)=\tau S_{L}-R u^{\prime} / \sqrt{L \delta_{f}}$ where the linearity in $u^{\prime}$ occurs from the product of two square-root terms. Integration of this result leads to exponential growth behavior, and the growth rate will be proportional to $u^{\prime}$. The flame size in terms of $r_{o}=\sqrt{L \delta_{f}} / \tau$ will be $R(t) / R(0) \approx \exp \left(u^{\prime} t / r_{d}\right)$ where $R(0)$ is the flame size at the start of the exponential growth behavior.

A feature of engine flow is that $u^{\prime}$ is proportional to engine speed, and so while the time available for combustion changes with speed, the combination $u^{\prime} t / r_{0}$ is approximately constant over a range of engine speeds. Thus, the flame motion will always be fast enough so that the flame fills the chamber within a given period of engine rotation. While increasing the engine speed decreases the available time period, this is compensated by the increased $u^{\prime}$. This flame behavior is a result of distributed volume expansion which creates exponential growth of flame area.

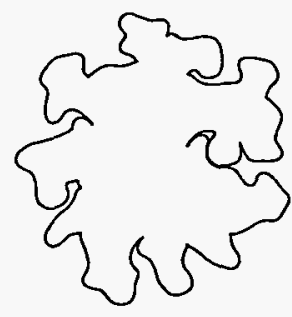

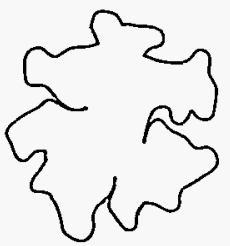

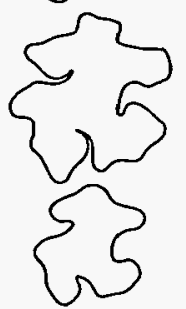<smiles>C1CCCC1</smiles>

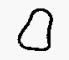

Figure 1. Computed turbulent flame growth in two dimensions. The six images are equally spaced in time and show the distortion caused by the unburnt gas turbulence. The last (upper) two images are distorted enough so that the exponential growth rate has started.

This work supported by the Division of Chemical Sciences, Office of Basic Energy Sciences, U. S. Department of Energy.

\section{References}

[1] W.T. Ashurst, "A Simple Illustration of Turbulent Flame Ball Growth", to appear in Combustion, Science \& Technology, (1995). 


\title{
The Deflagration of Porous Energetic Materials
}

\author{
A theoretical model has been developed to describe the multiphase deflagration of porous
} energetic solids, such as degraded nitramine propellants. The presence of gas flow relative to the condensed material in the two-phase regions is shown to affect virtually all aspects of the deflagration wave.

\section{S. B. Margolis and F. A. Williams ${ }^{1}$}

The combustion behavior of energetic materials has long been of interest in the fields of propulsion and pyrotechnics. In many such applications, it is becoming increasingly clear that two-phase-flow effects play an important role. During combustion of most homogeneous solid propellants, for example, thin multiphase layers develop at their exposed surfaces, where finite-rate exothermic reactions occur. There is also growing interest in the combustion behavior of porous energetic solids, since even initiaily dense materials can develop significant void fractions if, at any time, they are exposed to abnormal thermal environments. The deflagration characteristics of such "damaged" materials may then differ significantly from those of the pristine material due, at least in part, to gas flow in the solid/gas preheat region. The presence of gas in the porous solid results in a more pronounced two-phase effect in the multiphase surface layer. Burning nitramine propellants, for example, tend to exhibit extensive bubbling in an exothermic foam layer that forms in the liquid melt region.

A time-dependent, multidimensional mathematical model has been derived that is capable of describing ignition and deflagration (Fig. 1) in porous energetic materials [1,2]. Some of the features of the model, which, is applicable to nitramines such as HMX, include melting of the initially solid material, twophase-flow effects in both the solid/gas and liquid/gas regions, and differences between the condensed- and gas-phase velocities and temperatures. Following ignition, or cookoff, it is presumed that a deflagration wave develops that is characterized by a melting front followed by combustion in a relatively thin two-

\footnotetext{
${ }^{1}$ University of California, San Diego, California
}

phase region consisting of the unburned liquid material and gas-phase products of combustion.

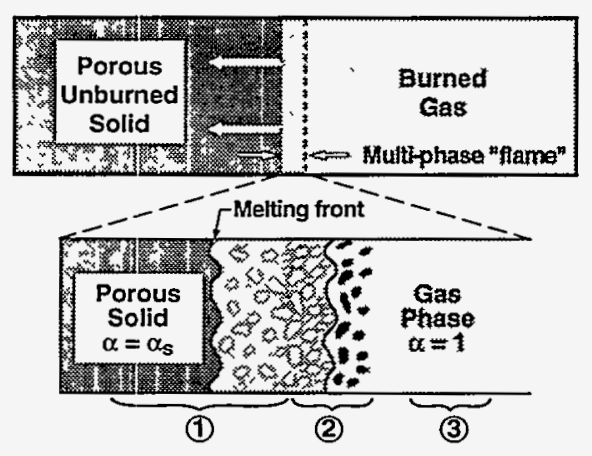

Figure 1. Deflagration of a porous energetic material with two-phase flow in both the solid/gas and liquid/gas regions, with combustion occurring in the latter. The lower figure shows the details of the multi-phase "flame" structure, consisting minimally of (1) a preheat zone containing a melting front across which the porous solid changes into a bubbly liquid, or foam, and (2) a thin liquid/gas reaction zone. Additional gas-phase reactions may occur in a secondary gas-flame region (3) downstream from the primary twophase reaction zone (2).

An analysis of this model, based on the application of activation-energy asymptotics to derive a dynamic flame-sheet type of approximation for the relatively thin reaction zone, has led to a number of analytical predictions. These results serve to characterize the nature of an unconfined, freely propagating deflagration, and include expressions for the final burned temperature and the steady deflagration speed as a function of pressure, for various values of the initial porosity and other material properties. Predictions of intrinsic instability due to two-phase thermal/diffusive 


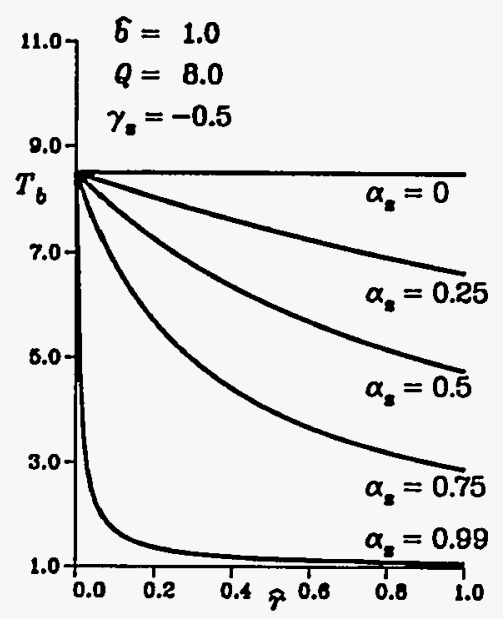

Figure 2. Final burned temperature $T_{b}$ as a function of the gas-to-solid density ratio $\hat{r}$ where the latter is proportional to the gasphase pressure, for several values of the initial gas-phase volume fraction $\alpha_{s}$.

effects have also been obtained. With respect to steady deflagration, these studies demonstrate how most aspects of the deflagration wave, including its structure, propagation speed, and final temperature, depend on the local pressure in the two-phase region ( $c f$. Figures 2,3 ). Another capability of our model is its ability to assess the influence of the thermal conductivity of each phase on the deflagration velocity. In particular, although this quantity increases as the thermal conductivity of either phase increases, the thermal conductivity of the phase having the higher value of this parameter exerts the greater influence on the burning rate. This result follows from the fact that a higher proportion of the heat is transferred through the more highly conducting phase.

Regarding the stability of steady, planar deflagration with respect to nonsteady, nonplanar perturbations, the present study has shown that there is a pulsating neutral stability boundary in the space of parameters, similar to that obtained in gasless combustion (combustion synthesis) and in nonporous solid-propellant combustion. Beyond this threshold, infinitesimal perturbations about the steady, planar state can be expected to grow to finite amplitude, thus giving rise to a nonsteady, nonplanar mode of burning. One

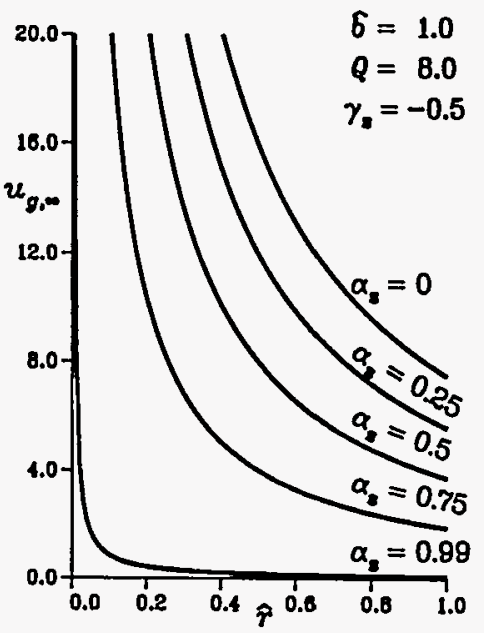

Figure 3. Final gas velocity $u_{g, \infty}$ as a function of $\hat{r}$ for several values of $a_{S}$.

particularly important conclusion is that the effect of an initial nonzero porosity $a_{S}$ is generally destabilizing, by an amount proportional to $a_{S}\left(1-a_{S}\right)^{-1}$, relative to the nonporous case. This effect arises both from the lower combustion temperature of the porous energetic material and from the gas-phase diffusion of heat from the reaction zone towards the porous preheat region. This result suggests that degraded energetic materials, which exhibit substantially greater porosity than their undamaged counterparts, may be especially prone to time-dependent, multidimensional (e.g., spinning) deflagration. These and other related results are described in further detail in the two references listed below.

This work was sponsored by the U.S. Department of Energy and by a Memorandum of Understanding between the Office of Munitions (Department of Defense) and the Department of Energy.

\section{References}

[1] Margolis, S. B., and Williams, F. A., SAND94-8649/AIAA Paper No. 95-0600, to appear in Journal of Propulsion and Power, 1995.

[2] Margolis, S. B., and Williams, F. A., SAND95-8409, submitted for publication, 1995. 



\section{Combustion in Engines and Commercial Burners}

\section{Introduction}

The Department of Energy (DOE) and other funding agencies sponsor a broad spectrum of applied research at the Combustion Research Facility (CRF) for the advancement of engines and commercial burners. This work is done in partnership with industrial collaborators.

At the CRF, we work on a variety of research projects to promote improvements in internal combustion engines. Our efforts are focused on increasing U.S. competitiveness through enhanced energy efficiency and reduced environmental impact. Sponsored by the DOE Office of Transportation Technologies (OTT), we are working with the Low Emissions Partnership under the United States Council for Automotive Research (USCAR) to meet the stringent $\mathrm{CO}$ and hydrocarbon emission levels imposed by the 1990 amendments to the Clean Air Act. For the Advanced Research Projects Agency (ARPA), our research has concentrated on the development of high pressure fuel injection in diesel engines to improve the energy efficiency and performance. Under the sponsorship of Defense Programs for dual use applications, studies of the fundamental mechanisms of soot and $\mathrm{NO}_{x}$ formation in diesel engines are dramatically changing conventional wisdom of diesel combustion typically embraced by the industry. In a program sponsored by Office of Utility Technologies (OUT) we are developing systems to illustrate and facilitate the application of hydrogen as an alternative energy carrier. In this multi-laboratory effort we are developing hydrogen based propulsion systems that meet or exceed the Ultra-Low Emission Vehicle requirements.

Diversified research at the CRF into commercial combustion processes is a strong contributor to U.S. industrial needs. Sandia recently opened the Burner Engineering Research Laboratory (BERL). This unique user facility is funded in collaboration with the Gas Research Institute (GRI) and the DOE Office of Industrial Technologies (OIT). Manufacturers of industrial burners bring their burners to the BERL and, in collaboration with researchers at the CRF, these burners are evaluated to obtain a basic scientific understanding of burner technology. A prime example of the BERL utility is our Petroleum Environmental Research Forum project. In collaboration with seven industrial partners and through individual Cooperative Research and Development Agreements, we are investigating the origin and fate of air toxics from oil refinery process heaters. In another OIT project, key contributions have been made to the advancement of burner technology through our internationally recognized fundamental research into sprays and spray combustion. With OIT sponsorship, Sandia has also been a world leader in understanding the fundamentals of pulse combustion processes. Our most recent results have shown ultra-low levels ( $5 \mathrm{ppm}$ ) of $\mathrm{NO}_{x}$ and $\mathrm{CO}$ emission are possible without aftertreatment. Under GRI sponsorship, we are developing and have released initial versions of the Sandia Pulse Combustion Design Code. This is a Microsoft Windows package designed to assist industry in the development of pulse combustion systems suitable for commercial applications. In another, OUT sponsored project, we are developing lean premixed combustion technologies to facilitate the use of hydrogen in a safe and environmentally benign fashion.

Our close connection with the industrial community has enabled the CRF to make substantial contributions to the U.S. advancement in commercial burners resulting in more efficient and cleaner processes.

Focusing on the interface between pure research and industrial application, programs in engines and commercial burners continues to advance the DOE objective of bringing industrial and national laboratory collaborative research to the marketplace.

\section{R. W. Carling, C. M. Hartwig, and J. O. Keller}




\title{
Quantitative Vapor-Fuel Imaging in a DI Diesel Engine using Planar Laser Rayleigh Scattering ${ }^{*}$
}

\author{
Quantitative images of vapor-phase fuel concentrations were obtained in an evaporating and \\ combusting Diesel jet using planar laser Rayleigh scattering. The images show that just \\ prior to first measurable heat release the fuel and the air are relatively well mixed throughout \\ the leading portion of the Diesel jet with the equivalence ratio ranging from 2 to 4 .
}

\section{Espey, ${ }^{1}$ J. E. Dec, and E. L. Porter}

Combustion performance and emissions of Diesel engines are strongly influenced by the fuel-air mixing. In order to understand, and subsequently optimize, the turbulent fuel-air mixing process, quantitative planar measurements of the fuel distribution under Diesel engine conditions are needed.

To obtain quantitative fuel-vapor concentration images, the authors recently introduced the application of planar laser Rayleigh scattering (PLRS) to a firing direct-injection (DI) Diesel engine [1]. Rayleigh scattering is an attractive diagnostic since it requires no doping of the fuel with either particles or tracer molecules.

A key issue in using Rayleigh scattering for quantitative fuel-vapor concentration imaging in the engine is that there must be no significant elastic (Mie) scattering from liquid fuel droplets and/or dirt particles. Mie scattering is generally many times stronger than the Rayleigh scattering from fuel-vapor molecules. Therefore, PLIR images can only be obtained in the pure vapor-phase regions of the Diesel fuel jet, and both the fuel and air must be free of dirt particles.

A previous study [1] showed, that the liquid-phase fuel of the Diesel jet evaporates very rapidly, and that beyond $27 \mathrm{~mm}$ from the injector all the fuel is entirely vapor phase. This was done by investigating the Diesel jet under high magnification using 2-D elasticscatter imaging and evaluating the signal intensities of droplets and solid particles of

\footnotetext{
* Adapted from an article submitted to Combustion and Flame, 1994.

1 Pennsylvania State Univ., University Park, PA.
}

progressively smaller sizes to determine the resolution of the imaging system.

The PLRS measurements were conduced in an optically accessible heavy-duty DI Diesel engine under fired and non-fired conditions. The intake air conditions were set so that motored TDC (top dead center) temperatures and pressures (1000K and $5.0 \mathrm{MPa})$ as well as ignition delay and premixed burn fraction matched those of the corresponding production engine.

The optical configuration is shown in Fig. 1. The horizontal laser sheet entered the combustion chamber through one side window and intercepted the vapor-phase region of the fuel jet. The PLRS images were obtained through the window in the cylinder head with an intensified video camera. The measurements of the fuel jet were conducted in a region beyond $27 \mathrm{~mm}$ from the injector nozzle, where all the fuel is vapor phase. This corresponds to the main combustion zone in this engine [2].

The images in Fig. 2 show the evolution of the fuel distribution for both the reacting (top) and the nonreacting (bottom) jet. Up to $4.5^{\circ}$ after start of injection (ASI), the general appearance of the fuel distributions of the reacting and nonreacting jets are very similar. For both cases, at $4.5^{\circ} \mathrm{ASI}$, the equivalence ratio within the jet ranges mostly from $2-4$. The edges of the jet at the sides and the front are well defined by a sharp decline from the equivalence ratio levels within the jet down to the background. Only the outline of the cross section of the turbulent jet differs from image to image due to cycle-to-cycle variations.

At $5.0^{\circ}$ ASI, immediately after the first indicated heat release, the two sequences di- 
verge. In the reacting jet, the Rayleigh signal intensity has decreased significantly from the levels at $4.5^{\circ}$. This signal reduction is mainly caused by the decrease in the Rayleigh scattering cross-section due to fuel breakdown at ignition and it occurs throughout the leading portion of the reacting jet.

After $5.0^{\circ}$ ASI in the non-reacting jet, the overall equivalence ratio in the front of the jet decreases due to continuous fuel-air mixing. At later crank angles, a greater variation of the fuel distribution within the jet can be observed. Particularly in the leading portion of the jet, the decline from the high equivalence ratios to low equivalence ratios is more gradual than observed in the first three images of this sequence. However, at the front edge of the jet there is still a sharp transition from equivalence ratio levels in the jet down to the level of the surrounding air.

The comparison of the Rayleigh images in Fig. 2 shows not only the effect of ignition and combustion on the Diesel fuel jet, but also the limits of this diagnostic for obtaining quantitative fuel concentrations in the reacting jet. Until significant fuel breakdown occurs, the Rayleigh scattering intensity is directly related to the fuel concentration, thus the images are quantitative. Fuel breakdown becomes significant in the reacting jet after $4.5^{\circ}$ ASI, and the images are no longer quantitative. For the non-reacting case, the entire image sequence is quantitative, and it provides an accurate 2-D measurement of a transient, vaporizing fuel jet under realistic Diesel conditions.

This work was supported by the Cummins Engine Company and the U.S. Dept. of Energy: Advanced Industrial Concepts Division, and Defense Programs, Technology Transfer Division.

\section{References}

[1] Espey, C., Dec, J.E., Litzinger, T.A. and Santavicca, D.A., SAE paper no. 940682, 1994.

[2] Espey, C. and Dec, J.E., SAE Transactions, 102, sec. 4, pp. 703-723, paper 930971, 1993.

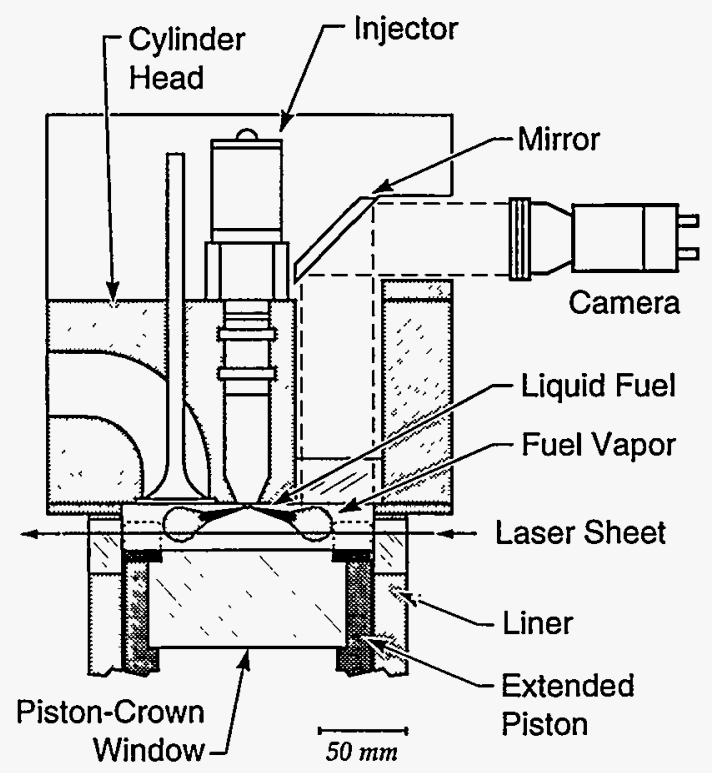

Figure 1. Engine schematic and optical setup. $4.0^{\circ} \mathrm{ASI}$

$4.5^{\circ} \mathrm{ASI}$ $5.5^{\circ} \mathrm{ASI}$

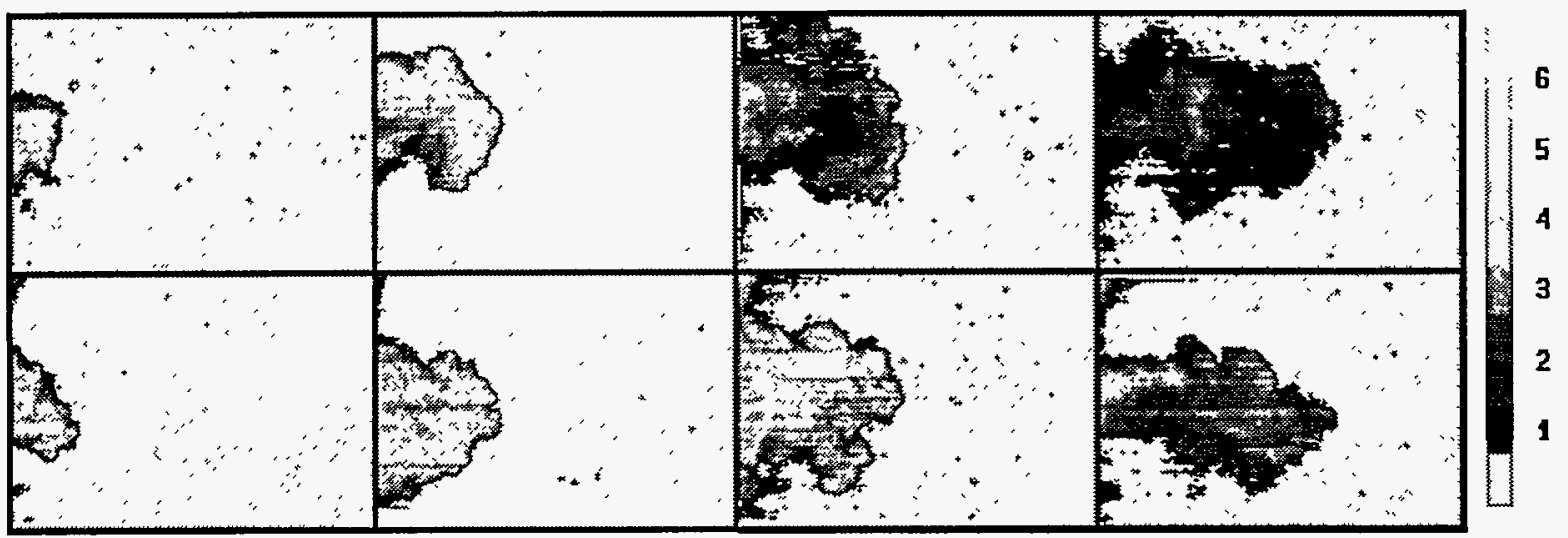

Figure 2. Temporal sequence of equivalence ratio images of the leading portion of the Diesel jet. The reacting jet is shown in the top row and the nonreacting jet in the bottom row. The equivalence ratios have been mapped to a gray scale as shown at the right. The crank angle degree ASI is given above each image pair. The left edge of each image is $27 \mathrm{~mm}$ from the injector, and the field of view is $18.1 \mathrm{~mm}$ by $12.5 \mathrm{~mm}$. 


\title{
Comparison of Crankcase and Blower Scavenging in a Fired Two-Stroke Cycle Engine
}

\author{
The in-cylinder flow field of a Schnürle (loop) scavenged two-stroke engine has been \\ examined for both blower and crankcase driven scavenging. Mean velocity profiles, RMS \\ velocity fluctuations and combustion performance show a strong dependence on the \\ scavenging method.
}

\section{P. C. Miles, R. M. Green and P. O. Witze}

Recently, the engine research and development community renewed its interest in twostroke engine technology. Many manufacturers are currently testing new engine designs on dynomometer stands and in prototype vehicles. This development activity has resulted in a large amount of attention being given to the scavenging process which is so critical to the operation of this type of engine.

Scavenging refers to the displacement of burnt gases in the cylinder by fresh fuel-air mixture. Two very different modes of scavenging are currently being considered in contemporary engine designs: crankcase scavenging, where the downward motion of the piston compresses the air in the crankcase and forces it into the cylinder, and external blower scavenging, where a separate pump delivers air to the cylinder.

Our objective in performing this work was to characterize and compare the in-cylinder flow fields associated with both crankcase and external blower scavenging configurations, in an engine that can be operated in either mode. We also assessed combustion performance for each scavenging technique.

In the experiments carried out in this study, the engine was fueled with stoichiometric, premixed propane and air and operated at $1200 \mathrm{RPM}$ for delivery ratios of $0.4,0.6$, and 0.8 . The delivery ratio is a measure of the mass of fuel-air mixture supplied to the engine; delivery ratios of $0.4-0.8$ correspond to light-to-medium load conditions. Our primary diagnostic was a fiber-optic based LDV system, which we used to measure the radial component of velocity along the cylinder centerline during both motored and fired operation. LDV seed particles $\left(\mathrm{TiO}_{2}\right)$ were generated from the thermal decomposition of $\mathrm{Ti}\left(\mathrm{OC}_{3} \mathrm{H}_{7}\right)_{4}$. The LDV system, making measurements inside the engine cylinder, yielded data rates in the $5-20 \mathrm{kHz}$ range.

Typical mean velocity results at the time when the intake ports are approximately onethird open are shown in Figure 1. Comparing the motored data with the fired data and noting the dramatic differences between the two, it should be clear that there is no benefit in studying the scavenging flow field in a motored engine.

\section{Fired, DR=0.60: $\overrightarrow{50(\mathrm{~m} / \mathrm{s})}$}

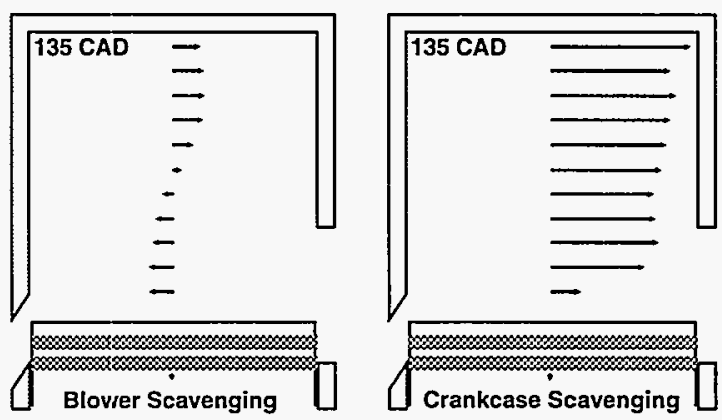

Motored, DR=0.60: $\overline{50(\mathrm{~m} / \mathrm{s})}$

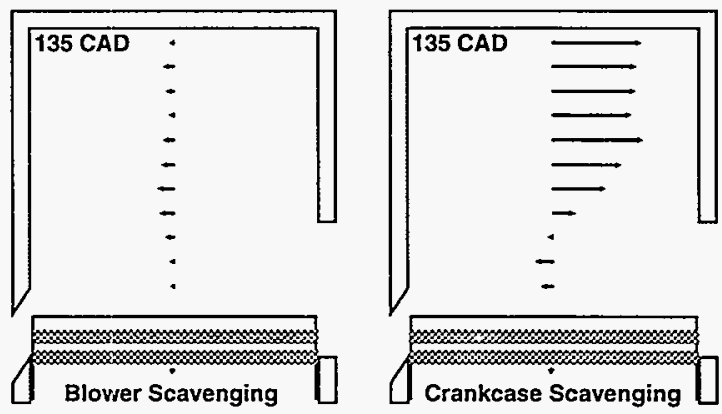

Figure 1 Profiles of the radial component of velocity along the cylinder centerline. 
For fired engine operation, the major features of the flow fields are quite insensitive to changes in delivery ratio. While the phasing and magnitude of these features do vary with delivery ratio, the general nature of the flows do not. On the other hand, considerable differences in the flow fields are observed between blower and crankcase scavenging, as is illustrated in Figure 1. These differences persist throughout the cycle.

During cylinder blowdown, the velocity fields are quite similar for both scavenging methods, and blowdown is complete well before intake port opening. We observed a backflow into the cylinder following blowdown which persists until the intake port opens. This is due primarily to continued cylinder volume expansion with piston motion, with secondary influence from pressure dynamics in the exhaust manifold.

Early in the scavenging process, both the blower scavenged and the crankcase scavenged flows are characterized by a dominant tumble-vortex structure. The crankcase scavenged flow subsequently evolves to a structure characterized by plug-like flow toward the exhaust port. For both cases, the fresh charge appears to penetrate to the top of the combustion chamber. Both may be suitable for efficient scavenging of 'bowl-in-head' combustion chamber designs. This penetration grows stronger with increasing delivery ratio.

Later in the scavenging process, the blower scavenged flow evolves to one characterized by a uniform positive radial velocity profile, followed by the formation of energetic, large-scale tumble motions due to the continued delivery of fresh charge. For crankcase scavenging, the flow reverts to a rotating, tumble vortex pattern significantly weaker than observed for blower scavenging.

Finally, during compression of the largescale structures set up late in the scavenging process by the blower scavenged flow, higher shear in the axial direction is produced. This high shear probably results in enhanced production of turbulence. The RMS velocity fluctuations near the top of the combustion chamber confirm this enhanced production of turbulence. The RMS velocities in the case of blower scavenging are consistently higher and indicate significant spatial variations in contrast to the case of crankcase scavenging

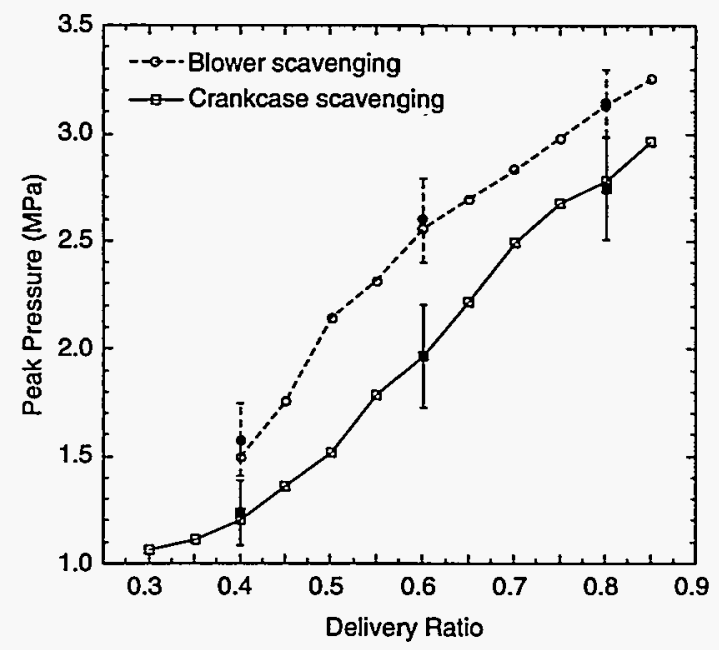

Figure 2 Comparison between blower- and crankcase-scavenged mean peak pressure as a function of delivery ratio.

which is characterized by lower RMS velocity fluctuations that are more spatially homogeneous.

Complementary in-cylinder pressure measurements are illustrated in Figure 2. These measurements indicate that, with external blower scavenging, combustion performance is characterized by a more rapid combustion process with higher peak pressures and less cyclic variation than is observed with crankcase scavenging. This may be due to: 1 ) better scavenging efficiency; 2) stratification of fresh mixture and residuals, such that more fresh mixture is present near the spark; or 3) higher levels of turbulence during the combustion process.

Additional details related to this work may be found in the references cited below.

This work was supported by the U.S. Department of Energy, Office of Advanced Industrial Concepts.

\section{References}

[1] Miles, P.C., Green, R.M., and Witze, P.O. "InCylinder Gas Velocity Measurements Comparing Crankcase and Blower Scavenging in a Fired TwoStroke Cycle Engine." SAE Paper 940401, 1994.

[2] Miles, P.C., Green, R.M., and Witze, P.O. "Comparison of In-Cylinder Scavenging Flows in a Two-Stroke Cycle Engine Under Motored and Fired Conditions." Proc. $7^{\text {th }}$ Int'l. Symp. on App. of Laser Tech. to Fluid Mech. Lisbon, Portugal. July 11-14, 1994. 


\title{
Diesel Engine Combustion Modeling using the Coherent Flame Model in Kiva-II
}

\author{
A flamelet model is used to calculate combustion in a diesel engine. The results are \\ compared to experimental data available from an optically accessible, \\ direct-injection diesel research engine.
}

\section{B. C. Dillies, ${ }^{1}$ K. D. Marx, J. E. Dec, and C. Espey²}

Research on diesel engines has recently seen an emphasis on both experimental work and numerical modeling in order to provide a better understanding of the processes involved in diesel combustion. In diesel engines, the flame structure is more complex and less wellknown than in spark-ignition engines because of the physical phenomena involved: the formation and evolution of spray droplets, fuel vaporization, auto-ignition of the gases, and propagation of the flame. Although threedimensional modeling of diesel combustion is not yet predictive, numerical simulation combined with experimental results can provide new insights into these issues.

This paper describes the recent development of the coherent flame model for diesel combustion and its application to an opticalaccess research engine. New features in the model include a four-step ignition delay and a method to account for the premixed burn in the early stages of combustion in the cycle.

Calculations were performed using the Kiva-II code (Amsden et al. [1]), in which the original Arrhenius combustion model was replaced by a four-step Arrhenius ignition model and a turbulent combustion flamelet model. In a flamelet model, the turbulent flame front is viewed as an ensemble of local interfaces, separating fuel from oxidizer in diffusion flames and premixed gases from burnt gases in premixed flames. This approach may be used when the Damköhler number is high, i.e., when the chemical times are much shorter than the turbulent mixing times. This hypoth-

'SAE Transactions 102, Sec. 3, pp. 96-117, SAE Paper 930074, 1993.

${ }^{1}$ Laboratoire EM2C du CNRS et de l'ECP, Chatenay-Malabry, France.

${ }^{2}$ Pennsylvania State University, State College, Pennsylvania. esis holds after ignition occurs in diesel combustion. The flamelet model used in the present work is an extension of the coherent flame model described by Marble and Broadwell [2], initially developed for turbulent diffusion combustion in a shear layer. The turbulent reaction zone is described as an ensemble of flamelet elements which are stretched by the turbulent flowfield, but which maintain their laminar flamelet structure locally. Briefly, this basic coherent flame description combines the following three elements (details may be found in Marble and Broadwell [2] and Veynante et al. [3]): (1) A model for the turbulent flow, including conservation equations and the k- $\varepsilon$ model for turbulence closure, (2) A local model of the strained laminar flame to calculate the laminar flame velocity using detailed chemistry and transport properties, and (3) A transport equation for the flame surface density, incorporating transport, diffusion, production and destruction of flame area.

To extend this model to diesel combustion, the physical ideas described above must be included while taking into account the following two diesel characteristics: (1) Ignition delay of the fuel jet (the basic flamelet model ignores this delay and assumes the flame is already turbulent and fully developed), and (2) The premixed and diffusion burns. After ignition, the premixed gases formed during the preignition phase burn very rapidly, showing the classical peak in the heat release, and combustion henceforth proceeds as a diffusion flame which is controlled by mixing and diffusion of the fuel in the air. In our model, these processes are simulated by introducing two flame surface densities into the calculations, one for the premixed burn and the other for the diffusion burn. 

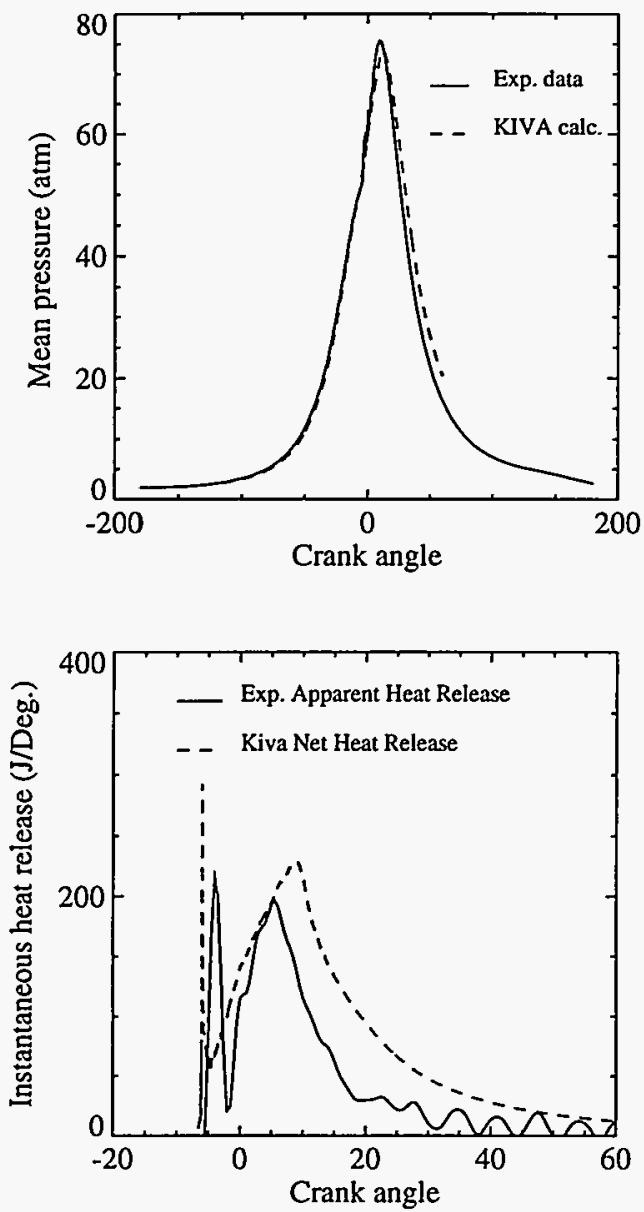

Figure 1. Comparison between calculated and experimental pressure, and calculated and experimental heat release. The engine speed is 1200 RPM and the equivalence ratio is $\mathbf{0 . 4 3}$.

The research engine is a single-cylinder, direct-injection diesel engine derived from a production Cummins $\mathrm{N}$-series six-cylinder engine with a bore of $140 \mathrm{~mm}$ and a stroke of $152 \mathrm{~mm}$. The compression ratio is 10:1. A closed-cup fuel injector is used in the experiment. A complete description of the experimental setup and technique is described in References [4-6].

Figure 1 shows results from a simulation of the engine when operating at 1200 RPM with an average equivalence ratio of 0.43 . The calculated ignition delay and peak pressure are seen to be quite close to that occurring in the experiment. The peak of the heat release curve which corresponds to the premixed burn is wider in the experiment than in the computation, indicating a need for improvement in either the vaporization model or the combustion model during this phase. The calculated heat release in the diffusion burn is somewhat greater than that observed experimentally; this is due to known inconsistencies in the model which are presently being corrected.

In conclusion, the combustion model developed in this work has proven to give satisfactory results at different operating conditions by modeling the physical phenomena involved in diesel ignition and combustion. In particular, the premixed and diffusion burns following ignition are taken into account by a flamelet model.

This work was supported by the Cummins Engine Company and the U.S. Department of Energy, Office of Industrial Technologies, Advanced Industrial Concepts Division.

\section{References}

[1] A. A. Amsden, P. J. O' Rourke and T. D. Butler, "Kiva-II : A Computer Program for Chemically Reactive Flows with Sprays", LA11560-MS

[2] F. E. Marble and J. E. Broadwell, "The coherent flame model for turbulent chemical reactions", Project Squid Rep. TRW - 9 - PU, 1977.

[3] D. Veynante, F. Lacas and S. M. Candel, "Numerical Simulation of the Transient Ignition Regime of a Turbulent Diffusion Flame," 27th Aerospace Science Meeting, Reno, Nevada, January 1989, AIAA paper 89-0487.

[4] J. Dec, "Soot distribution in a D.I. Diesel Engine Using 2-D Imaging of Laser-Induced Incandescence, Elastic Scattering, and Flame Luminosity," SAE Transactions, 101, Sec. 4, pp. 101-112, SAE paper 920115, 1992.

[5] J. E. Dec and C. Espey, "Soot and fuel distributions in a D.I. Diesel Engine via 2-D Imaging," SAE Transactions 101, Sec. 4, pp. 1642-1651, SAE paper $922307,1992$.

[6] C. Espey and J. E. Dec, "Diesel Engine Combustion Studies in a Newly Designed Optical-Access Engine Using High-Speed Visualization and 2-D Laser Imaging," SAE Transactions, 102, Sec. 4, pp. 703-723, SAE paper 930971, 1993. 


\section{Effects of Natural Gas Composition on Ignition Delay Under Diesel Conditions}

The effects of variations in natural gas composition on the autoignition of natural gas under diesel engine conditions have been examined experimentally. Typical variations in natural composition observed across the U.S. are shown to cause a shift in the ignition timing of up to six crank-angle-degrees under compression ignited conditions.

\section{J. D. Naber and D. L. Siebers}

Natural gas as a fuel for internal combustion engines offers an attractive combination of reduced emissions, low cost, and domestic availability. However, the chemical composition of commercial natural gas can vary significantly. For compression ignited diesel engine applications this composition variability could result in operational problems, since shock tube studies have shown that natural gas composition has a strong influence on its ignition characteristics.

This work examines the effects of natural gas composition on autoignition under diesel conditions, extending the previous research of Fraser et al. [1] to realistic natural gas compositions and engine conditions. Four natural gas compositions were considered, pure methane and the three blends listed in Table-1. The "mean" blend is an average natural gas delivered in the U.S., the "high ethane" blend is typical of Colorado fuels, and the "peak shaving" blend is representative of propane/air peak shaving practices.

Experiments to measure the ignition delay of the fuels in Table 1 were performed in a constant volume combustion vessel over a range of ambient pressures and temperatures. This range included the top-dead-center (TDC) compression conditions in the 6V-92 natural gas diesel engine (1150 K and 6.8 MPa) under development at Detroit Diesel Corporation. The ambient pressures and temperatures in the vessel were generated by first spark igniting and burning a lean premixed charge of gaseous fuels, and then waiting for the combustion products to cool due to heat transfer to the chamber walls $\left(\mathrm{T}_{\mathrm{w}}=180^{\circ} \mathrm{C}\right)$. At a preselected pressure-temp-erature state dur- ing the cool down, the fuel injector was activated, and the ensuing fuel jet autoignited and burned as in a diesel engine. The lean premixed charge was composed of $\mathrm{H}_{2} / \mathrm{C}_{2} \mathrm{H}_{2} / \mathrm{O}_{2} / \mathrm{N}_{2}$ and was tailored so that the combustion products would have properties similar to air (e.g., $21 \% \mathrm{O}_{2}$ and a molecular weight of 29.5).

Table 1. Selected natural gas mixtures.

\begin{tabular}{|l|c|c|c|}
\hline Species & $\begin{array}{c}\text { Mean } \\
(\% \text { Vol })\end{array}$ & $\begin{array}{c}\text { High } \\
\text { Ethane } \\
(\% \text { Vol })\end{array}$ & $\begin{array}{c}\text { Peak } \\
\text { Shaving } \\
(\% \text { Vol })\end{array}$ \\
\hline $\mathrm{N}_{2}$ & 1.37 & 7.76 & 4.34 \\
\hline $\mathrm{CO}_{2}$ & 1.21 & 1.43 & 1.13 \\
\hline $\mathrm{CH}_{4}$ & 93.12 & 80.56 & 87.15 \\
\hline $\mathrm{C}_{2} \mathrm{H}_{6}$ & 3.20 & 8.99 & 3.00 \\
\hline $\mathrm{C}_{3} \mathrm{H}_{8}$ & 0.70 & 1.00 & 4.01 \\
\hline $\mathrm{C}_{4} \mathrm{H}_{10}$ & 0.40 & 0.26 & 0.37 \\
\hline
\end{tabular}

The natural gas injector used produced a "top hat" injection rate profile with very rapid opening and closing times. The results reported here are for a single orifice nozzle with a $250 \mu \mathrm{m}$ diameter orifice and a 4.1 length/diameter ratio, an injection pressure of $20.7 \mathrm{MPa}$, a fuel temperature of $450 \mathrm{~K}$, and an injection duration of $12 \mathrm{~ms}$.

The measured "mean" natural gas ignition delays are plotted versus ambient temperature at the time of ignition in Fig. 1. The ignition delay $(\tau)$ is defined as the time from the start of injection until the pressure rises $14 \mathrm{kPa}$. Also shown in the figure is the least-squares fit of the ignition delay data to: 


$$
r=\left\{C^{2}+\left[A e^{E_{a} / R T}\right]^{2}\right\}^{1 / 2}
$$

Equation 1 is the root-mean-square sum of an Arrhenius term, $A \cdot \exp \left(E_{\mathrm{a}} / \mathrm{RT}\right)$, and a constant physical delay, $C$.

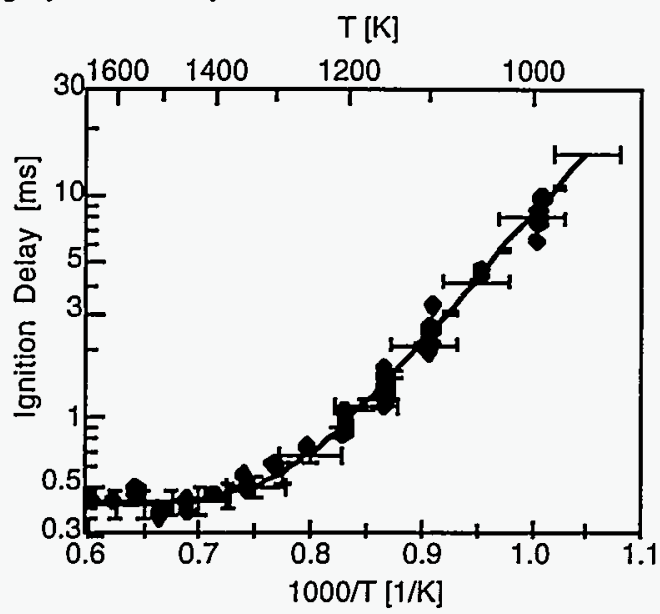

Figure 1. Measured ignition delay (diamonds) for the mean natural gas fuel blend at an ambient density of $20.4 \mathrm{~kg} / \mathrm{m}^{3}$. The vertical bars $( \pm 60 \mathrm{~ms})$ are the uncertainty in the time measurement. The horizontal bars are the measured rms temperature fluctuations.

The general trends noted in Fig. 1 are similar for each of the four compositions considered. The effect of temperature is considerable over the temperature range most relevant to DI diesel operation (1000-1300 K). The measured ignition delay in this range changes by an order of magnitude with a $20 \%$ change in $1 / \mathrm{T}$. At high temperatures ( $>1400 \mathrm{~K})$, the ignition delay reaches a minimum of $0.4 \mathrm{~ms}$ due to limiting physical processes. The minimum temperature required for a reasonable diesel engine ignition delay ( $<2 \mathrm{~ms}$ ) for the four natural gas blends is between 1100 and $1150 \mathrm{~K}$, slightly lower than the temperatures reported by Fraser et al. [1] which were based on data obtained at lower ambient densities for binary methane/ethane mixtures.

The fits of the data for all four natural gas blends to Eq. 1 are shown in Fig. 2. In general, addition of ethane, propane, and n-butane to pure methane decreases the ignition delay over this temperature range. The high ethane and peak shaving natural gases, which have the largest percentages of higher hydrocarbons, have the shortest ignition times. These trends are in agreement with results at lower pressures and are due to differences in the kinetic chain branching processes between methane and the higher hydrocarbon fuels. Further discussion of these points and comparisons to predictions with a detailed kinetics model can be found in the Ref. [2].

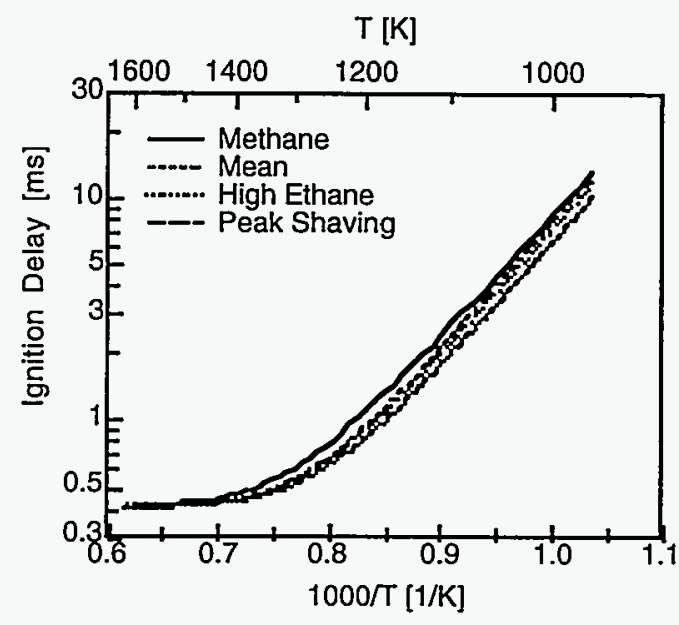

Figure 2. Comparison of the ignition delays at an ambient density of $20.4 \mathrm{~kg} / \mathrm{m}^{3}$.

The results show that for expected TDC temperatures in the 6V-92 engine (1100 to 1150 $\mathrm{K})$, the ignition delay can vary by as much as 0.5 to $0.7 \mathrm{~ms}$ over the range of fuel compositions considered. This change in ignition delay corresponds to a six crank-angle-degree shift in ignition timing at an engine speed of 1500 RPM. A shift in ignition timing of this order is sufficient to alter the rates of pressurerise and the fraction of premixed versus mixing-controlled combustion, which in turn can produce performance and emissions changes.

This work was supported by the Gas Research Institute.

\section{References}

[1] Fraser, R.A., Siebers, D.L., and Edwards, C.F., "Autoignition of Methane and Natural Gas in a Simulated Diesel Environment," Transactions of the SAE, Vol. 100, pp. 33-45, 1991.

[2] Naber, J.D., Siebers, D.L., Caton, J.A., Westbrook, C.K., and Di Julio, S.S. “Natural Gas Autoignition Under Diesel Conditions: Experiments and Chemical Kinetic Modeling," SAE paper 942034, 1994. 


\title{
Characterization of the LDV Fringe Field
}

\author{
A technique to measure the fringe field in the probe volume of a dual beam laser Doppler \\ velocimeter is developed, which provides a sensitive measure of instrument alignment and \\ allows quantitative determination of measurement precision. The fringe field is further \\ examined analytically to elucidate the system parameters which govern fringe uniformity.

\section{P. C. Miles and P. O. Witze}

Since the introduction of laser Doppler velocimetry (LDV) approximately thirty years ago, it has become the preferred technique for obtaining quantitative, non-intrusive measurements of fluid velocity. As optical systems, signal processors, and data reduction algorithms have evolved, the accuracy and precision of LDV measurements has improved. Today, one of the dominant sources of error is a fundamental physical limitation associated with non-planar wavefronts of the intersecting laser beams, manifested as fringe field nonuniformity in the probe volume. Accurate knowledge of the fringe geometry allows the minimization of this error source through precise instrument alignment, and quantification of its contribution to the measured velocities. By understanding the system parameters which govern this source of error, the LDV system designer can take additional steps to minimize its effect.

In the upper portion of Fig. 1 the geometry of the standard dual beam LDV configuration is depicted. Two parallel laser beams are crossed and focused by a single transmitting lens. Ideally, each beam is focused to a minimum diameter (waist) at the crossing (probe volume). The signal frequency content can be modeled as being generated by passage of a particle through a set of interference fringes in the probe volume (inset of Fig. 1), with the frequency given by the particle velocity normal to the fringes divided by the distance between the fringes.

The fringes are surfaces of fixed phase difference between the two beams. If the wavefronts of the two beams were planar throughout the probe volume, then the distance $d_{f}$ between fringes would be uniform. However, because the wavefronts have finite curvature which evolves and changes sign as the beam passes through the waist, the fringes in the probe volume are not planar, parallel surfaces. Accurate measurement of the geometry of these surfaces is complicated by the small distance between them; typically $d_{f}$ is about $1-$ $5 \mu \mathrm{m}$. Measurement of the fringe geometry can be simplified considerably, however, if a magnified image of the fringe field is created.

In the lower portion of Fig. 1 an imaging lens is placed just below the probe volume. Each beam, after passing through the probe volume, strikes the imaging lens and is transformed to a beam with a much smaller waist

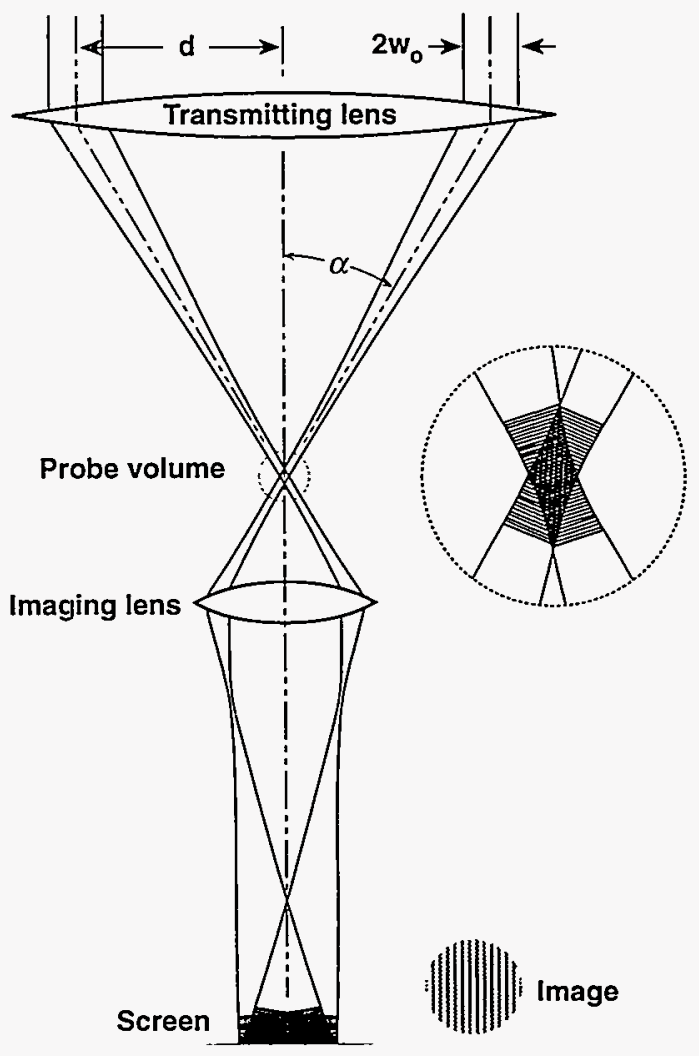

Figure 1 Standard dual beam LDV geometry and fringe field imaging set-up. 
and larger divergence. The transformed beams propagate and expand, until they cross and form interference fringes on a far screen. From a classical imaging viewpoint, the fringe field at the image screen may be considered to be an inverted, magnified replica of the fringe field at the corresponding object plane. The fringe geometry, however, is determined by the relative phase between the beams-which, in turn, is influenced by wavefront curvature. Because the beam curvature and evolution are very different at the probe volume and at the image screen, one must question whether the relative phase relation between the two beams is preserved, and whether the classical imaging view is appropriate.

By performing a diffraction analysis which tracks the phase of each beam on passage through the imaging lens, and subsequently interfering the beams at the image screen, we have confirmed the classical imaging relationship for Gaussian beams. Furthermore, we have demonstrated that this relationship can be readily achieved in the laboratory. Figure 2 shows the fringe fields measured in the probe volume and at the image screen using a scanning pinhole and photodiode combination. By varying the effective object plane at fixed magnification, it is possible to obtain a mapping of the entire probe volume fringe field. Comparison of the measured fringe fields clearly demonstrates that the imaging technique can be quantitatively applied.

The above mentioned diffraction analysis allows several additional conclusions to be reached. First, variation in fringe spacing in the direction perpendicular to the plane of Fig. 2 is found to be small, and beam phase variations along this direction are thus not critical to obtaining precise measurements. Second, an analytical expression for the variation in fringe spacing can be obtained for conditions of ideal alignment. In this case,

$$
d_{f}=\frac{\lambda}{2 \sin \alpha}\left(1+\left(\frac{\hat{z} w_{0}}{d}\right)^{2}\right)
$$

where $\hat{z}$ is a longitudinal coordinate normalized by the probe volume length $(|\hat{z}| \leq 1)$. Eq. 1 demonstrates that the variation in fringe spacing within the probe volume is solely determined by the ratio of the laser beam radius prior to the transmitting lens and the spacing between the beams. These are variables which
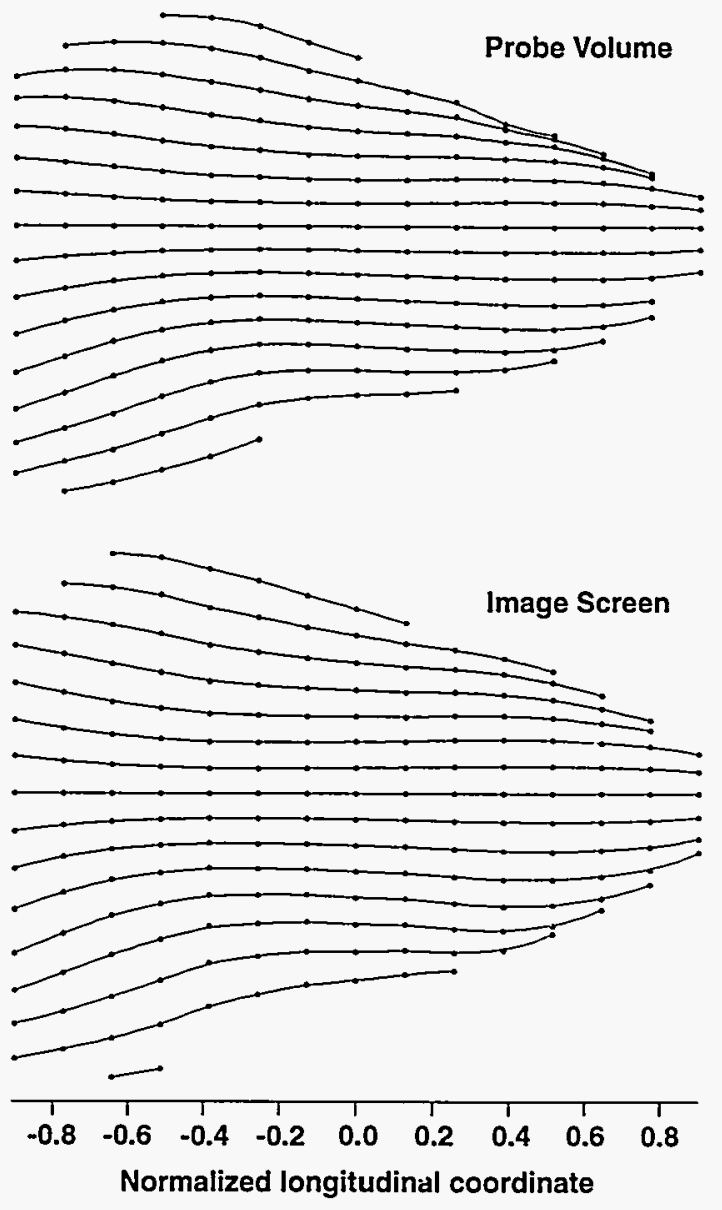

Figure 2 Fringe fields measured in the LDV probe volume and in a magnified image. The measured variation has been exaggerated by a factor of 20 .

the LDV system designer can use to tailor the instrument to a specific application.

Finally, the measured fringe fields reveal a significant and complex variation in the fringe spacing along both the length and width of the probe volume. These variations can not be reproduced using a Gaussian beam model, and our current efforts are concentrated on understanding the cause of this behavior. Additional details related to this work are given in the reference cited below.

This work was supported by the U.S. Department of Energy, Office of Advanced Industrial Concepts.

\section{References}

[1] Miles, P.C. and Witze, P.O. "Fringe Field Quantification in an LDV Probe Volume by Use of a Magnified Image." Exp. in Fluids, 16, 330-335, 1994. 


\title{
Research and Development on Advanced Gas-Fired Burners in BERL
}

\author{
The Burner Engineering Research Laboratory has completed three burner tests. The first was \\ a study of a $300 \mathrm{~kW}$ research burner as part of an examination of burner scaling criteria. The \\ other two tests were investigations of new industrial burner concepts.
}

\section{N. Fornaciari, R. Sanford ${ }^{1}$ and L. Claytor ${ }^{1}$}

The Burner Engineering Research Laboratory (BERL) is a facility where advanced diagnostics are used to help natural gas burner manufacturers understand the physics governing combustion and pollutant formation in their burners. Since its initial firing in October 1992, BERL has hosted three burner tests.

The first test in BERL was on a $300 \mathrm{~kW}$ version of the burner used in the International Flame Research Foundation's (IFRF) SCALING 400 study. The SCALING 400 study consisted of testing a generic low-NOx burner design at 30 and $300 \mathrm{~kW}$ and 1,4 , and $12 \mathrm{MW}$ under similar confinement and boundary conditions. The data gathered during these tests is being used to develop empirical correlations and a computer model to assist burner designers with scaling (i.e. building a 10 or $20 \mathrm{MW}$ version of a burner which has identical emissions characteristics of a $1 \mathrm{MW}$ version). The BERL test provided the IFRF with a detailed data set on the $300 \mathrm{~kW}$ burner at the same operating conditions, under different confinement and boundary conditions. This data will be used to test the correlations and model predictions. Both conventional and laser diagnostics were used to gather the data. In-furnace probes were used to measure temperature, radiative flux to the wall, and gas composition. Laser Doppler velocimetry was employed to measure the gas velocities in the furnace and planar Mie scattering was used to visualize the mixing of fuel, air and products.

The second test in BERL was on the StAR II burner developed by Arthur D Little, Massachusetts Institute of Technology and

\footnotetext{
'Energy and Environmental Research Corporation
}

Hauck Manufacturing. The StAR burner was designed to produce low NOx emissions in high temperature processes (e.g. heat treating, forging, and glass and aluminum melting) and uses air staging and flue gas recirculation to achieve this goal. The objective of this test was to optimize the current design and gain insight for design modifications on the current version and new designs for other operating environments. In addition to the diagnostics used in the IFRF test, $\mathrm{CH}$ chemiluminescence and fluorescence were used to observe combustion. These diagnostics illuminated an area of high NOx formation (see Figure 1) and a strategy was identified to modify the burner and eliminate this region. The optimization part of the test yielded a $20 \%$ reduction in NOx output from the current burner and several modifications were tested that gave the engineers ideas for the design of two new versions of the StAR concept.

The third test in BERL was of the Acurex $\mathrm{Hi}-\mathrm{Rad}$ concept. The Hi-Rad is a unique concept that greatly increases the luminosity of natural gas flames by passing some of the natural gas through a plasma that "cracks" the gas into carbon particles. These particles are convected into the flame, increasing the luminosity to nearly that of an oil flame. This increased luminosity would facilitate the use of cleaner burning natural gas in processes that benefit from high radiative heat transfer from the flame. The goals of this test were to characterize the radiative flux to the walls and the carbon particle size and distribution in the flame at several different operating conditions. Ellipsoidal and narrow angle radiometers were used to measure the radiative flux at the walls and in the flame respectively. Scattering and extinction measurements were made to 
determine the carbon particle size and distribution.

These first three tests underscored the utility of BERL in providing valuable insight into burner operation to scientists and designers.

This work was supported by the Gas Research Institute and the U.S. Department of Energy, Office of Industrial Technologies, Industrial Energy Efficiency Division and the Office of Basic Energy Sciences, Division of Chemical Sciences.

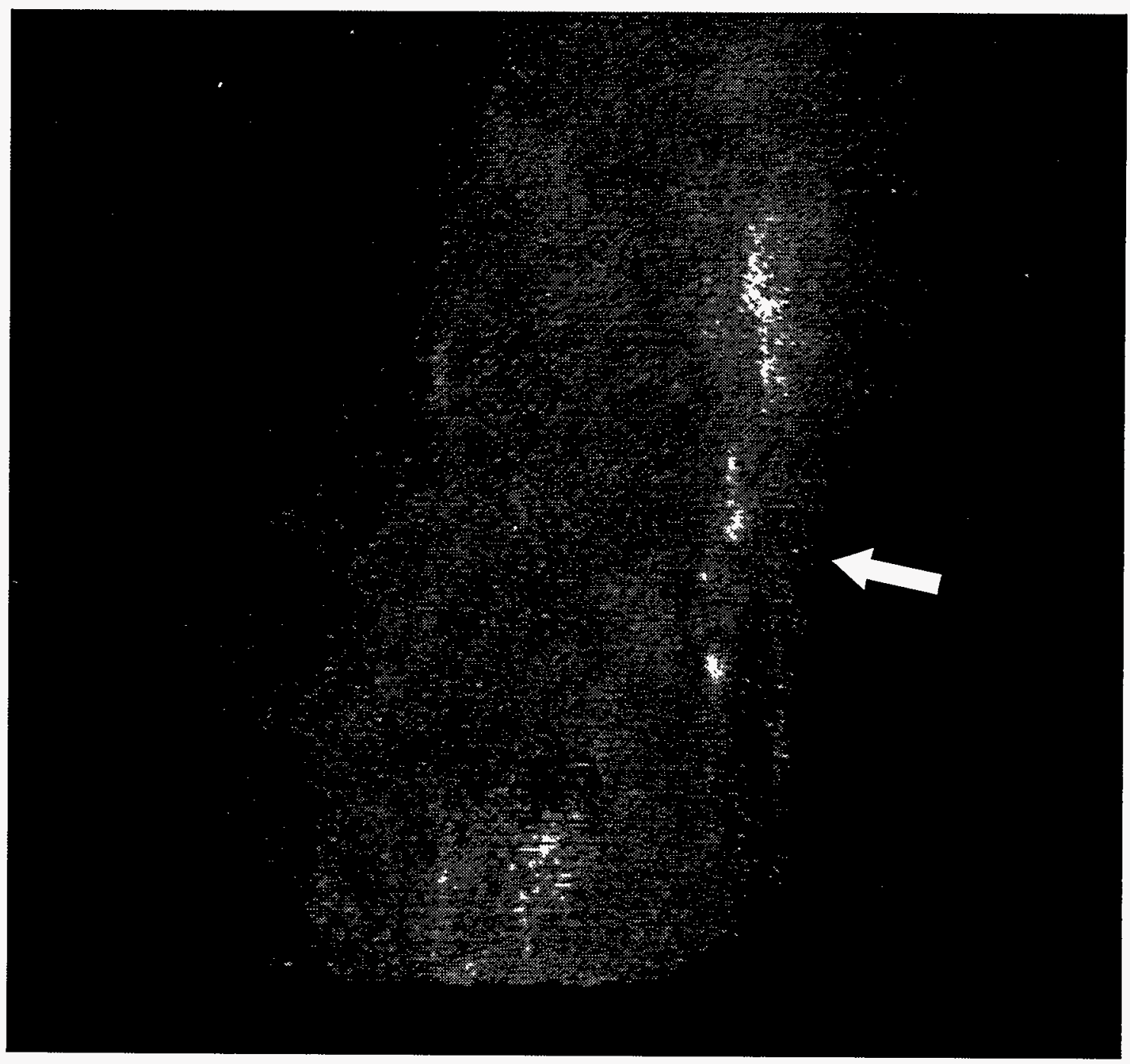

Figure 1. CH chemiluminescence image of the flame from the StAR II burner. Arrow points to location of high NOx production. 


\title{
Statistical Structure of the Ideal Spray*
}

\author{
A theoretical framework is developed for an ideal representation of sprays. It is shown that \\ useful qualitative and quantitative information can be extracted from the experimentally \\ measured interparticle time distribution. An example is given of the determination of spray \\ unsteadiness and of the statistical moments of the intensity function of a spray.
}

\section{F. Edwards and K. D. Marx}

In this study we develop the theoretical framework required for analysis of the timebased multi-point statistics of sprays. This is accomplished in the context of the ideal spray-a random assemblage of droplets modeled as non-interacting point particles. It is shown that such a spray may be decomposed into a series of independent single-class sprays, each of which is driven by an inhomogeneous temporal Poisson process. (The term "class" is used to denote a collection of particles in, e.g., a given size and velocity range.) Complete spray behavior is found by superposition of these Poisson processes. A function referred to as the realization density can be derived which contains all possible information about one of these single-class sprays $[1,2]$. All of the customary multi-point statistics-the autocorrelation, power spectral density, fluctuation moments, etc.-may be developed from the realization density by suitable integrations over its probability space.

These concepts can be utilized to evaluate the steadiness of sprays. This is achieved through investigation of the interparticle time distribution $h(\tau)$, which is the distribution of times between particle counts for the particle class. This distribution is easily determined from (e.g.) phase Doppler measurements and contains both qualitative and quantitative information about the expected occurrence rate of droplets in a spray. The qualitative information may be extracted by comparison of an experimentally measured histogram with theoretical expressions developed for the steady ideal spray. Quantitative information may be obtained for any spray with stationary statis-

\footnotetext{
"Proceedings of the Sixth Annual Conference on Liquid Atomization and Spray Systems, ILASS 93, Worcester, Massachusetts, May 1993.
}

tics in the form of the first few moments of those statistics. These values are obtained by determination of the zero delay limit of the interparticle time distribution and its derivative. In this paper, we derive theoretical expressions for the interparticle time distribution in the case of steady ideal sprays. We also derive results showing that the moments of the driving intensity function of a fluctuating spray may be found from its time-zero value and derivative.

For a given class of particles detected by a measurement system, the intensity function $\lambda(t)$ which characterizes the class is defined such that the probability that a particle is counted in an interval $d t$ at time $t$ is $\lambda(t) d t$. For any experiment, there is a probability distribution $p(\lambda)$ which gives the probability that the intensity has the value $\lambda$. The statistical moments referred to above are the moments of $p(\lambda)$, i.e., $\langle\lambda\rangle,\left\langle\lambda^{2}\right\rangle,\left\langle\lambda^{3}\right\rangle$, etc. These moments also offer a characterization of the spray; in principle, $p(\lambda)$ can be determined from knowledge of all its moments. However, neither $\lambda(t)$ nor $p(\lambda)$ can be measured directly in an experiment.

Applying Poisson statistics to the particle counts, it is possible to derive relationships between the measured interparticle time distribution $h(\tau)$ and the moments of $p(\lambda)$. For example, in the limit that data is taken for an infinitely long time, $h(0)=\left\langle\lambda^{2}\right\rangle /\langle\lambda\rangle$ and $h(' 0)=$ $-<\lambda^{3}>/<\lambda>$, where $h(0)$ and $h\left({ }^{\prime} 0\right)$ are obtained from the behavior of the $h(\tau)$ distribution as $\tau$ goes to 0 . Since the mean value $\langle\lambda\rangle$ is obtained from the total number of counts, the second and third moments $\left\langle\lambda^{2}\right\rangle$ and $\left\langle\lambda^{3}\right\rangle$ can be extracted from the measurement of $h(\tau)$.

We now illustrate that these intensity moments may be correctly obtained from use 
of the zero-delay limit of the interparticle time distribution by applying the analysis to synthetic spray data with known fluctuation characteristics. The synthetic spray used for the purpose varied sinusoidally in occurrence rate with a time-averaged intensity $\langle\lambda\rangle$ of 100 $\mathrm{s}^{-1}$ and a root-mean-square fluctuation $\lambda_{r m s}$ of $50 \mathrm{~s}^{-1}$. The frequency of the fluctuation used in the simulation was $10 \mathrm{~s}^{-1}$, but the relevant statistical data is independent of the frequency. For this function, it is possible to derive a closed-form expression for $p(\lambda)$ and obtain the exact values of its moments.

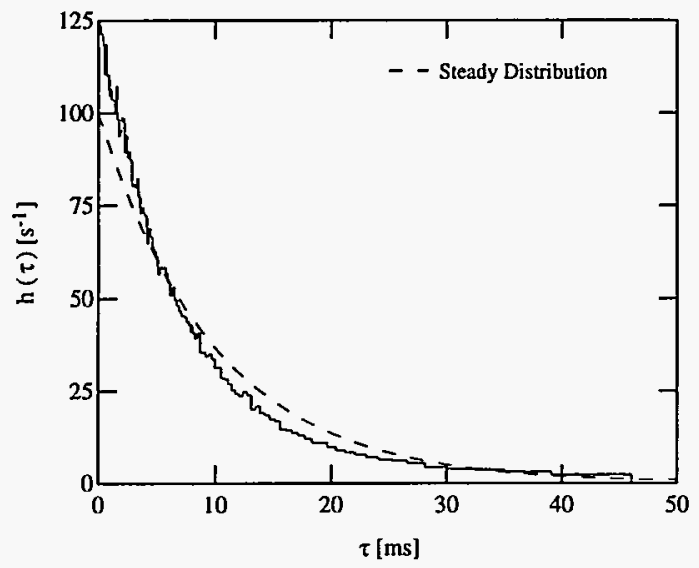

Figure 1. Comparison of the interparticle time histogram from a simulated unsteady singleclass spray with that from a steady theoretical distribution with the same mean value of intensity $<\lambda>$.

Figure 1 shows the interparticle time histogram generated from a single realization 1000 seconds in duration, so that a total of $\sim 100,000$ events were obtained. The "measured" unsteady distribution is more skewed toward the short interparticle times than the corresponding steady distribution, which indicates that the intensity has a nonzero RMS fluctuation, i.e., that the simulated spray is unsteady.

Table I lists the exact values of the intensity moments along with those determined from the simulation by using the procedures developed in this work. The $h^{\prime}(0)$ value was found by applying a linear fit to the first $2 \mathrm{~ms}$ of histogram data in the figure.

The results shown in the table demonstrate that the procedures developed here are capable of accurately reproducing the intensity moments of the synthetic spray, albeit with the uncertainty in those determinations increasing with the order of the moment sought.

The above illustration is one example of the use of spray measurement statistics to characterize a spray. These concepts can be extended to a classification of sprays into three major categories: steady, unsteady/deterministic, and unsteady/stochastic. This categorization may be further refined into subcategories such as sinusoidal, complex periodic, almost periodic, transient, strictly stationary, $\mathrm{N}$ point stationary, weakly stationary, and nonstationary. Methods for using time series phase Doppler data to classify sprays into these categories have been developed [3].

This work was supported by the U.S. Department of Energy, Office of Industrial Technologies, Advanced Industrial Concepts Division.

Table 1. Comparison of exact and simulationderived intensity moments for a sinusoidal synthetic spray.

\begin{tabular}{|c|c|c|c|}
\hline $\begin{array}{c}\text { Mom- } \\
\text { ent }\end{array}$ & $\begin{array}{c}\text { Exact } \\
\text { Value }\end{array}$ & Simulation & $\%$ Diff. \\
\hline$<\lambda>$ & $100 \mathrm{~s}^{-1}$ & $100.08 \mathrm{~s}^{-1}$ & $+0.08 \%$ \\
$<\lambda^{2}>$ & $12,500 \mathrm{~s}^{-2}$ & $12,900 \mathrm{~s}^{-2}$ & $+3.2 \%$ \\
$<\lambda^{3}>$ & $1.75 \times 10^{6} \mathrm{~s}^{-3}$ & $1.57 \times 10^{6} \mathrm{~s}^{-3}$ & $-10.3 \%$ \\
\hline
\end{tabular}

\section{References}

[1] D. L. Snyder and M. I. Miller, Random Point Processes in Time and Space, Springer-Verlag, New York, 1991.

[2] C. F. Edwards and K. D. Marx, "Multi-Point Statistical Structure of the Ideal Spray. Part I: Fundamental Concepts and the Realization Density," accepted for publication in Atomization and Sprays, 1994.

[3] C. F. Edwards and K. D. Marx, "Multi-Point Statistical Structure of the Ideal Spray. Part II: Evaluating Steadiness Using the Interparticle Time Distribution," accepted for publication in Atomization and Sprays, 1994. 


\title{
SPCDC: A User-Friendly Computer Code for the Design of Practical Pulse Combustion Systems
}

\begin{abstract}
A user-friendly PC-executable computer code is being developed to assist engineers both in designing pulse combustors for specific applications and in refining existing units. This code, named the Sandia Pulse Combustor Design Code, or SPCDC, represents the culmination of over ten years of research and development in the field of pulse combustion.
\end{abstract}

\section{P. K. Barr, J. O. Keller and J. A. Kezerle1}

Pulse combustors use a periodic combustion process to drive a resonant pressure wave. The design under consideration here is known as a Helmholtz-type pulse combustor, shown in Figure 1. It consists of a closed cylinder, which acts as the combustion chamber, that is attached to a long tail pipe through a transition section. Fresh charge is introduced into the combustion chamber through either a flapper valve or an aerodynamic valve. Both types of valves allow the gas to flow in one direction; the flow in the other direction is blocked by a mechanical flapper in the flapper valve, and it is strongly resisted by the geometry of the aerodynamic valve. The exhaust decoupler shown in the figure is not needed to support the oscillations, rather it is used in commercially available systems to terminate the acoustic wave, suppressing sound emission.

The advantages of pulse combustors are numerous. The heat transfer is enhanced by the large oscillations in the flow field. Heat transfer rates in pulse combustors have been measured that are up to two and a half times greater than those obtained for steady turbulent flows at the same mean Reynolds numbers. The enhanced heat transfer means that a smaller furnace can be used to provide the same energy output. Also, pulse combustors do not require expensive auxiliary equipment such as external blowers or flues. The pressure oscillations enable them to draw in their own supply of fuel and air, and the resulting thrust is used to vent the exhaust products through a small diameter plastic pipe, which allows more flexibility in siting the unit. The maintenance expenses are low because there are few

\footnotetext{
${ }^{1}$ Gas Research Institute, Chicago, Illinois.
}

movable parts. Another advantage of gas-fired pulse combustors is the low $\mathrm{NO}_{\mathrm{x}}$ level in the exhaust gas obtained without additional pollution control equipment. $\mathrm{NO}_{x}$ emissions as low as 3 ppm have been measured from practical size units. The main disadvantages of pulse combustors are caused by the large oscillations, which result in noise and vibrational issues. However, the combustor's physical structure can be designed to reduce these problems.

The Sandia Pulse Combustor Design Code, or SPCDC, has grown out of knowlege gained from over ten years of research and development funded by the DOE, GRI, and others. SPCDC couples both the fuel-air injection and the energy release to the time-varying pressure wave. The pressure distribution throughout the unit is determined from the wave dynamics of the system, which are computed using a finite-difference method to solve the nonlinear equations of continuity, momentum and energy. Because the injection and combustion processes both drive and are driven by the wave dynamics, this model couples the major processes that occur in a pulse combustor.

Currently, SPCDC is being used by engineers from several U.S. manufacturers to design pulse combustors for fluid heating applications. With this model they have been able to recreate much of the experimental data obtained from their pulse combustors, including the influence of system geometry, heat transfer, reactant supply pressure, as well as other combustor operating conditions. With SPCDC they can optimize a design for specific 


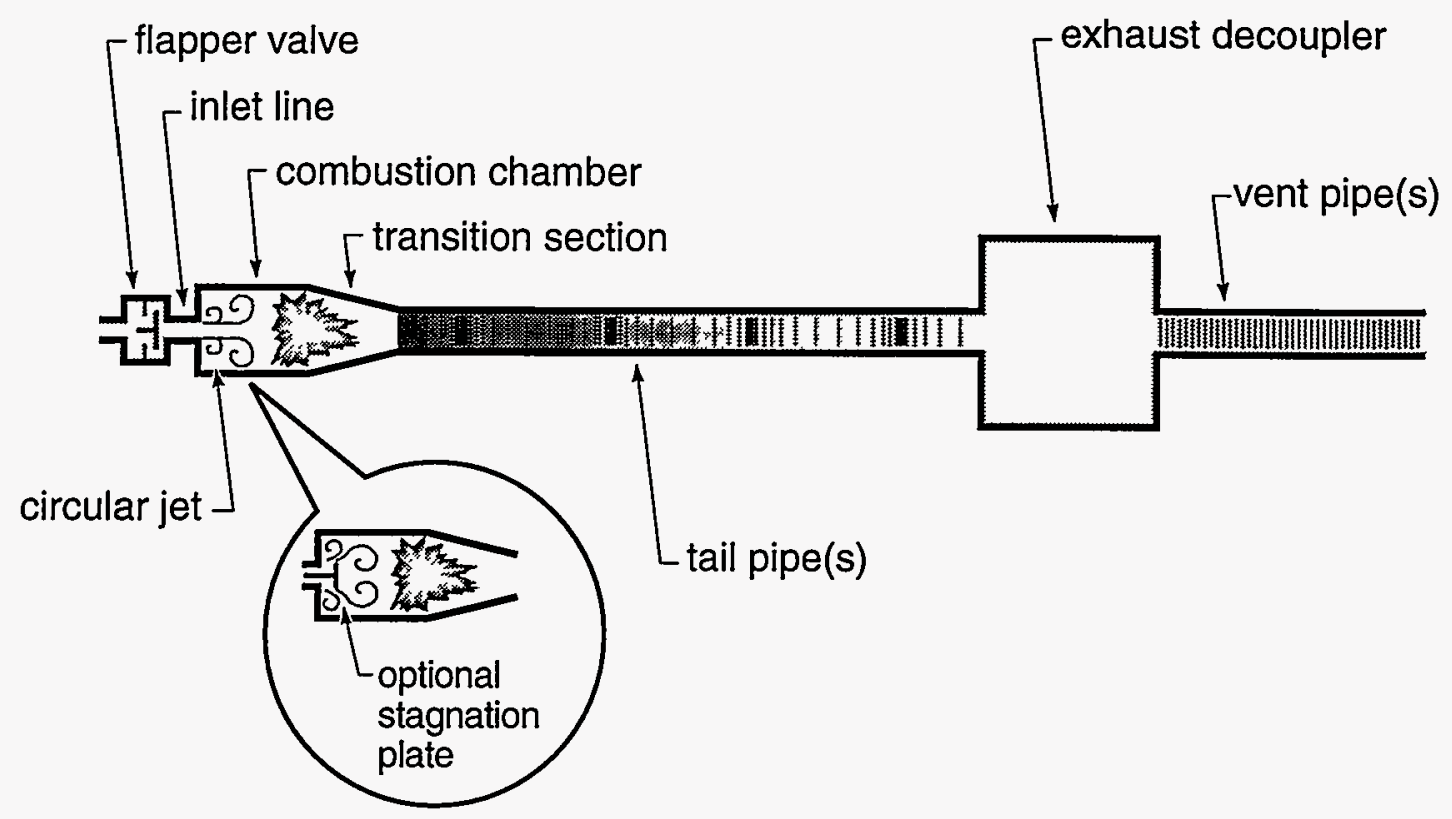

Figure 1. Schematic of a Helmholtz-type pulse combustor, showing the dominant processes that occur in each region, which are included in SPCDC.

applications, or to scale up a design to a desired input power rate.

There are several different pulse combustor units that are currently being marketed for fluid heating applications by Lennox, Hydrotherm and Fulton, but because of the highly coupled interactions of the controlling mechanisms, pulse combustion systems are difficult to design and scale. Design handbooks provide a systematic approach to the design of a Helmholtz-type pulse combustor for a particular application. However, handbooks do not allow one to investigate the stability of a particular design, the difficulties associated with scaling a particular design, or the implications of installing a pulse combustor in an area that has an unusual fuel composition.

SPCDC is being developed with guidance from engineers representing companies that manufacture pulse combustion systems. Its predictions have been compared against data from commercial and prototype hardware. It draws upon many aspects of pulse combustion research and development, including fundamental experimental research, multidimensional computations using supercomputers, and extensive development for specific applications. SPCDC has evolved from a re- search tool developed to help understand the coupling between the acoustics, the injection and the energy release for a bare bones combustor configuration into a user-friendly engineering tool. Many algorithms have been added to the research code to make SPCDC useful to pulse combustor manufacturers.

SPCDC simulates an entire pulse combustion system, from the intake line to the exhaust decoupler, as shown in Figure 1. It can be used to compare sound power levels emitted from pulse combustors running at different operating conditions. It can investigate the impact of the intake line lengths required for different installations. It can explore the feasibility of input power turn-down options. Although SPCDC will never replace the time-proven method of actually building and testing a unit, it can significantly reduce the number of units that must be tested. And, it can help produce a superior pulse combustion system tailored to a specific application.

This work is supported by the Gas Research Institute. 


\title{
$\mathrm{NO}_{\mathrm{x}}$ and CO Emissions from a Pulse Combustor Operating in a Lean Premixed Mode
}

\author{
Control over the combustion fluid dynamics was used to minimize the emission of $N O_{x}$ and \\ CO. Emission levels below 5.0 ppm $\mathrm{NO}_{x}$, with corresponding levels of $5.0 \mathrm{ppm} \mathrm{CO}$ \\ (corrected to $3 \% \mathrm{O}_{2}$ ), were achieved in a pulse combustor operating in a lean premixed mode, \\ without the use of any post-combustion clean-up technologies.
}

\section{J. O. Keller, T. T. Bramlette, P. K. Barr, and J. R. Alvarez}

Pulse combustors are known to have relatively low $\mathrm{NO}_{\mathrm{x}}$ emission (34-46 ppm compared to $58-138 \mathrm{ppm}$ for conventional boilers [1]). ${ }^{1}$ However, current regulations require $\mathrm{NO}_{x}$ emission levels below those produced from current pulse combustor designs. ${ }^{2}$

Pulse combustion has many inherent features that make the realization of ultra-low $\mathrm{NO}_{x}$ emissions possible. The pulse combustor is one example of an acoustically modified combustion process where a periodic injection causes reactants to mix with residual combustion products and subsequently ignite. When the injection and combustion processes are suitably tuned to the acoustics, a flow in acoustic resonance may develop, as is the case with pulse combustion. The mechanism responsible for the inherently low $\mathrm{NO}_{\mathrm{x}}$ emission levels from pulse combustors has been shown to be a short residence time at high temperature caused by rapid mixing with cooler residual gases [3]. Other work on the fundamental mixing and combustion characteristics of pulse combustion has shown that the reactants mix with the residual products in a highly strained flow field, preparing the reactants for combustion in an almost well-stirred manner $[4,5]$. It has also been shown that this mixing and subsequent combustion process are readily controllable [69].

Control of the combustion was obtained by operating the combustor lean premixed,

*Comibustion and Flame (1995), in press.

${ }^{2}$ The most stringent regulations are approaching 20 ppm corrected to $3 \% \mathrm{O}_{2}$ [2]. and by controlling the rate and direction of vorticity deposition into the combustion chamber thus controlling the natural cascade of the toroidal vortex to fine-scale threedimensional motion. Controlling the fluid dynamic microscopic mixing characteristics through the vorticity field provides control over the residence time under high NO production conditions. Three different injection systems were used to control the vorticity deposition: 1) a smooth stagnation plate was used as the base case to create a large scale phase locked toroidal vortex, 2) a starred plate was substituted for the smooth plate to enhance the natural cascade of the large scale vortical structure to fine scale[9], and 3) a swirl generator was used in conjunction with the smooth plate to create a streamwise vorticity component that also promotes the breakdown from large scale to fine scale. The results of this work are shown in Figure 1. The decrease in thermal NO levels with an increase in the microscopic mixing was not detected in these measurements, as shown in the figure. The expected decrease in NO production due to an increase in fine scale mixing (resulting in a decrease in residence time) was on the order of $10 \%$ which is within the scatter of the data presented here. The NO emission at very low combustion temperatures (effectively turning off the thermal mechanism) was found to be invariant with changes in the microscopic mixing rates obtained in this work. Since the peak concentrations of $\mathrm{CH}$ was previously found to be invariant with these changes in the microscopic mixing rates [9], the production of $\mathrm{NO}$ by either the $\mathrm{HCN}$ or the $\mathrm{N}_{2} \mathrm{O}$ mechanisms is assumed to be invariant with 


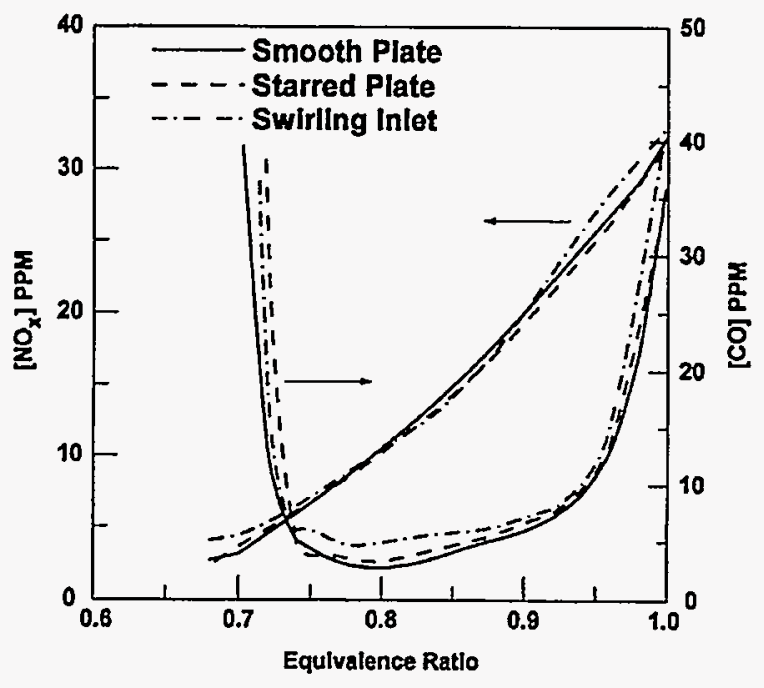

Figure 1. Comparison between different microscopic mixing characteristics of $\mathrm{NO}_{\mathbf{x}}$ and $\mathrm{CO}$ measurements corrected to $3 \% \mathrm{O}_{2}$

these changes in microscopic mixing. Indeed, it is likely that the NO production at these levels is limited by either the $\mathrm{HCN}$ or the $\mathrm{N}_{2} \mathrm{O}$ mechanisms, or both.

By designing and controlling the combustion process to minimize emission levels, ultra-low values of $\mathrm{NO}_{\mathrm{x}}$ and $\mathrm{NO}$ (as low as $2.9 \mathrm{ppm}$ and $2.8 \mathrm{ppm}$, respectively) have been achieved without the use of any post combustion clean-up technologies. Operating the combustor at a sufficiently high equivalence ratio to force $\mathrm{CO}$ burn-out $(\phi=$ 0.74 ) results in emission levels of $2.9 \mathrm{ppm}$ of $\mathrm{CO}$ and 5.2 and $5.0 \mathrm{ppm}$ of $\mathrm{NO}_{x}$ and $\mathrm{NO}$, respectively.

This work was supported by the U.S. Department of Energy, Office of Industrial Technologies, Advanced Industrial Concepts Division.

\section{References}

[1] Corliss, J. M., A. A. Putnam, M. J. Murphy, and D. W. Locklin, presented at the $105^{\text {th }}$ ASME Winter Annual Meeting, New Orleans, La., Paper No. 84-JPGC-APC-2, Dec. 1984.

[2] Bowman, C. T., "Control of CombustionGenerated Nitrogen Oxide Emission: Technology Driven by Regulation," Invited Lecture at Twenty-Fourth Symposium
(International) on Combustion The Combustion Institute, 1992/pp. 859-876.

[3] Keller, J. O., and I. Hongo, "Pulse Combustion: The Mechanisms of $\mathrm{NO}_{\mathrm{x}}$ Production," Combust. Flame, 80: 219-237, 1990.

[4] Keller, J. O., P. K. Barr, R. S. Gemmen, "Premixed Combustion in a Periodic Flow Field. Part I: Experimental Investigation" Combust. Flame, 99: 29-42 (1994).

[5] Barr, P. K., J. O. Keller, "Premixed Combustion in a Periodic Flow Field. Part II: The Importance of Flame Extinction by Fluid Dynamic Strain", Combust. Flame, 99: 43-52 (1994).

[6] Barr, P. K., J. O. Keller, T. T. Bramlette, C. K. Westbrook, and J. E. Dec, "Pulse Combustor Modeling: Demonstration of the Importance of Characteristic Times," Combust. Flame, 82: 252-269 (1990).

[7] Bramlette, T. T. and J. O. Keller, "Method and Apparatus for the Control of Fluid Dynamic Mixing in Pulse Combustors", U. S. Patent No. 5118281, June 2, 1992.

[8] Keller, J. O., L. L. Smith, and R. W. Dibble, "Phase Resolved PLIF Measurements of $\mathrm{CH}$ in a Strongly Oscillating Flow Field," Poster presentation at The Twenty Fourth Symposium (International) on Combustion, The Combustion Institute, Sydney Australia, July 1992.

[9] Lasheras, J. C., Linan, A. Lecuona, A., and Rodriquez P., "Vorticity Dynamics in ThreeDimensional Pulsating Co-Flowing Jet Diffusion Flames," Twenty-Fourth Symposium (International) on Combustion, The Combustion Institute, pp.325, 1992. 



\title{
Coal and Biomass Combustion
}

\author{
Introduction
}

A broad program of research is underway to gather fundamental information on important features of the combustion of pulverized coal and biomass fuels. An important element in this work is the development of improved sensors to monitor, in situ and in real time, process streams derived from the combustion of these fuels. Experiments are conducted at bench-, pilot-, and full-scale and are aimed at acquiring extensive qualitative and quantitative data, which are being used to develop predictive models that describe the combustion behavior of both the organic and inorganic constituents of commercially important coals and biomass fuels. The work is being done collaboratively with: combustion equipment manufacturers and electric utilities; the Pittsburgh Energy Technology Center; the National Renewable Energy Laboratory; and with colleagues from universities in the U.S. and abroad.

The experiments use well-controlled laboratory flow reactors to obtain timeresolved data during the combustion of wellcharacterized coals and raw biomass, as well as oils and chars derived from the pyrolysis of biomass. These reactors simulate the combustion environments in industrial boilers and furnaces. The reactors are instrumented with advanced optical diagnostics for in situ observation of the size, temperature, and velocity, or residence time, of reacting particles for narrowly size-classified coal samples. High-speed cinephotography is applied routinely to monitor the unique combustion behavior of biomass oils. The experiments are designed to measure the kinetic rates, chemical mechanisms, and products of combustion of both liquid and solid fuel feedstocks. These data are providing critical ingredients to new predictors - useful engineering models - of the combustion of these important fuels, based upon chemical and physical descriptors of the fuel and of the combustion environment.
Considerable resources are also being devoted to determining the combustion behavior of the inorganic species imbedded in the fuel matrix. Our pilot-scale facility, the Multifuel Combustor (MFC), simulates the combustion and deposition environments found in commercial boilers fired with coal or biomass fuels. The MFC is being used to conduct in situ measurements of the transformation and deposition of inorganic species on simulated boiler steam tubes during the combustion of a broad suite of pulverized coals, raw biomass fuels, and biomass pyrolysis oils. In this work we are developing and validating spectroscopic methods to detect, in situ, the chemical species in the deposits as a function of time.

In each of our experiments, in addition to the in situ optical measurements, extensive chemical analyses of isokinetically captured samples are conducted off line. Scanning electron microscopy and high resolution transmission electron microscopy are being applied to determine the morphology and composition of these captured samples. The latter technique, using char samples obtained as a function of combustion residence time in our laminar flow reactor, has been crucial to developing a fundamental understanding of the kinetics of char oxidation during the late stages of combustion (char burnout). For both coals and biomass fuels, data from these three reactors are being used to develop industrially oriented, engineering models to predict ash deposition rates, as well as to predict the character (e.g., chemical composition, emissivity, tenacity, and removability) of ash deposits formed under conditions representative of both conventional boilers and of advanced, 21st-century, coal and biomass utilization equipment.

\section{D.R. Hardesty}




\section{Heterogeneous Kinetics in the Late Stages of Coal Combustion}

The late stages of char combustion have a special technological significance, due to the economic incentives for achieving carbon conversions greater than 99\%. At the CRF, new optical and imaging techniques have been used to measure kinetics in the final stages of coal combustion and to investigate extinction and final burnout phenomena

\section{R. H. Hurt, K. A. Davis, and J. Ross'1}

The late stages of char combustion have a special technological significance, as carbon conversions of $99 \%$ or greater are typically required for the economic operation of commercial-scale boilers. Achieving such high conversions is difficult in certain cases, especially in connection with boilers retrofitted with combustion zone modifications for $\mathrm{NO}_{X}$ abatement.

At the CRF, two independent optical techniques have been used to make kinetic measurements in the important late stages of combustion. The first technique uses in situ optical measurements on entrained char particles burning in a laminar flow reactor, coupled with a recently proposed criterion [1] for distinguishing inorganic from carbon-rich particles by measurement of their spectral emissive factors in the near-infrared.

Based on these experiments, char particle combustion lifetimes can be conveniently depicted as trajectories on a plot of particle temperature vs. emissive factor, as shown in Fig. 1. Two distinct stages are revealed in the char combustion process: (1) a rapid, hightemperature combustion stage at low carbon conversion, followed by (2) a large decrease in particle temperature at roughly constant optical properties, initiating a final burnout stage that occurs slowly and at low temperatures, changing the optical properties of the particle to those characteristic of ash.

Similar behavior is observed in captiveparticle imaging experiments depicted in Fig. 2. In these experiments, solid fuel particles of 50 to $300 \mu \mathrm{m}$ in diameter are

\footnotetext{
$\overline{1_{\text {TAD Technical Services }}}$
}

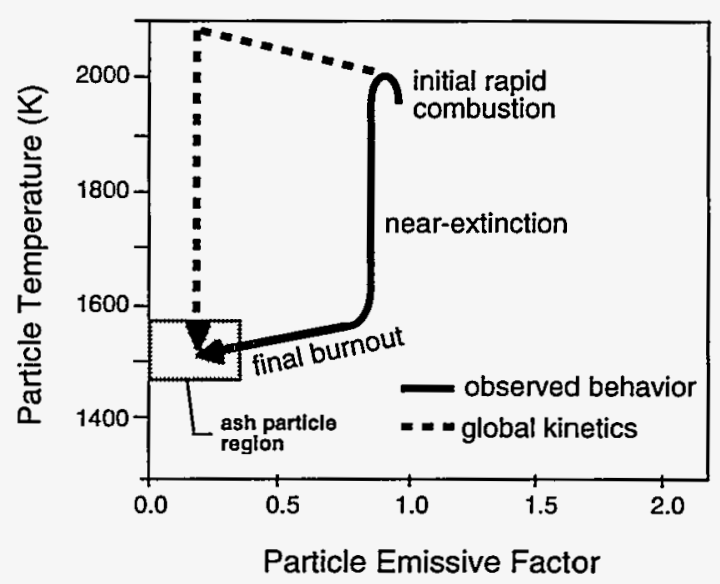

Figure 1. Example char particle combustion lifetime plotted as a trajectory in temperature/emissive-factor space.

combusted on a very low density particle support while their burnout behavior is imaged at high resolution using a long-focallength microscope system. The microscope has been modified for simultaneous imaging of reflected visible light and near infrared incandescence.

A typical dual-image sequence is shown in Fig. 3, along with time-resolved radiance temperatures determined from digitization of the near-infrared images. The combined data and images show a period of bright incandescence from 0.8 to 1.2 seconds, followed by a relatively abrupt drop in temperature of $125 \mathrm{~K}$ and a long, slow, nearlyisothermal, final burnout to a carbon-free ash particle. The gradual decrease in radiance temperature at long times is due, in part, to decreases in emissivity, but the initial rapid 


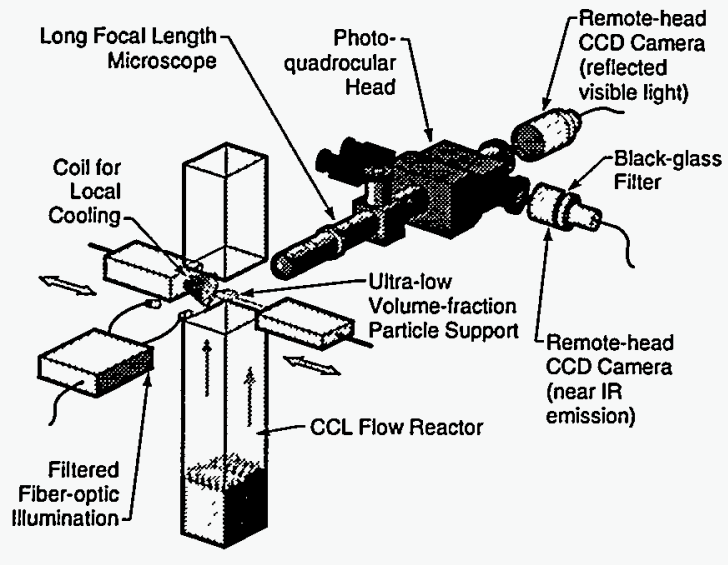

Figure 2. Schematic of the captive particle imaging apparatus.

decrease reflects a sudden decrease in particle temperature.

Kinetic analysis indicates that the abrupt temperature decrease is a "nearextinction event" caused by a loss of global reactivity rather than a true extinction event, which involves a mathematically discontinuous change in equilibrium particle temperature. For many particles, the loss of global reactivity can be related to changes in the carbonaceous phase occurring during combustion[2].
The asymptotic nature of the char combustion process has important implications for the modeling of carbon burnout in furnaces. Current global models typically used by U.S. industry assume a reactivity that is independent of conversion. Consequently, these models predict high particle temperatures and rapid heterogeneous combustion rates until the solid fuel is completely consumed (indicated by the dashed curve in Fig. 1). This simplified approach leads to a systematic error-carbon persists well beyond the point at which global kinetic models predict complete burnout.

This work was supported by the DOE, Office of Fossil Energy, Pittsburgh Energy Technology Center's ARETD Program.

\section{References}

[1] Hurt, R. H. "Reactivity Distributions and Extinction Phenomena in Coal Char Combustion Energy and Fuels 7:721, 1993.

[2] Davis, K.A., Hurt, R.H., Yang, N.Y.C., and Headley, T.J. "The evolution of char chemistry, crystallinity, and ultrafine structure during pulverized coal combustion" to appear in Comb. and Flame, 1994.

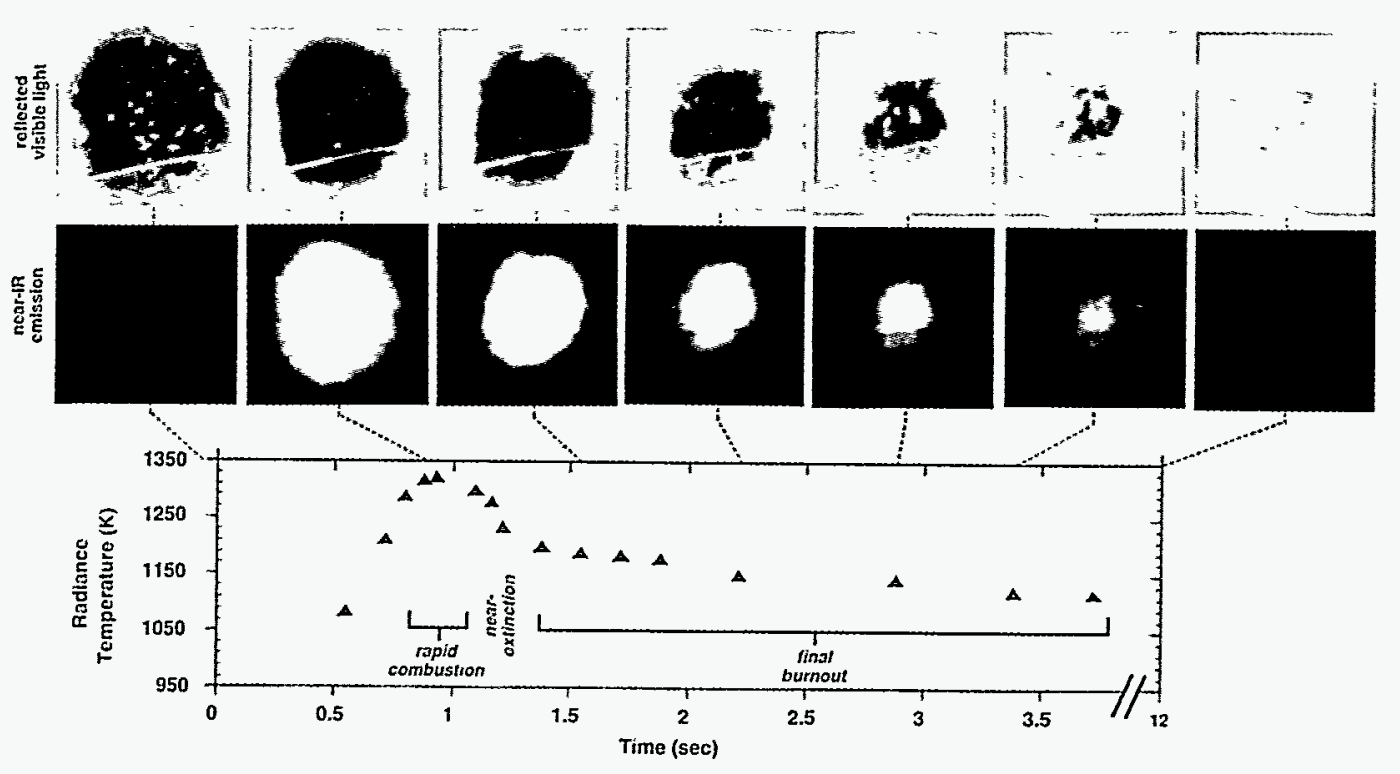

Figure 3. Dual-camera captive-particle image sequence for a typical Illinois \#6 coal-char particle of roughly $200 \mu \mathrm{m}$ in size burning in $6 \mathrm{~mole}-\%$ oxygen at a steady-state gas temperature of $1250 \mathrm{~K}$. 


\section{Materials Diagnostics Applied to Solid-Fuel Combustion Research}

Materials diagnostics are providing important new insights into solid fuel combustion

behavior. High-resolution TEM fringe-imaging is used to characterize crystalline

transformations of char carbon that are believed to be an important factor determining carbon

burnout levels in practical combustors.

\section{N. Y. C. Yang, ${ }^{1}$ T. J. Headley, ${ }^{2}$ R. H. Hurt, K. A. Davis, and M. J. Wornat}

In suspension-fired combustion systems, powdered solid fuels are injected into furnaces at high velocities, where they undergo rapid heating and pyrolysis followed by high intensity combustion of the residual char. At the subsecond time scales of this process, the crystalline structure of the char is not necessarily fully established prior to the onset of oxidation, but can continuously evolve. The crystalline or mesoscopic order in chars has long been known to have an important influence on their combustion reactivities, and thus high temperature structural ordering processes can have an important effect on reactivity in the late stages of burnout.

Very little is known about carbon crystalline transformations occurring at the high temperatures and short times characteristic of particle trajectories in entrained-flow combustors. CRF researchers have been collaborating with materials scientists at Sandia's sites in both California and New Mexico to study crystalline structure evolution using high-resolution transmission electron microscopy (HRTEM) fringe-imaging. Char samples are hand-ground to a fine powder, placed on a holey carbon grid, and examined at moderate magnification to find wedge-shaped fragments that are optically thin at the edge. A number of such edge regions are then photographed at high magnifications (approximately 2,000,000 x) in fringe-imaging mode, and representative fields of view selected and reproduced as seen in Figure 1. Individual graphitic layers with their planes perpendicular to the image plane

\footnotetext{
${ }^{1}$ Center for Materials and Applied Mechanics

2 Materials and Process Sciences Center
}

are clearly distinguishable as lines, separated by a distance that approximates, but is typically somewhat greater than, the characteristic interlayer spacing for graphite, $0.335 \mathrm{~nm}$.

Figure 1 presents fringe images of partially oxidized Pittsburgh \#8 coal char as a function of combustion residence time. The images reveal information on aromatic layer lengths, stacking depths, degree of layer curvature, and overall sample anisotropy (degree of orientational correlation among the layers).

$47 \mathrm{msec}$
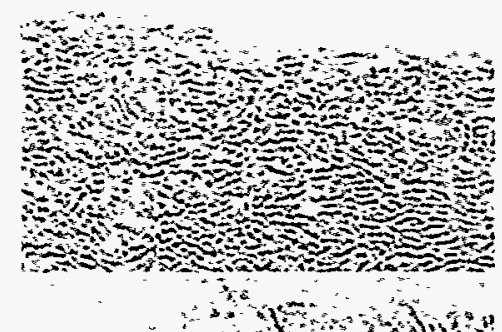

72 msec
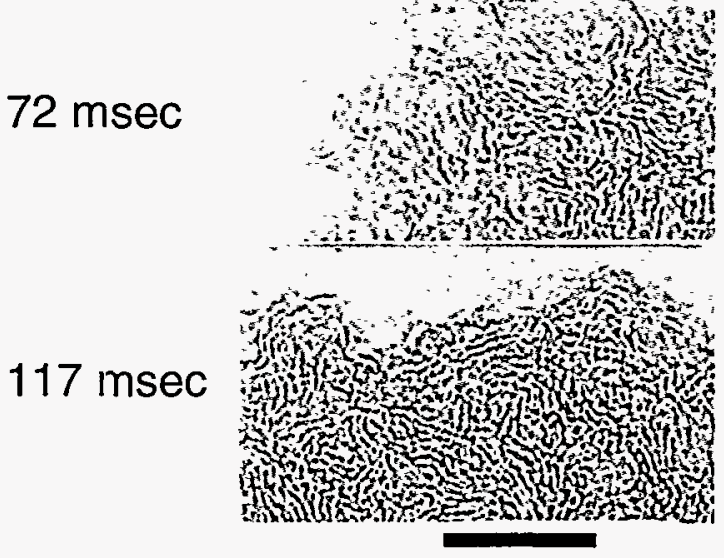

$10 \mathrm{~nm}$

Figure 1. HRTEM fringe-images of Pittsburgh \#8 coal chars sampled at three residence times in Sandia's high-temperature, entrained-flow reactor. 
For the particular example of Pittsburgh \#8 coal, combustion is accompanied by a distinct increase in the degree of order (see Fig. 2). In the general case, the properties of the parent organic material exert a strong influence on the degree of crystalline order and on the timing of the ordering process.

Based on our HRTEM analyses of four coal and two biomass chars sampled at different stages of oxidation, the rankdependent behavior can be described as follows. Upon rapid heating, low-rank, oxygen-rich precursors (biomass chars, lignites) behave as thermosetting materials and give rise to disordered cross-linked carbon structures that undergo limited rearrangement during subsequent combustion. Mid-rank bituminous coals (Illinois \#6, Pittsburgh \#8) pyrolyze to form disordered young chars with lower cross-link densities and a propensity to undergo significant rearrangements during the char combustion stages. Experiments on one highrank, low-volatile bituminous coal indicate significant ordering during pyrolysis, most likely by liquid crystal formation in the fluid phase, to yield highly anisotropic, ordered chars early in the combustion process. Continued thermal treatment perfects the initial crystalline order without requiring extensive lattice rearrangement.

An initial attempt has been made to extract quantitative information from the fringe images though digital image analysis [1]. A custom image analysis routine has been devised that utilizes Fourier transform filtering followed by object-oriented algorithms for sorting and sizing individual aromatic layers.

Applying this routine to partially reacted, Illinois \#6 coal chars yields crystalline structural parameters as shown in Fig 2. The mean crystallite diameter is relatively constant during combustion, while the fraction of turbostratic carbon - the material in a recognizably ordered phase - increases sharply. This increase in crystallinity correlates well with the decrease in the combustion reactivity measured over this time period by in situ optical techniques.

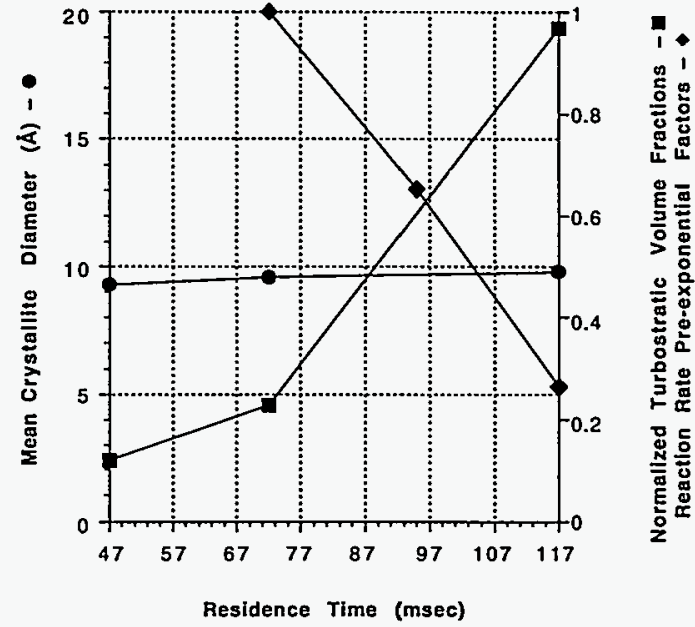

Figure 2. Crystalline structural parameters for Illinois \#6 chars estimated from fringeimages by digital image analysis.

Both laboratory experiments and the analysis of residual carbon samples from industrial boilers indicate that chars are progressively deactivated during high temperature combustion, and that this deactivation makes it difficult to burn out the remaining fraction of the parent fuel carbon. Mesoscopic nano-scale rearrangements in the crystal lattice are believed to be an important contributing mechanism to this reactivity loss for bituminous coals. This insight has important implications for the design and operation of commercial boiler systems.

This work was supported by the DOE, Office of Fossil Energy, through the Pittsburgh Energy Technology Center's Advanced Research and Technology Development Program.

\section{References}

[1] Davis, K.A., Hurt, R.H., Yang, N.Y.C., and Headley, T.J. "The evolution of char chemistry, crystallinity, and ultrafine structure during pulverized coal combustion" to appear in Comb. and Flame, 1994. 


\section{Combustion of Biomass-Derived Pyrolysis Oils}

Biomass-derived oils represent a renewable energy option for application in turbines, diesel engines and boilers. Sandia is investigating their combustion behavior and pollutant production as compared to traditional petroleum-derived oils. Single droplet and spray combustion studies allow us to identify the fundamental and practical issues associated with their use.

\section{K. A. Davis, M. J. Wornat, S. P. Huey, A. J. Salmi, B. G. Porter, J. Ross, ${ }^{1}$ G. C. Sclippa, ${ }^{1}$ B. M. Jenkins, ${ }^{2}$ F. Winter, ${ }^{3}$ and L. L. Baxter}

The increasing use of wood wastes, agricultural residues, and fuel-dedicated, fastgrowing crops offers the most promising nearterm option for displacing fossil fuels with an indigenous, renewable resource. Although many forms of biomass can be burned directly, pyrolysis and liquefaction can lead to a more valuable fuel for many applications. While the development of systems for the production of biomass-derived pyrolysis oil has been extensive, the combustion behavior of these oils is largely uncharacterized.

In an attempt to evaluate the suitability of these pyrolysis oils for use in practical energy conversion systems, two CRF experimental systems are being used to investigate their combustion characteristics - the Biomass Fuels Combustion System (BFCS), illustrated in Figure 1, and the Multifuel Combustor (MFC).

The BFCS comprises a laminar entrainedflow reactor, an aerodynamic droplet generator, a video imaging system, and a water-cooled sampling probe. The aerodynamic droplet generator produces a stable stream of uniformly-sized, well-spaced droplets from 80 to $600 \mu \mathrm{m}$ in diameter. The stoichiometry of the reactor's flame is tailored to produce $1600 \mathrm{~K}$ post-flame gases with oxygen concentrations of 14 to 33 mole $\%$. In-situ imaging of burning biomass droplets is accomplished with a super-VHS CCD camera, recorder, and monitor (back lighting is provided by a laser-triggered strobe).

\footnotetext{
${ }^{1}$ TAD Technical Services Corporation

${ }^{2}$ UC-Davis, Dept. of Biological \& Agricultural Eng.

${ }^{3}$ Technical Univ. of Vienna, Dept. of Chem. Eng.
}

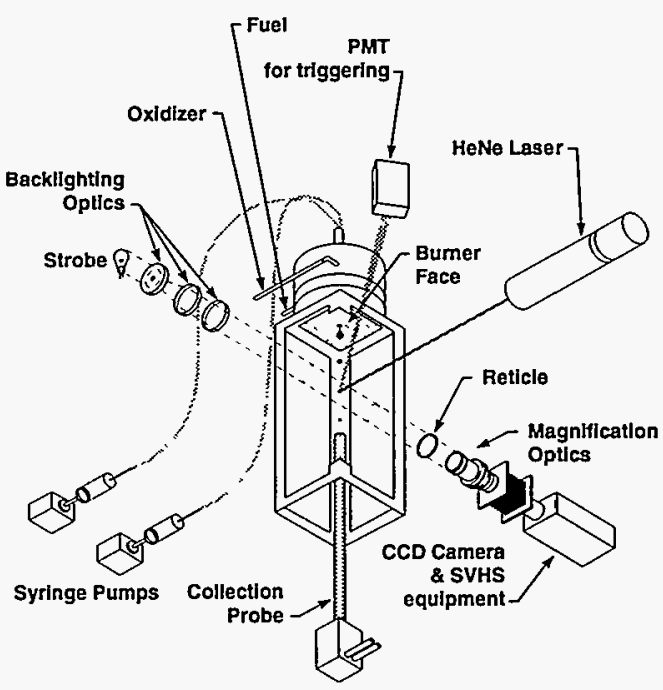

Figure 1. Biomass Fuels Combustion System for the investigation of single droplet combustion.

Sampling of partially reacted droplets is accomplished with the use of a temperaturecontrolled, limited-convection receptacle that collects the droplet stream while limiting evaporation.

The MFC is a pilot-scale facility designed to simulate commercial combustion furnaces, but is highly instrumented with diagnostics and controllers to provide detailed characterization of gas compositions and reactions, solid reactions, and surface interactions.

Biomass-derived pyrolysis oils burn very differently from petroleum-based fuel oils. Most of these differences can be traced to the inherently high oxygen content of biomass. Typical fuel oils exhibit evaporation coefficients of approximately $0.8 \mathrm{~mm}^{2} / \mathrm{g}$ and display little or no secondary atomization due 
to disruptive burning. Biomass-derived pyrolysis oils, on the other hand, exhibit evaporation coefficients of approximately 0.4 $\mathrm{mm}^{2} / \mathrm{g}$ and display variable, but significant, secondary atomization. The slower rates are directly and indirectly related to the high oxygen content of pyrolysis oils. Oxygenated fuels, in general, have lower heats of combustion and the increased water content of the pyrolysis oils acts as a heat sink during combustion (water is highly soluble in many of the oxygenated species present).

Secondary atomization can occur with petroleum-based fuels, but rarely leads to increased overall burning rates. The nature of the disruptive burning of the biomass-derived oils is very similar to that of typical water-infuel emulsions. As a droplet burns, volatile components in the droplet interior are superheated until their superheat limits are reached. The volatile components then undergo rapid gasification and the droplet is shattered. However, as illustrated in Figure 2, some biomass-derived oils, which are solutions rather than emulsions, undergo much more complete gasification.

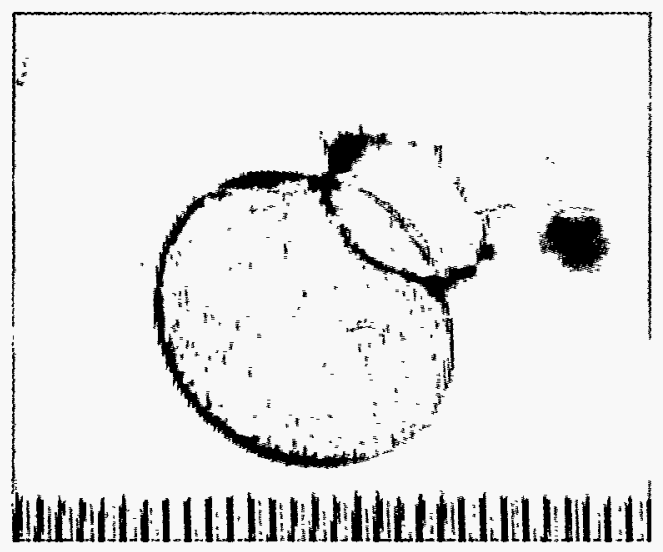

Figure 2. Microexplosion of a poplar-derived, pyrolysis-oil droplet $(100 \mu \mathrm{m} / \mathrm{div})$.

In addition to burning rates, another important feature of the combustion of these oils is particulate formation. Biomass-derived oils exhibit a strong tendency to form coke particulates during combustion. This behavior is, again, most likely the result of high oxygen levels, which result in polymerization at combustion temperatures. Analyses of unoxidized coke particulate indicate that oxygen-rich, nascent particles are formed and subsequently carbonized during the final stage of combustion.[1] It is important to note, however, that for specific oils such as the poplar oil of Fig. 2, which undergo nearly complete gasification during microexplosion, no coke particulate is formed.

In addition to this work in the BFCS, $\mathrm{NO}_{x}$ emissions have been examined in Sandia's MFC. The results of these experiments are useful for estimating the baseline $\mathrm{NO}_{x}$ levels that would be seen in a commercial boiler operating without any $\mathrm{NO}_{x}$ reduction technology. Peak baseline NOx emissions during the combustion of biomass-derived oils vary from 300 to $650 \mathrm{ppm}$, with lower levels associated with low nitrogen content fuels. Figure 3 shows the dependence of $\mathrm{NO}_{\mathrm{x}}$ levels on stoichiometry for a low-nitrogen-content oil.

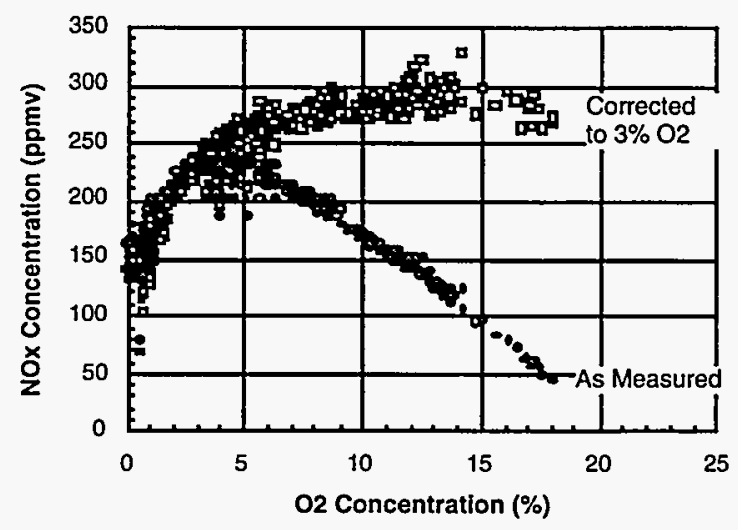

Figure 3. NOx levels as a function of effluent oxygen concentration for a low-nitrogencontent pyrolysis oil fired in Sandia's MFC.

Overall, the dependence of $\mathrm{NO}_{x}$ levels on stoichiometry and fuel nitrogen content agree qualitatively with those of other nitrogencontaining fuels. Measurements of thermal, prompt, and fuel $\mathrm{NO}_{\mathrm{x}}$ indicate that fuel $\mathrm{NO}_{\mathrm{x}}$ is the dominant formation mechanism $(>90 \%)$ and suggests that this $\mathrm{NO}_{x}$ is controllable with emission control technologies used with other nitrogen containing fuels.

This work was supported by the DOE, Biomass Power Program through the National Renewable Energy Laboratory, UC Davis and the Technical Univ. of Vienna.

\section{References}

[1] Wornat, M. J.; Porter, B. G.; Yang, N. Y. C. in press, Energy \& Fuels 1994. 


\section{Formation of Ash Deposits in Biomass-Fired Boilers}

Biomass-fired power generation is the leading combustion-based contributor of electric power from renewable sources both in the US and abroad. Firing of many fuels, in particular agricultural residues and energy crops, has led to formation of unmanageable ash deposits that curtail operations or substantially reduce boiler availability and efficiency. A series of laboratory and field tests have identified the mechanisms leading to deposit formation.

\section{L. Baxter, G. C. Sclippa, ${ }^{1}$ A. J. Salmi, B. M. Jenkins, ${ }^{2}$ T. R. Miles, ${ }^{3}$ T. R. Miles Jr., ${ }^{3}$ R. W. Bryers, ${ }^{4}$ and L. L. Oden ${ }^{5}$}

Biomass refers to a broad range of byproducts derived from lumber, agricultural, and municipal activities. Biomass-fired power stations combust biomass fuels to generate power from renewable, indigenous sources. Since the fuels are regrown each year, there is a net greenhouse gas emission of nearly zero. The power stations also remediate air quality and waste management problems by consuming material that might otherwise be burned in the field or disposed of in landfills. California is a leader in power generation from biomass, having over 70 biomass power stations, with a combined capacity of about $800 \mathrm{MW}_{\mathrm{e}}$.

Compared with more traditional forms of energy production (hydropower, coal, gas turbines, etc.) biomass power plants struggle to compete in overall energy conversion efficiency and cost of power. The industry in California currently enjoys price guarantees and other incentives that allow it to be profitable. However, these guarantees are beginning to expire and new regulations are compelling the industry to compete directly with more traditional forms of power production. A significant technical issue in increasing efficiency and profitability is the behavior of inorganic material during combustion, specifically deposition of ash on boiler surfaces.

In a collaboration including biomass-fired power stations, boiler manufacturers, small businesses, universities, and other government laboratories, ash deposition mechanisms are

\footnotetext{
${ }^{1}$ TAD Technical Services

${ }^{2}$ on sabbatical leave at the CRF from UC Davis

Department of Biological and Agricultural

Engineering

${ }^{3}$ T.R. Miles Consulting Design Engineers

${ }^{4}$ Foster Wheeler Development Corporation

${ }^{5}$ US Bureau of Mines, Albany Research Center
}

under investigation. Using a combination of laboratory tests in Sandia's Multifuel Combustor (MFC) and field tests in biomassfired boilers, the mechanisms leading to ash deposit formation were investigated. Figure 1 indicates the locations of biomass power generation facilities involved in this project.

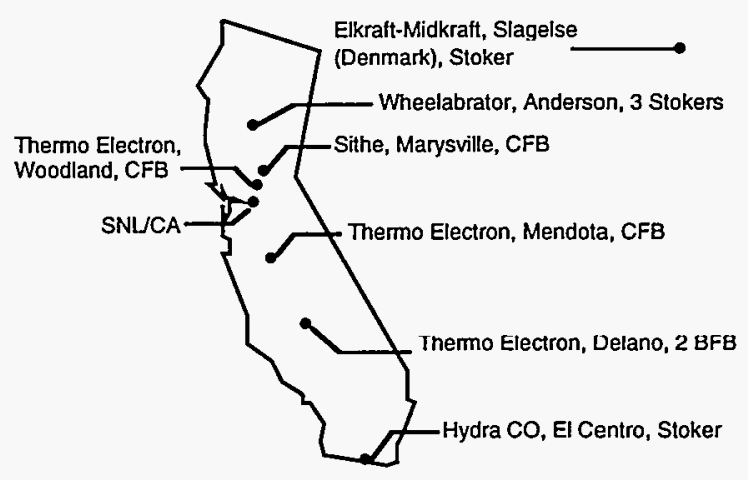

Figure 1.. Locations of commercial biomass power generation facilities involved in the field tests of biomass fuels.

Fuels used in these tests include woods, straws, nut shells, fruit pits, nut hulls, and non-recyclable paper. Boiler designs used in the field tests include stoker-fired moving grates, bubbling fluid beds, circulating fluid beds, auger-fired moving grates, and cigar burners. Two field tests were conducted in Denmark, with the remainder in California. This wide range of fuels and boiler designs provides an especially broad-based database.

The major mechanisms of deposition include impaction and adhesion of particles on surfaces, thermophoresis, condensation, and chemical reaction. Evidence of some of these mechanisms is indicated by the elemental 
composition of different regions of a wheat straw ash deposit generated during MFC tests (Fig. 2). The material most recently accumulated on the probe (the crown) is primarily composed of inertially impacted particles (e.g. $\mathrm{SiO}_{2}, \mathrm{P}_{2} \mathrm{O}_{5}$ ). Condensed vapors (e.g. $\mathrm{K}_{2} \mathrm{O}$ ) are most concentrated at the probe surface. Straws are composed of up to $20 \%$ inorganic material (excluding dirt or other adventitious sources), of which about $75 \%$ is silica and up to $15 \%$ is potassium. By contrast, potassium and other alkali metals often form vapors and condense on surfaces, as indicated by the trend in potassium concentrations.

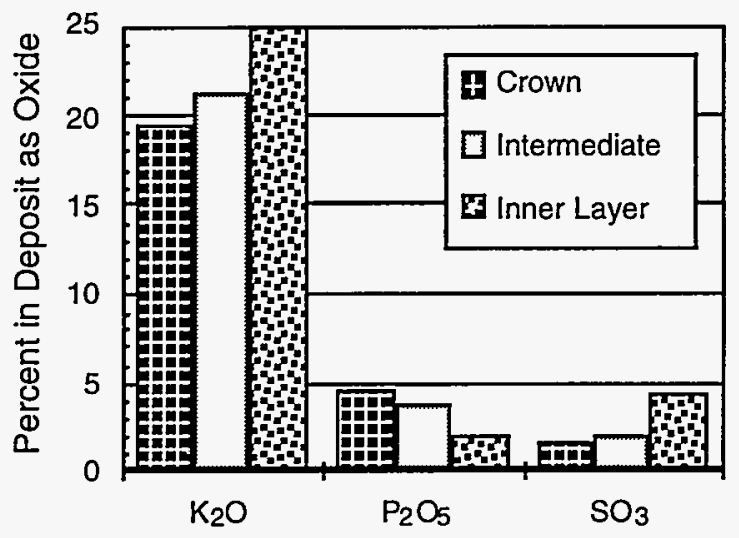

Figure 2. Elemental composition of three sections of wheat-straw deposit generated in the MFC. Silica concentrations are too high to conveniently illustrate here, but follow the same trend as phosphate.

Straws present particularly difficult ash deposition problems. Silica alone poses little problem, but when combined with materials such as alkali, silica can convert to problematic silicates as alkali condenses on its surface. Silicates commonly soften or melt at combustion temperatures. Molten material and sulfates lead to tightly bound surface deposits. Sulfates also have a high reflectivity, decreasing radiative heat transfer to the surface.

Similar samples were collected from commercial-scale power stations. Figures 3 and 4 illustrate one such station and the type of data gathered from it. This stoker-fired boiler was fed an $80 \% / 20 \%$ wood/wheat straw blend during the test burn. The fuel and deposit ash compositions differ significantly, illustrating selectivity in the deposition processes, consistent with the MFC tests.

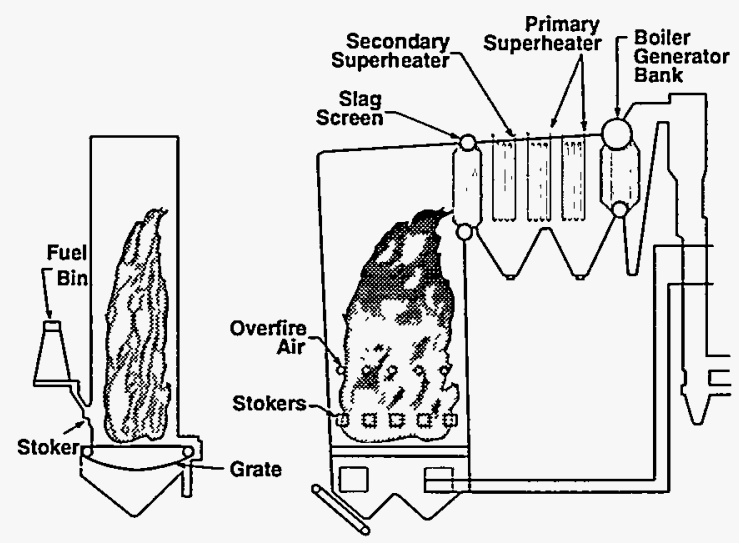

Figure 3. Schematic diagram of one of the US commercial-scale combustors used in the field tests of biomass ash deposition tendencies.

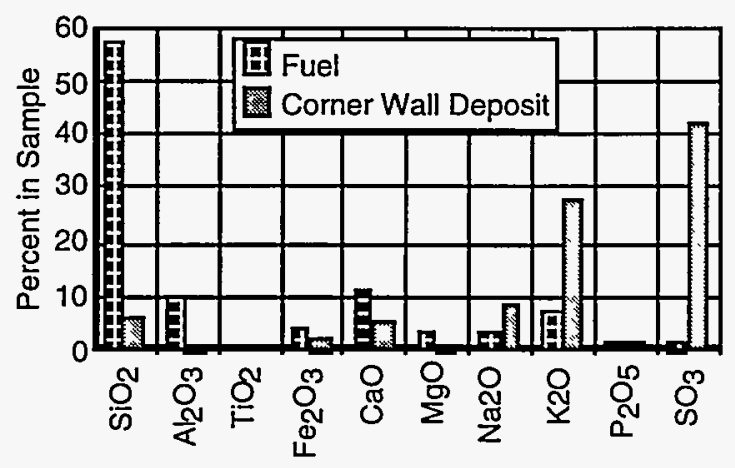

Figure 4. Composition of an ash deposit collected during a test burn of a blend of straw and wood in a commercial biomass-fired combustor.

This mechanistic understanding of ash deposition identifies means of selecting fuel blends and boiler operating parameters to manage the ash deposition problem. In future work, this understanding will be formalized in a computer code and will be applied by the biomass industry to more skillfully manage their combustors, decrease their costs, and increase their efficiency and reliability.

This work was supported by the Biomass Power Program of the U.S. DOE Office of Energy Efficiency and Renewable Energy in cooperation with the National Renewable Energy Laboratory , a consortium of biomass power industries, and the U.S. Bureau of Mines. 


\section{FTIR Emission Spectroscopy Applied to In Situ, Real-Time, Surface Chemistry Measurements}

Hostile environments common to many combustion systems prevent detailed analysis of surface reactions. Sandia recently developed a spectroscopic technique for monitoring chemical changes on surfaces in combustion environments. It has been instrumental in resolving several practical issues related to coal combustion.

\section{L. Baxter, G. C. Sclippa, ${ }^{1}$ A. J. Salmi, G. H. Richards, ${ }^{2}$ D. K. Ottesen, and J. N. Harb ${ }^{3}$}

The behavior of inorganic material during combustion of most solid fuels has a large impact on design and operation of commercial combustors due to formation of ash deposits. The CRF has developed a diagnostic method using Fourier Transform Infrared (FTIR) emission spectroscopy for in situ surface monitoring of such deposits under conditions similar to those found in full-scale boilers. This diagnostic has application to ash deposit formation, corrosion, and heat transfer analyses in coal-, biomass-, and waste-fired boilers. It is currently used in conjunction with the CRF's Multifuel Combustor (MFC) to analyze coal ash deposit properties.

The spectral features of the emissivity monitored by the spectrometer (Fig. 1) indicate chemical species present on the surface. There are four principal complexities: distinguishing radiation emitted from the relatively cool $\left(300-800{ }^{\circ} \mathrm{C}\right)$ surface from radiation reflected from the hotter $\left(900-1700^{\circ} \mathrm{C}\right)$ gases and soot-laden flame; resolving interferences from infrared-active gases; dealing with signals that are affected by the turbulent, particleladen, reacting flow; and interpreting spectral data from inhomogeneous, particulate, porous deposits with large temperature gradients. Sufficient progress in addressing these issues has been made to produce practical results and validate the quantitative emissivity data and semi-quantitative chemical species data [1-2].

\footnotetext{
${ }^{1}$ TAD Technical Services

${ }^{2}$ Brigham Young University graduate student working at the CRF

${ }^{3}$ Brigham Young University, Department of Chemical Engineering
}

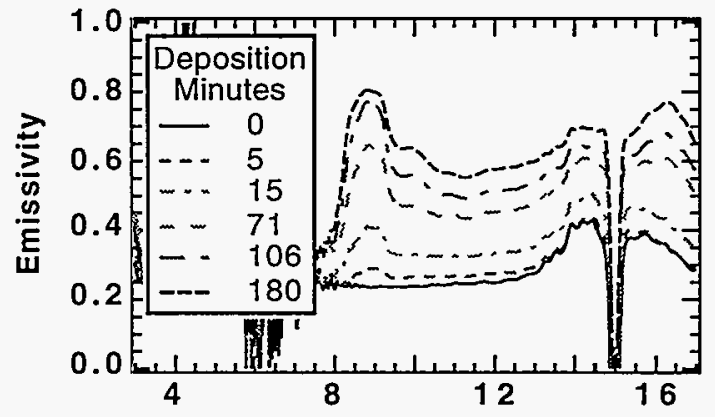

Wavelength, $\mu \mathrm{m}$

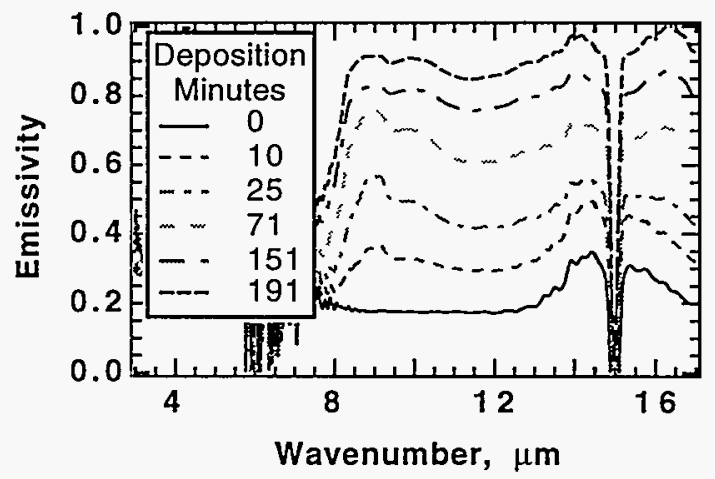

Figure 1. Time-resolved spectral emissivities collected in situ in the MFC for two similar low-sulfur coals. Emission from sulfates (near $8.8 \mu \mathrm{m}$ ) and silica (near $9 \mu \mathrm{m}$ ) differ from many of the silicates (10 to $13.5 \mu \mathrm{m}$ ) by a large ratio for one coal, but by a much smaller ratio for the second. Residual interference from gas-phase carbon dioxide (4.4 and $15 \mu \mathrm{m})$ and water $(5.8-7 \mu \mathrm{m})$ can also be seen.

The use of the diagnostic is illustrated by an application to utility boilers. Two western US coals of similar organic and inorganic elemental compositions and geographic origins were tested in utility-scale boilers. By all traditional analyses, these coals should behave 
similarly. Field experience indicated one coal produced more problematic ash deposits than the other. In the CRF experiments, the diagnostic was used to determine the reasons for the different behaviors.

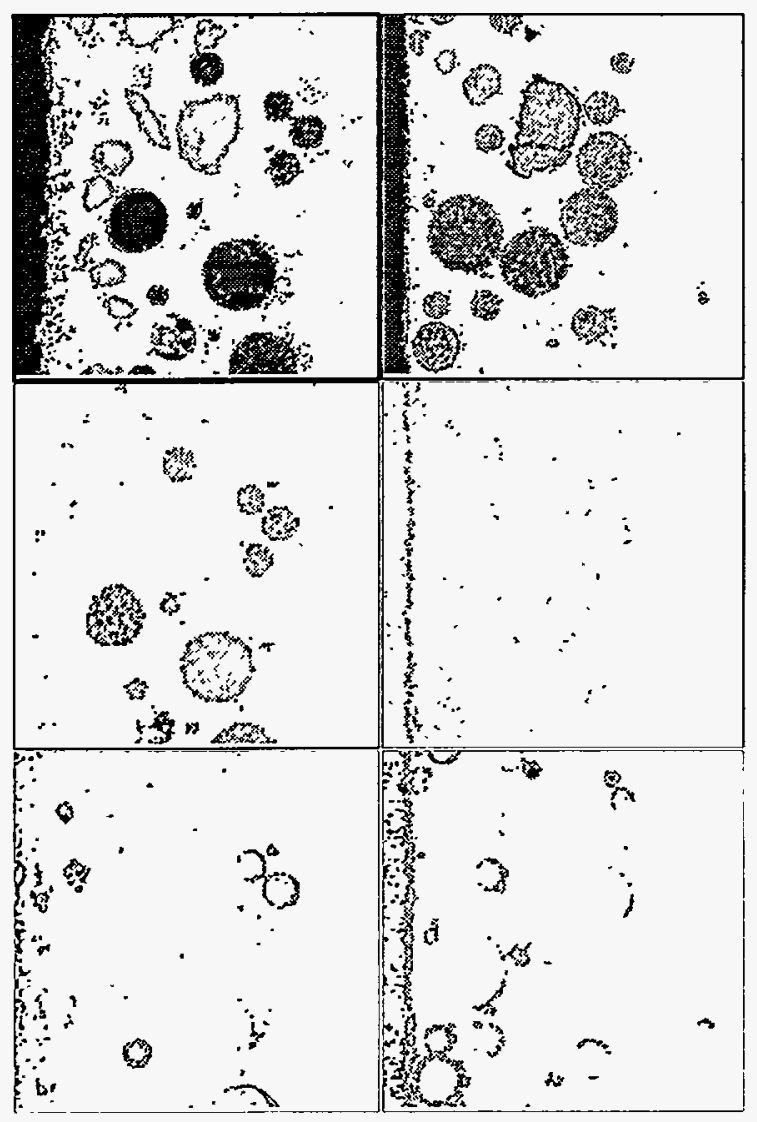

Figure 2. SEM images of cross-sections of deposits formed on a probe in the MFC. The probe surface is on the left of each image. The left column includes data from one coal, illustrating overall deposit structure (top), calcium map (center), and sulfur map (bottom). The right column illustrates similar data from the other coal, with the center image representing sodium rather than calcium. Each image represents approximately $125 \mu \mathrm{m}$ along each side.

The coals were tested in the MFC under conditions simulating different regions of a utility boiler. The species composition of ash deposits accumulating on temperature-regulated probes was monitored using the new diagnostic. Figure 1 illustrates the data in terms of spectral features of emissivities. While both deposits are composed of mixtures of silica, various silicates, and several sulfates, the rela- tive intensities of the emission features associated with these species differ in time and between samples. The deposit from one coal exhibits greater sulfate character whereas the deposit from the other coal has greater silicate character. Spectra from both samples indicate early deposits dominated by sulfate, with silicate character developing later.

Ex situ data of several types complement these in situ data. One example is cross sections of the same deposits, examined with scanning electron microscopy (SEM) (Fig. 2). The cross sections illustrate the initial layers of the deposits. A particle map and various elemental composition maps of the deposits confirm the chemistry indicated by the spectra in Fig. 1. For example, sulfate layers are seen to form on the surfaces of calcium oxide particles, but not on the surfaces of calcium silicate particles. An initial sodium sulfate layer is seen on the surface of one of the probes. As the deposit grows, the sodium sulfate disappears, whereas calcium sulfate peaks become more pronounced, consistent with a detailed analysis of the spectra.

Future applications of the technique include: investigations of high-temperature corrosion of metal surfaces in biomass and coal combustors; corrosion of silicon carbide in high-temperature, oxidizing, alkali-laden combustion gases; and determination of the effects of low- $\mathrm{NO}_{\mathbf{x}}$ burners on deposit chemistry. Work also continues in the areas of ash deposit analyses and analytical interpretation of composition data based on emissivities.

This work was supported by the U.S. DOE through the Pittsburgh Energy Technology Center's Direct Utilization Advanced Research and Technology Development Program and Brigham Young University's Advanced Center for Engineering Research on Combustion.

\section{References}

[1] Baxter, L.L., (1993) "In Situ, Real-Time Emission FTIR Spectroscopy as a Diagnostic for Ash Deposition During Coal Combustion," Proceedings of the Engineering Foundation Conference on The Impact of Ash Deposition on Coal-Fired Plants, Taylor \& Francis, New York,

[2] Baxter, L.L., G.H. Richards, D.K. Ottesen, and J.N. Harb, (1993) Energy \& Fuels, 7:755760. 



\section{Industrial Processing}

\section{Introduction}

We are engaged in a variety of projects in the area of industrial processing whose common theme is an emphasis on understanding the behavior of materials undergoing hightemperature conversion processes as a function of the chemical and physical properties of the starting material, as well as the key parameters that characterize the processing environment. These activities include fundamental theoretical and experimental works, as well as applied modeling and demonstration experiments. Included are:: research on the high temperature, combustion synthesis of new materials, such as silicon carbide and carbon-carbon composites; development of chemically reacting flow models to predict plasma-based semiconductor manufacturing processes; research on the destruction of hazardous wastes using supercritical water oxidation (SCWO); and development of new optical sensors for monitoring, in situ, steelmaking processes.

The experiments employ laboratory reactors that simulate the key features of practical industrial processing systems and permit the temporal resolution required to interrogate the details of these complex, multiphase, high-temperature processes.. We make use of the wide array of laser diagnostics available in the CRF to measure temperatures, velocities, and species compositions. Rapid-quench sampling systems are also used, in combination with mass spectrometric analysis of extracted materials, to obtain detailed information on both reaction intermediates and products. In this work, we collaborate with partners from both the U.S. industrial community and universities.

In our research on high temperature synthesis of silicon carbide, we are developing an understanding of both gas phase and surface reactions, within the silicon-carbonhydrogen-chlorine system. Of interest are chemical systems that yield solid silicon carbide in the form of surface coatings, powders, and high-density composites. In this work, we are using a new capability at the CRF, the High-Temperature Flow Reactor. Models are used to describe the deposition of silicon carbide and the behavior of industrial reactors that rely on particle-enhancement to augment the rates of silicon carbide deposition. Quantum chemistry is used to develop the thermochemical parameters required in both the models and the experiments. For carboncarbon composite manufacturing, we are exploring the non equilibrium, chemically reacting flow processes that produce a matrix of chemically reactive intermediates during the pyrolysis of pure hydrocarbon precursors such as cyclohexane.

Our research on the SCWO destruction of toxic aqueous wastes uses two unique experimental reactors to control and monitor key operational features of this promising technology. Using laser diagnostics, one reactor yields time-resolved data under constant volume reaction conditions; a second flow reactor allows measurement of reactants, intermediates and products at constant pressure. For a broad range of aqueous materials, including both surrogates and wastes such as munitions, we have measured destruction efficiencies, chemical reaction kinetics, and oxidation mechanisms.

In addition, we are working directly with major U.S. industrial consortia. Under a CRADA with SEMATECH, we are applying Sandia-developed chemically reacting flow codes to help design and optimize semiconductor processing equipment. Through the DOE Metals Initiative, we are working with the American Iron and Steel Institute to develop advanced prototype sensors for use by the U.S. steelmaking industry.

\section{D.R. Hardesty}




\title{
Understanding the Gas-Phase Decomposition of Ceramic Precursors
}

\author{
Process models verified by experimental measurements are needed to lower the cost and \\ speed development of ceramic materials for energy applications
}

\section{D. Allendorf, T. H. Osterheld, S. M. Ferko, and C. F. Melius}

The manufacture of refractory ceramics is a major U.S. industry, producing everything from coffee cups to packaging for integrated circuits. In applications where hightemperatures, corrosion, and wear limit the kinds of materials that can be used, ceramic coatings and composites can withstand temperatures and environments more severe than most metals and can do so under load. This makes them candidates for many "hightech" applications, such as gas-turbines, steam reformers, heat exchangers, and combustion engine parts. Although "low-tech" consumer applications are plentiful, ceramics have, in many cases, failed to achieve their highest potential as replacements for metals and superalloys because their high cost limits their penetration into large-volume markets.

Silicon carbide $(\mathrm{SiC})$ is a ceramic of interest for a wide range of energy-saving industrial and transportation applications because of its stability at high temperatures, resistance to oxidation and corrosion, and high thermal conductivity. Batch processing and low deposition rates contribute to high costs for SiC materials. Processes under development offer the potential for lower-cost materials, but are difficult to optimize and scale up because of the complexity of the interactions between deposition chemistry and heat/mass transport. Computational models can provide valuable insight into the relationship between process variables and deposition rates, but require a detailed knowledge of the reaction chemistry to be quantitative. In the case of $\mathrm{SiC}$, gas-phase decomposition of methyltrichlorosilane $\left(\mathrm{CH}_{3} \mathrm{SiCl}_{3} ; \mathrm{MTS}\right)$, the typical precursor, is known to occur under deposition conditions. Unfortunately, the chemistry of chlorinated organosilanes is poorly characterized, making it difficult to predict MTS decomposition rates and the subsequent formation of products.

To better understand the gas-phase reactions of MTS, we applied experimental and computational techniques that have proven useful for the study of similar problems in combustion chemistry. Measurements of MTS decomposition were performed in a high-temperature flow reactor (HTFR), capable of reaching temperatures as high as $1500 \mathrm{~K}$ (Fig. 1). The reactor design allows MTS to be rapidly mixed at low pressures ( 25 torr) with a preheated carrier gas such as helium. The gases then travel through a constanttemperature zone, with a residence time determined by the location of a movable, water-cooled injector. Stable decomposition products and residual MTS are detected by mass spectrometric sampling through a quartz probe inserted into the gas flow.

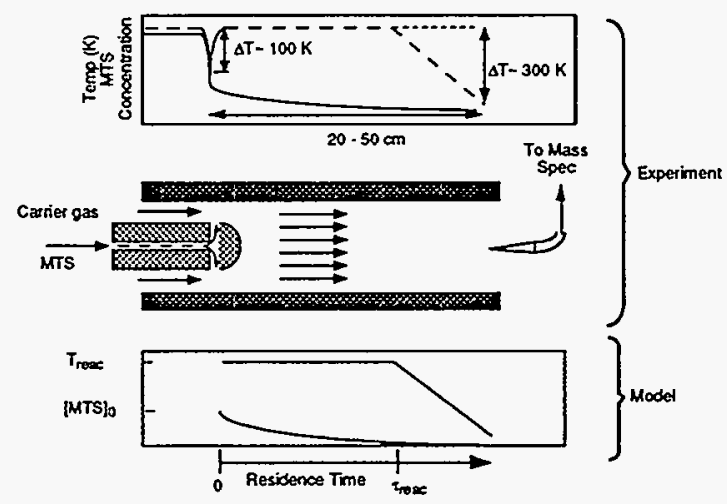

Figure 1. Schematic of the HTFR experiment and the plug-flow model used to simulate it.

The experiments show that MTS partially decomposes in helium under the conditions of the experiments (25 torr, 1183-1273 K), producing $\mathrm{SiCl}_{4}, \mathrm{CH}_{4}$, and $\mathrm{HCl}$. Measurements of the MTS decomposition rate are shown in Figure 2. The experimentally determined rate 
is in good agreement with the rate we predict using RRKM theory. This shows that the ratelimiting step under these conditions is $\mathrm{Si}-\mathrm{C}$ bond scission $\left(\mathrm{CH}_{3} \mathrm{SiCl}_{3} \rightarrow \mathrm{CH}_{3}+\mathrm{SiCl}_{3}\right)$. The fact that the measured rate is a factor of two faster than predicted could be due simply to uncertainty in the calculation. However, as we describe below, secondary reactions involving the initial products $\mathrm{CH}_{3}$ and $\mathrm{SiCl}_{3}$ can lead to a rate somewhat higher than the unimolecular rate predicted by RRKM.

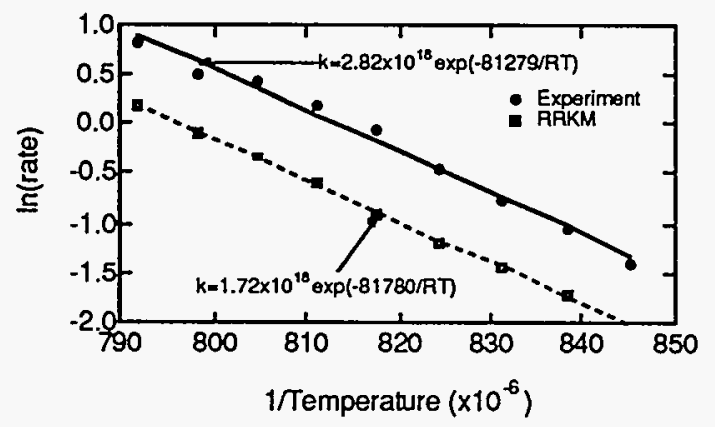

Figure 2. Comparison of MTS decomposition rates measured in the HTFR with rates predicted by RRKM theory.

Since the initial Si-C bond-breaking step does not lead to any of the observed products, additional reactions must be employed to fully explain the results. It is thus necessary to develop a multi-reaction mechanism to model the experimental data. The mechanism that we have developed is shown schematically in
Figure 3. Rate constants for some of these reactions are available from literature sources. However, since most of the reactions involving silicon-containing species are uncharacterized, these rates were estimated from analogous reactions or by theoretical methods. In a few cases, rates were fit to HTFR data, using a plug-flow model (Fig. 1) to predict the measured species concentrations.

Model predictions are in reasonable agreement with the relatively narrow range of the experimental measurements of MTS decomposition made to date. While recognizing this limitation, the model provides useful new insight into the MTS reaction chemistry. For example, slow steps in the mechanism can be identified and major product formation pathways (bold arrows in Fig. 3) can be identified. The model is thus a useful first step toward achieving a detailed understanding of the gas-phase chemistry of this $\mathrm{SiC}$ precursor. Since many of the rates used in the mechanism are uncertain, particularly those determined by fits to HTFR data, we are working to more firmly establish these rates by performing additional HTFR experiments over a wider range of temperatures and pressures and hy expanding our theoretical treatments of the various reactions.

This work was supported by the DOE, , Office of Industrial Technologies, Advanced Industrial Materials Program.

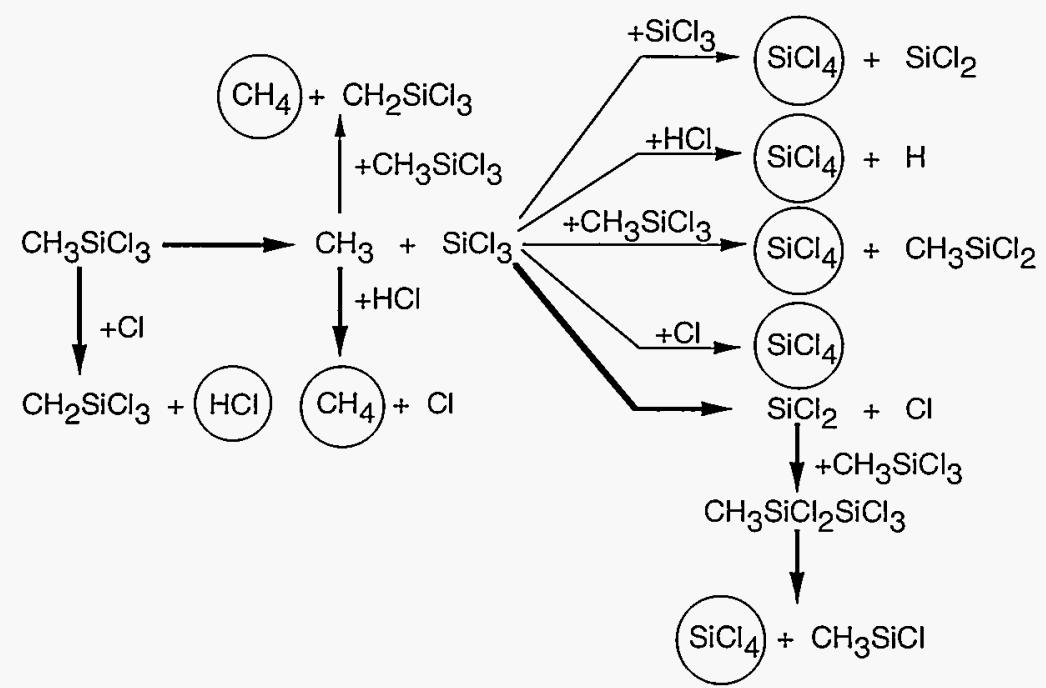

Figure 3. Reaction mechanism for the decomposition of MTS. Bold arrows indicate major reaction pathways. 


\section{Gas-Phase Chemistry Involved in the Deposition of Carbon from Cyclohexane}

The kinetics and product branching of cyclohexane pyrolysis have been measured in Sandia's High-Temperature-Flow-Reactor at very high levels of decomposition leading to formation of solid carbon. A simple model is provided that explains the experimental observations.

\section{T. H. Osterheld, M. D. Allendorf, and S. M. Ferko}

In the desire to develop stronger, harder, lighter, and higher-temperature-resistant products, industry is turning to composite materials. Carbon-carbon composites are among the most successful of these materials with applications ranging from brakes and clutches in aircraft and automobiles to rocket and jet engine components. Carbon-carbon composites have many advantages: a very low coefficient of thermal expansion, increasing strength with temperature to over $2500 \mathrm{~K}$, a sublimation temperature of over $3500 \mathrm{~K}$, high strength-to-density ratios and modulusto-density ratios, good thermal conductivity, and the ability to conduct electricity.

Unfortunately, as with many composite materials, high production costs limit exploitation of their unique properties. A recently developed process for the rapid densification of carbon could reduce production costs. In this process, the gas-phase precursor cyclohexane $\left(\mathrm{C}_{6} \mathrm{H}_{12}\right)$ is used to densify preforms of carbon fibers woven into a desired shape. Improvement and control of this process requires a better knowledge of the chemical transformation of cyclohexane to solid carbon. In fact, we are not aware of any work characterizing the gas-phase kinetics during this transformation. For these non-equilibrium processes a knowledge of the depositing species and the kinetics of their formation is crucial to attaining an understanding of the deposition process.

We examined the decomposition of cyclohexane in Sandia's High-Temperature-FlowReactor (HTFR) using mass spectrometric detection. See accompanying article in this section. This reactor allows us to identify reaction products and measure reaction rates. Cyclohexane decomposition levels were varied from low to complete conversion by varying residence time in the reactor's hot zone. We also conducted experiments at much longer residence times that provided information concerning the decomposition of cyclohexane reaction products. Initial cyclohexane concentrations varied from 1-8\% in a helium carrier gas.

We observed 28 different reaction products. In contrast to data obtained at short residence times by earlier studies [1], we observed significant amounts of polyaromatic hydrocarbons (PAH). Figure 1 shows the branching between cyclohexane and the major products as a function of residence time at $1163 \mathrm{~K}$. Concentrations are relative to the total signal at a given residence time and not to the initial cyclohexane signal. As shown in

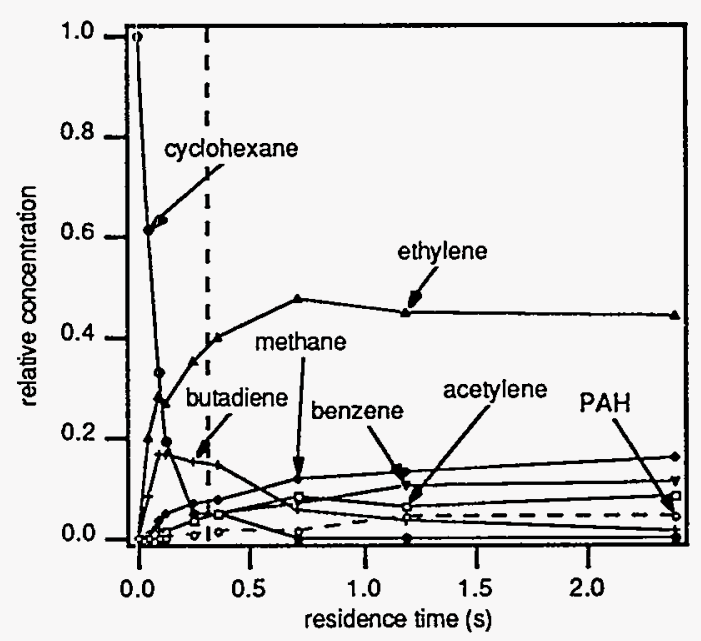

Figure 1. Dependence of measured concentrations of the principal reaction products on residence time at $1163 \mathrm{~K}$. Data points to the left of the vertical dashed line were obtained at 25 torr total pressure, while those to the right were obtained at 250 torr. 
Figure 1, the $\mathrm{PAH}$ reach a concentration of about $4 \%$, which corresponds to $16 \%$ of the total gas phase carbon.

To observe higher decomposition levels, we increased the reaction temperature to $1363 \mathrm{~K}$. The result of these experiments is shown in Figure 2. Note that cyclohexane decomposes too fast under these conditions to be observed. As shown in Figure 2, the major species at long residence time are hydrogen, methane, acetylene, ethylene, and benzene, which account for $99 \%$ of the gas-phase compounds. The primary reaction channel at this temperature appears to be the conversion of ethylene to acetylene. In contrast to the initial decomposition of $\mathrm{C}_{6} \mathrm{H}_{12}$, species concentrations approach relatively constant values at the longer residence times. However, simulation of the experiments shows that concentrations are still far from their equilibrium values. The slower reactivity arises from a decrease in the rates of radical chain reactions as reactive radical precursors are depleted.

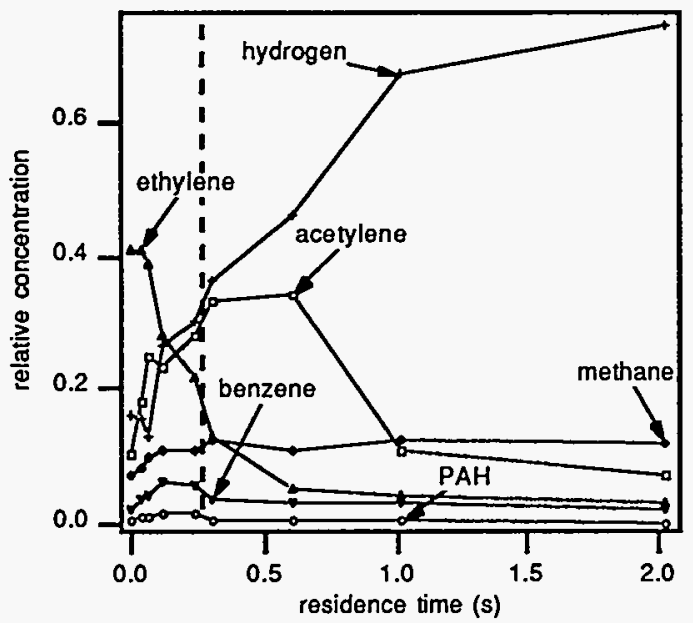

Figure 2. Dependence of measured concentrations of the principal reaction products on residence time at $1363 \mathrm{~K}$. Data points to the left of the vertical dashed line were obtained at 25 torr total pressure, while those to the right were obtained at $\mathbf{2 5 0}$ torr.

By accounting for the number of carbon atoms in each molecule, we can also measure the decrease in total gas-phase carbon with residence time. This presumably arises from losses at the reactor walls (i.e. deposition). Two kinetic regimes for carbon loss are observed. Disappearance of the first $30 \%$ is very rapid while the remaining carbon loss is much slower. Presumably, the first regime involves rapid deposition from radical species while the second regime involves slower deposition by direct adsorption of PAH, benzene, ethylene, acetylene, and methane. Consistent with this description, we observed a much smaller loss of methane at long residence time than of benzene or acetylene. This is consistent with the much lower sticking coefficient expected for methane.

Figure 3 provides a simple model for the global deposition of carbon that is consistent with our experiments. The initial stage of $\mathrm{C}_{6} \mathrm{H}_{12}$ decomposition provides a reactive pool of radicals and hydrogenated hydrocarbons. This reactive pool branches between direct deposition of radicals, condensation reactions to form $\mathrm{PAH}$, and fragmentation to generate lower molecular weight compounds. At long residence times, the gas-phase composition is relatively constant because radicals are no longer available to facilitate decomposition. At this point, deposition occurs by direct adsorption of stable molecules.

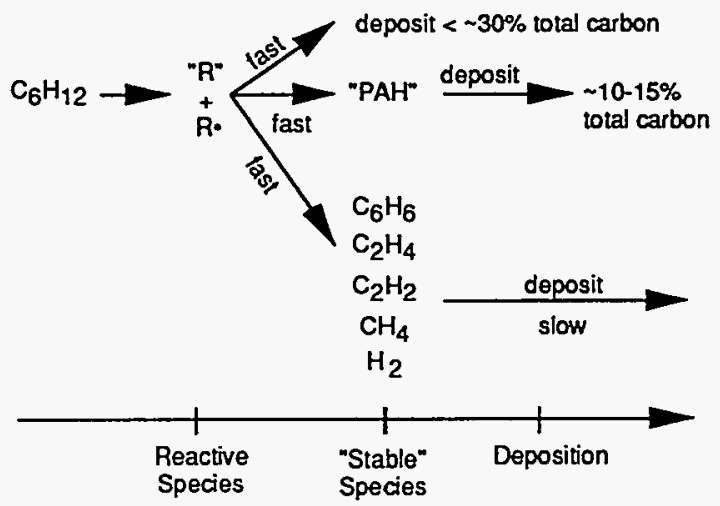

Figure 3. Simple decomposition model for cyclohexane.

In future work we plan to deposit cyclohexane on test samples to observe the dependence of microstructure and deposition rate on the gas-phase composition.

This work was supported by the DoD Advanced Research Projects Agency.

\section{References}

[1] F. Billaud, M. Duret, K. Elyahyaoui, and F. Baronnet, "Survey of Recent Cyclohexane Pyrolysis Literature and Stoichiometric Analysis of Cyclohexane Decomposition," Ind. Eng. Chem. Res. 30, 1469-1478 (1991) 


\title{
Modeling of Plasma-Based Semiconductor Manufacturing Equipment
}

\author{
Successful fabrication of integrated circuits from silicon wafers involves hundreds of \\ deposition and etching steps, all of which must be done with a high degree of uniformity \\ across the wafer surface. Chemically-reacting flow models are being used to aid the design \\ and operation of equipment for silicon wafer etching and deposition.
}

\section{S. R. Vosen, E. Meeks, J. W. Shon, R. S. Larson and R. J. Kee}

The development of more complex integrated circuits with smaller features is resulting in etch and deposition equipment based on lowpressure (a few Torr) plasma-based processing. In addition, environmental regulations are providing a push towards replacing aqueous processes with plasma-based (dry etch) processing, where possible.

The next generation of electronics requires features as small as 0.25 micrometers on wafers 200 to 300 millimeters in diameter, resulting in process specifications that will be difficult to achieve. The modeling work described here is part of a larger plasma-modeling effort being conducted under a CRADA with SEMATECH to develop and apply computer models to aid in the design and optimization of electronics processing equipment.

We are working with SEMATECH and two American equipment manufacturers to develop an etching process called Chemical Downstream Etch (CDE) (Fig. 1). $C D E$ is being developed for poly-buffered LOCOS stripping, which requires an isotropic etch with no charged etchants at low temperature.

Typical CDE systems consist of a microwave plasma source to produce etchants from feed-stock gases, a transport tube where the charge is neutralized, and a process chamber, where wafer etching occurs.

Chemically reacting flow models of the $\mathrm{CDE}$ process, including compilation of neutral and electron-driven kinetics, have been developed and are being used to predict performance as a function of operating parameters.

A neutral and plasma reaction mechanism is necessary for the microwave plasma-source model to predict the variation of composition as a function of flow rates, pressure, and power input. The gases of interest include mixtures of nitrogen trifluoride $\left(\mathrm{NF}_{3}\right)$, carbon tetrafluoride $\left(\mathrm{CF}_{4}\right)$, and oxygen $\left(\mathrm{O}_{2}\right)$. Our mechanism consists of 18 species and 100 reactions. The compilation of an accurate and sufficiently detailed mechanism ensures consistent chemical modeling from the plasma source to the wafer surface. The plasma source model that we have developed shows that under CDE conditions the plasma produces a large atomic-fluorine concentration (about 90 mole percent) and the gas is highly electronegative, with dominant charged species being $\mathrm{F}^{-}$and $\mathrm{NF}_{2}{ }^{+}$.

Figure 1. Schematic of a chemical downstream etcher. 
A plasma plug-flow model predicts the ion and radical species concentrations in the channel that connects the plasma source and the process chamber. This model includes the mass and energy transport of charged and neutral species through the transport tube, as well as gas-phase and surface reactions. Surface reactions are very important in this region, with electrons recombining with positive ions rapidly. Because the gases are electronegative, the dominant charged species $\left(\mathrm{F}^{-}\right.$ and $\mathrm{NF}_{2}{ }^{+}$) persist many tens of centimeters down the tube.

The process chamber model consists of a showerhead model and one- and two-dimensional reacting-flow models. The showerhead model is used to improve flow uniformity at the transition between the transport tube and the etch chamber. Application of this model has successfully improved the design of new equipment. The one- and two-dimensional flow models include the effects of gas-phase reactions, surface recombination, and silicon etching by atomic fluorine. The predictions of the average ion concentrations are obtained from a one-dimensional model of the flow through the process chamber, and etch rate uniformity is predicted with a two-dimensional process chamber model.

We are able to predict radial etch-rate variation by combining the results of these models (Fig. 2). Results from the two-dimensional model show the importance of species diffusion in CDE reactors and the effect of processor geometry and inflow conditions on etch rate profiles. These calculations are being used to improve chamber designs to achieve uniform etching profiles.

This work is supported by Sandia National Laboratories under a CRADA with SEMATECH.

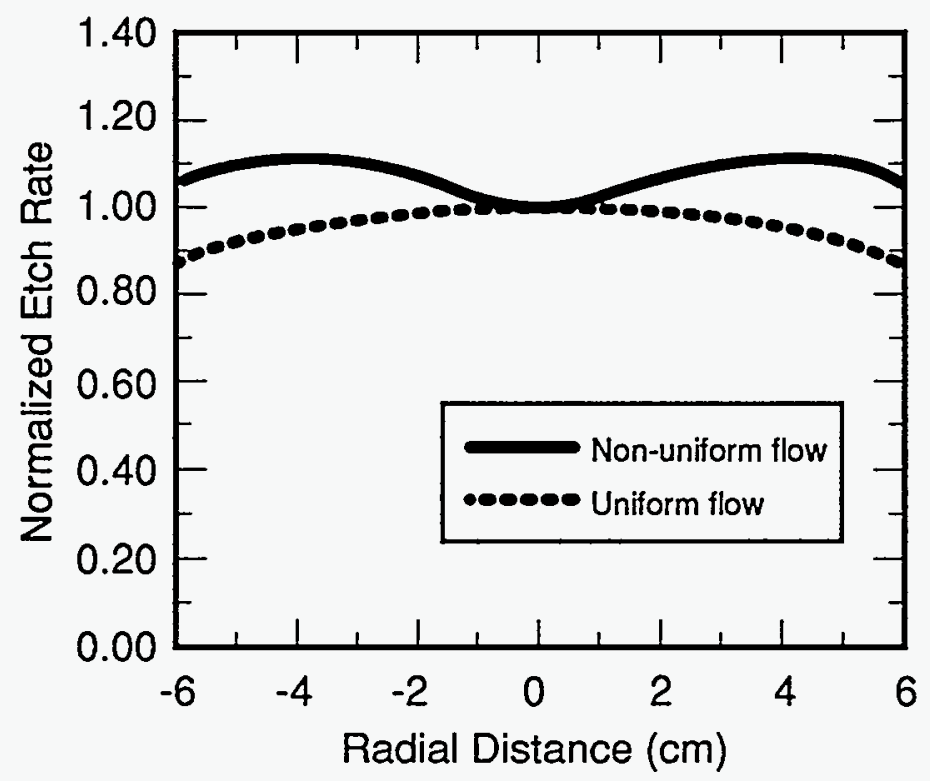

Figure 2. Results of 2-D reacting flow calculations through a typical CDE process chamber, including the effect of inlet flow uniformity and silicon etching by atomic fluorine on the wafer surface. The figure shows predicted silicon etch rate profiles as a function of inlet condition. 


\title{
Hydrothermal Oxidation Kinetics of Methanol
}

\author{
An optically accessible flow reactor is used in conjunction with Raman spectroscopy to \\ measure the kinetics of methanol oxidation over a range of conditions. Recently developed \\ elementary reaction models show good agreement with the experimental results.
}

\section{S. F. Rice, R. G. Hanush, and R. R. Steeper}

Supercritical water oxidation (SCWO) is a technology under development by government laboratories, universities, and private industry for the treatment of aqueous wastes. However, the current understanding of the rates and mechanisms of reactions in supercritical water is restricted to a handful of empirical mechanisms for very simple chemicals. These mechanisms are of limited use in the formulation of predictive models of SCWO that will be needed in the future for the design and operation of large-scale waste processing equipment. To be generally applicable as design tools, models must be based on elementary reaction steps or at least on a detailed quantitative mechanistic description incorporating all the key fundamental reactions.

We are developing an experimental data base, using an optically accessible flow reactor, for evaluating the details of methanol oxidation. Our experimental results are compared to the predictions from an elementary reaction step model that has been developed for these high-density hydrothermal conditions. The mechanistic details of the oxidation of methanol in supercritical water serve as important building blocks in developing useful models for the destruction of hazardous waste by supercritical water oxidation.

Sandia's optically accessible supercritical water reactor consists of two feed lines that preheat oxidizer and fuel to reaction conditions. These two lines are joined at a tee to generate a single line that incorporates a high-pressure, high-temperature optical cell to allow a direct view of the reacting flow. The reacting flow is maintained at near isothermal conditions by a series of individually controlled cable heaters helically wrapped around the tubing to overcome losses due to imperfect insulation. The lines can be cooled to remove heat generated from the oxidation reaction. The cell has three sapphire windows positioned at $90^{\circ}$ relative to each other to provide for a laser beam probe and the collection of Raman scattered light. The Raman spectrum of individual species present in the reaction, when calibrated, is used as a diagnostic to identify the absolute concentration of that particular compound. In addition to monitoring the initial feed material, Raman signals due to stable intermediates including formaldehyde and carbon monoxide have been identified. By varying the position of the cell relative to the mixing point along with varying the reactant flow rates, reaction times ranging from $0.15 \mathrm{~s}$ to $10 \mathrm{~s}$ can be probed in the same apparatus.

We have examined the oxidation of methanol by oxygen in supercritical water over the temperature range from $440^{\circ} \mathrm{C}$ to $500{ }^{\circ} \mathrm{C}$ and residence times from $0.17 \mathrm{~s}$ to $3.0 \mathrm{~s}$. A subset of the results from these measurements at an equivalence ratio of $\Phi=$ 0.5 is shown in Figure 1. The initial methanol concentration was 0.5 moles/liter at ambient conditions, which corresponds to approximately 0.05 moles/liter at reaction conditions, depending on experimental temperature. Oxygen was added at a varying equivalence ratios to complete the experimental set.

The Raman spectroscopic diagnostic has allowed us to collect a large set of data to compare with predictive models. In collaboration with researchers at MIT, LLNL, and The University of Iowa $[1,2]$, we have developed an elementary reaction model that is in good agreement with the observed rate of methanol consumption over this temperature 


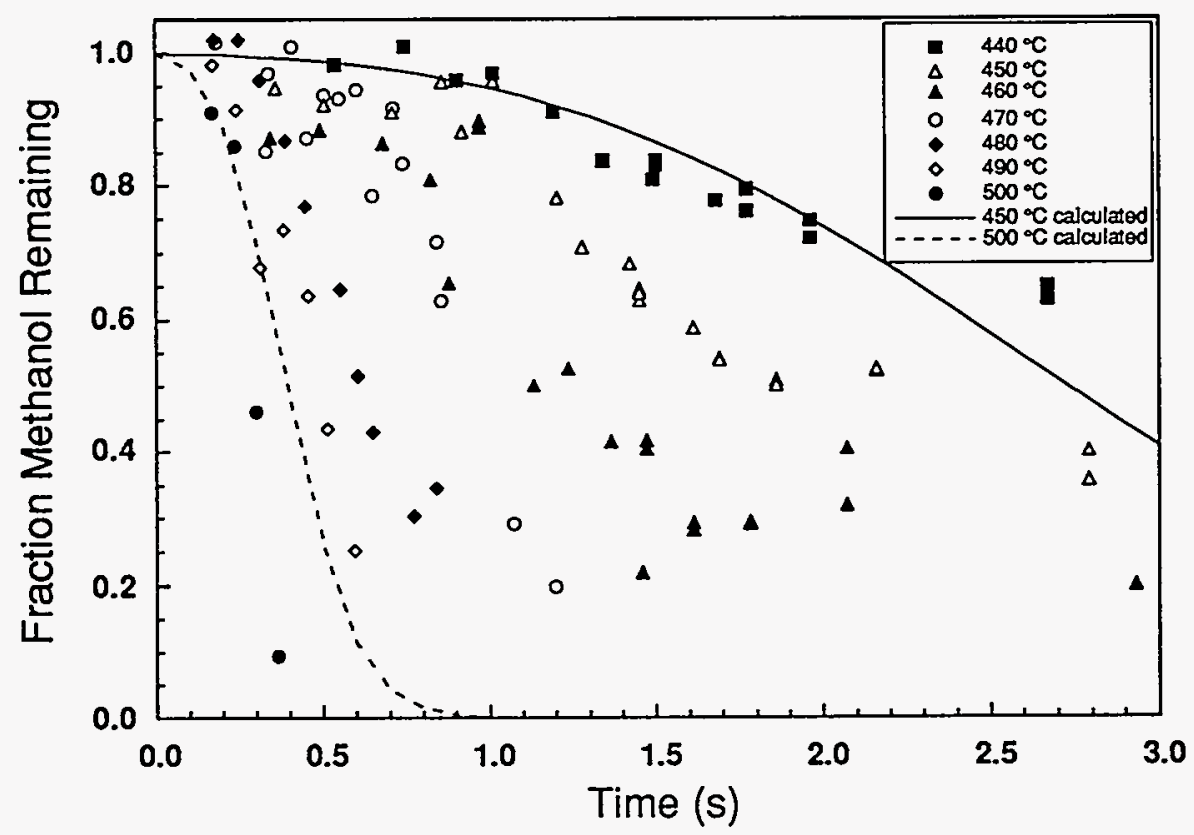

Figure 1. Plot of all the data collected on the oxidation of methanol between $440^{\circ} \mathrm{C}$ and $500^{\circ} \mathrm{C}$. Included on the plot are the curves predicted by the model for $450^{\circ} \mathrm{C}$ and $500^{\circ} \mathrm{C}$ at the same conditions as the experiment.

range. The model appears to be slightly underpredicting the conversion at a given temperature.

In addition to testing the model's ability to represent the loss of initial fuel, we have examined how well it represents the production of formaldehyde, a key intermediate. The concentration of formaldehyde in the reaction mixture is predicted by the elementary model to be as high as $15 \%$ of the initial feed concentration at $500^{\circ} \mathrm{C}$ and $0.3 \mathrm{~s}$ into the reaction. The result observed using the in situ Raman spectroscopic diagnostic is in good agreement with this value.

These results show that the present model for the oxidation of methanol can represent the important aspects of the oxidation process. We intend to extend this modeling and experimental effort to several more complicated alcohols, including 2-propanol and eventually phenol.

This work was supported by the multiagency DoD/DOE/EPA Strategic Environmental Research and Development Program (SERDP).

\section{References}

[1] Schmitt, R. G., Butler, P. B., Bergan, N. E., Pitz W. J., Westbrook, C. K. "Destruction of Hazardous Waste in Supercritical Water II: A Study of High-Pressure Methanol Oxidation Kinetics;" presented at the Fall Meeting of the Western States Section of the Combustion Institute., Los Angeles, October 13-15, 1991.

[2] Webley, P. A., and Tester, J. W. "Fundamental Kinetics of Methanol Oxidation in Supercritical Water." In Supercritical Fluid Science and Technology; Johnston, K. P., Penninger, J. M. L., Eds.; ACS Symposium Series 406; American Chemical Society: Washington, DC, 1989; pp 259-275. 


\title{
Destruction of Hazardous Munitions by Supercritical Water Oxidation
}

\author{
Supercritical water oxidation has the potential to be an affordable and environmentally \\ friendly way to dispose of a variety of hazardous obsolete munitions including military dyes \\ and an assortment of pyrotechnics such as colored smokes and flares. The results from \\ feasibility testing of dyes and pyrotechnics in the CRF's Materials Evaluation Reactor \\ illustrate this potential by determining suitable operating conditions and identifying large- \\ scale system design strategies.
}

\section{S. F. Rice, C. A. LaJeunesse, R. G. Hanush, and J. D. Aiken1}

The U. S. military stockpile has large quantities of obsolete munitions awaiting disposal. Although suitable means for the safe dismantlement of much of this stockpile have been identified, there are still quantities of specialty materials for which existing methods have been deemed inappropriate from an environmental standpoint. Among these munitions are colored spotting dyes and pyrotechnic colored smoke compositions. In open air burn or incineration treatment processes these materials produce large quantities of toxic, and possibly carcinogenic, gases and particulate matter.

The U. S. Army Armament Research, Development, and Engineering Center (ARDEC) is interested in developing alternative treatment methods for the final disposal of these materials that do not have the problems of open air burning or incineration. Consequently, a series of tests was undertaken at the Combustion Research Facility to assess the feasibility of using supercritical water oxidation (SCWO) to effectively destroy the organic dyes in these munitions. We have processed four dyes in the CRF's SCWO flow reactor, evaluated experimental destruction efficiencies as a function of temperature, and identified several operational considerations for pilot plant scale-up.

Supercritical water oxidation is conceptually simple. Aqueous waste, typically ranging from $1-10 \mathrm{wt} \%$ oxidizable material, is

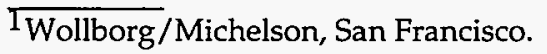

pressurized and heated to supercritical conditions (above $374^{\circ} \mathrm{C}$ and $22.1 \mathrm{MPa}$ ) where it can function as a fuel in an oxidation reaction. An oxidizer is added and the elevated temperature of the mix, typically at $550^{\circ} \mathrm{C}$, is maintained primarily by the excess heat of reaction. Given adequate reaction time, the waste-fuel is converted to relatively innocuous products; organic carbon in the feed emerges as $\mathrm{CO}_{2}$, nitrogen is converted to $\mathrm{N}_{2}$, and metals, heteroatoms, and halides appear in the effluent as inorganic salts and acids. The results from these dye processing tests provide a guide to the design of SCWO equipment by establishing the reaction conditions necessary to effectively destroy the dyes. Process difficulties can be identified so that they can be addressed prior to designing and building a prototype reactor.

The SCWO Materials Evaluation Reactor is constructed from Inconel 625 and is approximately $750 \mathrm{~cm}$ long. It is designed to maintain isothermal conditions over a length of $490 \mathrm{~cm}$ following mixing of the reactants and afford experimental residence times from 0.15 to $20 \mathrm{~s}$.

Testing was done in one of two different modes with one used for temperaturedependent measurements conducted on the orange dye only. The second method was instituted as a result of the dye pyrolysis and charring that occurred using the first method. The principal difference between the two methods is that in first experimental strategy, the reactants are preheated separately, prior to mixing, while in the second strategy the 
reactants are mixed first and then heated. The procedure was as follows: 1) a solution of the dye was prepared in water at a nominal concentration of $0.5 \mathrm{wt} \%, 2)$ the solution was pressurized to $27.2 \mathrm{MPa}(4000 \mathrm{psi})$ and heated to temperature in a series of tube furnaces, 3 ) in a parallel line, a solution of $5 \mathrm{wt} \%$ hydrogen peroxide in water was pressurized and brought to reaction temperature (when the peroxide was heated to above $400^{\circ} \mathrm{C}$, it rapidly decomposed to molecular oxygen and water), 4) these two solutions were mixed at known flow rates, temperature, and pressure and allowed to react in a fixed length of tubing, 5) the mixture was then cooled rapidly and diverted to the sample collection vessel for liquid effluent analysis. The procedure for the second method is identical to the first except the two solutions were mixed before heating rather than heated separately.

Orange $\mathrm{G}$ dye, $\mathrm{Na}_{2}\left(\mathrm{C}_{16} \mathrm{H}_{10} \mathrm{~N}_{2} \mathrm{O}_{7} \mathrm{~S}_{2}\right)$ was processed in the flow reactor at constant flow rate and oxidizer concentration; only temperature was varied. The samples were analyzed for input and effluent total organic carbon (TOC). The results are presented in Table 1. At temperatures above $550^{\circ} \mathrm{C}$ the orange dye is destroyed effectively. The TOC that remains in the effluent at these temperatures is probably composed of relatively stable low molecular weight organic species such as methanol, formic acid, and formaldehyde or single ring aromatics such as phenol. Carbonaceous deposits were formed in the dye feed line due to pyrolysis in the absence of oxygen.

Using the second method, the dyes are destroyed to $>99 \%$ without the pyrolysis problems. Analysis of the primary hazardous components, the specific dye molecules, using spectrophotometry indicated that all dyes, except the green dye, are destroyed below the detection limit of the technique, $0.5 \mathrm{ppm}$. The pale yellow appearance present in the effluent from green-dye processing is probably chromate ion from mild corrosion of the reactor.

Although at temperatures above $550^{\circ} \mathrm{C}$ the dyes and partial oxidation products were destroyed to a minimum of $99.5 \%$ DRE in 10 seconds, destruction of the toxic components of the dyes resulted in plugging and fouling of the reactor and general (leaching) corrosion from the reactor tubing. When the organic dye was heated above $350^{\circ} \mathrm{C}$ before introduction of oxidant, it pyrolized, adhered and fouled the reactor until flow restriction became serious. We did not find a method to remove the pyrolytic material other than a mechanical honing operation. Mixing the oxidant with the waste prior to heating eliminated the carbonaceous deposits, but produced "sticky" salts that agglomerated on the walls of the reactor vessel until a shut-down was initiated by a rise in pressure upstream of the plug. Examination indicated that this material was anhydrous sodium sulfate, $\mathrm{Na}_{2} \mathrm{SO}_{4}$.

This feasibility study has led to the development of a new reactor design now being developed at Sandia in conjunction with GenCorp Aerojet and Foster Wheeler Development Corporation that is likely to eliminate the salt deposition and corrosion problems encountered in these initial tests.

\begin{tabular}{|c|c|c|c|c|}
\hline \multicolumn{3}{|c|}{$\begin{array}{l}\text { Analytical Method: } \\
\text { Oxidizer: } \\
\text { Flow Rate: } \\
\text { Amount Processed: } \\
\text { Input Concentration: } \\
\text { Appearance: }\end{array}$} & \multicolumn{2}{|c|}{$\begin{array}{l}\text { Total Organic Carbon } \\
5 \mathrm{wt} \% \text { hydrogen peroxide } \\
0.75 \mathrm{~g} / \mathrm{sec} \\
5.7 \mathrm{liters} \\
2140 \mathrm{ppm} \text { TOC, } 0.5 \mathrm{wt} \% \text { dye } \\
\text { dark orange }\end{array}$} \\
\hline$\left({ }^{\circ} \mathrm{C}\right)$ & $\begin{array}{l}\text { TOC } \\
\text { (ppm) }\end{array}$ & $\begin{array}{l}\text { Residence } \\
\text { Time (s) }\end{array}$ & $\underset{\%}{\mathrm{DRE}}$ & Appearance \\
\hline 556 & 3.35 & 7.4 & 99.69 & colorless \\
\hline 553 & 3.39 & 7.4 & 99.68 & colorless \\
\hline 542 & 4.51 & 76 & 99.58 & colorless \\
\hline 520 & 21.4 & 8.1 & 98.00 & pale yellow \\
\hline 506 & 180 & 8.5 & 83.1 & brown \\
\hline 484 & 247 & 9.2 & 76.9 & brown/solids \\
\hline
\end{tabular}

Table 2. Destruction Efficiencies for Four Dyes.
Oxidizer:
Flow Rate: 5 wt\% hydrogen peroxide Amount Processed: Input Concentration: Temperature

$$
0.6 \mathrm{~g} / \mathrm{sec}
$$
3.7-7.5 liters/dye
$\sim 0.25 \mathrm{wt} \%$ dye
$560^{\circ} \mathrm{C}$

$\begin{array}{ccccc}\text { Sample } & \begin{array}{c}\text { TOC }_{\mathrm{O}} \\ (\mathrm{ppm})\end{array} & \begin{array}{c}\mathrm{TOC}_{\mathrm{i}} \\ (\mathrm{ppm})\end{array} & \text { DRE } & \text { Appearance } \\ \text { Orange } & 2.36 & 780 & 99.69 & \text { colorless } \\ \text { Red } & 4.11 & 936 & 99.56 & \text { colorless } \\ \text { Blue } & 3.74 & 1232 & 99.69 & \text { colorless } \\ \text { Green } & 2.65 & 819 & 99.67 & \text { yellow }\end{array}$

This work was supported by the U.S. Army Armament Research Development and Engineering Center at Picatinny Arsenal. 


\title{
Optical Monitoring Of the Oxidation of Methane Under Supercritical Water Conditions
}

\author{
We are using Raman spectroscopy to make in situ measurements of the oxidation rate of \\ methane under supercritical water conditions. The results are contributing to the \\ development of a new waste treatment technology, supercritical water oxidation.

\section{R. R. Steeper, S. F. Rice, and J. D. Aiken'1}

Supercritical water oxidation (SCWO) is a developing technology for the destruction of a wide range of organic hazardous wastes. This process oxidizes wastes in the presence of a large fraction of water at conditions above the thermodynamic critical point of water, that is, at high pressure (27.5 $\mathrm{MPa}$, around $4000 \mathrm{psi}$ ) and moderate temperatures (between 450 and $600^{\circ} \mathrm{C}$ ). The novel process achieves high destruction efficiencies in a compact system that lends itself to the environmentally safe treatment of many DOE, DoD, industrial, and municipal wastes.

The successful development of this technology depends on understanding the reaction chemistry of compounds at SCWO conditions. Predictive chemistry models, as they become available, will play an important role as design and operational tools for commercial-scale processes. We are currently conducting experiments to examine the oxidation kinetics of methane (a relatively difficult compound to destroy) in supercritical water. Our near-term goal is to obtain a simplified global expression for its reaction rate applicable at the conditions of commercial-scale SCWO processes. Our longer term goal is the development of a many-step, elementary reaction mechanism that will facilitate prediction of rates for a wide range of candidate wastes. To achieve this, we need measurements, not only of fuel disappearance rates, but also of production and consumption rates of key intermediates.

Our experiments are conducted in an optically accessible, constant-volume reactor permitting the continual, in-situ measurement of species concentrations using spontaneous Raman spectroscopy. In experiments to date, we have measured methane, oxygen, carbon

\footnotetext{
${ }^{1}$ Wollborg/Micheison, San Francisco
}

dioxide, and carbon monoxide concentration histories for the following range of operating conditions. Initial methane concentrations were chosen high enough to mimic commercial-scale processes (> 1 mole\%); equivalence ratios ranged from lean to rich; the temperature range (determined by experimental constraints) was near process temperature $\left(390\right.$ to $\left.440^{\circ} \mathrm{C}\right)$; and both "high" (27.5 $\mathrm{MPa}$, about $4000 \mathrm{psi}$ ) and "low" (13.5 $\mathrm{MPa}$ ) pressures were used to enable determination of reaction rate pressure dependency.

Typical data from a single experiment are presented in Figure 1. At time zero, an oxygen/nitrogen mixture is injected into a homogeneous mixture of methane and supercritical water. The figure shows the subsequent consumption of methane and oxygen, the production of carbon dioxide, and the production followed by consumption of carbon monoxide.

The more than 500 data points from our 15 high pressure experiments were fit to the global rate expression:

$$
\mathrm{d}\left[\mathrm{CH}_{4}\right] / \mathrm{dt}=-\mathrm{k}\left[\mathrm{CH}_{4}\right]^{\mathrm{a}}\left[\mathrm{O}_{2}\right]^{\mathrm{b}} \text {, }
$$

where $k=A \exp \left(-E_{a} / R T\right)$. Four fit parameters were obtained: a pre-exponential factor, A; an activation energy $E_{a} ;$ and reaction orders with respect to methane and oxygen, $a$ and $b$. These four values serve to predict closely all the data over the full range of operating conditions, as illustrated by the close fit of the curve to the data points in Figure 1. We obtained a similar set of four fit parameters for our 14 low pressure experiments.

Little physical significance can be attached to individual fit parameters since the one-step global mechanism used cannot represent the 


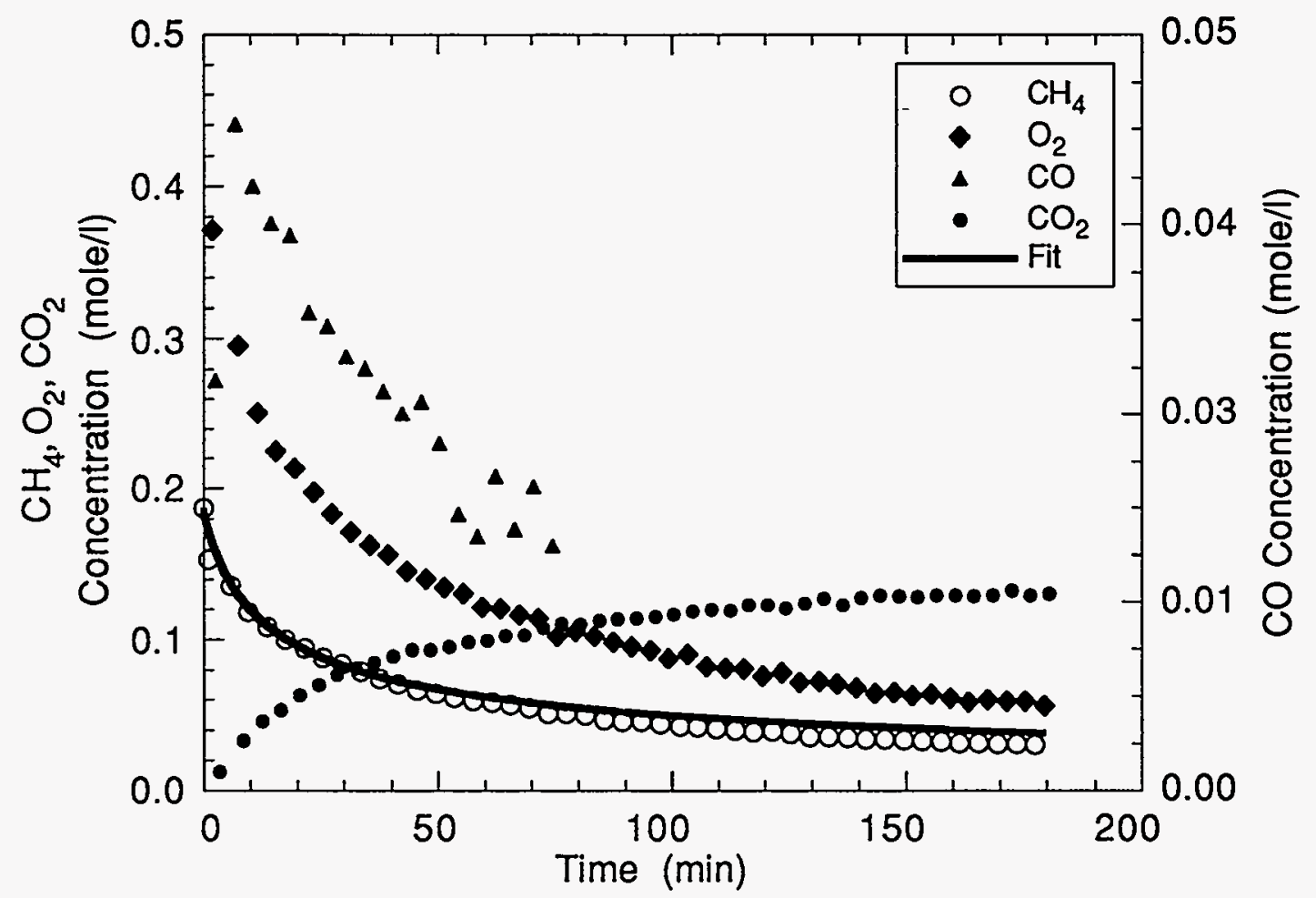

Figure 1. Typical experimental data (symbols) at $27.5 \mathrm{MPa}, 391{ }^{\circ} \mathrm{C}$, and stoichiometric methane/oxygen ratio. The solid line represents methane concentration predictions using the four fit parameters.

large number of actual elementary reactions that contribute to the overall rate. On the other hand, the global fit provides a means of comparing results from different experiments, and can be used to predict methane reaction rates for conditions within the range of these experiments - a useful tool for process modeling.

The literature contains other reports of SCWO kinetics measurements performed in fundamentally different reactors. In these reports, the type of reactor used limited initial methane concentration to values two ordersof-magnitude lower than the Sandia reactor. It is not surprising, then, that the global fit parameters do not agree. For example, the current study determined a reaction order with respect to methane close to 2 , while previous studies at lower initial concentrations yielded values of unity. The new data emphasize that it is inappropriate to take global rate expressions obtained at lower concentrations and extrapolate them to concentrations representative of commercial-scale SCWO processes. On the other hand, when we performed a combined fit using both our data and previous data, we obtained a global model that predicts methane concentrations within $10 \%$ over the combined wide range of initial concentrations and temperatures.

A surprising result emerged from the comparison of reaction rates at high and low pressures in these initial experiments. For comparable temperatures and initial methane and oxygen concentrations, measured rates are significantly higher at $13.5 \mathrm{MPa}$ than at $27.5 \mathrm{MPa}$. The effect may be chemical in origin and tied to the participation of water molecules in the key reaction steps. Transport effects could also be responsible if diffusion rates become slow enough at higher pressure so that key reactions become diffusion-limited. Further experiments to investigate these issues are planned-for example, argon will be substituted for water to determine whether or not the same inverse-pressure dependence is observed.

This work was supported by the U.S. DoD/ DOE/EPA SERDP Program and by the U.S. DOE Office of Basic Energy Sciences, Engineering and Geosciences Division. 


\title{
Optical Spectroscopy Applied to Process Control in Basic Oxygen Steelmaking
}

\author{
Optical absorption and emission spectroscopies are used to monitor gas-phase molecular \\ concentrations and temperatures during basic oxygen steelmaking.
}

\section{S. W. Allendorf, D. K. Ottesen, M. Bonin1, K. Chen 2, H. A. Johnsen, D. Jung ${ }^{3}$, F. W. Kent, P. D. Stelts ${ }^{2}$, and J. Wang 3}

The basic oxygen furnace (BOF) converts molten iron and scrap metal into steel in a batch process that oxidizes carbon and impurities with a high-velocity jet of oxygen. The development of optical sensors for commercial steelmaking offers the possibility of real-time process control in hostile, hightemperature environments by means of remote, non-contacting measurements. Current BOF technology relies on a materials balance for calculating the necessary quantities of reactants and processing times. We are developing novel optical sensors which are designed to provide real-time, on-line analysis of the off-gases released from the melt in the BOF during a steelmaking heat.

Gas-phase sensors are promising for BOF control for a number of reasons. The ratio of $\mathrm{CO}$ to $\mathrm{CO}_{2}$ concentration in the off-gas has previously been shown to reflect the progress of decarburization in the bath. Determination of the off-gas temperature is necessary to close the energy balance and to carefully control the post-combustion of carbon monoxide to maximize the heat transfer into the bath without overheating the refractory lining. Additional capabilities for measuring $\mathrm{H}_{2} \mathrm{O}$ are required to further characterize the off-gas process stream.

Through laboratory work at the CRF and pilot-scale experiments using a two-ton BOF facility at Bethlehem Steel's Homer Research Laboratory (HRL) in Bethlehem, PA, we are

\footnotetext{
${ }^{1}$ INSITEC Instruments Corporation.

2 Bethlehem Steel Corporation

${ }^{3}$ Cornell University
}

evaluating two line-of-sight optical techniques, direct absorption spectroscopy and emission spectroscopy, for determining the average line-of-sight concentration and temperature of infrared-active gases $(\mathrm{CO}$, $\mathrm{CO}_{2}, \mathrm{H}_{2} \mathrm{O}$ ) in the BOF off-gas. The use of these techniques for measurements in the steelmaking environment is advantageous in view of their simplicity, robust instrumentation, long-term reliability, and relatively low cost.

The first approach involves tunable diode laser (TDL) absorption spectroscopy. By monitoring the relative intensities of the absorption features over the course of the heat, we measure temperature and $\mathrm{CO}$ concentration along the laser line-of-sight. Figure 1 presents a typical gas-phase mid-infrared absorption spectrum during BOF oxygen blowing (solid line), and a calculated $\mathrm{CO}$ absorption spectrum (dotted line.) Very good agreement is obtained for $\mathrm{CO}$ hot-band lines for a calculated temperature of $2125^{\circ} \mathrm{F}(1435 \mathrm{~K})$ and concentration of $12 \%$. In this wavelength region, $\mathrm{CO}$ accounts for most of the absorption features, but one particularly strong $\mathrm{CO}_{2}$ feature is indicated in the figure. The timehistory of the ratio of $\mathrm{CO}$ to $\mathrm{CO}_{2}$ is expected to be an indication of the completeness of decarburization. The $\mathrm{CO}$ to $\mathrm{CO}_{2}$ ratio and the measured gas temperature provide information about the effectiveness of the oxygen lance operation with respect to variables such as lance-height position and oxygen flow rate, and can be used for process control.

A second line-of-sight approach that we are developing involves the measurement of emission from infrared-active gas species. The optics of a spectrometer are focused on the 


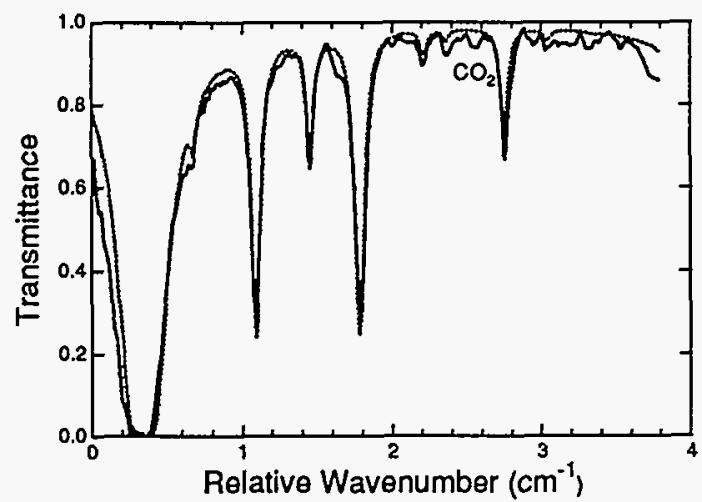

Figure 1. Plot of measured transmittance (solid curve) of the TDL through the off-gas of a pilotscale BOF. Comparison with CO transmittance spectrum calculated at $1435 \mathrm{~K}$ (dashed curve) indicates that the off-gas is predominantly composed of $\mathrm{CO}$ with lesser amounts of $\mathrm{CO}_{2}$ and $\mathrm{H}_{2} \mathrm{O}$.

water-cooled oxygen lance to enhance the spectral contrast of the gas-phase emitters and to limit the optical pathlength. The resulting spectra are monitored during the heat. A typical mid-infrared emission spectrum (solid curve) is presented in Figure 2, along with an FTIR transmission spectrum (dashed curve) obtained at the CRF using an oxygenacetylene burner. The evenly spaced lines in both spectra are due to ground state $\mathrm{CO}$ vibrational transitions $(v=1 \rightarrow v=0$.) We also observe weaker features due to $\mathrm{CO}$ hot-band transitions (e.g., $v=2 \rightarrow \mathrm{v}=1$.) The relative intensities of these lines will ultimately be used to calculate temperatures and concentrations in a fashion analogous to the results for the TDL absorption sensor described above. The offset from the baseline is due to broadband emission from particulates in the sensor field-of-view. This particulate emission does not interfere with the measurement of gas-phase emission lines in these pilot-scale tests.

We have recently completed a series of three field trials at HRL, during which we successfully tested both gas-phase sensors. The absorption sensor data consistently show that after an initial period of oxygen blowing, the $\mathrm{CO}$ concentration and temperature are rather stable. The absolute values of the concentration and temperature vary from heat to heat, depending on experimental conditions such as the BOF initial temperature, oxygen

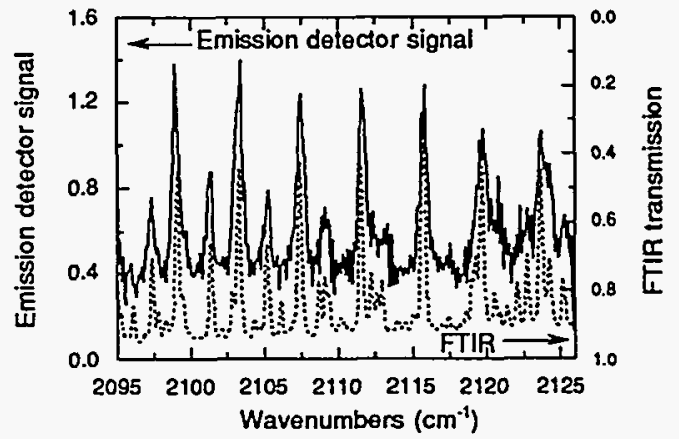

Figure 2. Comparison of emission sensor signal during BOF oxygen blowing (solid curve) and FTIR transmission spectrum for laboratory burner (dashed curve).

flow rate, oxygen lance height above the melt, and whether a one-hole or a three-hole oxygen lance is used. In all heats, we observe a clear increase in laser transmittance followed by a sudden drop in $\mathrm{CO}$ concentration and $\mathrm{CO} / \mathrm{CO}_{2}$ ratio. This decrease in the $\mathrm{CO}$ concentration indicates that the carbon in the melt has been depleted, which we interpret to be the carbon endpoint of the heat.

The emission sensor data are similarly consistent during the pilot-scale BOF heats. There is an initial increase in emission line intensity followed by a period of stable emission contrast, which we define as the intensity of the gas-phase emission relative to the particulate gray body emission. The emission contrast rapidly increases at the same time that the laser transmittance increases. This contrast peaks, then sharply falls, as the amount of $\mathrm{CO}$ in the off-gas decreases.

In summary, both off-gas sensors show promise as real-time monitors for BOF steelmaking. Future efforts will focus on implementation of the sensors in a commercial, full-scale BOF at Bethlehem Steel's Sparrows Point plant, with the ultimate goal of incorporating the real-time information from the sensors into the BOF process control system.

This work was supported by the U.S. DOE Office of Industrial Technologies and by the American Iron and Steel Institute. 


\section{Miniature Optical Sensor Developed for Control of Basic Oxygen Steelmaking}

The basic oxygen furnace is the workhorse of modern steelmaking operations, yet is expensive to operate and difficult to control. A team from the CRF is collaborating with Bethlehem Steel

Corporation and the American Iron and Steel Institute to develop a rugged process control sensor that performs both multi-spectral thermography and optical range finding in the harsh environment of full-scale production furnaces.

\section{R. H. Hurt, M. P. Bonin, ${ }^{1}$ E. A. Fuchs, P. D. Stelts, ${ }^{2}$ F. W. Kent, and A. J. Salmi}

The basic oxygen furnace (BOF) is the workhorse of modern steelmaking operations, yet is expensive to operate and difficult to control. The primary barrier to improved control is the lack of on-line process information on the properties of the steel melt. Melt measurements are difficult to make in situ because of the hostile environment, which includes high temperatures, corrosive liquids, and particle and droplet laden off-gases. There are very few locations that provide access to the melt phase as well as a good chance for longterm instrument survival.

Sandia has teamed with Bethlehem Steel Corporation and the American Iron and Steel Institute to develop a ruggedized optical sensor for improved control of BOFs. The concept is depicted in Fig. 1. A miniature sensor located within the water-cooled confines of the oxygen lance gathers spatiallyresolved visible and near-infrared emission from the region below the lance tip. The prototype sensor is an integrated device that makes three important process measurements: (1) "hot spot," or flame zone temperature during oxygen blowing, (2) post-blow bulk melt temperature, and (3) melt surface position and furnace bottom contour through laser reflectance range finding techniques.

To provide design data for the lance-based sensor, a remote-controlled, heat-shielded thermography unit was used to obtain monochromatic image sequences of melt surfaces in a full scale steelmaking ladle that

\footnotetext{
1 Insitec Measurement Systems, San Ramon, CA

${ }^{2}$ Bethlehem Steel Corporation, Bethlehem, PA
}

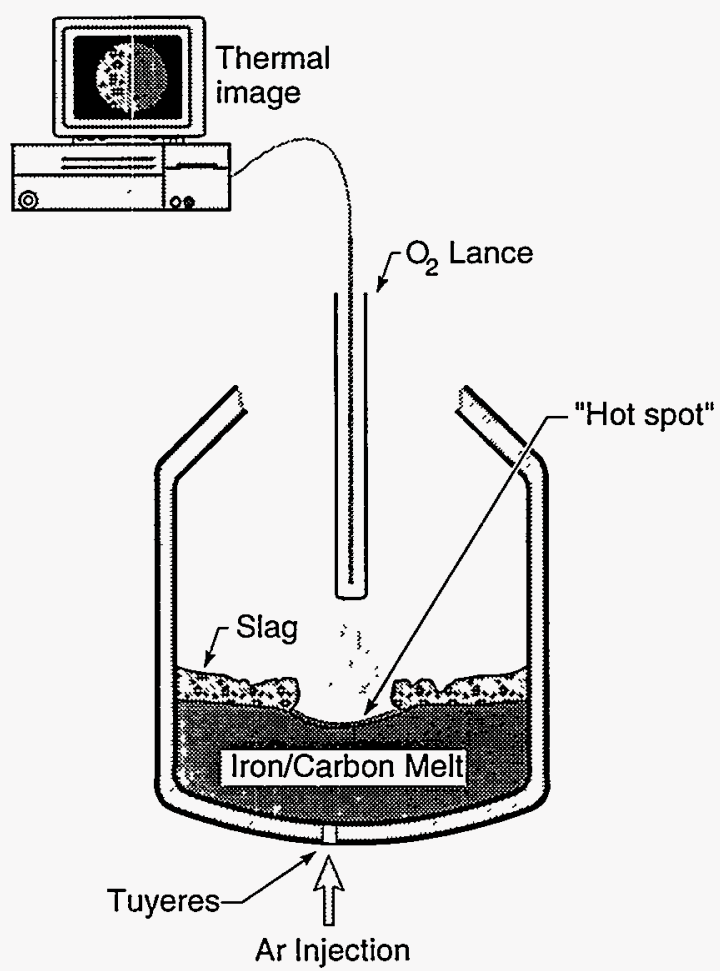

Figure 1. Lance-based optical sensing in the basic oxygen steelmaking process.

simulate the bath surface expected inside the BOF vessel. This experiment is depicted in Figure 2.

Figure 3 shows a typical monochromatic thermal image, in which a region of bare melt surface has been exposed by rising bubbles of argon gas introduced through the refractory furnace bottom. Distinctly visible is a turbulent melt "eye," a surrounding slag phase, and a line of flamelets at the melt/slag interface. 


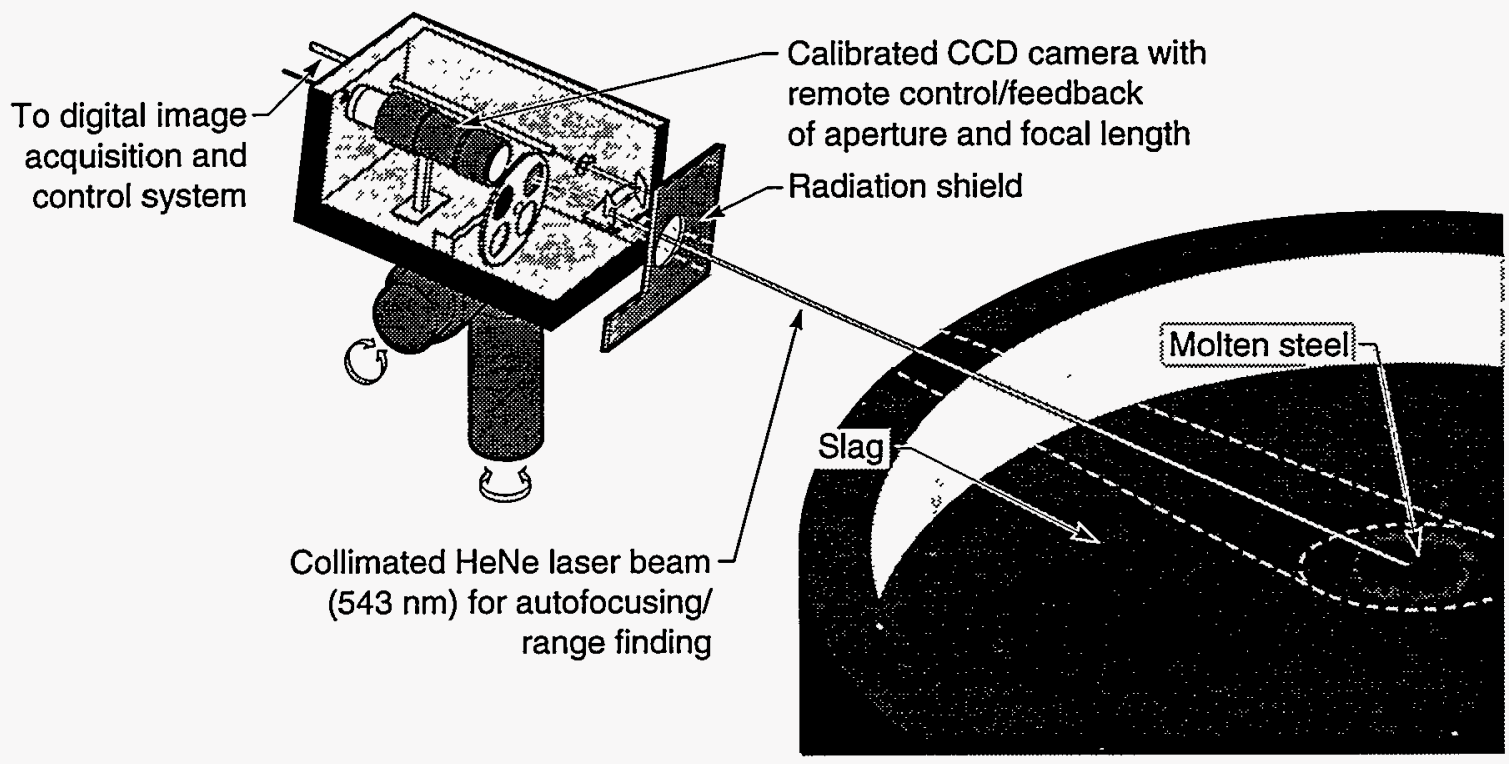

Figure 2. Experiment to characterize the optical properties of turbulent melt surfaces using a remotecontrolled, heat-shielded thermography unit in an operating commercial steel mill.

Light scattering and image obscuration by particulate matter is also present in some of the thermal images. On the melt surface itself, a characteristic optical texture is observed consisting of convex dark tiles with bright edge regions. Digital analysis of these images has revealed the origin of this tortoise shell optical structure and its implications for temperature measurement.

Because of the complex multi-phase nature of the region below the lance tip, imagebased techniques have been chosen to ensure proper interpretation of the optical signals. In the final process sensor, temperatures will be measured by on-line digital analysis of multispectral (multi-channel) thermal images. Several options are being actively pursued for incorporation of laser range finding capabilities.

Pilot-scale work to date has led to an initial design for a full-scale, lance-based sensor package that will be tested at Bethlehem Steel's commercial production facilities in 1995.

This work was supported by the U.S. DOE Office of Energy Efficiency and Renewable Energy, Office of Industrial Technologies, and by the American Iron and Steel Institute.

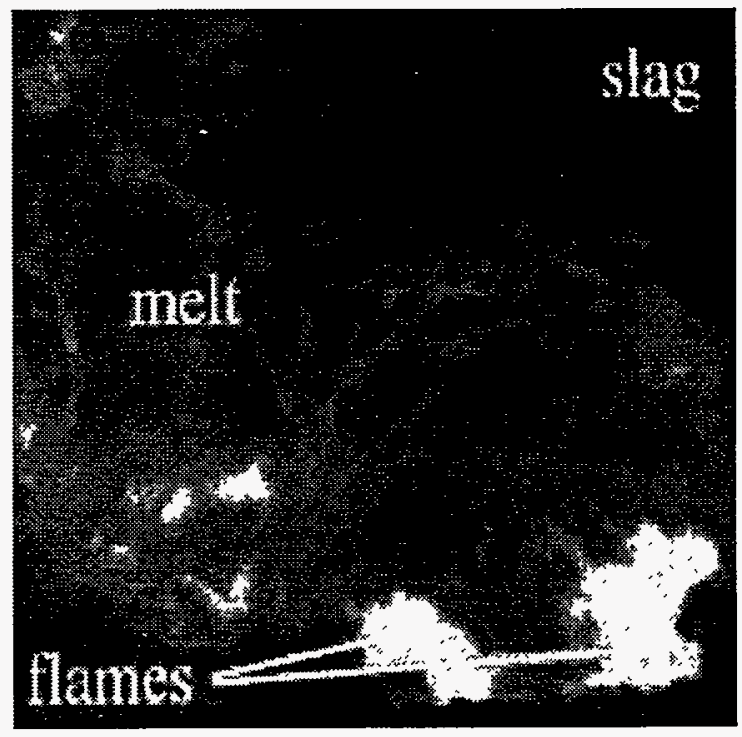

Figure 3. Monochromatic thermal image at 450 nm of a turbulent steel melt surface in a fullscale ladle. 


\section{Publications \\ 1994}

M. F. Abbott, R. E. Douglas, C. E. Fink, N. J. Deluliis, and L. L. Baxter, "A Modeling Strategy for Correlating Coal Quality to Power Plant Performance and Power Costs," J. Williamson and F. Wigley, eds., The Impact of Ash Deposition on Coal-Fired Plants, Taylor \& Francis, Washington, DC, 165-176.

M. D. Allendorf and R. H. Hurt, "A New Process for Low-Cost Production of Ceramic Composites," Materials Technology 9, 2.

J. Almlöf, P. Jensen, F. J. Northrup, C. M. Rohlfing, E. A. Rohlfing and T. J. Sears, "Comment on: The $\mathrm{n}_{1}+\mathrm{n}_{3}$ Combination Mode of $\mathrm{C}_{3}$ in Ar and Kr Matrices: Evidence for a Bent Structure," J. Chem. Phys. 101, 5413.

M. N. R. Ashfold, D. W. Chandler, C. C. Hayden, R. I. McKay and A. J. R. Heck, "Two-Colour Resonant-Enhanced Four-Wave Spectroscopy of Ammonia," Chem. Phys., in press.

W. T. Ashurst, "A Simple Illustration of Turbulent Flame Ball Growth," Combust. Sci. Technol. in press.

W. T. Ashurst, M. D. Checkel, and D. S.-K. Ting, "The Eddy Structure Model of Turbulent Flamelet Propagation, the Expanding Spherical and Steady Planar Cases," Combust. Sci. Technol. 99, 51.

R. S. Barlow and C. D. Carter, "Experiments on Nitric Oxide Formation in Turbulent Jet Flames," AIAA paper 94-0223.

R. S. Barlow and C. D. Carter, "Raman/Rayleigh/LIF Measurements of Nitric Oxide Formation in Turbulent Hydrogen Jet Flames," Combust. Flame 97, 261.

L. L. Baxter, R. E. Mitchell, and T. H. Fletcher, "Release of Inorganic Material During Coal Devolatilization," Combust. Flame, in press.

L. L. Baxter, "Development of Emission FTIR Spectroscopy as a Diagnostic for Ash Deposition During Coal Combustion," Combust. Flame, in press.

L. L. Baxter, "Release of Inorganic Material During Coal Devolatilization," Combust. Flame, in press.

L. L. Baxter, "In Situ, Real-Time Analysis of Ash Deposit Chemistry During Pulverized Coal Combustion," Energy \& Fuels, in press.

L. L. Baxter, T. R. Miles, Jr., B. M. Jenkins, G. R. Richards, and L. L. Oden, "Transformations and Deposition During Straw Combustion," Energy \& Fuels, in press.

L. L. Baxter, G. Richards and J. Harb, "Application of Advanced Technologies to Ash-Related Problems in Boilers," EPRI Conference on the Effects of Coal Quality in Power Plants, (Charleston, SC, August 17-19, 1994).

L. L. Baxter, "Nitrogen Release During Coal Combustion: Coal Rank and Extent of Combustion Trends," Energy \& Fuels, in press.

L. L. Baxter, and B. M. Jenkins, "Baseline NOx Emissions During Combustion of Wood-Derived Pyrolysis Oils," Specialists Workshop on Biomass Pyrolysis Oil Combustion, (Estes Park, CO, September 26-28, 1994). 
R. Behrens, T. Land, and S. Bulusu, "Thermal decomposition Mechanisms of Hexahydro-1-nitroso3,5-dinitro-s-triazine (ONDNTA)," Proceedings of the Nineteenth Army Science Conference, (Orlando, FL, June 1994).

R. Behrens, Jr. and S. Bulusu, "Thermal decomposition Reactions of HMX and RDX and their Importance in Predicting Cookoff Hazards," JANNAF Hazards Meeting, San Diego, CA, August 1, 1994.

S. E. Bisson and J. E. M. Goldsmith, "Measurements and Modeling of the Effect of Convective Clouds on the Upper Tropospheric Moisture Budget," Proceedings of Fourth Atmospheric Radiation Measurement (ARM) Science Team Meeting, (Charleston, SC, February 28-March 3, 1994), in press.

S. E. Bisson, "A Parametric Study of an Excimer-pumped, Nitrogen Raman Shifter for Lidar Applications," Appl. Opt., in press.

J. Brouwer, G. Sacchi, J. P. Longwell, A. F. Sarofim, and A. R. Kerstein, "A Turbulent Reacting Flow Model that Incorporates Detailed Chemical Kinetics," Combust. Sci. Technol., in press.

N. J. Brown, G. Liu, M. L. Koszykowski, "Functional Sensitivity Evaluation of Methane Combustion Mechanisms," Combust. Flame, in press.

M. N. Bui-Pham, A. Lutz, J. A. Miller, M. Desjardin, D. O'Shaughnessey, and R. Zondlak, "Rich Flammability Limits in $\mathrm{CH}_{3} \mathrm{OH} / \mathrm{CO} /$ Diluent Mixtures," Combust. Sci. Technol., in press.

S. Bulusu, R. Damavarapu, J. R. Autera, R. Behrens, Jr., L. M. Minier, J. Villanueva, K. Jayasuriya, and T. Axenrod, "Thermal Rearrangement of 1,4-Dinitroimidazole to 2,4-Dinitroimidazole: Characterization and Investigation of the Mechanism by Mass Spectrometry and Isotope Labeling," $J$. Phys. Chem., in press.

M. A. Buntine, D. W. Chandler, and C. C. Hayden, "Detection of Excited State Absorptions in Water via Laser-Induced Grating Spectroscopy," J. Chem. Phys., in press.

J. M. Card, J. H. Chen, M. Day, and S. Mahalingam, "Direct Numerical Simulations of Unsteady Nonpremixed Methane-Air Flames Modelled with Reduced Chemistry," Proceedings of the Stanford/NASA-Ames Center for Turbulence Summer Program, (Stanford, CA, 1994).

J. P. C. Chan, C. A. LaJeunesse, and S. F. Rice, "Experimental Techniques to Determine Salt Formation and Deposition in Supercritical Water Oxidation Reactors," 1994 International Mechanical Engineering Congress and Exposition, (Chicago, IL, November 6-11, 1994).

J. H. Chen and W. Kollmann, "Topological and Geometrical Properties of Turbulent Flames," Paper AIAA 94-0098, AIAA J., in press.

J. H. Chen, J. J. Lienau, and W. Kollmann, "Numerical Simulation of Low Reynolds Number Turbulence in Round Jets," Turbulent Shear Flows 9, ed. F. Durst (Springer-Verlag, Heidelberg, 1994), 141-152.

N. T. Clemens and P. H. Paul, "Effects of Heat Release on the Near-Field Flow Structure of Hydrogen Jet-Flames," Combust. Flame, in press.

N. T. Clemens and P. H. Paul, "Scalar Measurements in Compressible Axisymmetric Mixing Layers," Phys. Fluids $B$, in press. 


\section{Publications}

P. M. Danehy, P. H. Paul, and R. L. Farrow, "Temporal Evolution of Thermal Gratings in Degenerate Four-Wave Mixing," Conference on Lasers and Electro-Optics, Vol. 8, 1994 Technical Digest Series, (Optical Society of America, Washington, DC, 1994), 162-163.

K. A. Davis and R. H. Hurt, "Single Particle Experiments for the Investigation of Residual Carbon in Flyash," Pacific Rim International Conference on Environmental Control of Combustion Processes, (Maui, Hawaii, October 16-20, 1994).

K. A. Davis, R. H. Hurt, N. Y. C. Yang, and T. J. Headley, "Evolution of Char Chemistry, Crystallinity, and Ultra-Fine Structure During Pulverized-Coal Combustion," Combust. Flame (Twenty-Fifth Symposium (International) Issue), in press.

K. W. DeLong, D. J. Kane, and R. Trebino, "Comparison of Ultrashort Pulse Frequency-Resolved Optical Gating Traces for Three Common Beam Geometries," J. Opt. Soc. Am. B 11, 1595.

K. W. DeLong and R. Trebino, "Improved Ultrashort Pulse Retrieval Algorithm for FrequencyResolved Optical Gating," J. Opt. Soc. Am. A, 11, 2429.

K. W. DeLong, R. Trebino, J. Hunter, and W. E. White, "Frequency-Resolved Optical Gating Using Second-Harmonic Generation," J. Opt. Soc. Am. B 11, 2206.

K. W. DeLong, D. N. Fittinghoff, R. Trebino, A. Sullivan, J. Hunter, W. E. White, and D. J. Kane, "Frequency-Resolved Optical Gating: Measuring the Intensity and Phase of an Ultrashort Laser Pulse," Ultrafast Phenomena IX, ed. W. Knox (Springer-Verlag, 1994).

K. W. DeLong, D. N. Fittinghoff, R. Trebino, B. Kohler, and K. Wilson, "Pulse Retrieval in FrequencyResolved Optical Gating Using the Method of Generalized Projections," Opt. Lett., in press.

K. W. DeLong, C. L. Ladera, and R. Trebino, "Ultrashort Pulse Measurement Using NonInstantaneous Nonlinearities: Raman Effects in Frequency-Resolved Optical Gating," Opt. Lett., in press.

K. W. DeLong, "Measuring the Intensity and Phase of Two Ultrashort Laser Pulses on a Single Shot," in Generation, Amplification and Measurement of Ultrashort Pulses, ed. R. Trebino and I. A. Walmsley, (SPIE Press, Bellingham, 1994), 168.

J. R. Dunlop and E. A. Rohlfing, "Laser-Induced Gratings in Free Jets III. Saturation-Induced Anharmonic Gratings," J. Chem. Phys. 100, 856.

J. L. Durant, "Product Branching Fractions in the Reaction of NH(ND) ${ }^{3} \mathrm{~S}$ with NO," J. Phys. Chem. 98, 518.

J. L. Durant, Jr., "Reactions of $\mathrm{NH}_{\mathrm{X}}$ Species," Research in Chemical Kinetics, eds. G. Hancock and R. Compton, (Elsevier Science, Vol. 3, 1994), in press.

C. F. Edwards and K. D. Marx, "Classification of Steadiness in Sprays Using Ideal Spray Concepts," Seventh Annual Conference on Liquid Atomization and Spray Systems, (ILASS-Americas 94, Bellevue, WA, May 31-June 3, 1994).

C. Espey, J. E. Dec, T. A. Litzinger and D. A. Santavicca, "Planar Laser Raleigh Scattering for Quantitative Vapor-Fuel Imaging in a Diesel Jet," Combust. Flame, in press. 
R. L. Farrow, P. H. Paul, E. J. Friedman-Hill, and P. M. Danehy, "Degenerate Four-Wave Mixing for Quantitative Diagnostic Measurements," in Laser Applications to Chemical Analysis, 1994 Technical Digest Series, Vol. 5 (Optical Society of America, Washington, DC, 1994), 240-243.

D. N. Fittinghoff, K. W. DeLong, R. Trebino, and C. L. Ladera, "Noise Sensitivity in FrequencyResolved Optical Gating Measurements of Ultrashort Pulses," J. Opt. Soc. Am. B, in press.

J. T. Fourkas, L. Dhar, K. A. Nelson, and R. Trebino, "Spatially-Encoded, Single-Shot Ultrafast Spectroscopies," J. Opt. Soc. Am. B, in press.

E. J. Friedman-Hill, L. A. Rahn, and R. L. Farrow, "On the Interpretation and Rotational Assignment of Degenerate Four-Wave Mixing Spectra: Four-Photon Line Strengths for Crossover Resonances

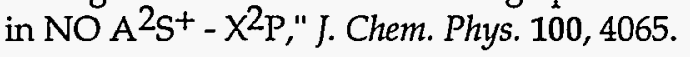

M. R. Furlanetto, J. W. Thoman, J. A. Gray, P. H. Paul, and J. L. Durant, Jr., "Near-Resonant Electronic Energy Transfer in Electronic Quenching of $\mathrm{NO}\left(\mathrm{A}^{2} \mathrm{~S}^{+}\right)$by Hydrocarbons and Ammonia," J. Chem. Phys. 101, 10452.

M. R. Furlanetto, J. W. Thoman, J. A. Gray, P. H. Paul, and J. L. Durant, Jr., "Near-Resonant Electronic Energy Transfer in the Electronic Quenching of NO $\mathrm{A}^{2} \mathrm{~S}^{+}$by Hydrocarbons and Ammonia," $J$. Chem. Phys., in press.

G. J. Germann and D. J. Rakestraw, "Multiplex Spectroscopy: Determining the Transition Moments and Absolute Concentrations of Molecular Species," Science 264, 1750.

G. J. Germann, R. L. Farrow and D. J. Rakestraw, "Infrared Degenerate Four-Wave Mixing Spectroscopy of Polyatomic Molecules: $\mathrm{CH}_{4}$ and $\mathrm{C}_{2} \mathrm{H}_{2}$, J. Opt Soc. Am. B, in press.

P. Glarborg, K. Dam-Johansen, J. A. Miller, and R. J. Kee, "Modeling the Thermal De-NO Process in Flow Reactors: Nitrous Oxide Formation and Surface Effects," Int. J. Chem. Kin. 26, 421.

P. Glarborg and J. A. Miller, "Mechanism and Modeling of Hydrogen Cyanide Oxidation in a Flow Reactor," Combust. Flame 99, 475.

J. E. M. Goldsmith, M. Lapp, S. E. Bisson, s. H. Melfi, D. N. Whiteman, R. A. Ferrare, and K. D. Evans, "Laser Remote Sensing of Water Vapor: Raman Lidar Development," Proceedings of the Third Atmospheric Radiation Measurement (ARM) Science Team Meeting (CONF-9303112, March 1994), 303-306.

J. E. M. Goldsmith, S. E. Bisson, and F. H. Blair, "Implementation of Raman Lidar for Profiling of Atmospheric Water Vapor and Aerosols at the SGP CART Site," Proceedings of Fourth Atmospheric Radiation Measurement (ARM) Science Team Meeting, (Charleston, SC, February 28-March 3, 1994) in press.

J. E. M. Goldsmith and S. E. Bisson, "Daytime Raman Lidar Profiling of Atmospheric Water Vapor," Proceedings of Seventeenth International Laser Radar Conference; Abstracts of Papers, (Sendai, Japan, July 25-29, 1994), 134-136.

J. E. M. Goldsmith and S. E. Bisson, "Raman Lidar Profiling of Atmospheric Water Vapor: Simultaneous Measurements with Two Collocated Systems," Bull. Amer. Meteor. Soc., 75, 975-982. 


\section{Publications}

J. E. M. Goldsmith and S. E. Bisson, "Measurements of Daytime and Upper Tropospheric Water Vapors by Raman Lidar," Optical Remote Sensing of the Atmosphere Technical Digest, (Optical Society of America, Washington, DC, 1995) in press.

C. C. Hayden and D. W. Chandler, "Femtosecond Time-Delayed Photoionization Studies Ultrafast Internal Conversion in 1,3,5-Hexatriene," J. Phys. Chem., in press.

R. H. Hurt, K. A. Davis, N. Y. C. Yang, J. Gibbins, and H. Valia, "Carbon Burnout in Pulverized Coal Combustion: An Overview of Mechanisms and Trends," EPRI Conference on the Effects of Coal Quality in Power Plants, (Charleston, SC, August 17-19, 1994).

R.H. Hurt and J. R. Gibbins, "Residual Carbon from Pulverized Coal Fired Boilers 1: Size Distribution and Combustion Reactivity," Fuel, in press.

R. Hurt, K. A. Davis, N. Y. C. Yang, T. J. Headley, and G. D. Mitchell, "Residual Carbon from Pulverized Coal Fired Boilers 2: Morphology and Physicochemical Properties," EPRI Conference on the Effects of Coal Quality in Power Plants, (Charleston, SC, August 17-19, 1994).

J. M. Card, R. Ryden, and F. A. Williams, "Influences of Flame-Vortex Interactions on Formation of Oxides of Nitrogen in Curved Methane-Air Diffusion Flamelets," Combust. Flame, in press.

S. J. Jacobs, K. T. Gillen, P. A. Cahill, C. C. Henderson and C. M. Rohlfing, "Theoretical and Experimental Investigations of Fullerene Derivatives: $\mathrm{C}_{60} \mathrm{H}_{2}, \mathrm{C}_{70} \mathrm{H}_{2}$ and $\mathrm{C}_{60}\left(\mathrm{CH}_{2}\right) 2, "$ Mat. Res. Soc. Symp. Proc. 349, 145 (1994).

B. M. Jenkins and L. L. Baxter, "Uncontrolled Pollutant Emissions from Biomass Combustion Under Simulated Boiler Furnace Conditions," Pacific Rim International Conference on Environmental Control of Combustion Processes, (Maui, Hawaii, October 16-20, 1994).

B. M. Jenkins, L. L. Baxter, T. R. Miles, Jr., L. L. Oden, R. W. Bryers, E. Winther, "Composition of Ash Deposits in Biomass Fueled Boilers: Results of Full-Scale and Laboratory Simulations," ASAE, Paper No. 946007, St. Joseph, MI (1994).

D. J. Kane, A. J. Taylor, R. Trebino, and K. W. DeLong, "Single-Shot Measurement of the Intensity and Phase of Femtosecond UV Laser Pulse Using Frequency-Resolved Optical Gating," Opt. Lett. 19, 1061.

R. J. Kee, E. Meeks, J. A. Miller, T. Takeno, M. Nishioka, and R. W. Dibble, "The Practical Exploitation of Strained Laminar Premixed Flames," Prog. Energy. Comb. Sci., in press.

A. R. Kerstein, R. W. Schefer and M. Namazian, "A Conditional Similarity Concept for Turbulent Shear Flow, With Application to Mixing in a Round Jet," Phys. Fluids 6, 642.

A. R. Kerstein and P. A. McMurtry, "Mean-Field Theories of Random Advection," Phys. Review E 49, 474.

A. R. Kerstein and P. A. McMurtry, "Low-Wave-Number Statistics of Randomly Advected Passive Scalars," Phys. Review E 50, 2057.

A. R. Kerstein and W. T. Ashurst, "Passage Rates of Propagating Interfaces in Randomly Advected Media and Heterogeneous Media," Phys. Rev. E 50, 1100. 
A. R. Kerstein, M. A. Cremer, and P. A. McMurtry, "Scaling Properties of Differential Molecular Diffusion Effects in Turbulence," Phys. Fluids, in press.

B. Kohler, V. V. Yakovlev, K. R. Wilson, J. Squier, K. W. DeLong, and R. Trebino, "Phase and Intensity Characterization of Femtosecond Pulses from a Chirped-Pulse Amplifier by Frequency-Resolved Optical Gating," Opt. Lett., in press.

B. Kohler, V. V. Yakovlev, K. R. Wilson, K. W. DeLong, R. Trebino, and J. Squier, "Characterization of Amplified Femtosecond Pulses for Quantum Control," in Generation, Amplification, and Measurement of Ultrashort Pulses, eds. R. Trebino and I. A. Walmsley, (SPIE Press, Bellingham, 1994), 360.

B. Kohler, V. Y. Yakovlev, K. R. Wilson, J. Squier, K. W. DeLong and R. Trebino, "Intensity and Phase Measurements of Femtosecond Pulses from a Chirped Pulse Amplifier," Ultrafast Phenomena IX, ed. W. Knox, (Springer-Verlag, 1994).

B. Kohler, V. V. Yakovlev, K. R. Wilson, J. Squier, K. W. DeLong, and R. Trebino, "Phase and Intensity Characterization of Femtosecond Pulses from a Chirped Pulse Amplifier by Frequency-Resolved Optical Gating," Opt. Lett., in press.

M. L. Koszykowski, R. C. Armstrong, J.-Y Chen, and N. J. Brown, "Turbulent Jet Flame Modeling with Comprehensive Chemical Kinetics for NO Prediction," J. Comp. Phys., in press.

T. J. Kulp, "Laboratory Measurements of New Water Vapor Absorption Parameters and the Water Vapor Continuum in the 8- to $14 \mathrm{~mm}$ Atmospheric Window," Eighth Conference on Atmospheric Radiation, (Nashville, TN, January 23-28, 1994).

T. J. Kulp, "Long-Pathlength Infrared Absorption Measurements in the 8- to $14 \mathrm{~mm}$ Atmospheric Window: Self-Broadening Coefficient Data," Proceedings of Fourth Atmospheric Radiation Measurement (ARM) Science Team Meeting, (Charleston, SC, March 1994).

T. J. Kulp, L. Thorne, and P. J. Hargis, "First-Step Modeling: Solid-Effluent Signatures," Proceedings of the Caliope Program Interim Technical Review, (Livermore, CA, April 26-28, 1994) Vol. I, 96-106.

T. J. Kulp, "Measurement of Porous Medium Velocity Fields and Their Volumetic Averaging Characteristics Using Particle Tracking Velocity," Chem. Eng. Sci., in press.

T. J. Kulp, "Experimental Analysis of Pore-Scale Flow and Transport in Porous Media," Water Resources Research, in press.

T. J. Kulp, R. Kennedy, M. DeLong, D. Garvis, and J. Stahovec, "Development of an Imaging Laser Radar for Long-Range Gas Plume Visualization," Proceedings of the Seventeenth International Laser Radar Conference, (Sendai, Japan, July 25-29, 1994), 42-45.

T. J. Kulp, "Overview of Backscatter Absorption Gas Imaging," Interdisciplinary Laser Science Conference, (Dallas, TX, October 2-7, 1994).

T. J. Kulp and R. Kennedy, "Overview of Recent Developments in Gas Plume Imaging," Optical Sensing for Environmental and Process Monitoring, (McLean, VA, November 7-10, 1994).

C. L. Ladera, K. W. DeLong, D. N. Fittinghoff, and R. Trebino, "Direct Ultrashort-Pulse Intensity and Phase Retrieval Using Frequency-Resolved Optical Gating and Computational Neural Network," Opt. Lett., in press. 


\section{Publications}

A. M. Levine, E. Ozizmir, R. Trebino, C. C. Hayden, A. M. Johnson, and K. L. Tokuda, "InducedGrating Autocorrelation of Ultrashort Pulses in a Slowly Responding Medium," J. Opt. Soc. Amer. B 11, 1609.

S. Mahalingam, J. H. Chen, and L. Vervisch, "Finite-Rate Chemistry and Transient Effects in Simulations of Turbulent Non-premixed Flames," Combust. Flame, in press.

S. B. Margolis, "Resonant Mode Interactions and the Bifurcation of Combustion-Driven Acoustic Oscillations in Resonance Tubes," SIAM J. Appl. Math. 54.

S. B. Margolis, "Effects of Two-Phase Flow on the Deflagration of Porous Energetic Materials," Thirtythird AIAA Aerospace Sciences Meeting, (Reno, NV, January 9-12, 1995).

S. B. Margolis, "The Nonlinear Dynamics of Intrinsic Acoustic Oscillations in a Model Pulse Combustor," Combust. Flame 99, 311.

S. B. Margolis and F. A. Williams, "Influence of Porosity and Two-Phase Flow on Diffusional/ Thermal Instability of a Deflagrating Energetic Material," Combust. Sci. Technol., in press.

S. B. Margolis and F. A. Williams, "Influence of Porosity and Two-Phase Flow on Diffusional/ Thermal Instability of a Deflagrating Energetic Material," Fifteenth International Colloquium on the Dynamics of Explosions and Reactive Systems, (University of Colorado, Boulder, CO, July 30-August 4, 1995).

S. B. Margolis, "Effects of Two-Phase Flow on the Deflagration of Porous Energetic Materials," Invited paper, J. Prop. Power, in press.

S. B. Margolis, "The Nonlinear Dynamics of Intrinsic Acoustic Oscillations in Combustion-Driven Systems," in Mathematical Modeling in Combustion (Lecture Notes in Physics), in press.

K. D. Marx, C. F. Edwards and W. K. Chin, "Limitations of the Ideal Phase-Doppler System: Extension to Spatially and Temporally Inhomogeneous Particle Distributions with an Application to Diesel Sprays," Atomization \& Sprays, 4, 1.

A. R. Masri, R. W. Dibble, and R. S. Barlow, "The Structure of Turbulent Nonpremixed Flames Revealed by Raman-Rayleigh-LIF Measurements," Prog. Energy \& Combust. Sci., in press.

A. R. Masri, B. B. Dally, R. S. Barlow, and C. D. Carter, "The Structure of Laminar Diffusion Flames Inhibited with $\mathrm{CF}_{3} \mathrm{Br}$," Combust. Sci. Technol., in press.

T. S. McKechnie, T. J. Kulp, and P. J. Hargis, "Effect of Laser Speckle on Ultraviolet Laser Remote Sensing Measurements," Proceedings of the Caliope Program Interim Technical Review, (Livermore, CA, April 26-28, 1994) Vol. II, 38-46.

E. Meeks, A. Ting, J. F. Grcar, and R. J. Kee, "Flame-Centered Grid Transformation for Numerical Simulation of Strained Flames," Combust. Flame 96, 179.

G. Meijer, M. Verslius, and D. W. Chandler, "Degenerate Four-Wave Mixing with a Tunable Excimer Laser," Appl. Opt. 33, 3289.

P. C. Miles, R. M. Green, and P. O. Witze, "Comparison of In-Cylinder Scavenging Flows in a TwoStroke Cycle Engine under Motored and Fired Conditions," Proceedings of Seventh International Symposium on Applications of Laser Techniques to Fluid Mechanics, (Lisbon, Portugal, July 1994). 
P. C. Miles and P. O. Witze, "Fringe Field Quantification in an LDV Probe Volume by use of a Magnified Image," Exp. Fluids 16, 330.

J. A. Miller, J. V. Volponi, and J.-F. Pauwels, "The Effect of Allene Addition on the Structure of a Rich $\mathrm{C}_{2} \mathrm{H}_{2} / \mathrm{O}_{2} /$ Ar Flame," Combust. Flame, in press.

L. Minier and R. Behrens, Jr., "Thermal Decomposition Study of 2,4-Dinitroimidazole (24DNI) Using Simultaneous Thermogravimetric Modulated Beam Mass Spectrometry (STMBMS)," American Defense Preparedness Association: Energetic Materials Technology Symposium, (Orlando, FL, March 2124, 1994).

L. M. Minier, R. Behrens, Jr., "Thermal Decomposition Mechanisms of Bis (2-fluoro-2, 2-dinitroethyl) Formal (FEFO) and Bis (2-Fluoro-2, 2-Dinitro) Difluoroformal (DFF) from STMBMS Measurements," Propel. Explos. Phyrotech., in press.

H. N. Najm and A. F. Gohniem, "Numerical Simulation of Coupling Between Vorticity and Pressure Oscillations in Combustion Instability," AIAA J. Propulsion and Power 10, 769.

Q. V. Nguyen, R. W. Dibble, C. D. Carger, G. J. Feichtner, and R. S. Barlow, "Laser Measurements of Temperature, the Major Species, $\mathrm{OH}$, and NO in a Methane-Air Bunsen Flame," Combust. Flame, in press.

T. H. Osterheld, M. D. Allendorf, and C. F. Melius, "Unimolecular Decomposition of Methyltrichlorosilane: RRKM Calculations," J. Phys. Chem. 98, 6995.

T. H. Osterheld and M. D. Allendorf, "The Decomposition of Methyltrichlorosilane in Hydrogen and Helium," Materials Research Society Meeting, (Boston, MA, November 28-December 2, 1994).

P. H. Paul, J. A. Gray, J. L. Durant, Jr. and J. W. Thoman, Jr., "Collisional Quenching Corrections for Laser-Induced Fluorescence Measurements of NO A ${ }^{2} \mathrm{~S}^{+}, "$ AAIA J. 32, 1670.

P. H. Paul, R. L. Farrow, and P. M. Danehy, "Gas-Phase Thermal-Grating Contributions to Four-Wave Mixing," J. Opt. Soc. Am. B 12 .

P. H. Paul and J. E. Dec, "Imaging of Reaction Zones in Hydrocarbon-Air Flames using Planar LaserInduced Fluorescence of $\mathrm{CH}$," Opt. Lett. 19, 998.

P. H. Paul, C. D. Carter, J. A. Gray, J. L. Durant, Jr., and M. R. Furlanetto, "Correlations for the $\mathrm{OH}$ $\mathrm{A}^{2} \mathrm{~S}^{+}\left(\mathrm{v}^{\prime}=0\right)$ Electronic Quenching Cross-Section," Sandia Report, SAND94-8244.

P. H. Paul, J. A. Gray, J. L. Durant, Jr., and M. R. Furlanetto, "Collisional Electronic Quenching of OH $\mathrm{A}^{2} \mathrm{~S}^{+}\left(\mathrm{v}^{\prime}=0\right)$ Measured at High Temperature in a Shock Tube," J. Chem. Phys., in press.

P. H. Paul, "Vibrational Energy Transfer and Quenching of $\mathrm{OH} \mathrm{A}{ }^{2} \mathrm{~S}\left(v^{\prime}=1\right)$ Measured at High Temperature in a Shock Tube," J. Phys. Chem., in press.

P. H. Paul, "A Model for Temperature-Dependent Collisional Quenching of $\mathrm{OH} \mathrm{A}^{2} \mathrm{~S}^{+}$, " J. Spectr. Quant. Rad. Trans. 51, 511.

J.-F. Pauwels, J. V. Volponi, and J. A. Miller, "Allene Oxidation in a Low-Pressure $\mathrm{H}_{2} / \mathrm{O}_{2} / \mathrm{Ar}-\mathrm{C}_{3} \mathrm{H}_{4}$ Flame," Combust. Sci. Technol., in press.

L. A. Rahn, Degenerate Four-Wave Mixing Line Shapes of $\mathrm{OH}$ at High Pump Intensities," Appl. Opt., in press. 


\section{Publications}

L. A. Rahn and M. S. Brown, "Polarization Properties of Degenerate Four-Wave Mixing in Flame OH," Opt. Lett. 19, 1249.

G. H. Richards, J. N. Harb, and L. L. Baxter, "Investigation of Mechanisms for the Formation of Fly Ash Deposits from Two Low-Sulfur Western Coals, Energy E Fuels, in press.

E. A. Rohlfing, "Spectroscopic Studies of Large-Amplitude Motion in Small Clusters," Advances in Metal and Semiconductor Clusters, Vol. III, Cluster Spectroscopy, ed. M. D. Duncan, JAI Press, Inc., in press.

S. C. Ross, T. J. Butenhoff, E. A. Rohlfing and C. M. Rohlfing, "SiC2: A Molecular Pinwheel," J. Chem. Phys. 100, 4110

R. W. Schefer, M. Namazian, and J. Kelly, "Stabilization of Lifted Turbulent-Jet Flames," Combust. Flame 99, 75.

G. O. Sitz and R. L. Farrow, "Preparation and Decay of Alignment in $\mathrm{N}_{2}(\mathrm{v}=1), "$ J. Chem. Phys. 101, 4682.

L. L. Smith, R. W. Dibble, L. Talbot, R. S. Barlow, and C. D. Carter, "Laser Raman Scattering Measurements of Differential Molecular Diffusion in Nonreacting Turbulent Jets of $\mathrm{H}_{2} / \mathrm{CO}_{2}$ Mixing with Air," Phys. Fluids, in press.

R. Sondergaard, J. H. Chen, J. Soria, and B. J. Cantwell, "Topology of Fine Scale Motions in Turbulent Shear Flows," Phys. Fluids, in press.

R. R. Steeper and S. F. Rice, "Optical Monitoring of the Oxidation of Methane in Supercritical Water," Western States Section/The Combustion Institute Spring Meeting, (Davis, CA, March 21, 1994).

R. R. Steeper and S. F. Rice, "Optical Monitoring of the Oxidation of Methane in Supercritical Water," Twelfth International Conference on the Properties of Water and Steam, (Orlando, FL, September 11-16, 1994).

G. Taft, A. Rundquist. M. M. Murnane, H. C. Kapteyn, K. W. DeLong, R. Trebino, and I. P. Christov, "Ultrashort Optical Waveform Measurement Using Frequency-Resolved Optical Gating," Opt. Lett., in press.

J. D. Tobiason, J. R. Dunlop, and E. A. Rohlfing, "Dispersed Fluorescence Spectroscopy of Jet-Cooled DCO," Chem. Phys. Lett., in press.

R. Trebino and I. A. Walmsley, ed., "Generation, Amplification, and Measurement of Ultrashort Laser Pulses," Vol. 2116 (Society of Photo-Optical Instrumentation Engineers, Bellingham, WA, 1994).

J. V. Volponi and M. C. Branch, "Structure of $\mathrm{H}_{2}-\mathrm{NO}_{2}$-Argon and $\mathrm{H}_{2}-\mathrm{O}_{2}$-Argon Laminar Premixed Flames," Combust. Sci. Technol., in press.

S. R. Vosen, J. O. Keller, M. D. Allendorf, T. T. Bramlette, and R. Kaminsky, "A Pulsating Combustionbased Volatile Organic Compound Destruction Technique," Combust. Sci. Technol., in press.

S. R. Vosen, R. W. Carling, R. E. Rychnovsky, S. K. Griffiths and R. F. Renzi, "The Effect of Elastomeric Acoustic Liners on High-Pressure Liquid Propellant Combustion Oscillations," Propell., Explos. \& Pyrotech., in press. 
J. Warnatz, M. D. Allendorf, R.J. Kee, and M. E. Coltrin, "A Model of Elementary Chemistry and Fluid Mechanics in the Combustion of Hydrogen on Platinum Surfaces," Combust. Flame 96, 393.

S. Williams, R. N. Zare, and L. A. Rahn, "Reduction of Degenerate Four-Wave Mixing Spectra to Relative Populations I: Weak-Field Limit," J. Chem. Phys. 101, 1093.

S. Williams, R. N. Zare, and L. A. Rahn, "Reduction of Degenerate Four-Wave Mixing Spectra to Relative Populations II: Strong-Field Limit," J. Chem. Phys. 101, 1072.

S. Williams, L. A. Rahn, P. H. Paul, J. W. Forsman, and R. N. Zare, "Laser-Induced Thermal Grating Effects in Flames," Opt. Lett. 19, 1681.

S. Williams, J. D. Tobiason, J. R. Dunlop, and E. A. Rohlfing, "Stimulated Emission Pumping Spectroscopy via Two-Color Resonant Four-Wave Mixing," J. Chem. Phys., in press.

P. O. Witze, "Interpretation of Head-Gasket Ionization-Probe Measurements using a Twa-Zone Spherical Flame Model," Third International Symposium on Diagnostics and Modeling of Combustion in Internal Combustion Engines, (JSME, Yokohama, 1994), 453-458.

M. J. Wornat, B. G. Porter, and N. Y. C. Yang, "Single droplet Combustion of Biomass Pyrolysis Oils," J. Energy \& Fuels, in press.

D. L. Yang, M. L. Koszykowski, and J. L. Durant, "The Reaction of $\mathrm{NH}_{2}\left(X^{2} \mathrm{~B}_{1}\right)$ with $O\left(X^{3} P\right)$ : A Theoretical Study Employing Gaussian 2 Theory," J. Chem. Phys. 101, 1361.

N.Y.C. Yang and L. L. Baxter, "Instrument and Sample Preparation Considerations for ComputerControlled Scanning Electron Microscopy (CCSEM) Analyses," Conference on Inorganic Transformations and Ash Deposition During Combustion (Palm Coast, FL) , 191-206. 


\section{Index of Authors}

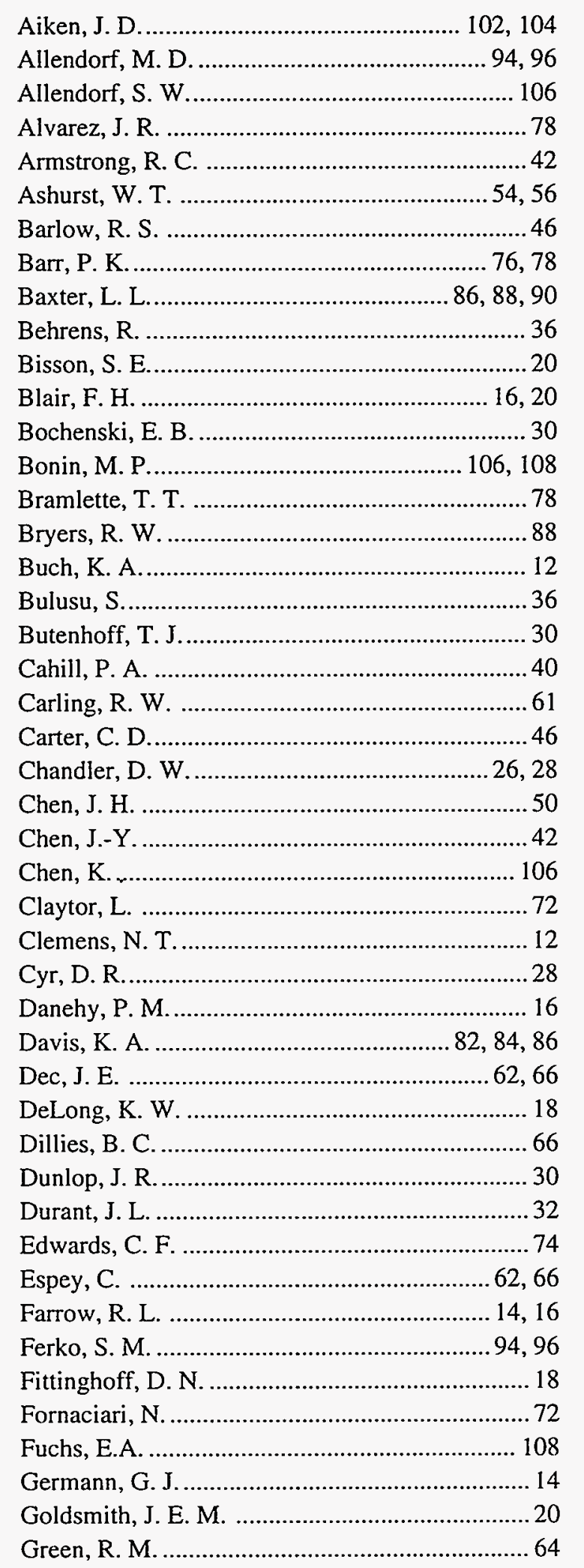

Gutzler, M. A. ............................................ 28, 32

Hanush, R. G. .......................................... 100, 102

Harb, J. N. ...................................................... 90

Hardesty, D. R. ......................................... 81, 93

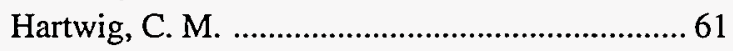

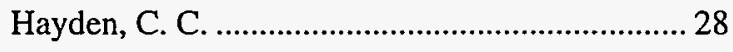

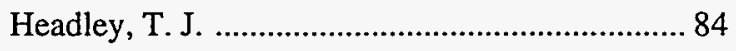

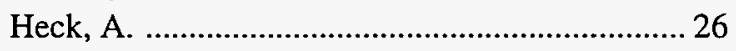

Henderson, C. C. ............................................... 40

Huey, S. P.......................................................... 86

Hurt, R. H. ......................................... 82, 84, 108

Jaska, M....................................................... 26

Jenkins, B. M. .......................................... 86, 88

Jennings, R. T.................................................... 34

Johnsen, H. A. ............................................... 106

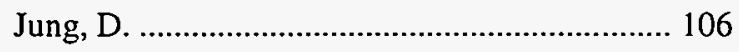

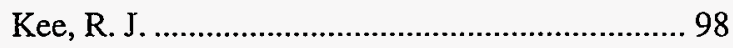

Keller, J. O. ........................................... 61, 76, 78

Kelly, J. ........................................................... 48

Kennedy, R .................................................... 22

Kent, F. W. ............................................. 106, 108

Kerstein, A. R................................................... 54

Kezerle, J. A. ……………………………...... 76

Kitsopoulos, T. .................................................... 26

Koszykowski, M. L. .............................................. 42

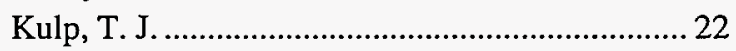

Ladera, C. L. .................................................... 18

LaJeunesse, C. A. ............................................. 102

Larson, R. S. ...................................................... 98

Lucht, R. P..................................................... 16

Mahalingam, S. ................................................. 50

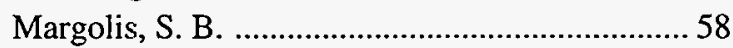

Marx, K. D. ........................................... 66, 74

McKay, R. ......................................................... 26

McLean, W. J. ............................................... 2

McMichael, J. C. .................................................... 4

Meeks, E. ........................................................ 98

Melius, C. F. ............................................. 94

Miles, P. C. .................................................. 64, 68

Miles, T. R.

Miles, T. R., Jr. ................................................... 88

Miller, J. A. ....................................................... 38

Minier, L. M. ................................................... 36

Mitchell, M. G.................................................. 20

Naber, J. D............................................................. 68

Najm, H. N. ....................................................... 52 
Namazian, M...................................................48

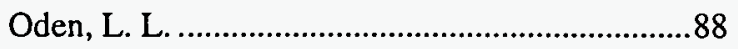

Osterheld, T. H. ..........................................94, 96

Ottesen, D. K.............................................90, 106

Paul, P. H.

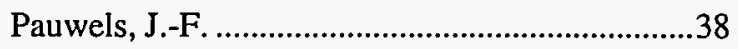

Porter, B. G. ........................................................... 86

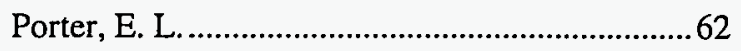

Prast, T. L. ..........................................................46

Puckett, D. M.....................................................36

Rahn, L. A..............................................11, 45

Rakestraw, D. J. ...............................................14

Rice, S. F..................................100, 102, 104

Richards, G. H....................................................90

Rohlfing, C. M. ....................................................40

Rohlfing, E. A. ........................................................

Ross, J. ........................................................ 82,86

Paul, P. H. ................................................ 12, 16

Salmi, A. J...................................86, 88, 90, 108

Sanford, R. .................................................... 72

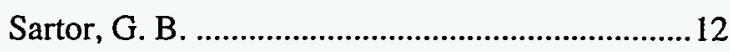

Schefer, R. W......................................................

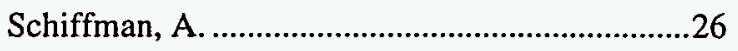

Schlippa, G. C.

Shon, J. W. $86,88,90$

Siebers, D. L.

Steeper, R. R.

Stelts, P. D.

Taatjes, C. A.

Thiesemann, H. S.

Trebino, R.

Tobiason, J. D.

Tully, F. P.

Vervisch, L.

Volponi, J. V.

Vosen, S. R.

Wang, J.

Williams, F. A.

Williams, S.

Winter, F.

Witze, P. O.

Wolf, $M$.

Wornat, M. J.

Yang, D. L.

Yang, N. Y. C.

Zare, R. N.

\section{.98}

68

100,104

106, 108

.34

32 18 30

25

50

38

98

106

.58

30

.86

64,70

.32

84,86

.32

84

26 